\title{
The impact of the 'Western Diet' on emotional, social and cognitive behaviours as revealed by a study on conventional and serotonin transporter-deficient mice
}

Citation for published version (APA):

Veniaminova, E. (2020). The impact of the 'Western Diet' on emotional, social and cognitive behaviours as revealed by a study on conventional and serotonin transporter-deficient mice. [Doctoral Thesis, Maastricht University]. Maastricht University. https://doi.org/10.26481/dis.20200305ev

Document status and date:

Published: 01/01/2020

DOI:

$10.26481 /$ dis.20200305ev

Document Version:

Publisher's PDF, also known as Version of record

Please check the document version of this publication:

- A submitted manuscript is the version of the article upon submission and before peer-review. There can be important differences between the submitted version and the official published version of record.

People interested in the research are advised to contact the author for the final version of the publication, or visit the DOI to the publisher's website.

- The final author version and the galley proof are versions of the publication after peer review.

- The final published version features the final layout of the paper including the volume, issue and page numbers.

Link to publication

\footnotetext{
General rights rights.

- You may freely distribute the URL identifying the publication in the public portal. please follow below link for the End User Agreement:

www.umlib.nl/taverne-license

Take down policy

If you believe that this document breaches copyright please contact us at:

repository@maastrichtuniversity.nl

providing details and we will investigate your claim.
}

Copyright and moral rights for the publications made accessible in the public portal are retained by the authors and/or other copyright owners and it is a condition of accessing publications that users recognise and abide by the legal requirements associated with these

- Users may download and print one copy of any publication from the public portal for the purpose of private study or research.

- You may not further distribute the material or use it for any profit-making activity or commercial gain

If the publication is distributed under the terms of Article $25 \mathrm{fa}$ of the Dutch Copyright Act, indicated by the "Taverne" license above, 
The impact of the 'Western diet' on emotional, social and cognitive behaviours

as revealed by a study on conventional and serotonin transporter-deficient mice

Ekaterina Veniaminova 
The impact of the 'Western diet' on emotional, social and cognitive behaviours as revealed by a study on conventional and serotonin transporter-deficient mice

Ekaterina Veniaminova 
(C) E. Veniaminova, Maastricht 2020.

The impact of the 'Western diet' on emotional, social and cognitive behaviours as revealed by a study on conventional and serotonin transporter-deficient mice

Cover: Natalia Nesterova, "A feast in time of plague", 1993, private collection

All rights reserved. No parts of this book may be reproduced or transmitted in any form or by any means, without prior permission in writing by the author, or when appropriate, by the publishers of the publication. 


\section{The impact of the 'Western diet' on emotional, social and cognitive behaviours as revealed by a study on conventional and serotonin transporter-deficient mice}

\section{DISSERTATION}

to obtain the degree of Doctor at the Maastricht University, on the authority of the Rector Magnificus,

Prof.dr. Rianne M. Letschert

in accordance with the decision of the Board of Deans, to be defended in public on Thursday, March $5^{\text {th }} 2020$, at 12:00 hours

by

Ekaterina Veniaminova 


\section{Promotor:}

Prof. K.-P. Lesch

\section{Co-promotors:}

Prof. T. Strekalova

Prof. D.C. Anthony, University of Oxford

\section{Assessment Committee:}

Prof. Boris W. Kramer (Chairman)

Prof. Wilfred T.V. Germeraad

Dr. Ehsan S.E. Pishva

Prof. Lee Wei Lim, University of Hong Kong

Dr. Fay Probert, University of Oxford 


\section{Contents}

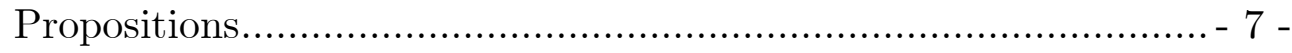

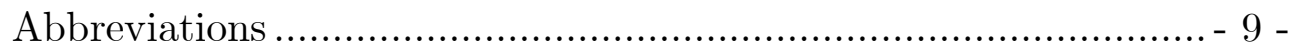

Chapter 1. General introduction................................................. - 14 -

1.1 'Western diet' as an environmental factor in psychiatric

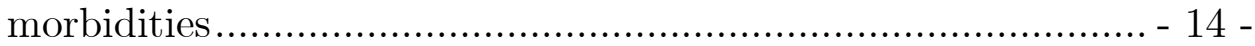

1.2 Pathophysiological mechanisms of negative effects of Western diet on the brain ................................................................ - 17 -

1.3 Peripheral effects of Western diet relevant to neuropsychiatric

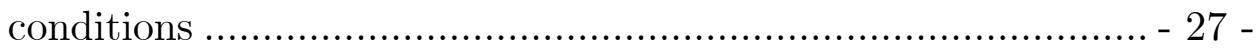

1.4 Role of decreased function of the serotonin transporter (SERT) in metabolic and mental disorders .......................................... - 33 -

1.5 Studying the effects of Western diet on brain functions using animal models..................................................................... 35 -

Hypothesis and aim ............................................................. 39 -

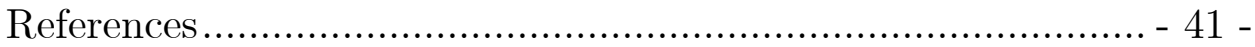

Chapter 2. Autism-like behaviours and memory deficits result from a Western diet in mice ........................................................... - 62 -

Chapter 3. Prefrontal cortex inflammation and liver pathologies accompany cognitive and motor deficits following Western diet consumption in non-obese female mice ........................................ 108 -

Chapter 4. Metabolic, molecular and behavioural effects of Western diet in serotonin transporter-deficient mice: rescue by heterozygosity? $-170-$

Chapter 5. General discussion $-228-$

5.1 Environmental factors aggravating autism spectrum disorder (ASD) and attention-deficit/hyperactivity disorder (ADHD) symptoms: focus on diet $228-$ 
5.2 Toll-like receptor 4 (Tlr4) and microglia activation in ASD, ADHD and depression........................................... 231 -

5.3 Oxidative stress and mitochondrial dysfunction in ASD, ADHD and depression......................................................... 237 -

5.4 Interplay between peroxisome proliferator-activated receptors (PPARs), Tlr4, serotonin transporter and insulin receptors activity. - 246 -

5.5 Direct and peripheral inflammation-mediated effects of Western diet components on neuroinflammation and brain mitochondrial activity - 252 -

5.6 The role of Sert deficiency in the interaction between genetic predisposition to psychiatric disorders and diet ................... 258 -

5.7 Limitations of animal models of Western diet and Sert deficiency when studying human pathologies .................................. 264 -

5.8 Future perspectives .............................................. 269 -

References ................................................................. 270 -

Summary …................................................................ 295 -

Samenvatting .............................................................. 298 -

Valorization ............................................................ 301 -

Acknowledgments ................................................... 307 -

About the author ............................................................ 312 - 


\section{Propositions}

1. The Western diet suppresses social interactions, impairs cognition, induces impulsive and depressive-like behaviours, which are, together, all reminiscent of symptoms of autism spectrum disorder (ASD) and attention-deficit/hyperactivity disorder (ADHD) (this thesis);

2. The Western diet-induced abnormalities are associated with signs of oxidative stress, microglial activation, decreased gene expression of serotonin transporter in the prefrontal cortex as well as metabolic impairments (this thesis);

3. Complete genetic serotonin transporter inactivation exacerbates metabolic, molecular, and the behavioural consequences of the Western diet in aging mice (this thesis);

4. Mice with partial genetic serotonin transporter deficiency display a 'rescued' phenotype in dietary-induced abnormalities in glucose tolerance, neuroinflammation and hippocampus-dependent behaviour (this thesis);

5. Invention of a specific diet might provide relief for patients with co-occurring metabolic and neuropsychiatric disorders, which will decrease costs associated with disability and health management (this thesis, valorization); 
6. Diet might impact on the pathogenesis of other neuropsychiatric disorders not considered in this thesis (for example, psychosis);

7. Other diets such as Mediterranean diet is likely to be protective in adulthood in cases of $\mathrm{ADHD} / \mathrm{ASD}$;

8. Gut microbiota has an important role in the interaction between diet and brain dysfunction;

9. "In food excellent medicine can be found, in food bad medicine can be found; good and bad are relative" (Hippocrates, "De Alimento", 5th-4th century $B C$ );

10. "Dilettante gardeners love the spring and summer; real gardeners also love the winter" (Anne Scott-James, "Down to Earth", 1971);

11. "Today's philosophy questions are tomorrow's precision science experiments" (Jorge Cham and Daniel Whiteson, "We Have No Idea: A Guide to the Unknown Universe", 2017). 


\section{Abbreviations}

$3-\mathrm{NT}$

5-HIAA

5-HIAA

5-HT

8-oxo-dG

8-oxoG

ADHD

Akt

AP-1

ASD

ATP

BBB

$\mathrm{BH} 4$

CAT

CNS

COX

CRP

CSF

CVO

DHA

DNA

EC

EPA 3-nitrotyrosine

5-hydroxyindoleacetic acid

5-hydroxyindoleacetic acid

Serotonin

8-oxo-deoxyguanosine

8-oxo-7,8-dihydroguanine

Attention-deficit/hyperactivity disorder

Protein kinase B

Activator protein-1

Autism spectrum disorder

Adenosine triphosphate

Blood brain barrier

Tetrahydrobiopterin

Catalase

Central nervous system

Cyclooxygenase

C-reactive protein

Cerebrospinal fluid

Circumventricular organs

Docosahexaenoic acid

Deoxyribonucleic acid

Enterochromaffin cell

Eicosapentaenoic acid 
FFA

FK506

GLUT

GSH

GSSG

GST

HDL

HDL

HPA

HPLC

IDO

IFG

IFN

IGT

IKK

IL

IL-1Ra

IR

IRS

$\mathrm{I} \chi \mathrm{B} \alpha$

JNK

LDL

LPS

MAPK
Free fatty acids

Protein binding protein 5

Glucose transporter

Glutathione

Glutathione disulfide

Glutathione-S-transferase

High density lipoprotein

High density lipoprotein

Hypothalamic-pituitary-adrenal axis

High performance liquid chromatography

Indoleamine 2,3 dioxygenase

Impaired fasting glucose

Interferon

Impaired glucose tolerance

$\mathrm{I} \chi \mathrm{B}$ kinase

Interleukin

Interleukin-1 receptor antagonist

Insulin receptors

Insulin receptor substrate

Nuclear factor of kappa light polypeptide gene

enhancer in B-cells inhibitor, alpha

N-terminal kinase Jun

Low density lipoprotein

Lipopolysaccharide

Mitogen-activated protein kinase 
MCP-1

MDA

MDD

mtDNA

NAFLD

$\mathrm{NASH}$

$\mathrm{NF}-x \mathrm{~B}$

$\mathrm{NO}$

NOS

OSI

$\mathrm{PDH}$

PET

PGE2

PI3K

PKA

PKC

PPAR

PPARGC

PUFA

ROS

SERT

SFA

SGLT

SNP
Monocyte chemotactic protein-1

Malondialdehyde

Major depressive disorder

Mitochondrial DNA

Non-alcoholic fatty liver disease

Non-alcoholic steatohepatitis

Nuclear factor $x \mathrm{~B}$

Nitric oxide

Nitric oxide synthase

Oxidative stress index

Pyruvate dehydrogenase

Positron emission tomography

Prostaglandin E2

Phosphoinositide 3-kinase

Protein kinase A

Protein kinase $\mathrm{C}$

Peroxisome proliferator-activated receptor

Peroxisome proliferator-activated receptor gamma coactivator

Polyunsaturated fatty acid

Reactive oxygen species

Serotonin transporter

Saturated fatty acids

Sodium-dependent glucose transporters

Single nucleotide polymorphisms 
SOD

SSRI

STAT

$\mathrm{T} 2 \mathrm{D}$

TAS

TIRAP

TLR

TNF

TOS

VMAT2

WT

$\mathrm{XO}$
Superoxide dismutase

Selective serotonin reuptake inhibitor

Signal transducer and activator of transcription

Type 2 diabetes

Total antioxidant status

TIR domain-containing adaptor protein

Toll-like receptor

Tumor necrosis factor

Total oxidant status

Vesicular monoamine transporter 2

Wild type

Xanthine oxidase 


\section{Chapter 1}

\section{General introduction}




\section{Chapter 1. General introduction}

\section{1 'Western diet' as an environmental factor in psychiatric morbidities}

The 'Western diet' is a dietary pattern that comprises a high intake of saturated fat, cholesterol, sugars, red meats, and salt, typically in the form of refined and processed foods. It is low in fresh fruits and vegetables, whole grains and seafood (Carrera-Bastos et al., 2011). Diet typical of Western societies was brought about by lifestyle changes following the 19th century Industrial Revolution, also known as the First Industrial Revolution, which introduced new methods of food processing, widespread use of refined vegetable oils and sugars and the advent of the 'junk-food' and 'fast-food' industry in the 1950s (Carrera-Bastos et al., 2011; Cordain et al., 2005). Western-style food consumption has been increasing consistently since then, especially in Western-culture countries (Fig. 1.1). The BBC Good Food Nation Survey 2016 found that people in the UK ate fast food on average two days per week, with young adults eating it 4.5 times a week. In the USA, on any given day, $34 \%$ of children consume fast-food and in general $44 \%$ eat fast-food once a week and 20\% twice a week (Pew Research Center). Due to a growing economy, higher household income and increased globalization, the rates of fast-food consumption are also increasing in China (Wang et al., 2016), Japan (USDA Foreign Agricultural Service), India (Euromonitor International, 2017), and 
Middle Eastern countries (Sarant et al., 2013, Naeem et al., 2012). Here, Western-style meals, snacks and junk food are at odds with the traditional food culture.

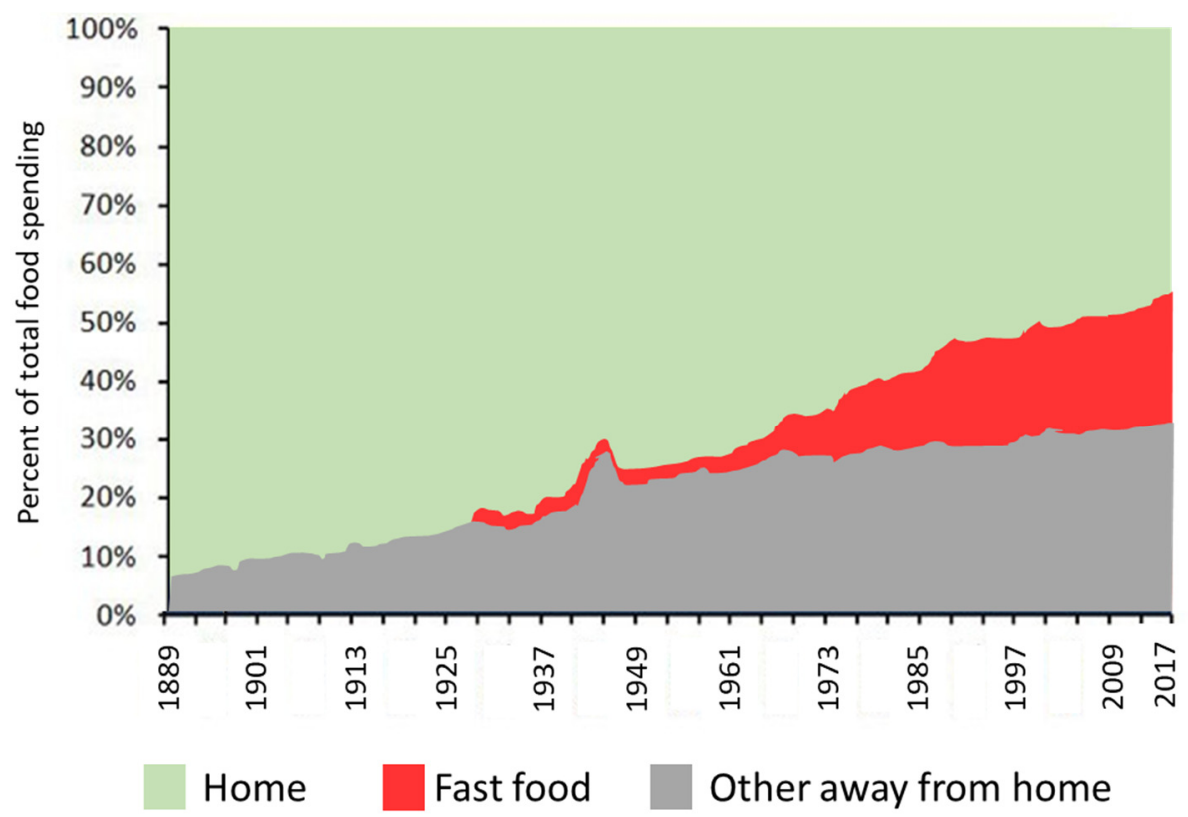

Figure 1.1. Eating habits dynamics in the USA from 1889 till 2017. Fast food expenditures were not tracked before 1929. In 1930-1960 fast food was not a significant expenditure, after which it rapidly gained in popularity. Today, fast food accounts for nearly $20 \%$ of total food expenditures. In 2017, less than half of food was consumed at home, the rest was consumed in either full-service or fast-food restaurants. Adapted from Guyenet, 2011 and USDA report, 2018.

The 21st century is also characterized by an increasing prevalence of psychiatric disorders, with challenges both for the individuals and wider society (Fuhrer and Keyes, 2019). Although the determinants of mental health are complex, there is emerging evidence for nutrition as an important factor in the prevalence of mental disorders (Sarris et al., 
2015). There is a growing amount of observational data regarding the association between dietary factors, such as Western diet consumption, and mental health. A higher risk of depression has been found in an adult populations with higher consumption of processed foods (Akbaraly et al., 2009) and fast food (Sánchez-Villegas et al., 2012). In women, Western diet adherence was found to be associated with major depression, dysthymia and anxiety disorders (Jacka et al., 2010). A systematic review has confirmed a relation between unhealthy dietary patterns and poorer mental health in children and adolescents (O'Neil et al., 2014).

On the other hand, adherence to Mediterranean and other regional traditional diets, which are typically high in vegetables, fruit, fish, nuts, beans and whole grains, were associated with reduced risk of depression (Le Port et al., 2012; Psaltopoulou et al., 2013) and cognitive impairment (Psaltopoulou et al., 2013). Dietary interventions, such as reducing intake of sugary, fried and processed food and fast-food were shown to decrease depressive symptomatology scores, assessed using the rating scale, in patients (Jacka et al., 2017; Reynolds et al., 2014). Although stress and depression can lead to unhealthy eating, recent studies have suggested that mental stateinduced alterations in eating behaviour are not likely to explain longterm associations between depression and dietary pattern (Le Port et al., 2012). 
Poor maternal dietary patterns are considered a risk factor for neurodevelopmental disorders such as autism spectrum disorder (ASD) (Li et al., 2018; Lyall et al., 2013) and attention-deficit/hyperactivity disorder (ADHD) (Millichap and Yee, 2012). Western-type diet consumption is associated with ADHD diagnosis in adolescents (Howard et al., 2011). Here, the causal link between dietary pattern and disorder development, including the underlying mechanisms involved, remain to be elucidated.

\subsection{Pathophysiological mechanisms of negative effects of Western diet on the brain}

Inflammation presents one potential mechanism through which nutrition may affect the brain (Minihane et al., 2015). This is supported by several studies showing that a higher dietary inflammatory index, the measure providing an estimate of the inflammatory potential of a diet from individual food parameters, is associated with increased risk of psychiatric disorders, including major depression and bipolar disorder (Firth et al., 2018; Oddy et al., 2018). One meta-analysis also found that a high dietary inflammatory index is more strongly associated with depression among females than males (Oddy et al., 2018). There is an emerging body of literature suggesting that elevated peripheral inflammatory markers are associated with deficits in cognitive function (Allison and Ditor, 2014; Carrard et al., 
2016) as well as worse disease prognosis (Firth et al., 2018) among patients with psychiatric disorders.

Though the specific mechanisms underlying this association remain unclear, diet-induced low-grade inflammation, characterized by chronic activation of the immune system, is thought to have a number of detrimental effects on brain structure and function (Minihane et al., 2015). Several studies propose that alterations in the gut microbiota triggered by dietary changes may be a key initial step in the development of low-grade systemic inflammation, affecting the whole body (Sanmiguel et al., 2015). Data from animal models support this hypothesis, demonstrating that germ-free mice exhibit neither obesity nor upregulation of intestinal tumor necrosis factor $\alpha$ (TNF) level compared to conventionalized mice when fed with high fat diet (Turnbaugh et al., 2008). Comparing microbiota of obese and lean subjects demonstrated that high bacterial richness (diversity) is associated with less significant adiposity and inflammation than found in subjects with low bacterial richness (Le Chatelier et al., 2013). Shifts in gut microbiota may lead to increased levels of lipopolysaccharides (LPS) and increased intestinal permeability through Toll-like receptor (TLR)-dependent mechanisms (Yiu et al., 2017) (Fig. 1.2), thus promoting increased translocation of LPS into the circulation (Bleau et al., 2015; Ding et al., 2010). High levels of glucose were also shown to stimulate serotonin release from enterochromaffin cells (EC) in the 
gut (Kim et al., 2001; Racké et al., 1996), increasing extracellular serotonin levels. This resulted in the overstimulation of 5-HT3 receptors and subsequent reduction of tight-junction proteins, ultimately leading to enhanced intestinal permeability (Yamada et al., 2003).

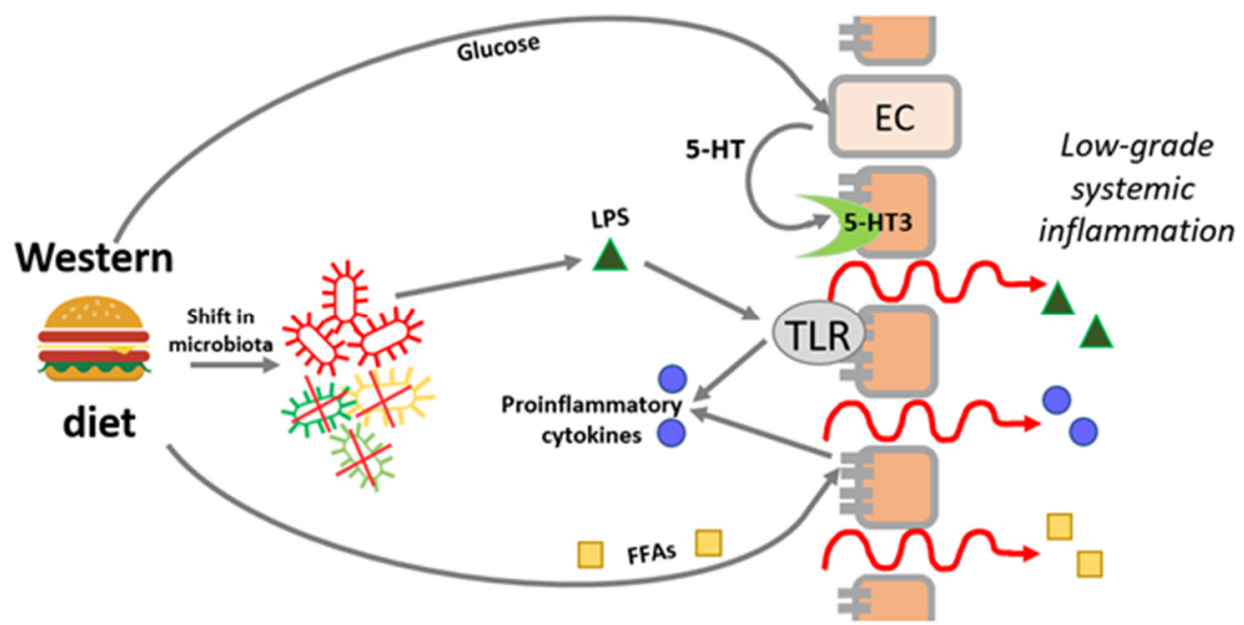

Figure 1.2. Development of systemic inflammation due to diet-induced shift in gut microbiota. Shifts in gut microbiota lead to increased levels of LPS and increased intestinal permeability due to TLR-dependent mechanisms. High levels of glucose stimulate serotonin release from the enterochromaffin cells (EC) increasing extracellular serotonin levels, which results in overstimulation of 5-HT3 receptors, reduction of tight-junction proteins and enhanced intestinal permeability. Free fatty acids (FFAs), present in high amounts in Western diet, act on intestinal cells leading to increased production of proinflammatory cytokines in the gut. Intestinal endotoxin, proinflammatory cytokines and FFAs are then delivered into the systemic circulation leading to low-grade systemic inflammation.

In addition, free fatty acids (FFAs), prevalent in the Western diet, may act directly on intestinal and endothelial cells leading to 
increased production of proinflammatory cytokines in the gut (Fujiyama et al., 2007; Konrad and Wueest, 2014; Yoshida et al., 2001). This way, intestinal LPS, proinflammatory cytokines and FFAs are delivered into the systemic circulation, leading to chronic low-grade systemic inflammation (Konrad and Wueest, 2014; Tsukumo et al., 2015).

Elevated plasma FFAs and LPS can then upregulate the expression of TLRs in circulating monocytes, leading to their activation and production of proinflammatory cytokines (Bleau et al., 2015; Kim et al., 2012). Activated inflammatory macrophages infiltrate adipose, muscle, and liver tissue, leading to a generalized peripheral inflammatory response (Bleau et al., 2015; Lumeng and Saltiel, 2011). The proinflammatory effect is mediated by at least two mechanisms, associated with the nuclear factor $\chi \mathrm{B}(\mathrm{NF}-\varkappa \mathrm{B})$ and N-terminal kinase Jun (JNK) (Bastard et al., 2006) (Fig. 1.3). The NF-xB pathway responds to diverse stimuli, including ligands of cytokine receptors, pattern-recognition receptors such as TLRs, as well as T-cell and B-cell receptors (Zhang and Sun, 2015). The primary mechanism for NF- $x$ B activation is the inducible degradation of nuclear factor of kappa light polypeptide gene enhancer in B-cells inhibitor, alpha $(\mathrm{I} \chi \mathrm{B} \alpha)$ triggered through its site-specific phosphorylation by I $\chi \mathrm{B}$ kinase (IKK) complex (Liu et al., 2017b). 


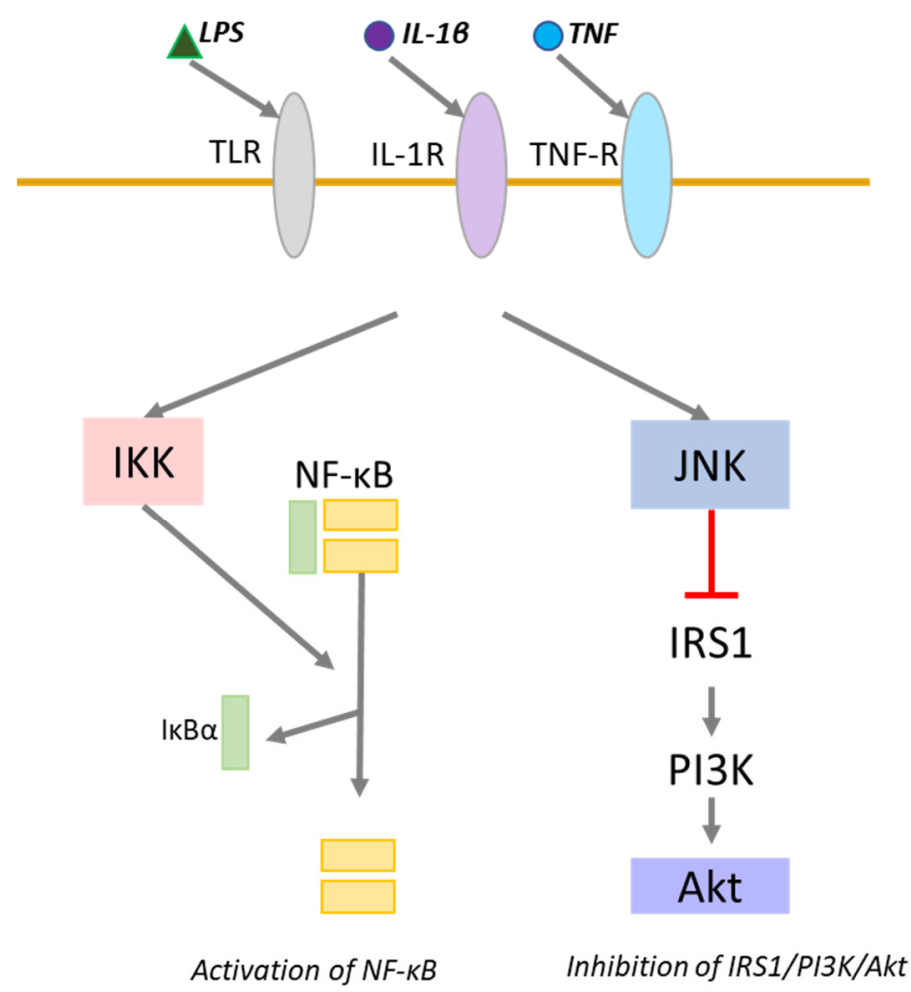

Figure 1.3. Mechanisms of NF- $\varkappa \mathrm{B}$ pathway activation and of IRS1/PI3K/Akt pathway inhibition. I $\chi \mathrm{B}$ kinase (IKK) is activated by different stimuli, including cytokines and microbial components. Upon activation, IKK phosphorylates $\mathrm{I} \chi \mathrm{B} \alpha$ and triggers its ubiquitin-dependent degradation, resulting in activation and nuclear translocation of nuclear factor $x \mathrm{~B}(\mathrm{NF}-x \mathrm{~B})$. Enhanced proinflammatory signaling also results in N-terminal kinase Jun (JNK) activation which inhibits activation of IRS1 protein and subsequently leads to the inhibition of PI3K and Akt.

In addition to mediating induction of various proinflammatory genes in innate immune cells, NF- $x \mathrm{~B}$ regulates the activation of inflammatory T cells and inflammasomes (Liu et al., 2017b). Enhanced proinflammatory signaling also results in a subsequent JNK activation, 
which leads to the inhibition of signal transduction via pathways involving protein kinase B (Akt), and associated signal transducers like insulin receptor substrate 1 (IRS1) and phosphoinositide 3-kinases (PI3K) (Aguirre et al., 2000). This pathway is a key mechanism in the regulation of glucose metabolism in insulin-sensitive peripheral tissues (Aguirre et al., 2000) and is also implicated in the general response to extracellular stress (Karin and Gallagher, 2005) and inflammation (Bogoyevitch and Kobe, 2006; Karin and Gallagher, 2005).

Ectopic lipid accumulation aggravates systemic inflammation via the recruitment of M1 macrophages and increased expression of proinflammatory mediators (Caesar et al., 2015; Lee and Lee, 2014). Leptin produced by adipocytes triggers the activation of mitogenactivated protein kinase (MAPK) p38, and signal transducer and activator of transcription (STAT) 3, which lead to increased production of proinflammatory cytokines (Tilg and Moschen, 2006). Downstream effects include the activation, proliferation and migration of monocytes. In addition, resistin expressed by adipose tissue further enhances proinflammatory cytokine production (Tilg and Moschen, 2006).

There are several pathways by which peripheral inflammation could affect the brain (Fig. 1.4). Firstly, cytokines from the blood have been shown to be able to penetrate the blood brain barrier (BBB) in regions of high BBB permeability, for example the circumventricular 
organs (CVO), and act directly on the neuronal and glial receptors, thus regulating the synthesis of cytokines in the CNS (Banks and Erickson, 2010). Cytokine influx into the CNS can also be increased in case of BBB disruption. A number of studies suggest that dietary and metabolic factors are related to disrupted BBB integrity. For example, adiposity in midlife female subjects is found to be strongly linked with reduced BBB integrity 24 years later (Gustafson et al., 2007). Increased BBB permeability has been shown in several models of the Western diet feeding in rodents (Banks et al., 2004; Kozler and Pokorný, 2003; Sparks, 2008; Wolburg and Lippoldt, 2002). Independent of BBB integrity, there are transport systems which are able to carry interleukin (IL)-1 $\alpha$, IL-1 $\beta$, IL-1Ra, IL-6 and TNF through the BBB (Banks et al., 1995). Inflammation was shown up-regulate the influx carriers responsible for TNF (Osburg et al., 2002). Circulating cytokines activate the expression of cyclooxygenase (COX), leading to increased synthesis of prostaglandins, which in turn induce cytokine production in neurons. Systemic inflammation can promote leukocyte passage across the BBB through a paracellular or transcellular route (Bechmann et al., 2007), which was demonstrated for lymphocytes (Banks et al., 2012), neutrophils (Bohatschek et al., 2001) and monocytes (Wang et al., 2008). The activated systemic immune system can also signal to the brain via the nervous pathway involving both vagal and sympathetic nerves (Banks and Erickson, 2010). 


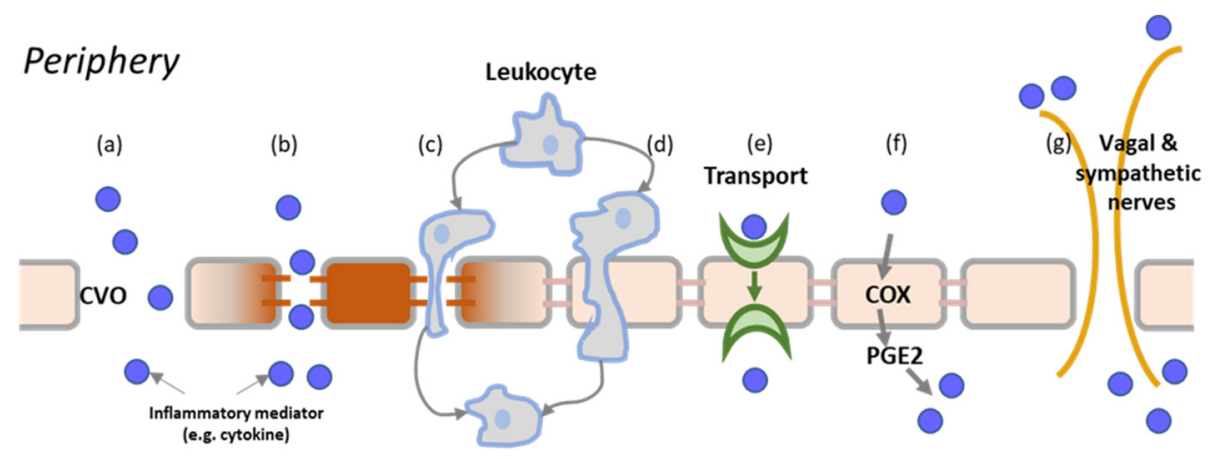

CNS

Figure 1.4. Pathways by which peripheral inflammation can affect the CNS. Inflammatory mediators (e.g. cytokines) can penetrate through the BBB in the regions of high permeability, such as (a) circumventricular organs (CVO), or (b) in case of impaired tight junction integrity. Passage of the leukocytes across the BBB may occur through (c) a paracellular or (d) transcellular route. (e) Some proinflammatory mediators can be directly transported through BBB. (f) Proinflammatory cytokines also promote cyclooxygenase (COX) expression that increases production of prostaglandin E2 (PGE2) which induces inflammatory CNS changes. (g) Vagus and sympathetic nerves are involved in signal transduction from activated immune system into the brain via the nervous pathway.

Once proinflammatory signals reach the CNS, their signaling pathways may affect neurotransmitter systems including serotonin, norepinephrine, dopamine and glutamate systems, all of which when perturbed are known to be associated with the development of mental disorders (Miller et al., 2013). First, CNS inflammation can influence the synthesis and reuptake of monoamine neurotransmitters. Inflammatory cytokines and their signaling pathways can activate the indoleamine 2,3 dioxygenase (IDO) which converts tryptophan, the primary amino acid of serotonin, into kynurenine, thus potentially 
depleting the availability of serotonin in the brain (Dantzer et al., 2008). Another mechanism by which inflammatory cytokines can influence the synthesis of monoamine neurotransmitters is through the disruption of tetrahydrobiopterin (BH4) which is an enzyme co-factor for the rate limiting enzymes for the synthesis of serotonin, dopamine and norepinephrine (Haroon et al., 2012). High levels of proinflammatory cytokines can affect dopamine packaging by decreasing expression of vesicular monoamine transporter 2 (VMAT2) (Kazumori et al., 2004). Activation of MAPK pathways was also shown to increase the expression of dopamine transporter (Morón et al., 2003). Another mechanism by which inflammatory cytokines can influence neurotransmitter function is through effects on release. Administration of interferon (IFN) a has been shown to increase the reuptake and decrease the release of dopa, the primary precursor of dopamine (Capuron et al., 2012). Inflammatory cytokines have also been shown to stimulate the release of glutamate from astrocytes and reduce astrocytic expression of glutamate transporters, potentially leading to increased glutamate excitotoxicity (Ida et al., 2008; Matute et al., 2006).

It is well-established that high intakes of fat, sugars and cholesterol can promote oxidative stress and subsequently contribute to inflammation (Aksenova et al., 2005). Despite this, the mechanisms underlying the elevation of oxidative stress in metabolic disorders are 
not fully understood. It is proposed that mitochondrial dysfunction (Yuzefovych et al., 2013) and increased fatty acid oxidation (Lee et al., 2015) contribute to these phenomena. Obese subjects are characterized by enhanced superoxic lipid oxidation in adipocytes (Furukawa et al., 2004), increased nitric oxide (NO) production and concentration of oxidative stress markers in the blood (Codoñer-Franch et al., 2011), and decreased expression of antioxidative enzymes (Le Lay et al., 2014). Reactive oxygen species (ROS) can stimulate signal transduction pathways promoting production of IL-6, TNF and monocyte chemotactic protein-1 (MCP-1) (Elmarakby and Sullivan, 2012; Park et al., 2006; Rahman et al., 2002; Sung et al., 2002). Further, oxidative stress can also induce cellular senescence, particularly adipocyte senescence, via cellular oxidation damage (MacKellar et al., 2010; Minamino et al., 2009). Adipocyte senescence leads to macrophage recruitment and increases production of proinflammatory cytokines (Lafontan, 2014; Minamino et al., 2009).

Impaired by prolonged exposure to the Western diet, peripheral insulin sensitivity and regulation may affect CNS function by influencing the efficacy of insulin signaling in the brain directly. Insulin receptors (IR) are abundantly expressed in the hypothalamus, hippocampus, cerebral cortex, cerebellum and olfactory bulb. Astrocytes express both IRA and IRB isoforms, while neurons express exclusively the IRA (Pomytkin et al., 2018). Numerous studies have 
revealed the involvement of IR in a broad spectrum of functions in the CNS, such as synapse formation and remodeling, neuronal survival and synaptic plasticity. Deficiency in IR-mediated signaling in the brain is associated with neurodevelopmental, affective and neurodegenerative disorders (Cline et al., 2012; Pomytkin et al., 2018).

Evidence suggests that levels of CNS insulin depend upon transport of peripheral insulin into the brain, as little or no insulin is produced in the CNS (Woods et al., 2003). Increased dietary fat intake was shown to reduce insulin transport into the CNS in a dog model of high-fat diet feeding (Kaiyala et al., 2000). Reduced insulin signaling in the hippocampus as well as peripheral insulin resistance were demonstrated in mice fed with the Western diet (Mielke et al., 2006). The adverse effect of excessive dietary fat and sugar intake on both peripheral and brain insulin signaling may partially explain the comorbidity of metabolic and psychiatric disorders. Together, it can be suggested that the interplay between oxidative stress and inflammation induced by the Western diet may also affect the brain, leading to the destabilization of various signaling systems including insulin signaling and neurotransmitter systems.

\subsection{Peripheral effects of Western diet relevant to neuropsychiatric conditions}

Nutrition is one of the most important factors affecting the development of metabolic disorders and their complications (Maugeri 
and Barchitta, 2019). A strong relationship can be drawn between Western-style food consumption and obesity (Drewnowski, 2007; Kant, 2004; Yang et al., 2012). Current rates of obesity have reached epidemic levels, for example, in the EU about 20-30\% of citizens are obese (Webber et al., 2014). Aside from the myriad of consequences to metabolic health, obesity is also associated with the diagnosis of mood and anxiety disorders (Bodenlos et al., 2011). Thus, major depressive disorder and obesity demonstrate a bidirectional relationship: the presence of either condition at baseline leads to a greater risk of developing the other condition (Bodenlos et al., 2011). Increased prevalence of being overweight has been noted in drug-naïve patients with bipolar disorder (Maina et al., 2008).

Children with ASD suffer from obesity and other metabolic disorders approximately two times more frequently than controls without ASD (Shedlock et al., 2016) (Fig. 1.5). ADHD has also been demonstrated to be associated with greater likelihood of being overweight (Pagoto et al., 2009) or obese (Cortese and Tessari, 2017; Fleming et al., 2005; Pagoto et al., 2009). Several studies demonstrated that obesity and associated factors, such as sleep disorders, could lead to ADHD development (Cortese et al., 2008; Gami et al., 2003; Weinberg and Brumback, 1990). Comorbid obesity and ADHD resulted in less successful weight-control treatment compared to patients with no ADHD symptoms (Altfas, 2002). In addition, paternal 
and maternal obesity were described as risk factors for both ADHD (Andersen et al., 2018; Li et al., 2016) and ASD development in children (Andersen et al., 2018; Li et al., 2016; Suren et al., 2014).

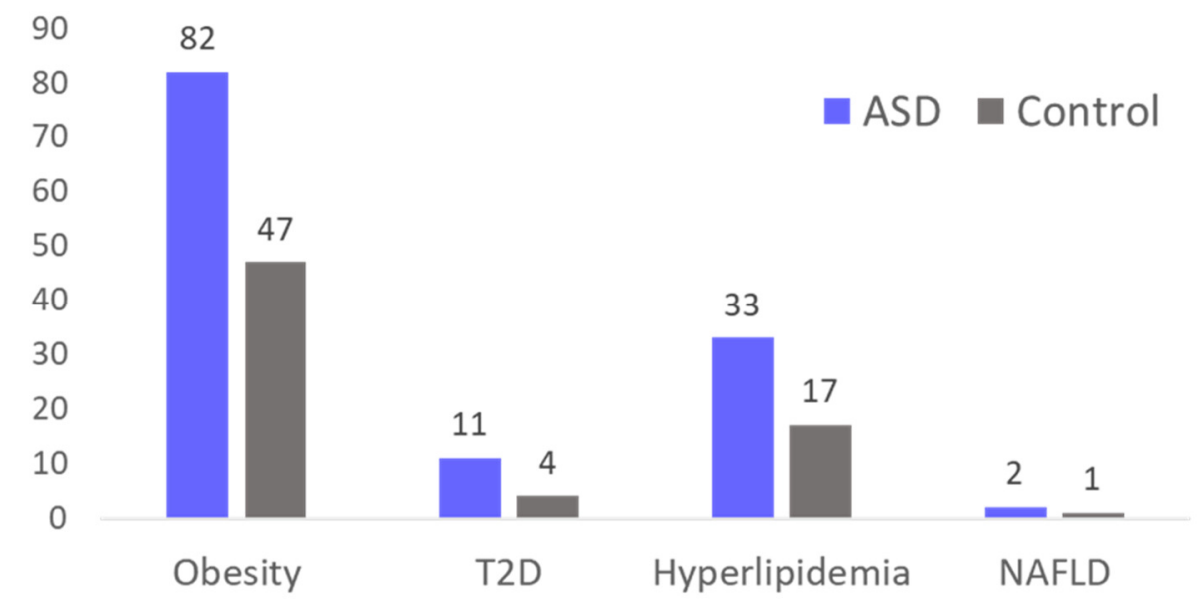

Figure 1.5. Metabolic disorders among children with ASD. The prevalence of obesity, type 2 diabetes (T2D), hyperlipidemia and non-alcoholic fatty liver disease (NAFLD) are approximately two-fold increased in children with ASD compared to controls. Adapted from Shedlock et al., 2016.

Dietary habits and sedentary lifestyle are the major factors for the rapidly rising incidence of diabetes mellitus (Sami et al., 2017). Diet higher in glycemic index and glycemic load drives glucose metabolism abnormalities such as impaired fasting glucose (IFG), impaired glucose tolerance (IGT) and can ultimately lead to a diagnosis of type 2 diabetes (T2D) (Brand-Miller et al., 2003; Salmerón, 1997; Salmeron et al., 1997). Certain foods, especially sugar-sweetened beverages (Malik et al., 2010; Schulze, 2004), as well as dietary patterns, including a Western dietary pattern (Fung et al., 2004; van 
Dam et al., 2002) are related to an increased risk of developing T2D. Dietary interventions were shown to decrease the incidence of diabetes later in life among people with IGT (Pan et al., 1997).

Disturbances in brain glucose metabolism are a feature of many psychiatric disorders, including depression (Li et al., 2015; Schweiger et al., 2008), ASD (Anil Kumar et al., 2017; Mitelman et al., 2018) and ADHD (Zametkin et al., 1990). There is an increased rate of depressive symptoms in women with impaired glucose metabolism (Adriaanse et al., 2008) and patients with T2D (Bouwman et al., 2010). Higher fasting blood glucose concentrations and IGT have been found in depressed individuals who have demonstrated suicidal behaviour (Koponen et al., 2015). Positive dietary interventions, such as adherence to a Mediterranean diet, can have a beneficial effect on the risk of depression in patients with T2D (Sánchez-Villegas et al., 2013).

Maternal gestational diabetes and $\mathrm{T} 2 \mathrm{D}$ are associated with increased odds of having a child with an ASD (Connolly et al., 2016; Li et al., 2016) or other developmental disorder (Krakowiak et al., 2012). Children and adolescents with type 1 diabetes suffer from psychiatric disorders, including ADHD, more frequently than those without diabetes (Butwicka et al., 2015). This further highlights the comorbidity between diabetes and psychiatric disorders, and the likelihood of a common pathophysiological mechanism linked to deficits 
in central insulin signaling (Kleinridders et al., 2015; Lyra E Silva et al., 2019; Pomytkin et al., 2015).

In addition to disturbances in glucose metabolism, a Western style diet can also impair metabolism of lipids (van Dam et al., 2002). This dietary pattern is positively associated with increased blood levels of total cholesterol and low density lipoprotein (LDL) cholesterol (Na et al., 2019; Sadakane et al., 2008; van Dam et al., 2003). Hypertriglyceridemia is observed in people with high intake of refined sugars (Bray and Popkin, 2014; Rippe and Angelopoulos, 2015). Total and LDL cholesterol and triglyceride levels are also higher among depressed individuals with suicidal behaviour (Koponen et al., 2015) and those with a history of anxiety disorders (Bajwa et al., 1992).

Maternal low blood level of high density lipoprotein (HDL) is associated with ADHD in offspring (Ji et al., 2017). While in some cases of ASD, deficit in cholesterol synthesis is thought to underlie the pathology (Gillberg et al., 2017), a matched case-control study found no difference in total cholesterol blood level between children with autism compared to children without autism (Kim et al., 2010). In addition, increased triglyceride level and LDL/HDL ratio were demonstrated in autistic children (Kim et al., 2010).

Dysregulated lipid metabolism is characteristic of liver diseases, such as non-alcoholic fatty liver disease (NAFLD) (Ipsen et al., 2018). NAFLD affects an estimated $25 \%$ of adults living in Western countries, 
with up to $80 \%$ of obese individuals being affected (Ruhl and Everhart, 2004). Importantly, liver steatosis, a key feature of NAFLD, can be diagnosed in the absence of obesity (Margariti et al., 2012). Diets that are high in fat and sugar, which characterize Western diets, have been shown to induce NAFLD (Hosseini et al., 2016). Several studies in patients with biopsy-proven NAFLD have linked depression with non-alcoholic steatohepatitis (NASH) (Elwing et al., 2006), hepatocyte ballooning (Youssef et al., 2013), hepatic steatosis (Weinstein et al., 2011) and NAFLD activity score (Tomeno et al., 2015). NAFLD patients also experience anxiety (Mahmood et al., 2008) and cognitive impairment (Filipović et al., 2018) more often than the general population. Children with autism show an increased risk of NAFLD development (Shedlock et al., 2016).Cumulatively, this points to a strong association between metabolic and psychiatric disorders, and in many cases there are indications of a bidirectional relationship. It can be hypothesized that the presence of one condition may exacerbate the symptoms of another due to common pathophysiological mechanisms. Moreover, both metabolic and psychiatric disorders may share predisposing lifestyle factors or genetic susceptibility. 


\subsection{Role of decreased function of the serotonin transporter (SERT) in metabolic and mental disorders}

The serotonin transporter (SERT) plays a central role in the regulation of serotonergic neurotransmission (Collier et al., 1996; Murphy et al., 2004) and is implicated in the regulation of metabolic processes as well as mechanisms of psychiatric disorders (Homberg and Lesch, 2011; Margoob and Mushtaq, 2011). In humans, two key variants in the regulatory region (5-HTTLPR) of the SERT (SLC6A4) gene exist, the so-called short (s) allele, which in comparison with the long (l) allele, reduces the expression of SERT. This is associated with higher body mass index (BMI) (Fuemmeler et al., 2008; Sookoian et al., 2007) and incidence of T2D (Iordanidou et al., 2010). Downregulation of SERT in platelet membranes (Giannaccini et al., 2013) and cerebellum (Erritzoe et al., 2010) is also shown in obese people. Excessive intake of a hypercaloric diet enriched with saturated fat and sugars is shown to suppress the binding of hypothalamic SERT in obese subjects and diencephalon SERT in insulin-resistant subjects (Koopman et al., 2013; Versteeg et al., 2017).

Carriers of the s allele were also reported to be more susceptible to development of anxiety-related traits (Lesch et al., 1996) and depression (Caspi et al., 2003) in the context of adverse environmental challenge, although these findings have controversially been discussed in recent years. Multiple studies have shown that $S L C 6 A 4$ represents 
a susceptibility locus for autism-spectrum disorders (Sutcliffe et al., 2005). Studies of 5-HTTLPR association with autism has yielded inconsistent results, with associations shown mostly to the s allele (Cook et al., 1997; Devlin et al., 2005; Tordjman et al., 2001) but also some to 1 allele (Yirmiya et al., 2001). Autism is an extremely heterogeneous disease with numerous single nucleotide polymorphisms (SNPs) found in ASD patients, which makes it possible that multiple different alleles at SLC6A4 contribute to the genetic risk of the disorder (Devlin et al., 2005; McCauley et al., 2004). Genome-wide linkage scans have demonstrated a positive linkage of ADHD and SERT (Fisher et al., 2002). Reduced availability of SERT in the brain is shown in patients with ADHD and conduct disorder (Chang et al., 2017; Vanicek et al., 2017).

Decreased SERT functioning was proposed to contribute to the development of both mental and metabolic disorders through elevated LPS levels and increased production of proinflammatory cytokines in the periphery (Pomytkin et al., 2015). Uptake by SERT is a key mechanism controlling the availability of serotonin in the gastrointestinal tract (Racké et al., 1996), meaning that decreased SERT would lead to excessive extracellular serotonin concentrations and increased gut permeability mediated by the 5-HT3 receptor (Yamada et al., 2003) (see section 1.2). This hypothesis was supported by a study in Sert knockout mice which demonstrated enhanced 
intestinal permeability and LPS leakage (El Aidy et al., 2017). If translated to humans, the 5-HTTLPR might result in similar effects related to endotoxemia and subsequent peripheral inflammation, which could contribute to the mechanisms of susceptibility to mental and metabolic disorders.

Thus, such overlapping psychiatric disorders as ADHD, ASD and depression (Taurines et al., 2012), as well as metabolic disorders have shared genetic susceptibility that could possibly interact with environmental factors, for example, diet. However, the mechanisms of the interplay between genetic SERT deficiency and metabolic dysregulation need further investigation.

\subsection{Studying the effects of Western diet on brain functions using animal models}

Animal feed enriched with fat, sugars and cholesterol, which mimics some aspects of a human Western dietary pattern, is typically used to study the mechanisms of diet on systemic physiology in vivo. Western diet-fed rodents are widely and successfully used to model such conditions as obesity, metabolic disorder, and insulin resistance (Hariri and Thibault, 2010; Hintze et al., 2018). More recently, Western diet-feeding in animals has been employed to investigate the nutritional consequences on behaviour and brain functioning.

Considerable variation exists in the use of, and experimental conditions associated with Western diet analogs in research (Fig. 1.6). 
Fat content usually varies between $40 \%$ and $75 \%$ of total calories (Hintze et al., 2018), and diet composition may include different animal-derived fats or plant oils, which is known to elicit distinct effects on physiology (Buettner et al., 2006; Hryhorczuk et al., 2013). Duration of exposure to Western diet may vary from 1 week (Kaczmarczyk et al., 2013) to several months (André et al., 2014; Yamada et al., 2011). The age at which Western diet is administered also varies, with experiments utilizing young adult (Papazoglou et al., 2015; Strekalova et al., 2015, 2016; Yamada et al., 2011), aging (Kesby et al., 2015), or juvenile mice and rats (André et al., 2014; Kaczmarczyk et al., 2013). Exposure can also start in the early postnatal period (Carlin et al., 2013) or prenatally (Buffington et al., 2016). Different models involving adult and juvenile rodents provide an opportunity to explore diet-induced effects both on mature and developing organisms.

Peripheral changes during exposure to the Western diet differ between sexes, possibly contributing to the difference in dietary effects on the CNS. Male mice display faster body weight increases and greater dyslipidemia while female mice are more susceptible to liver steatosis and inflammation (Comhair et al., 2011; Dong et al., 2018; Gasparin et al., 2018; Homberg et al., 2010; Li et al., 2017; Link et al., 2015). Sex differences in the metabolic effects of diet-induced obesity were also shown to vary by age of onset of dietary intervention. In juvenile 
mice the Western diet caused a greater increase in body weight and greater impairment in glucose tolerance in males than in female mice (Freire-Regatillo et al., 2019; Salinero et al., 2018). In later in life, when mice were 32 weeks old, the sex difference was reversed (Salinero et al., 2018). Despite the fact that the number of women affected by mental disorders is very high, the current literature on dietary effects on males is more abundant than the effects of diet on females.

\section{Models of Western diet feeding}
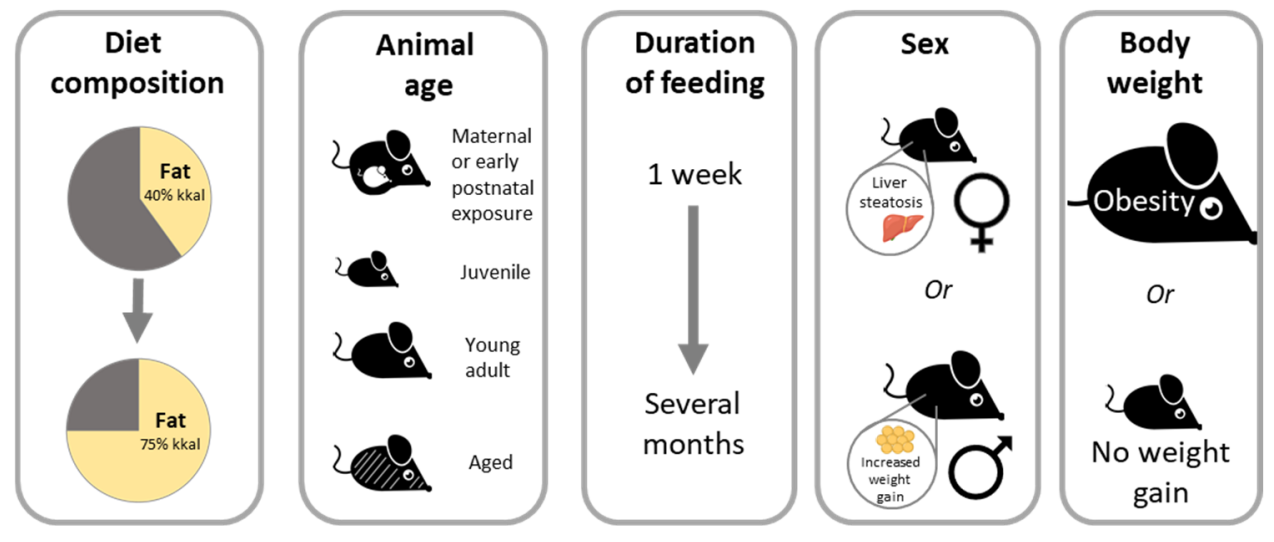

\section{Different effects on the brain functioning}

Figure 1.6. Variable parameters in animal models of dietary exposure. Models may vary in diet composition, animal age of dietary intervention onset, duration of feeding, animal sex, and effects of the diet on body weight. 
Many studies have shown cognitive deficit, depressive-like behaviours and anxiety in diet-induced obesity in rodents (Abbott et al., 2019; André et al., 2014; Carlin et al., 2013; Nguyen et al., 2014a; Papazoglou et al., 2015; Vagena et al., 2019). This is associated with neuroinflammation, oxidative stress, changes in metabolome and BBB permeability as well as alterations in brain signaling systems. However, it remains unclear as to whether the dietary effects on CNS functioning in obesity and in non-obesogenic Western diet feeding have similar mechanisms. In addition, increased body weight in animal models can make it difficult to interpret test results.

Few studies have used non-obese animals fed with Western diet for a short period of time to examine behavioural and molecular brain changes. For example, memory deficit were reported in rats fed with Western diet without developing obesity using an 8-arm radial maze test of hippocampus-dependent working memory (Granholm et al., 2008; Kanoski and Davidson, 2010; Murray et al., 2009). It was shown that Western diet feeding for 1 week caused anxiety-like behaviors and impaired memory while body weight gain was significant only after 3 weeks of the diet feeding (Kaczmarczyk et al., 2013). Young female mice fed with the Western diet for 3 weeks demonstrated anxiety and depressive-like behaviour accompanied by TLR4 activation and constant body weight (Strekalova et al., 2015). 
While impairments in social behaviours and motor coordination are important components of some disorders including ASD and ADHD, few studies have examined the effects of Western diet on these behaviours in animal models. One study using long-term high fat diet feeding in mice showed time-dependent reduction in motor coordinative skills measured by rotarod, pole test, stepping test and hind-limb clasping test. Muscle strength, locomotor activity and body weight were unaffected (Stojakovic et al., 2018). Social behaviours have mainly been studied in juvenile mice and rats, showing that Western diet can induce social recognition deficits (Buffington et al., 2016; Reichelt et al., 2019). Rodent models allow us to study not only the effects of the Western diet on behaviours reminiscent of mental disorder symptomology, but also to explore the mechanisms underlying behavioural changes.

\section{Hypothesis and aim}

In this study we used a model in which female C57BL/6J mice are fed with the Western diet for three weeks. Based on the previous experiments in this model, which revealed increased anxiety and depressive-like behaviour in young mice housed on the Western diet, and human data regarding cooccurrence of ASD and ADHD with depression and impaired metabolism, we hypothesized that Western diet may affect behaviours associated with these disorders. We aimed 
to investigate the effects of the Western diet on social interactions, cognitive functions, emotionality and motor coordination (Chapters 2 and 3). In addition, we investigated diet-induced metabolic changes which are relevant to the development of behavioural abnormalities including glucose tolerance and insulin resistance, blood levels of lipids, liver injury markers and liver steatosis (Chapters 2 and 3). To explore the mechanisms underlying behavioural changes, we studied lipid peroxidation, microglial activation and gene expression of the mitochondrial biogenesis marker, and expression of the serotonin transporter in the brain of mice fed with the Western diet (Chapters

\section{2 and 3).}

Based on the well-established relationship between genetic deficit in SERT activity and susceptibility to mental and metabolic aberrations in humans, we next studied how Western diet, which in our model decreases Sert expression (Chapter 3), interferes with genetic Sert deficiency in mice (Chapter 4). It was hypothesized that complete and partial absence of Sert may differentially affect Western diet-induced changes. Using mice with a heterozygous and complete knockout for the Sert gene, we investigated diet-induced behavioural, metabolic changes and analyzed them in connection to molecular changes in the brain. 


\section{References}

Abbott, K.N., Arnott, C.K., Westbrook, R.F., Tran, D.M.D., 2019. The effect of high fat, high sugar, and combined high fat-high sugar diets on spatial learning and memory in rodents: A meta-analysis. Neurosci. Biobehav. Rev. 107, 399-421. https://doi.org/10.1016/j.neubiorev.2019.08.010

Adriaanse, M.C., Dekker, J.M., Heine, R.J., Snoek, F.J., Beekman, A.J., Stehouwer, C.D., Bouter, L.M., Nijpels, G., Pouwer, F., 2008. Symptoms of depression in people with impaired glucose metabolism or Type2 diabetes mellitus: The Hoorn Study. Diabet. Med. 25, 843-849. https://doi.org/10.1111/j.1464-5491.2008.02464.x

Aguirre, V., Uchida, T., Yenush, L., Davis, R., White, M.F., 2000. The cJun NH 2 -terminal Kinase Promotes Insulin Resistance during Association with Insulin Receptor Substrate-1 and Phosphorylation of Ser 307. J. Biol. Chem. 275, 9047-9054. https://doi.org/10.1074/jbc.275.12.9047

Akbaraly, T.N., Brunner, E.J., Ferrie, J.E., Marmot, M.G., Kivimaki, M., Singh-Manoux, A., 2009. Dietary pattern and depressive symptoms in middle age. Br. J. Psychiatry 195, 408-413.

https://doi.org/10.1192/bjp.bp.108.058925

Aksenova, M. V, Aksenov, M.Y., Mactutus, C.F., Booze, R.M., 2005. Cell culture models of oxidative stress and injury in the central nervous system. Curr. Neurovasc. Res. 2, 73-89.

Allison, D.J., Ditor, D.S., 2014. The common inflammatory etiology of depression and cognitive impairment: a therapeutic target. J. Neuroinflammation 11, 151. https://doi.org/10.1186/s12974-014-0151-1

Altfas, J.R., 2002. Prevalence of attention deficit/hyperactivity disorder among adults in obesity treatment. BMC Psychiatry 2, 9.

Andersen, C.H., Thomsen, P.H., Nohr, E.A., Lemcke, S., 2018. Maternal body mass index before pregnancy as a risk factor for ADHD and autism in children. Eur. Child Adolesc. Psychiatry 27, 139-148.

https://doi.org/10.1007/s00787-017-1027-6

André, C., Dinel, A.-L., Ferreira, G., Layé, S., Castanon, N., 2014. Dietinduced obesity progressively alters cognition, anxiety-like behavior and lipopolysaccharide-induced depressive-like behavior: focus on brain indoleamine 2,3-dioxygenase activation. Brain. Behav. Immun. 41, 10-21. https://doi.org/10.1016/j.bbi.2014.03.012

Anil Kumar, B., Malhotra, S., Bhattacharya, A., Grover, S., Batra, Y., 2017. Regional cerebral glucose metabolism and its association with 
phenotype and cognitive functioning in patients with autism. Indian J. Psychol. Med. 39, 262. https://doi.org/10.4103/0253-7176.207344

Bajwa, W., Asnis, G., Sanderson, W., Irfan, A., van Praag, H., 1992. High cholesterol levels in patients with panic disorder. Am. J. Psychiatry 149, 376378. https://doi.org/10.1176/ajp.149.3.376

Banks, W.A., Coon, A.B., Robinson, S.M., Moinuddin, A., Shultz, J.M., Nakaoke, R., Morley, J.E., 2004. Triglycerides induce leptin resistance at the blood-brain barrier. Diabetes 53, 1253-60.

https://doi.org/10.2337/diabetes.53.5.1253

Banks, W.A., Erickson, M.A., 2010. The blood-brain barrier and immune function and dysfunction. Neurobiol. Dis. 37, 26-32.

https://doi.org/10.1016/j.nbd.2009.07.031

Banks, W.A., Kastin, A.J., Broadwell, R.D., 1995. Passage of cytokines across the blood-brain barrier. Neuroimmunomodulation 2, 241-8. https://doi.org/10.1159/000097202

Banks, W.A., Niehoff, M.L., Ponzio, N.M., Erickson, M.A., Zalcman, S.S., 2012. Pharmacokinetics and modeling of immune cell trafficking: quantifying differential influences of target tissues versus lymphocytes in SJL and lipopolysaccharide-treated mice. J. Neuroinflammation 9, 231. https://doi.org/10.1186/1742-2094-9-231

Bastard, J.-P., Maachi, M., Lagathu, C., Kim, M.J., Caron, M., Vidal, H., Capeau, J., Feve, B., 2006. Recent advances in the relationship between obesity, inflammation, and insulin resistance. Eur. Cytokine Netw. 17, 4-12.

Bechmann, I., Galea, I., Perry, V.H., 2007. What is the blood-brain barrier (not)? Trends Immunol. 28, 5-11. https://doi.org/10.1016/j.it.2006.11.007

Bleau, C., Karelis, A.D., St-Pierre, D.H., Lamontagne, L., 2015. Crosstalk between intestinal microbiota, adipose tissue and skeletal muscle as an early event in systemic low-grade inflammation and the development of obesity and diabetes. Diabetes. Metab. Res. Rev. 31, 545-61. https://doi.org/10.1002/dmrr.2617

Bodenlos, J.S., Lemon, S.C., Schneider, K.L., August, M.A., Pagoto, S.L., 2011. Associations of mood and anxiety disorders with obesity: Comparisons by ethnicity. J. Psychosom. Res. 71, 319-324.

https://doi.org/10.1016/j.jpsychores.2011.03.004

Bogoyevitch, M.A., Kobe, B., 2006. Uses for JNK: the Many and Varied Substrates of the c-Jun N-Terminal Kinases. Microbiol. Mol. Biol. Rev. 70, 1061-1095. https://doi.org/10.1128/MMBR.00025-06

Bohatschek, M., Werner, A., Raivich, G., 2001. Systemic LPS injection 
leads to granulocyte influx into normal and injured brain: effects of ICAM-1 deficiency. Exp. Neurol. 172, 137-52. https://doi.org/10.1006/exnr.2001.7764

Bouwman, V., Adriaanse, M.C., van 't Riet, E., Snoek, F.J., Dekker, J.M., Nijpels, G., 2010. Depression, Anxiety and Glucose Metabolism in the General Dutch Population: The New Hoorn Study. PLoS One 5, e9971. https://doi.org/10.1371/journal.pone.0009971

Brand-Miller, J.C., Thomas, M., Swan, V., Ahmad, Z.I., Petocz, P., Colagiuri, S., 2003. Physiological Validation of the Concept of Glycemic Load in Lean Young Adults. J. Nutr. 133, 2728-2732.

https://doi.org/10.1093/jn/133.9.2728

Bray, G.A., Popkin, B.M., 2014. Dietary Sugar and Body Weight: Have We Reached a Crisis in the Epidemic of Obesity and Diabetes? Diabetes Care 37, 950-956. https://doi.org/10.2337/dc13-2085

Buettner, R., Parhofer, K.G., Woenckhaus, M., Wrede, C.E., KunzSchughart, L.A., Schölmerich, J., Bollheimer, L.C., 2006. Defining high-fatdiet rat models: metabolic and molecular effects of different fat types. J. Mol. Endocrinol. 36, 485-501. https://doi.org/10.1677/jme.1.01909

Buffington, S.A., Di Prisco, G.V., Auchtung, T.A., Ajami, N.J., Petrosino, J.F., Costa-Mattioli, M., 2016. Microbial Reconstitution Reverses Maternal Diet-Induced Social and Synaptic Deficits in Offspring. Cell 165, 1762-1775. https://doi.org/10.1016/j.cell.2016.06.001

Butwicka, A., Frisén, L., Almqvist, C., Zethelius, B., Lichtenstein, P., 2015. Risks of Psychiatric Disorders and Suicide Attempts in Children and Adolescents With Type 1 Diabetes: A Population-Based Cohort Study. Diabetes Care 38, 453-459. https://doi.org/10.2337/dc14-0262

Caesar, R., Tremaroli, V., Kovatcheva-Datchary, P., Cani, P.D., Bäckhed, F., 2015. Crosstalk between Gut Microbiota and Dietary Lipids Aggravates WAT Inflammation through TLR Signaling. Cell Metab. 22, 658-68. https://doi.org/10.1016/j.cmet.2015.07.026

Capuron, L., Pagnoni, G., Drake, D.F., Woolwine, B.J., Spivey, J.R., Crowe, R.J., Votaw, J.R., Goodman, M.M., Miller, A.H., 2012. Dopaminergic mechanisms of reduced basal ganglia responses to hedonic reward during interferon alfa administration. Arch. Gen. Psychiatry 69, 1044-53. https://doi.org/10.1001/archgenpsychiatry.2011.2094

Carlin, J., Hill-Smith, T.E., Lucki, I., Reyes, T.M., 2013. Reversal of dopamine system dysfunction in response to high-fat diet. Obesity (Silver Spring). 21, 2513-21. https://doi.org/10.1002/oby.20374

Carrard, A., Elsayed, M., Margineanu, M., Boury-Jamot, B., Fragnière, 
L., Meylan, E.M., Petit, J.-M., Fiumelli, H., Magistretti, P.J., Martin, J.-L., 2016. Peripheral administration of lactate produces antidepressant-like effects. Mol. Psychiatry 23, 392.

Carrera-Bastos, P., Fontes, O'Keefe, Lindeberg, Cordain, 2011. The western diet and lifestyle and diseases of civilization. Res. Reports Clin. Cardiol. 15. https://doi.org/10.2147/RRCC.S16919

Chang, C., Gau, S.S.-F., Huang, W.-S., Shiue, C.-Y., Yeh, C.-B., 2017. Abnormal serotonin transporter availability in the brains of adults with conduct disorder. J. Formos. Med. Assoc. 116, 469-475.

https://doi.org/10.1016/j.jfma.2016.07.012

Cline, B.H., Steinbusch, H.W., Malin, D., Revishchin, A. V, Pavlova, G. V, Cespuglio, R., Strekalova, T., 2012. The neuronal insulin sensitizer dicholine succinate reduces stress-induced depressive traits and memory deficit: possible role of insulin-like growth factor 2. BMC Neurosci. 13, 110. https://doi.org/10.1186/1471-2202-13-110

Codoñer-Franch, P., Tavárez-Alonso, S., Murria-Estal, R., MegíasVericat, J., Tortajada-Girbés, M., Alonso-Iglesias, E., 2011. Nitric oxide production is increased in severely obese children and related to markers of oxidative stress and inflammation. Atherosclerosis 215, 475-80. https://doi.org/10.1016/j.atherosclerosis.2010.12.035

Comhair, T.M., Garcia Caraballo, S.C., Dejong, C.H., Lamers, W.H., Koehler, S.E., 2011. Dietary cholesterol, female gender and n-3 fatty acid deficiency are more important factors in the development of non-alcoholic fatty liver disease than the saturation index of the fat. Nutr. Metab. (Lond). 8, 4. https://doi.org/10.1186/1743-7075-8-4

Connolly, N., Anixt, J., Manning, P., Ping-I Lin, D., Marsolo, K.A., Bowers, K., 2016. Maternal metabolic risk factors for autism spectrum disorder-An analysis of electronic medical records and linked birth data. Autism Res. 9, 829-837. https://doi.org/10.1002/aur.1586

Cook, E., Courchesne, R., Lord, C., Cox, N.J., Yan, S., Lincoln, A., Haas, R., Courchesne, E., Leventhal, B.L., 1997. Evidence of linkage between the serotonin transporter and autistic disorder. Mol. Psychiatry 2, 247-250. https://doi.org/10.1038/sj.mp.4000266

Cordain, L., Eaton, S.B., Sebastian, A., Mann, N., Lindeberg, S., Watkins, B.A., O'Keefe, J.H., Brand-Miller, J., 2005. Origins and evolution of the Western diet: health implications for the 21st century. Am. J. Clin. Nutr. 81, 341-354. https://doi.org/10.1093/ajcn.81.2.341

Cortese, S., Konofal, E., Bernardina, B.D., Mouren, M.-C., Lecendreux, 
M., 2008. Does excessive daytime sleepiness contribute to explaining the association between obesity and ADHD symptoms? Med. Hypotheses 70, 12 16. https://doi.org/Cortese

Cortese, S., Tessari, L., 2017. Attention-Deficit/Hyperactivity Disorder (ADHD) and Obesity: Update 2016. Curr. Psychiatry Rep. 19, 4. https://doi.org/10.1007/s11920-017-0754-1

Dantzer, R., O'Connor, J.C., Freund, G.G., Johnson, R.W., Kelley, K.W., 2008. From inflammation to sickness and depression: when the immune system subjugates the brain. Nat. Rev. Neurosci. 9, 46-56. https://doi.org/10.1038/nrn2297

Devlin, B., Cook, E.H., Coon, H., Dawson, G., Grigorenko, E.L., McMahon, W., Minshew, N., Pauls, D., Smith, M., Spence, M.A., Rodier, P.M., Stodgell, C., Schellenberg, G.D., 2005. Autism and the serotonin transporter: the long and short of it. Mol. Psychiatry 10, 1110-1116. https://doi.org/10.1038/sj.mp.4001724

Ding, S., Chi, M.M., Scull, B.P., Rigby, R., Schwerbrock, N.M.J., Magness, S., Jobin, C., Lund, P.K., 2010. High-fat diet: bacteria interactions promote intestinal inflammation which precedes and correlates with obesity and insulin resistance in mouse. PLoS One 5, e12191. https://doi.org/10.1371/journal.pone.0012191

Dong, Q., Kuefner, M.S., Deng, X., Bridges, D., Park, E.A., Elam, M.B., Raghow, R., 2018. Sex-specific differences in hepatic steatosis in obese spontaneously hypertensive (SHROB) rats. Biol. Sex Differ. 9, 40. https://doi.org/10.1186/s13293-018-0202-x

Drewnowski, A., 2007. The Real Contribution of Added Sugars and Fats to Obesity. Epidemiol. Rev. 29, 160-171.

https://doi.org/10.1093/epirev/mxm011

El Aidy, S., Ramsteijn, A.S., Dini-Andreote, F., van Eijk, R., Houwing, D.J., Salles, J.F., Olivier, J.D.A., 2017. Serotonin Transporter Genotype Modulates the Gut Microbiota Composition in Young Rats, an Effect Augmented by Early Life Stress. Front. Cell. Neurosci. 11, 222. https://doi.org/10.3389/fncel.2017.00222

Elmarakby, A.A., Sullivan, J.C., 2012. Relationship between oxidative stress and inflammatory cytokines in diabetic nephropathy. Cardiovasc. Ther. 30, 49-59. https://doi.org/10.1111/j.1755-5922.2010.00218.x

Elwing, J.E., Lustman, P.J., Wang, H.L., Clouse, R.E., 2006. Depression, Anxiety, and Nonalcoholic Steatohepatitis. Psychosom. Med. 68, 563-569. https://doi.org/10.1097/01.psy.0000221276.17823.df 
Erritzoe, D., Frokjaer, V.G., Haahr, M.T., Kalbitzer, J., Svarer, C., Holst, K.K., Hansen, D.L., Jernigan, T.L., Lehel, S., Knudsen, G.M., 2010. Cerebral serotonin transporter binding is inversely related to body mass index. Neuroimage 52, 284-289. https://doi.org/10.1016/j.neuroimage.2010.03.086

Filipović, Branka, Marković, O., Đurić, V., Filipović, Branislav, 2018. Cognitive Changes and Brain Volume Reduction in Patients with Nonalcoholic Fatty Liver Disease. Can. J. Gastroenterol. Hepatol. 2018, 1-6. https://doi.org/10.1155/2018/9638797

Firth, J., Stubbs, B., Teasdale, S.B., Ward, P.B., Veronese, N., Shivappa, N., Hebert, J.R., Berk, M., Yung, A.R., Sarris, J., 2018. Diet as a hot topic in psychiatry: a population-scale study of nutritional intake and inflammatory potential in severe mental illness. World Psychiatry 17, 365367. https://doi.org/10.1002/wps. 20571

Fisher, S.E., Francks, C., McCracken, J.T., McGough, J.J., Marlow, A.J., MacPhie, I.L., Newbury, D.F., Crawford, L.R., Palmer, C.G.S., Woodward, J.A., Del'Homme, M., Cantwell, D.P., Nelson, S.F., Monaco, A.P., Smalley, S.L., 2002. A Genomewide Scan for Loci Involved in AttentionDeficit/Hyperactivity Disorder. Am. J. Hum. Genet. 70, 1183-1196. https://doi.org/10.1086/340112

Fleming, J.P., Levy, L.D., Levitan, R.D., 2005. Symptoms of attention deficit hyperactivity disorder in severely obese women. Eat. Weight Disord. 10, e10-3.

Freire-Regatillo, A., Fernández-Gómez, M.J., Díaz, F., Barrios, V., Sánchez-Jabonero, I., Frago, L.M., Argente, J., García-Segura, L.M., Chowen, J.A., 2019. Sex differences in the peripubertal response to a shortterm, high-fat diet intake. J. Neuroendocrinol.

https://doi.org/10.1111/jne.12756

Fuhrer, R., Keyes, K.M., 2019. Population Mental Health in the 21st Century: Aspirations and Experiences. Am. J. Public Health 109, S150-S151. https://doi.org/10.2105/AJPH.2019.305187

Fung, T.T., Schulze, M., Manson, J.E., Willett, W.C., Hu, F.B., 2004. Dietary Patterns, Meat Intake, and the Risk of Type 2 Diabetes in Women. Arch. Intern. Med. 164, 2235. https://doi.org/10.1001/archinte.164.20.2235

Furukawa, S., Fujita, T., Shimabukuro, M., Iwaki, M., Yamada, Y., Nakajima, Y., Nakayama, O., Makishima, M., Matsuda, M., Shimomura, I., 2004. Increased oxidative stress in obesity and its impact on metabolic syndrome. J. Clin. Invest. 114, 1752-61. https://doi.org/10.1172/JCI21625

Gami, A.S., Caples, S.M., Somers, V.K., 2003. Obesity and obstructive 
sleep apnea. Endocrinol. Metab. Clin. North Am. 32, 869-894. https://doi.org/10.1016/S0889-8529(03)00069-0

Gasparin, F.R.S., Carreño, F.O., Mewes, J.M., Gilglioni, E.H., Pagadigorria, C.L.S., Natali, M.R.M., Utsunomiya, K.S., Constantin, R.P., Ouchida, A.T., Curti, C., Gaemers, I.C., Elferink, R.P.J.O., Constantin, J., Ishii-Iwamoto, E.L., 2018. Sex differences in the development of hepatic steatosis in cafeteria diet-induced obesity in young mice. Biochim. Biophys. Acta - Mol. Basis Dis. 1864, 2495-2509.

https://doi.org/10.1016/j.bbadis.2018.04.004

Gillberg, C., Fernell, E., Kočovská, E., Minnis, H., Bourgeron, T., Thompson, L., Allely, C.S., 2017. The role of cholesterol metabolism and various steroid abnormalities in autism spectrum disorders: A hypothesis paper. Autism Res. 10, 1022-1044. https://doi.org/10.1002/aur.1777

Granholm, A.-C., Bimonte-Nelson, H.A., Moore, A.B., Nelson, M.E., Freeman, L.R., Sambamurti, K., 2008. Effects of a saturated fat and high cholesterol diet on memory and hippocampal morphology in the middle-aged rat. J. Alzheimers. Dis. 14, 133-45.

Gustafson, D.R., Karlsson, C., Skoog, I., Rosengren, L., Lissner, L., Blennow, K., 2007. Mid-life adiposity factors relate to blood-brain barrier integrity in late life. J. Intern. Med. 262, 643-50.

https://doi.org/10.1111/j.1365-2796.2007.01869.x

Hariri, N., Thibault, L., 2010. High-fat diet-induced obesity in animal models. Nutr. Res. Rev. 23, 270-99.

https://doi.org/10.1017/S0954422410000168

Haroon, E., Raison, C.L., Miller, A.H., 2012. Psychoneuroimmunology meets neuropsychopharmacology: translational implications of the impact of inflammation on behavior. Neuropsychopharmacology 37, 137-62. https://doi.org/10.1038/npp.2011.205

Hintze, K.J., Benninghoff, A.D., Cho, C.E., Ward, R.E., 2018. Modeling the Western Diet for Preclinical Investigations. Adv. Nutr. 9, 263-271. https://doi.org/10.1093/advances/nmy002

Homberg, J.R., la Fleur, S.E., Cuppen, E., 2010. Serotonin Transporter Deficiency Increases Abdominal Fat in Female, but Not Male Rats. Obesity 18, 137-145. https://doi.org/10.1038/oby.2009.139

Homberg, J.R., Lesch, K.-P., 2011. Looking on the bright side of serotonin transporter gene variation. Biol. Psychiatry 69, 513-9.

https://doi.org/10.1016/j.biopsych.2010.09.024

Hosseini, Z., Whiting, S.J., Vatanparast, H., 2016. Current evidence on 
the association of the metabolic syndrome and dietary patterns in a global perspective. Nutr. Res. Rev. 29, 152-162.

https://doi.org/10.1017/S095442241600007X

Howard, A.L., Robinson, M., Smith, G.J., Ambrosini, G.L., Piek, J.P., Oddy, W.H., 2011. ADHD Is Associated With a "Western" Dietary Pattern in Adolescents. J. Atten. Disord. 15, 403-411.

https://doi.org/10.1177/1087054710365990

Hryhorczuk, C., Sharma, S., Fulton, S.E., 2013. Metabolic disturbances connecting obesity and depression. Front. Neurosci. 7, 177.

https://doi.org/10.3389/fnins.2013.00177

Ida, T., Hara, M., Nakamura, Y., Kozaki, S., Tsunoda, S., Ihara, H., 2008. Cytokine-induced enhancement of calcium-dependent glutamate release from astrocytes mediated by nitric oxide. Neurosci. Lett. 432, 232-6. https://doi.org/10.1016/j.neulet.2007.12.047

Ipsen, D.H., Lykkesfeldt, J., Tveden-Nyborg, P., 2018. Molecular mechanisms of hepatic lipid accumulation in non-alcoholic fatty liver disease. Cell. Mol. Life Sci. 75, 3313-3327. https://doi.org/10.1007/s00018-018-2860-6

Jacka, F.N., O'Neil, A., Opie, R., Itsiopoulos, C., Cotton, S., Mohebbi, M., Castle, D., Dash, S., Mihalopoulos, C., Chatterton, M. Lou, Brazionis, L., Dean, O.M., Hodge, A.M., Berk, M., 2017. A randomised controlled trial of dietary improvement for adults with major depression (the 'SMILES' trial). BMC Med. 15, 23. https://doi.org/10.1186/s12916-017-0791-y

Jacka, F.N., Pasco, J.A., Mykletun, A., Williams, L.J., Hodge, A.M., O'Reilly, S.L., Nicholson, G.C., Kotowicz, M.A., Berk, M., 2010. Association of Western and Traditional Diets With Depression and Anxiety in Women. Am. J. Psychiatry 167, 305-311.

https://doi.org/10.1176/appi.ajp.2009.09060881

Ji, Y., Riley, A.W., Lee, L.-C., Volk, H., Hong, X., Wang, G., Angomas, R., Stivers, T., Wahl, A., Ji, H., Bartell, T.R., Burd, I., Paige, D., Fallin, M.D., Zuckerman, B., Wang, X., 2017. A Prospective Birth Cohort Study on Maternal Cholesterol Levels and Offspring Attention Deficit Hyperactivity Disorder: New Insight on Sex Differences. Brain Sci. 8. https://doi.org/10.3390/brainsci8010003

Kaczmarczyk, M.M., Machaj, A.S., Chiu, G.S., Lawson, M.A., Gainey, S.J., York, J.M., Meling, D.D., Martin, S.A., Kwakwa, K.A., Newman, A.F., Woods, J.A., Kelley, K.W., Wang, Y., Miller, M.J., Freund, G.G., 2013. Methylphenidate prevents high-fat diet (HFD)-induced learning/memory impairment in juvenile mice. Psychoneuroendocrinology 38, 1553-64. 
https://doi.org/10.1016/j.psyneuen.2013.01.004

Kaiyala, K.J., Prigeon, R.L., Kahn, S.E., Woods, S.C., Schwartz, M.W., 2000. Obesity induced by a high-fat diet is associated with reduced brain insulin transport in dogs. Diabetes 49, 1525-1533. https://doi.org/10.2337/diabetes.49.9.1525

Kanoski, S.E., Davidson, T.L., 2010. Different patterns of memory impairments accompany short- and longer-term maintenance on a highenergy diet. J. Exp. Psychol. Anim. Behav. Process. 36, 313-319. https://doi.org/10.1037/a0017228

Kant, A.K., 2004. Dietary patterns and health outcomes. J. Am. Diet. Assoc. 104, 615-635. https://doi.org/10.1016/j.jada.2004.01.010

Karin, M., Gallagher, E., 2005. From JNK to Pay Dirt: Jun Kinases, their Biochemistry, Physiology and Clinical Importance. IUBMB Life (International Union Biochem. Mol. Biol. Life) 57, 283-295. https://doi.org/10.1080/15216540500097111

Kazumori, H., Ishihara, S., Rumi, M.A.K., Ortega-Cava, C.F., Kadowaki, Y., Kinoshita, Y., 2004. Transforming growth factor-alpha directly augments histidine decarboxylase and vesicular monoamine transporter 2 production in rat enterochromaffin-like cells. Am. J. Physiol. Gastrointest. Liver Physiol. 286, G508-14. https://doi.org/10.1152/ajpgi.00269.2003

Kesby, J.P., Kim, J.J., Scadeng, M., Woods, G., Kado, D.M., Olefsky, J.M., Jeste, D. V, Achim, C.L., Semenova, S., 2015. Spatial Cognition in Adult and Aged Mice Exposed to High-Fat Diet. PLoS One 10, e0140034. https://doi.org/10.1371/journal.pone.0140034

Kim, E.-K., Neggers, Y.H., Shin, C.-S., Kim, E., Kim, E.M., 2010. Alterations in lipid profile of autistic boys: a case control study. Nutr. Res. 30, 255-260. https://doi.org/10.1016/j.nutres.2010.04.002

Kim, K.-A., Gu, W., Lee, I.-A., Joh, E.-H., Kim, D.-H., 2012. High fat diet-induced gut microbiota exacerbates inflammation and obesity in mice via the TLR4 signaling pathway. PLoS One 7, e47713.

https://doi.org/10.1371/journal.pone.0047713

Kim, M., Cooke, H.J., Javed, N.H., Carey, H. V, Christofi, F., Raybould, H.E., 2001. D-glucose releases 5-hydroxytryptamine from human BON cells as a model of enterochromaffin cells. Gastroenterology 121, 1400-6. https://doi.org/10.1053/gast.2001.29567

Kleinridders, A., Cai, W., Cappellucci, L., Ghazarian, A., Collins, W.R., Vienberg, S.G., Pothos, E.N., Kahn, C.R., 2015. Insulin resistance in brain alters dopamine turnover and causes behavioral disorders. Proc. Natl. Acad. 
Sci. U. S. A. 112, 3463-8. https://doi.org/10.1073/pnas.1500877112

Koponen, H., Kautiainen, H., Leppänen, E., Mäntyselkä, P., Vanhala, M., 2015. Association between suicidal behaviour and impaired glucose metabolism in depressive disorders. BMC Psychiatry 15, 163. https://doi.org/10.1186/s12888-015-0567-x

Kozler, P., Pokorný, J., 2003. Altered blood-brain barrier permeability and its effect on the distribution of Evans blue and sodium fluorescein in the rat brain applied by intracarotid injection. Physiol. Res. 52, 607-14.

Krakowiak, P., Walker, C.K., Bremer, A.A., Baker, A.S., Ozonoff, S., Hansen, R.L., Hertz-Picciotto, I., 2012. Maternal Metabolic Conditions and Risk for Autism and Other Neurodevelopmental Disorders. Pediatrics 129, e1121-e1128. https://doi.org/10.1542/peds.2011-2583

Lafontan, M., 2014. Adipose tissue and adipocyte dysregulation. Diabetes Metab. 40, 16-28. https://doi.org/10.1016/j.diabet.2013.08.002

Le Chatelier, E., Nielsen, T., Qin, J., Prifti, E., Hildebrand, F., Falony, G., Almeida, M., Arumugam, M., Batto, J.-M., Kennedy, S., Leonard, P., Li, J., Burgdorf, K., Grarup, N., Jørgensen, T., Brandslund, I., Nielsen, H.B., Juncker, A.S., Bertalan, M., Levenez, F., Pons, N., Rasmussen, S., Sunagawa, S., Tap, J., Tims, S., Zoetendal, E.G., Brunak, S., Clément, K., Doré, J., Kleerebezem, M., Kristiansen, K., Renault, P., Sicheritz-Ponten, T., de Vos, W.M., Zucker, J.-D., Raes, J., Hansen, T., MetaHIT consortium, Bork, P., Wang, J., Ehrlich, S.D., Pedersen, O., 2013. Richness of human gut microbiome correlates with metabolic markers. Nature 500, 541-6. https://doi.org/10.1038/nature12506

Le Lay, S., Simard, G., Martinez, M.C., Andriantsitohaina, R., 2014. Oxidative stress and metabolic pathologies: from an adipocentric point of view. Oxid. Med. Cell. Longev. 2014, 908539.

https://doi.org/10.1155/2014/908539

Le Port, A., Gueguen, A., Kesse-Guyot, E., Melchior, M., Lemogne, C., Nabi, H., Goldberg, M., Zins, M., Czernichow, S., 2012. Association between Dietary Patterns and Depressive Symptoms Over Time: A 10-Year FollowUp Study of the GAZEL Cohort. PLoS One 7, e51593. https://doi.org/10.1371/journal.pone.0051593

Lee, B.-C., Lee, J., 2014. Cellular and molecular players in adipose tissue inflammation in the development of obesity-induced insulin resistance. Biochim. Biophys. Acta 1842, 446-62.

https://doi.org/10.1016/j.bbadis.2013.05.017

Lee, J., Ellis, J.M., Wolfgang, M.J., 2015. Adipose fatty acid oxidation is 
required for thermogenesis and potentiates oxidative stress-induced inflammation. Cell Rep. 10, 266-79.

https://doi.org/10.1016/j.celrep.2014.12.023

Li, C.-T., Su, T.-P., Wang, S.-J., Tu, P.-C., Hsieh, J.-C., 2015. Prefrontal glucose metabolism in medication-resistant major depression. Br. J. Psychiatry 206, 316-23. https://doi.org/10.1192/bjp.bp.113.140434

Li, J., Yang, Y.L., Li, L.Z., Zhang, L., Liu, Q., Liu, K., Li, P., Liu, B., Qi, L.W., 2017. Succinate accumulation impairs cardiac pyruvate dehydrogenase activity through GRP91-dependent and independent signaling pathways: Therapeutic effects of ginsenoside Rb1. Biochim. Biophys. Acta - Mol. Basis Dis. 1863, 2835-2847. https://doi.org/10.1016/j.bbadis.2017.07.017

Li, M., Fallin, M.D., Riley, A., Landa, R., Walker, S.O., Silverstein, M., Caruso, D., Pearson, C., Kiang, S., Dahm, J.L., Hong, X., Wang, G., Wang, M.-C., Zuckerman, B., Wang, X., 2016. The Association of Maternal Obesity and Diabetes With Autism and Other Developmental Disabilities. Pediatrics 137, e20152206. https://doi.org/10.1542/peds.2015-2206

Li, Y.-M., Shen, Y.-D., Li, Y.-J., Xun, G.-L., Liu, H., Wu, R.-R., Xia, K., Zhao, J.-P., Ou, J.-J., 2018. Maternal dietary patterns, supplements intake and autism spectrum disorders: A preliminary case-control study. Medicine (Baltimore). 97, e13902. https://doi.org/10.1097/MD.0000000000013902

Link, J.C., Chen, X., Prien, C., Borja, M.S., Hammerson, B., Oda, M.N., Arnold, A.P., Reue, K., 2015. Increased High-Density Lipoprotein Cholesterol Levels in Mice With XX Versus XY Sex Chromosomes. Arterioscler. Thromb. Vasc. Biol. 35, 1778-1786. https://doi.org/10.1161/ATVBAHA.115.305460

Liu, T., Zhang, L., Joo, D., Sun, S.-C., 2017. NF- $x$ B signaling in inflammation. Signal Transduct. Target. Ther. 2.

https://doi.org/10.1038/sigtrans.2017.23

Lumeng, C.N., Saltiel, A.R., 2011. Inflammatory links between obesity and metabolic disease. J. Clin. Invest. 121, 2111-7.

https://doi.org/10.1172/JCI57132

Lyall, K., Munger, K.L., O'Reilly, É.J., Santangelo, S.L., Ascherio, A., 2013. Maternal dietary fat intake in association with autism spectrum disorders. Am. J. Epidemiol. 178, 209-20.

https://doi.org/10.1093/aje/kws433

Lyra E Silva, N. de M., Lam, M.P., Soares, C.N., Munoz, D.P., Milev, R., De Felice, F.G., 2019. Insulin Resistance as a Shared Pathogenic Mechanism Between Depression and Type 2 Diabetes. Front. psychiatry 10, 57. https://doi.org/10.3389/fpsyt.2019.00057 
MacKellar, J., Cushman, S.W., Periwal, V., 2010. Waves of adipose tissue growth in the genetically obese Zucker fatty rat. PLoS One 5, e8197. https://doi.org/10.1371/journal.pone.0008197

Mahmood, S., Kida, T., Izumi, A., Sasaki, C., Okamoto, H., Kobayashi, H., Yamada, G., 2008. Assessment of Health Related Quality of Life in Chronic Liver Disease Patients Using the Japanese Versions of CLDQ and SF-36. Open Gastroenterol. J. 2, 57-63. https://doi.org/10.2174/1874259900802010057

Maina, G., Salvi, V., Vitalucci, A., D'Ambrosio, V., Bogetto, F., 2008. Prevalence and correlates of overweight in drug-naïve patients with bipolar disorder. J. Affect. Disord. 110, 149-155. https://doi.org/10.1016/j.jad.2007.12.233

Malik, V.S., Popkin, B.M., Bray, G.A., Despres, J.-P., Willett, W.C., Hu, F.B., 2010. Sugar-Sweetened Beverages and Risk of Metabolic Syndrome and Type 2 Diabetes: A meta-analysis. Diabetes Care 33, 2477-2483. https://doi.org/10.2337/dc10-1079

Margariti, E., Deutsch, M., Manolakopoulos, S., Papatheodoridis, G. V, 2012. Non-alcoholic fatty liver disease may develop in individuals with normal body mass index. Ann. Gastroenterol. 25, 45-51. https://doi.org/24713801

Margoob, M.A., Mushtaq, D., 2011. Serotonin transporter gene polymorphism and psychiatric disorders: is there a link? Indian J. Psychiatry 53, 289-99. https://doi.org/10.4103/0019-5545.91901

Matute, C., Domercq, M., Sánchez-Gómez, M.-V., 2006. Glutamatemediated glial injury: mechanisms and clinical importance. Glia 53, 212-24. https://doi.org/10.1002/glia.20275

Maugeri, A., Barchitta, M., 2019. A Systematic Review of Ecological Momentary Assessment of Diet: Implications and Perspectives for Nutritional Epidemiology. Nutrients 11. https://doi.org/10.3390/nu11112696

McCauley, J.L., Olson, L.M., Dowd, M., Amin, T., Steele, A., Blakely, R.D., Folstein, S.E., Haines, J.L., Sutcliffe, J.S., 2004. Linkage and association analysis at the serotonin transporter (SLC6A4) locus in a rigidcompulsive subset of autism. Am. J. Med. Genet. 127B, 104-112. https://doi.org/10.1002/ajmg.b.20151

Mielke, J.G., Nicolitch, K., Avellaneda, V., Earlam, K., Ahuja, T., Mealing, G., Messier, C., 2006. Longitudinal study of the effects of a high-fat diet on glucose regulation, hippocampal function, and cerebral insulin sensitivity in C57BL/6 mice. Behav. Brain Res. 175, 374-382. https://doi.org/10.1016/j.bbr.2006.09.010 
Miller, A.H., Haroon, E., Raison, C.L., Felger, J.C., 2013. Cytokine targets in the brain: impact on neurotransmitters and neurocircuits. Depress. Anxiety 30, 297-306. https://doi.org/10.1002/da.22084

Millichap, J.G., Yee, M.M., 2012. The Diet Factor in AttentionDeficit/Hyperactivity Disorder. Pediatrics 129, 330-337. https://doi.org/10.1542/peds.2011-2199

Minamino, T., Orimo, M., Shimizu, I., Kunieda, T., Yokoyama, M., Ito, T., Nojima, A., Nabetani, A., Oike, Y., Matsubara, H., Ishikawa, F., Komuro, I., 2009. A crucial role for adipose tissue p53 in the regulation of insulin resistance. Nat. Med. 15, 1082-7. https://doi.org/10.1038/nm.2014

Minihane, A.M., Vinoy, S., Russell, W.R., Baka, A., Roche, H.M., Tuohy, K.M., Teeling, J.L., Blaak, E.E., Fenech, M., Vauzour, D., McArdle, H.J., Kremer, B.H.A., Sterkman, L., Vafeiadou, K., Benedetti, M.M., Williams, C.M., Calder, P.C., 2015. Low-grade inflammation, diet composition and health: current research evidence and its translation. Br. J. Nutr. 114, 9991012. https://doi.org/10.1017/S0007114515002093

Mitelman, S.A., Bralet, M.-C., Mehmet Haznedar, M., Hollander, E., Shihabuddin, L., Hazlett, E.A., Buchsbaum, M.S., 2018. Positron emission tomography assessment of cerebral glucose metabolic rates in autism spectrum disorder and schizophrenia. Brain Imaging Behav. 12, 532-546. https://doi.org/10.1007/s11682-017-9721-z

Morón, J.A., Zakharova, I., Ferrer, J. V, Merrill, G.A., Hope, B., Lafer, E.M., Lin, Z.C., Wang, J.B., Javitch, J.A., Galli, A., Shippenberg, T.S., 2003. Mitogen-activated protein kinase regulates dopamine transporter surface expression and dopamine transport capacity. J. Neurosci. 23, 8480-8.

Murray, A.J., Knight, N.S., Cochlin, L.E., McAleese, S., Deacon, R.M.J., Rawlins, J.N.P., Clarke, K., 2009. Deterioration of physical performance and cognitive function in rats with short-term high-fat feeding. FASEB J. 23, 4353-4360. https://doi.org/10.1096/fj.09-139691

Na, W., Chung, B., Sohn, C., 2019. A Relationship between Dietary Patterns and Dyslipidemia in Urban-dwelling Middle-Aged Korean Men: Using Korean Genome and Epidemiology Study (KoGES). Clin. Nutr. Res. 8, 219. https://doi.org/10.7762/cnr.2019.8.3.219

Nguyen, J.C.D., Killcross, A.S., Jenkins, T.A., 2014. Obesity and cognitive decline: role of inflammation and vascular changes. Front. Neurosci. 8, 375. https://doi.org/10.3389/fnins.2014.00375

O’Neil, A., Quirk, S.E., Housden, S., Brennan, S.L., Williams, L.J., Pasco, J.A., Berk, M., Jacka, F.N., 2014. Relationship Between Diet and Mental 
Health in Children and Adolescents: A Systematic Review. Am. J. Public Health 104, e31-e42. https://doi.org/10.2105/AJPH.2014.302110

Oddy, W.H., Allen, K.L., Trapp, G.S.A., Ambrosini, G.L., Black, L.J., Huang, R.-C., Rzehak, P., Runions, K.C., Pan, F., Beilin, L.J., Mori, T.A., 2018. Dietary patterns, body mass index and inflammation: Pathways to depression and mental health problems in adolescents. Brain. Behav. Immun. 69, 428-439. https://doi.org/10.1016/j.bbi.2018.01.002

Osburg, B., Peiser, C., Dömling, D., Schomburg, L., Ko, Y.T., Voigt, K., Bickel, U., 2002. Effect of endotoxin on expression of TNF receptors and transport of TNF-alpha at the blood-brain barrier of the rat. Am. J. Physiol. Endocrinol. Metab. 283, E899-908.

https://doi.org/10.1152/ajpendo.00436.2001

Pagoto, S.L., Curtin, C., Lemon, S.C., Bandini, L.G., Schneider, K.L., Bodenlos, J.S., Ma, Y., 2009. Association Between Adult Attention Deficit/Hyperactivity Disorder and Obesity in the US Population. Obesity 17, 539-544. https://doi.org/10.1038/oby.2008.587

Pan, X.-R., Li, G.-W., Hu, Y.-H., Wang, J.-X., Yang, W.-Y., An, Z.-X., Hu, Z.-X., Juan-Lin, Xiao, J.-Z., Cao, H.-B., Liu, P.-A., Jiang, X.-G., Jiang, Y.-Y., Wang, J.-P., Zheng, H., Zhang, H., Bennett, P.H., Howard, B. V., 1997. Effects of Diet and Exercise in Preventing NIDDM in People With Impaired Glucose Tolerance: The Da Qing IGT and Diabetes Study. Diabetes Care 20, 537-544. https://doi.org/10.2337/diacare.20.4.537

Papazoglou, I.K., Jean, A., Gertler, A., Taouis, M., Vacher, C.-M., 2015. Hippocampal GSK3 $\beta$ as a Molecular Link Between Obesity and Depression. Mol. Neurobiol. 52, 363-74. https://doi.org/10.1007/s12035-014-8863-x

Park, J., Choe, S.S., Choi, A.H., Kim, K.H., Yoon, M.J., Suganami, T., Ogawa, Y., Kim, J.B., 2006. Increase in glucose-6-phosphate dehydrogenase in adipocytes stimulates oxidative stress and inflammatory signals. Diabetes 55, 2939-49. https://doi.org/10.2337/db05-1570

Pomytkin, I., Costa-Nunes, J.P., Kasatkin, V., Veniaminova, E., Demchenko, A., Lyundup, A., Lesch, K.-P., Ponomarev, E.D., Strekalova, T., 2018. Insulin receptor in the brain: Mechanisms of activation and the role in the CNS pathology and treatment. CNS Neurosci. Ther. https://doi.org/10.1111/cns.12866

Pomytkin, I.A., Cline, B.H., Anthony, D.C., Steinbusch, H.W., Lesch, K.P., Strekalova, T., 2015. Endotoxaemia resulting from decreased serotonin tranporter (5-HTT) function: a reciprocal risk factor for depression and insulin resistance? Behav. Brain Res. 276, 111-7. 
https://doi.org/10.1016/j.bbr.2014.04.049

Psaltopoulou, T., Sergentanis, T.N., Panagiotakos, D.B., Sergentanis, I.N., Kosti, R., Scarmeas, N., 2013. Mediterranean diet, stroke, cognitive impairment, and depression: A meta-analysis. Ann. Neurol. 74, 580-591. https://doi.org/10.1002/ana.23944

Racké, K., Reimann, A., Schwörer, H., Kilbinger, H., 1996. Regulation of 5-HT release from enterochromaffin cells. Behav. Brain Res. 73, 83-7. https://doi.org/10.1016/0166-4328(96)00075-7

Rahman, I., Gilmour, P.S., Jimenez, L.A., MacNee, W., 2002. Oxidative stress and TNF-alpha induce histone acetylation and NF-kappaB/AP-1 activation in alveolar epithelial cells: potential mechanism in gene transcription in lung inflammation. Mol. Cell. Biochem. 234-235, 239-48.

Reichelt, A.C., Gibson, G.D., Abbott, K.N., Hare, D.J., 2019. A high-fat high-sugar diet in adolescent rats impairs social memory and alters chemical markers characteristic of atypical neuroplasticity and parvalbumin interneuron depletion in the medial prefrontal cortex. Food Funct. 10, 19851998. https://doi.org/10.1039/c8fo02118j

Reynolds, C.F., Thomas, S.B., Morse, J.Q., Anderson, S.J., Albert, S., Dew, M.A., Begley, A., Karp, J.F., Gildengers, A., Butters, M.A., Stack, J.A., Kasckow, J., Miller, M.D., Quinn, S.C., 2014. Early intervention to preempt major depression among older black and white adults. Psychiatr. Serv. 65, 765-73. https://doi.org/10.1176/appi.ps.201300216

Rippe, J.M., Angelopoulos, T.J., 2015. Fructose-Containing Sugars and Cardiovascular Disease. Adv. Nutr. 6, 430-439.

https://doi.org/10.3945/an.114.008177

Ruhl, C.E., Everhart, J.E., 2004. Epidemiology of nonalcoholic fatty liver. Clin. Liver Dis. 8, 501-519. https://doi.org/10.1016/j.cld.2004.04.008

Sadakane, A., Tsutsumi, A., Gotoh, T., Ishikawa, S., Ojima, T., Kario, K., Nakamura, Y., Kayaba, K., 2008. Dietary patterns and levels of blood pressure and serum lipids in a Japanese population. J. Epidemiol. 18, 58-67. https://doi.org/10.2188/jea.18.58

Salinero, A.E., Anderson, B.M., Zuloaga, K.L., 2018. Sex differences in the metabolic effects of diet-induced obesity vary by age of onset. Int. J. Obes. 42, 1088-1091. https://doi.org/10.1038/s41366-018-0023-3

Salmerón, J., 1997. Dietary Fiber, Glycemic Load, and Risk of Noninsulin-dependent Diabetes Mellitus in Women. JAMA J. Am. Med. Assoc. 277, 472. https://doi.org/10.1001/jama.1997.03540300040031

Salmeron, J., Ascherio, A., Rimm, E.B., Colditz, G.A., Spiegelman, D., 
Jenkins, D.J., Stampfer, M.J., Wing, A.L., Willett, W.C., 1997. Dietary Fiber, Glycemic Load, and Risk of NIDDM in Men. Diabetes Care 20, 545550. https://doi.org/10.2337/diacare.20.4.545

Sami, W., Ansari, T., Butt, N.S., Hamid, M.R.A., 2017. Effect of diet on type 2 diabetes mellitus: A review. Int. J. Health Sci. (Qassim). 11, 65-71.

Sánchez-Villegas, A., Toledo, E., de Irala, J., Ruiz-Canela, M., Pla-Vidal, J., Martínez-González, M.A., 2012. Fast-food and commercial baked goods consumption and the risk of depression. Public Health Nutr. 15, 424-432. https://doi.org/10.1017/S1368980011001856

Sanmiguel, C., Gupta, A., Mayer, E.A., 2015. Gut Microbiome and Obesity: A Plausible Explanation for Obesity. Curr. Obes. Rep. 4, 250-61. https://doi.org/10.1007/s13679-015-0152-0

Sarris, J., Logan, A.C., Akbaraly, T.N., Amminger, G.P., BalanzáMartínez, V., Freeman, M.P., Hibbeln, J., Matsuoka, Y., Mischoulon, D., Mizoue, T., Nanri, A., Nishi, D., Ramsey, D., Rucklidge, J.J., SanchezVillegas, A., Scholey, A., Su, K.-P., Jacka, F.N., 2015. Nutritional medicine as mainstream in psychiatry. The Lancet Psychiatry 2, 271-274. https://doi.org/10.1016/S2215-0366(14)00051-0

Schulze, M.B., 2004. Sugar-Sweetened Beverages, Weight Gain, and Incidence of Type 2 Diabetes in Young and Middle-Aged Women. JAMA 292, 927. https://doi.org/10.1001/jama.292.8.927

Schweiger, U., Greggersen, W., Rudolf, S., Pusch, M., Menzel, T., Winn, S., Hassfurth, J., Fassbinder, E., Kahl, K.G., Oltmanns, K.M., Hohagen, F., Peters, A., 2008. Disturbed Glucose Disposal in Patients With Major Depression; Application of the Glucose Clamp Technique. Psychosom. Med. 70, 170-176. https://doi.org/10.1097/PSY.0b013e318164231d

Shedlock, K., Susi, A., Gorman, G.H., Hisle-Gorman, E., Erdie-Lalena, C.R., Nylund, C.M., 2016. Autism Spectrum Disorders and Metabolic Complications of Obesity. J. Pediatr. 178, 183-187.e1. https://doi.org/10.1016/j.jpeds.2016.07.055

Sparks, D.L., 2008. The early and ongoing experience with the cholesterolfed rabbit as a model of Alzheimer's disease: the old, the new and the pilot. J. Alzheimers. Dis. 15, 641-56.

Stojakovic, A., Mastronardi, C.A., Licinio, J., Wong, M.-L., 2018. Longterm consumption of high-fat diet impairs motor coordination without affecting the general motor activity. J. Transl. Sci. 5, 1-10.

https://doi.org/10.15761/JTS.1000295

Strekalova, T., Costa-Nunes, J.P., Veniaminova, E., Kubatiev, A., Lesch, 
K.-P., Chekhonin, V.P., Evans, M.C., Steinbusch, H.W.M., 2016. Insulin receptor sensitizer, dicholine succinate, prevents both Toll-like receptor 4 (TLR4) upregulation and affective changes induced by a high-cholesterol diet in mice. J. Affect. Disord. 196, 109-116.

https://doi.org/10.1016/j.jad.2016.02.045

Strekalova, T., Evans, M., Costa-Nunes, J., Bachurin, S., Yeritsyan, N., Couch, Y., Steinbusch, H.M.W., Köhler, S.E., Lesch, K.-P., Anthony, D.C., 2015. Tr4 upregulation in the brain accompanies depression- and anxietylike behaviors induced by a high-cholesterol diet. Brain. Behav. Immun. 48 . https://doi.org/10.1016/j.bbi.2015.02.015

Sung, F.L., Zhu, T.Y., Au-Yeung, K.K.W., Siow, Y.L., O, K., 2002. Enhanced MCP-1 expression during ischemia/reperfusion injury is mediated by oxidative stress and NF-kappaB. Kidney Int. 62, 1160-70. https://doi.org/10.1111/j.1523-1755.2002.kid577.x

Suren, P., Gunnes, N., Roth, C., Bresnahan, M., Hornig, M., Hirtz, D., Lie, K.K., Lipkin, W.I., Magnus, P., Reichborn-Kjennerud, T., Schjolberg, S., Susser, E., Oyen, A.-S., Smith, G.D., Stoltenberg, C., 2014. Parental Obesity and Risk of Autism Spectrum Disorder. Pediatrics 133, e1128-e1138. https://doi.org/10.1542/peds.2013-3664

Sutcliffe, J.S., Delahanty, R.J., Prasad, H.C., McCauley, J.L., Han, Q., Jiang, L., Li, C., Folstein, S.E., Blakely, R.D., 2005. Allelic Heterogeneity at the Serotonin Transporter Locus (SLC6A4) Confers Susceptibility to Autism and Rigid-Compulsive Behaviors. Am. J. Hum. Genet. 77, 265-279.

https://doi.org/10.1086/432648

Taurines, R., Schwenck, C., Westerwald, E., Sachse, M., Siniatchkin, M., Freitag, C., 2012. ADHD and autism: differential diagnosis or overlapping traits? A selective review. ADHD Atten. Deficit Hyperact. Disord. 4, 115139. https://doi.org/10.1007/s12402-012-0086-2

Tilg, H., Moschen, A.R., 2006. Adipocytokines: mediators linking adipose tissue, inflammation and immunity. Nat. Rev. Immunol. 6, 772-783.

https://doi.org/10.1038/nri1937

Tomeno, W., Kawashima, K., Yoneda, M., Saito, S., Ogawa, Y., Honda, Y., Kessoku, T., Imajo, K., Mawatari, H., Fujita, K., Saito, S., Hirayasu, Y., Nakajima, A., 2015. Non-alcoholic fatty liver disease comorbid with major depressive disorder: The pathological features and poor therapeutic efficacy. J. Gastroenterol. Hepatol. 30, 1009-1014. https://doi.org/10.1111/jgh.12897

Tordjman, S., Gutknecht, L., Carlier, M., Spitz, E., Antoine, C., Slama, F., Carsalade, V., Cohen, D.J., Ferrari, P., Roubertoux, P.L., Anderson, 
G.M., 2001. Role of the serotonin transporter gene in the behavioral expression of autism. Mol. Psychiatry 6, 434-439. https://doi.org/10.1038/sj.mp.4000873

Turnbaugh, P.J., Bäckhed, F., Fulton, L., Gordon, J.I., 2008. Diet-induced obesity is linked to marked but reversible alterations in the mouse distal gut microbiome. Cell Host Microbe 3, 213-23.

https://doi.org/10.1016/j.chom.2008.02.015

Vagena, E., Ryu, J.K., Baeza-Raja, B., Walsh, N.M., Syme, C., Day, J.P., Houslay, M.D., Baillie, G.S., 2019. A high-fat diet promotes depression-like behavior in mice by suppressing hypothalamic PKA signaling. Transl. Psychiatry 9, 141. https://doi.org/10.1038/s41398-019-0470-1

van Dam, R.M., Grievink, L., Ocké, M.C., Feskens, E.J., 2003. Patterns of food consumption and risk factors for cardiovascular disease in the general Dutch population. Am. J. Clin. Nutr. 77, 1156-1163.

https://doi.org/10.1093/ajcn/77.5.1156

van Dam, R.M., Rimm, E.B., Willett, W.C., Stampfer, M.J., Hu, F.B., 2002. Dietary Patterns and Risk for Type 2 Diabetes Mellitus in U.S. Men. Ann. Intern. Med. 136, 201. https://doi.org/10.7326/0003-4819-136-3200202050-00008

Vanicek, T., Kutzelnigg, A., Philippe, C., Sigurdardottir, H.L., James, G.M., Hahn, A., Kranz, G.S., Höflich, A., Kautzky, A., Traub-Weidinger, T., Hacker, M., Wadsak, W., Mitterhauser, M., Kasper, S., Lanzenberger, R., 2017. Altered interregional molecular associations of the serotonin transporter in attention deficit/hyperactivity disorder assessed with PET. Hum. Brain Mapp. 38, 792-802. https://doi.org/10.1002/hbm.23418

Wang, H., Sun, J., Goldstein, H., 2008. Human immunodeficiency virus type 1 infection increases the in vivo capacity of peripheral monocytes to cross the blood-brain barrier into the brain and the in vivo sensitivity of the blood-brain barrier to disruption by lipopolysaccharide. J. Virol. 82, 7591600. https://doi.org/10.1128/JVI.00768-08

Webber, L., Divajeva, D., Marsh, T., McPherson, K., Brown, M., Galea, G., Breda, J., 2014. The future burden of obesity-related diseases in the 53 WHO European-Region countries and the impact of effective interventions: a modelling study. BMJ Open 4, e004787-e004787.

https://doi.org/10.1136/bmjopen-2014-004787

Weinberg, W.A., Brumback, R.A., 1990. Primary disorder of vigilance: A novel explanation of inattentiveness, daydreaming, boredom, restlessness, and sleepiness. J. Pediatr. 116, 720-725. https://doi.org/10.1016/S0022- 
3476(05)82654-X

Weinstein, A.A., Kallman Price, J., Stepanova, M., Poms, L.W., Fang, Y., Moon, J., Nader, F., Younossi, Z.M., 2011. Depression in Patients with Nonalcoholic Fatty Liver Disease and Chronic Viral Hepatitis B and C. Psychosomatics 52, 127-132. https://doi.org/10.1016/j.psym.2010.12.019

Wolburg, H., Lippoldt, A., 2002. Tight junctions of the blood-brain barrier: development, composition and regulation. Vascul. Pharmacol. 38, $323-37$.

Woods, S., Seeley, R., Baskin, D., Schwartz, M., 2003. Insulin and the Blood-Brain Barrier. Curr. Pharm. Des. 9, 795-800.

https://doi.org/10.2174/1381612033455323

Yamada, N., Katsuura, G., Ochi, Y., Ebihara, K., Kusakabe, T., Hosoda, K., Nakao, K., 2011. Impaired CNS leptin action is implicated in depression associated with obesity. Endocrinology 152, 2634-43. https://doi.org/10.1210/en.2011-0004

Yamada, T., Inui, A., Hayashi, N., Fujimura, M., Fujimiya, M., 2003. Serotonin stimulates endotoxin translocation via 5 -HT3 receptors in the rat ileum. Am. J. Physiol. Gastrointest. Liver Physiol. 284, G782-8. https://doi.org/10.1152/ajpgi.00376.2002

Yang, W.Y., Williams, L.T., Collins, C., Siew Swee, C.W., 2012. The relationship between dietary patterns and overweight and obesity in children of Asian developing countries: A Systematic Review. JBI Database Syst. Rev. Implement. Reports 10, 4568-4599. https://doi.org/10.11124/jbisrir-2012407

Yirmiya, N., Pilowsky, T., Nemanov, L., Arbelle, S., Feinsilver, T., Fried, I., Ebstein, R.P., 2001. Evidence for an association with the serotonin transporter promoter region polymorphism and autism. Am. J. Med. Genet. 105, 381-386. https://doi.org/10.1002/ajmg. 1365

Yiu, J.H.C., Dorweiler, B., Woo, C.W., 2017. Interaction between gut microbiota and toll-like receptor: from immunity to metabolism. J. Mol. Med. (Berl). 95, 13-20. https://doi.org/10.1007/s00109-016-1474-4

Youssef, N.A., Abdelmalek, M.F., Binks, M., Guy, C.D., Omenetti, A., Smith, A.D., Diehl, A.M.E., Suzuki, A., 2013. Associations of depression, anxiety and antidepressants with histological severity of nonalcoholic fatty liver disease. Liver Int. 33, 1062-1070. https://doi.org/10.1111/liv.12165

Yuzefovych, L. V, Musiyenko, S.I., Wilson, G.L., Rachek, L.I., 2013. Mitochondrial DNA damage and dysfunction, and oxidative stress are associated with endoplasmic reticulum stress, protein degradation and 
apoptosis in high fat diet-induced insulin resistance mice. PLoS One 8, e54059. https://doi.org/10.1371/journal.pone.0054059

Zametkin, A.J., Nordahl, T.E., Gross, M., King, A.C., Semple, W.E., Rumsey, J., Hamburger, S., Cohen, R.M., 1990. Cerebral Glucose Metabolism in Adults with Hyperactivity of Childhood Onset. N. Engl. J. Med. 323, 13611366. https://doi.org/10.1056/NEJM199011153232001

Zhang, H., Sun, S.-C., 2015. NF- $\varkappa$ B in inflammation and renal diseases. Cell Biosci. 5, 63. https://doi.org/10.1186/s13578-015-0056-4 


\section{Chapter 2}

\section{Autism-like behaviours and memory deficits result from a Western diet in mice}




\section{Chapter 2. Autism-like behaviours and memory deficits result from a Western diet in mice}

Ekaterina Veniaminova ${ }^{1,2}$, Raymond Cespuglio ${ }^{3}$, Chi Wai Cheung ${ }^{4}$, Alexei Umriukhin ${ }^{2}$, Nataliia Markova ${ }^{1,2,6}$, Elena Shevtsova ${ }^{6}$, Klaus-Peter Lesch ${ }^{1,2,7}$, Daniel C. Anthony ${ }^{8}$, and Tatyana Strekalova ${ }^{1,2,7}$

${ }^{1}$ Department of Neuroscience, School for Mental Health and Neuroscience, Maastricht University, Universiteitssingel 40, 6200 MD Maastricht, Netherlands; ${ }^{2}$ Institute of Molecular Medicine, Sechenov First Moscow State Medical University, Moscow 119991, Russia; ${ }^{3}$ Faculty of Medicine, Neuroscience Research Center of Lyon, C. Bernard University, 8 Av. Rockefeller, 69373 Lyon, France; ${ }^{4}$ Department of Anaesthesiology, Queen Mary Hospital, The University of Hong Kong, 102 Pokfulam, Hong Kong; ${ }^{5}$ Department of Normal Physiology, Sechenov First Moscow State Medical University, Moscow 119991, Russia; ${ }^{6}$ Laboratory of Biomolecular Screening, Institute of Physiologically Active Compounds, Russian Academy of Sciences, Moscow Region, Russia; ${ }^{7}$ Division of Molecular Psychiatry, Laboratory of Translational Neuroscience, Department of Psychiatry, Psychosomatics and Psychotherapy, University of Würzburg, Fuechsleinstrasse 15, 97080 Würzburg, Germany; ${ }^{8}$ Department of Pharmacology, Oxford University, Mansfield Road, Oxford OX1 3QT, UK

Veniaminova E, Cespuglio R, Cheung CW, Umriukhin A, Markova N, Shevtsova E, Lesch KP, Anthony DC, Strekalova T. Autism-Like Behaviours and Memory Deficits Result from a Western Diet in Mice. Neural Plasticity. 2017;2017:9498247. 


\section{Abstract}

Nonalcoholic fatty liver disease, induced by a Western diet (WD), evokes central and peripheral inflammation that is accompanied by altered emotionality. These changes can be associated with abnormalities in social behaviour, hippocampus-dependent cognitive functions, and metabolism. Female C57BL/6J mice were fed with a regular chow or with a WD containing $0.2 \%$ of cholesterol and $21 \%$ of saturated fat for three weeks. WD-treated mice exhibited increased social avoidance, crawl-over and digging behaviours, decreased bodybody contacts, and hyperlocomotion. The WD-fed group also displayed deficits in hippocampal-dependent performance such as contextual memory in a fear conditioning and pellet displacement paradigms. A reduction in glucose tolerance and elevated levels of serum cholesterol and leptin were also associated with the WD. The peroxisome proliferator-activated receptor gamma coactivator 1-alpha (PPARGC1a) mRNA, a marker of mitochondrial activity, was decreased in the prefrontal cortex, hippocampus, hypothalamus, and dorsal raphe, suggesting suppressed brain mitochondrial functions, but not in the liver. This is the first report to show that a WD can profoundly suppress social interactions and induce dominant-like behaviours in naïve adult mice. The spectrum of behaviours that were found to be induced are reminiscent of symptoms associated with autism, and, if paralleled in humans, suggest that a WD might exacerbate autism spectrum disorder. 


\section{Introduction}

In the context of increasing societal preference for "cafeteria-type diets" and "comfortable food" enriched with unsaturated fat and sugars, as well as the so-called "Western-type diet", which is predominantly based on a heightened intake of saturated fat and cholesterol, there is an urgent need to study the physiological consequences of these diets. Excessive consumption of the Western diet has been shown to generate obesity, insulin resistance, hypercholesterolemia, and neuroinflammation in many organs, including the brain [1-6]. In addition, a Western-type diet may impact on reciprocal cognition $[5,7]$ and social interactions [3]. Human and animal studies suggest important roles of increased fat/cholesterol-containing dietary regimen in behavioural abnormalities associated with social behaviour, aggression, and brain plasticity [7-12].

Recent experiments showed aberrant social interactions and increased measures of aggression associated with diets containing high amounts of fat and cholesterol. Dietary exposure to increased amounts of cholesterol and fat was shown to increase male aggression in monkeys [13], elevate the risk of autism spectrum disorder in offspring of humans approximately 1.5 times [14], and suppress social exploratory interactions in offspring of mice $[15,16]$. Also, a combination of elevated contents of cholesterol and saturated fat was found to aggravate cognitive rigidity and social deficiency in the mouse 
model of autism [3]. Yet, these and other recently reported results provide limited information regarding the effects of a "Western"/westernized diet on social behaviours of adult individuals not predisposed to any abnormalities, including the changes typical for autism-like spectrum disorder.

The mechanisms that underlie deficient social interactions and brain plasticity associated with westernized diets can overlap. Increased rates of aggression-like traits are suggested to be related to reduced cognitive control and deficiencies in cognitive functions in general $[17,18]$. Most of available literature reports decreased learning abilities in animals exposed to high-fat and high-cholesterol diets. Housing of rats on a high-cholesterol and fat-containing diet containing $2 \%$ cholesterol for 4 months led to impaired learning in the Morris water maze [19]; a similar diet induced altered stress response [20]. Spatial learning in an 8-arm maze was shown to be impaired in mice and rats housed on high-cholesterol and fat-containing diets $[9,12$, 21, 22]. A high-fat diet containing $2 \%$ cholesterol induced deficits in contextual conditioning that were accompanied by altered hippocampal structure, such as a reduction in microtubule-associated proteins and elevation of markers of microglial activation in a rat [21]. Yet, very few studies addressing the effects of a westernized-type diet in the fear conditioning paradigms have been reported so far, while this paradigm provides higher sensitivity than other models to explore the 
integrity of hippocampal functions [23]. In addition, a high-fat/highcholesterol diet was also associated with increased immobility in the forced swim test indicating that the diet also generates a negative affect [21].

Several metabolic endpoints in the high-fat/high-cholesterol dietfed mice have been noted that may underpin the behavioural changes, and these include increased insulin resistance, which is associated with a reduction in the expression of peroxisome proliferator-activated receptor gamma coactivator 1a (PPARGC1a), a marker of mitochondrial disbalance and impaired mitochondrial activity in dietaryinduced type 2 diabetes [16, 24-26]. Diminished expression of this gene has been reported in the human hippocampus in patients with Alzheimer's disorder, and its expression level correlates with the clinical progression of dementia [27]. Mice genetically lacking the PPARGC1a gene exhibit an imbalance between inhibitory and excitatory synaptic transmission in the hippocampus, a mechanism that is suggested to be an important pathogenetic factor of autism [28, 29]. Thus, cognitive and other behavioural deficits reported in this work on mice fed with high-fat/high-cholesterol diet can result not only from lowered mitochondrial activity that is associated with decreased PPARGC1a brain levels but also from the specific role of this molecule in synaptic plasticity. 
Our previous studies in mice employing a high-fat/highcholesterol diet to induce nonalcoholic fatty liver disease (NAFLD) revealed that the diet increased levels of impulsivity, behavioural despair, anxiety, and reduced exploration of novel objects [30-32]. However, to date, the effects of excessive dietary cholesterol on social behaviours and aggression were not studied in this model. Here, we exposed female C57BL/6J mice to the Western diet (WD) $[30,31]$ to investigate the impact of the diet on social interactions, hippocampal function, and metabolism in normal mice exposed to the westernized diet during their adulthood. We are able to report, for the first time, that experimental exposure of mice to the WD profoundly affects their social interactions, substantially suppressing social exploratory contacts, inducing dominant-like behaviours and aberrant patterns of social behaviour. This diet also caused a deficient hippocampusdependent performance, increased locomotion, downregulated mitochondrial activity marker PPARGC1a in the brain but not in the liver, and induced glucose intolerance and hyperleptin / hypercholesterolemia in mice. Thus, under conditions of excessive intake of fat and cholesterol which do not alter gross physiological measures such as body weight, substantial changes in behaviour, some of which are reminiscent of symptoms associated with autism, can occur. 


\section{Materials and methods}

\subsection{Animals}

Studies were performed using 3-month-old female C57BL/6J from Janvier, Charles River, France. Mice were housed five per cage during the study, under a $12 \mathrm{~h}$ light-dark cycle (lights on: 19:00 h) with food and water ad libitum and under controllable laboratory conditions $\left(22 \pm 1^{\circ} \mathrm{C}, 55 \%\right.$ humidity). All experiments were carried out in accordance with the European Communities Council Directive for the care and use of laboratory animals (2010/63/EU) upon approval by the Ethical Committee of the C. Bernard University on animal care and welfare.

\subsection{Study design and dietary challenge}

Mice were fed with a regular laboratory diet with an energy content of $3.0 \mathrm{kcal} / \mathrm{g}, 6.55 \%$ unsaturated fat, and $65 \%$ carbohydrates (Mucedola s.r.l., Settimo, Italy) or with a diet containing $0.2 \%(\mathrm{w} / \mathrm{w})$ cholesterol, $20 \%$ of saturated fat, $39 \%$ carbohydrates, and an energy content of $4.6 \mathrm{kcal} / \mathrm{g}$, "Western diet" (WD), (Research Diet Inc., New Brunswick, NJ, USA) for three weeks as described elsewhere [30-32]. The content of the nutrients in calories and weight is indicated in Figure 1(a) and Supplementary Table 1 available online at https://doi.org/10.1155/2017/9498247. Mice from the two groups were compared for the parameters of social interaction, hippocampusdependent performance, and several metabolic read-outs, in three 
separate experiments. Body weight and food intake were monitored weekly in each study, as described elsewhere (Figures 1(b) and 1(c); [30-32]) and were calculated as normalized to the mean of a control group.

After a three-week period of dietary challenge, a cohort of mice was studied for social interactions in a home cage and in a food competition test, as well as for an acquisition and an extinction of contextual fear in the fear conditioning paradigm (study $1 ; \mathrm{n}=14$ for the control group and $n=20$ for the group fed with a high-cholesterol and fat-containing diet; Figure 1(d)). Another cohort of animals was tested in the pellet displacement tube test, a rodent paradigm for a hippocampus-dependent performance [33, 34], and a glucose tolerance test, followed by blood collection for the analysis of leptin, triglyceride, and cholesterol content (study $2 ; \mathrm{n}=8$ for the control group and $\mathrm{n}=$ 7 for the group fed with a high-cholesterol and fat-containing diet; Figure 1(e)). Finally, a portion of mice was sacrificed and dissected for the analysis of gene expression of a mitochondrial activity marker PPARGC1a, in the prefrontal cortex, hippocampus, hypothalamus, dorsal raphe, and liver (study $3 ; \mathrm{n}=5$ for each group; Figure 1(f)). In the first two experiments, behavioural and biochemical assays were carried out during four consequent days following a period of a dietary intervention; gene expression was analysed at the midpoint of this testing period (Figures 1(d), 1(e), and 1(f)). 

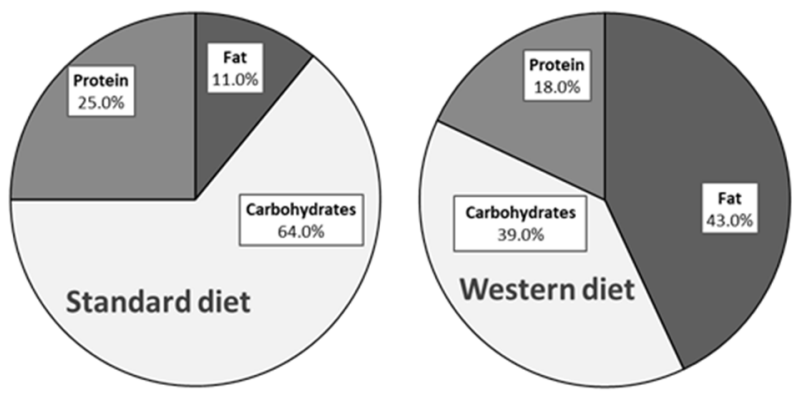

(a)



(b)

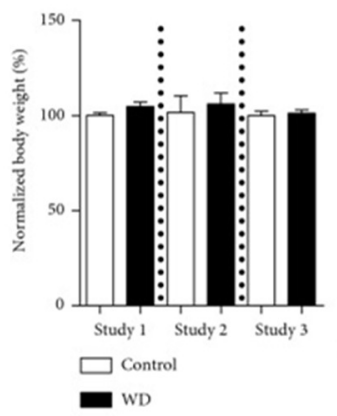

(c)

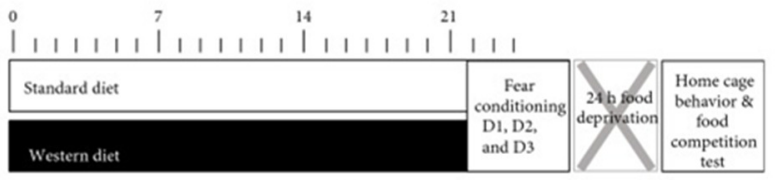

(d)

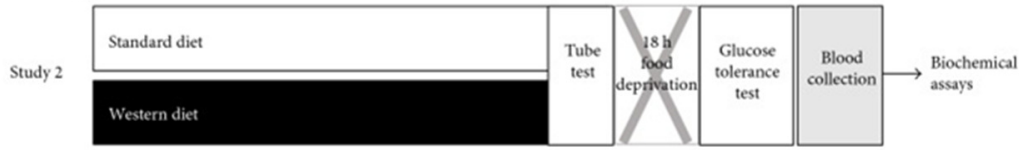

(e)



(f)

Figure 1. Experimental design. (a) Composition of the diets and percentage of total calories. Food intake (b) and body weight (c) normalized to control on day 21 of a dietary challenge (control - control diet, WD - Western diet; *p $<0.05$ versus that in the control diet, t-test). (d) Study of the effects of high-fat/highcholesterol diet exposure on mouse behaviour in fear conditioning test, home cage, and food competition test. (e) Study of the effects of high-fat/high-cholesterol diet exposure on mouse performance in pellet displacement tube test, glucose tolerance, and blood biochemical parameters. (f) Study of the effects of cholesterol-enriched diet exposure on gene expression in the brain and liver. 


\subsection{Behavioural Testing}

All behavioural tests were carried out during an active period of animals' light cycle (09:00-21:00); behaviour was recorded and manually scored offline using behavioural criteria that were previously validated with automated scoring $[35,36]$. The experimenter was blind for the diet used in the mice subjected to the testing.

\subsubsection{Social interactions and solitary behavioural activities in a home cage}

In both experimental groups, social behaviours and other activities in a home cage were assessed $24 \mathrm{~h}$ after a food deprivation, immediately prior to the food competition test. Mice were fooddeprived in order to potentiate their social interactions [32]. The top of a home cage was replaced by a transparent cover, and mouse behaviours were recorded during 10 min under the subtle lighting (light intensity 5 lux). The following behavioural parameters were evaluated: group huddle, "sitting alone", time spent in motion, and digging behaviour. The group huddle was defined as sitting of a mouse in a physical contact to a body of at least one cage mate [36, 37]. "Sitting alone" defined as a position of a mouse in a cage without any physical contacts to another cage mate(s). Time spent in motion was defined as an appearance of horizontal movement of an animal where a position of a centre of a body changed in a cage with a speed $>2 \mathrm{~cm} / \mathrm{sec}$ or an animal would display a rearing by taking a vertical position for $>1 \mathrm{sec}$, 
as defined previously [38]. To evaluate digging (burrowing) behaviour, a species-specific behaviour of a displacement of bedding material using the snout and forepaws, percentage of mice that display this form of behaviour was calculated in each group; its total duration for an observation period was evaluated as well.

\subsubsection{Social interactions during a food competition test}

In the food competition test, two $24 \mathrm{~h}$ food-deprived mice from different cages and the same experimental group were placed in a plastic observation cage $(21 \mathrm{~cm} \times 27 \mathrm{~cm} \times 14 \mathrm{~cm})$ which contained a piece of beef meat $(2 \mathrm{~g})$ for 10 min under subtle lighting (light intensity:

5 lux) and allowed to compete for food as described previously [32]. Our previous studies showed that these conditions, which were adapted from earlier studies [39], female mice which are genetically prone to display aggression, are triggered for agonistic behaviours, competition for food, and aggressive behaviours (Strekalova et al., unpublished data). While previous studies revealed a lack of direct evidences for proaggressive effects of a high-fat/cholesterol diet on female mice in a food competition test [32], the same paradigm was used to study detailed analysis of social interactions in the current study.

During this test, animals were analysed for crawl-over behaviour, as well as body-body, nose-anal, and nose-nose contacts as described elsewhere [40]. The crawl-over behaviour was defined as climbing over the back and head of another animal. "Nose-nose" contacts were defined 
as maintaining vibrissae for longer than 1 sec. "Nose-anal" contacts were defined as an examination of the anogenital area of another mouse. "Body-body" contacts were defined as other physical contacts that did not fall under the criteria of above-described forms of interactions. For each type of social contacts, the latency, duration, and a number of behavioural events were scored.

Additionally, during a food competition test, each mouse was scored for horizontal activity by counting the number of lines crossed where squares were app $10 \times 10 \mathrm{~cm}$ in size, for the entire observation period. Their vertical activity was scored by counting the total number of rearings; a latency to rear was registered as well.

\subsubsection{Acquisition and extinction of contextual fear in the fear conditioning paradigm}

The apparatus (Open Science, Russia and Technosmart, Rome, Italy) consisted of a transparent plastic cubicle $(25 \times 25 \times 50 \mathrm{~cm})$ with a stainless-steel grid floor (33 rods, $2 \mathrm{~mm}$ in diameter). A single alternating electric current (AC, $50 \mathrm{~Hz} ; 0.5 \mathrm{~mA}, 1 \mathrm{sec}$, Evolocus LLC, Tarrytown, NY, USA) was delivered after a 2 min acclimatization period. After delivery of the current, the mouse was immediately placed back in the home cage. Freezing behaviour was scored by visual observation during a test of memory recall that was carried out $24 \mathrm{~h}$ later as described elsewhere [41, 42]. The occurrence of freezing behaviour was assessed every $10 \mathrm{~s}$ for $180 \mathrm{~s}$; each $10 \mathrm{~s}$ score was assigned 
to a freezing or nonfreezing period, and the percentage of time spent in freezing was calculated. Mice that spent $\geq 50 \%$ of time in freezing were defined as "good learners". After scoring of memory recall, mice were left for another seven minutes in the apparatus for memory extinction; no foot shock was applied during this period. $24 \mathrm{~h}$ later, freezing behaviour was scored again in a $180 \mathrm{~s}$ recall of extinction session as in the previous trial and percentage of time spent in freezing was calculated.

\subsubsection{Pellet displacement tube test}

In order to further assess hippocampal function, all experimental groups were tested for pellet displacement in a tube test [33, 34]. A tendency to displace small objects, for example, small stones or food pellets from a tube inside the cage, is species-specific in mice and has been demonstrated to depend on an intact hippocampal formation [33]. Using a paper tube (internal diameter $4 \mathrm{~cm}$, length $10 \mathrm{~cm}$ ), filled with 20 food pellets and placed in the middle of a cage $(21 \mathrm{~cm} \times 27 \mathrm{~cm} \times 14 \mathrm{~cm})$, the latency to displace the first food pellet and time required for $50 \%$ and $100 \%$ tube emptying were assessed in mice.

\subsection{Glucose tolerance test}

The animals from both groups underwent an oral glucose tolerance test. Mice were fasted overnight during $18 \mathrm{~h}$, starting at 17:00; thereafter, glucose solution $(2 \mathrm{~g} / \mathrm{kg}, 1.8 \mathrm{~g} / \mathrm{l})$ was delivered into 
the stomach by oral gavage and blood was sampled from the tail vein. Samples were obtained before glucose administration at time point 0 and 5, 15, 30, 60, 90, and 120 minutes after. The level of blood glucose was analysed using the OneTouch UltraEasy glucometer and strips (LifeScan OneTouch, Dubai, UAE). Because there were no group differences between basal blood glucose levels, absolute values of glucose concentrations were analysed. The area under a curve for the dynamics of this parameter was calculated as well.

\subsection{Brain dissection, RNA extraction, and RT-qPCR}

Mice were sacrificed by cervical dislocation as described elsewhere $[30,40]$. The brain of each mouse was dissected, and the prefrontal cortex was isolated and stored at $-80^{\circ} \mathrm{C}$ until use. mRNA was extracted by using TRI Reagent (MRC, USA). First-strand cDNA synthesis was performed using random primers and Superscript III transcriptase (Invitrogen, Darmstadt, Germany); $1 \mu \mathrm{g}$ total RNA was converted into cDNA. Quantitative PCR for the peroxisome proliferator-activated receptor gamma coactivator 1-alpha (PPARGC1a) gene and the housekeeping gene glyceraldehyde 3-phosphate dehydrogenase (GAPDH) was performed using the SYBR Green master mix (Bio-Rad Laboratories, Philadelphia, USA) and the CFX96 Real-time System (Bio-Rad Laboratories, Philadelphia, USA). Sequences of primers used

are indicated in Supplementary Table 2. Data were normalized to GAPDH mRNA expression and calculated as relative fold changes 
compared to that in control mice as described elsewhere [30, 40]. Results of qRT-PCR measurement were expressed as Ct values, where Ct is defined as the threshold cycle of PCR at which amplified product was $0.05 \%$ of normalized maximal signal. We used the comparative Ct method and computed the difference between the expression of the gene of interest and GAPDH in each cDNA sample (2- $\Delta \Delta \mathrm{Ct}$ method). Data are given as expression folds compared to the mean expression values in control mice.

\subsection{Blood biochemical analysis}

Trunk blood was collected by decapitation during animals' sacrifice, stored at $4^{\circ} \mathrm{C}$ overnight, and centrifuged at $10000 \mathrm{~g}$ for $10 \mathrm{~min}$ at $4^{\circ} \mathrm{C}$. Serum was collected and stored at $-20^{\circ} \mathrm{C}$ until use. A commercially available Mouse Leptin (OB) ELISA Kit (Sigma-Aldrich, MA, USA) was used to measure leptin level; the optical densities of experimental plates were measured at $450 \mathrm{~nm}$ using a plate reader (Wallac 1420 VICTOR, Waltham, MA, USA). All samples were run in duplicate. Quantitative determination of cholesterol and triglycerides in mouse serum was performed on a biochemistry analyzer Konelab 30i (Thermo Fisher Scientific, MA, USA) using a cholesterol kit and triglyceride kit (Thermo Fisher Scientific). All procedures were done according to the instruction manual. 


\subsection{Statistics}

Data were analysed using GraphPad Prism version 5.0 (San Diego, CA, USA). All quantitative data sets were first analysed for normal distribution using Shapiro-Wilk normality test; then, t-test and two-way ANOVA with Bonferroni post hoc testing were used for normally distributed data and Mann-Whitney test for not normally distributed data. Two-way ANOVA was used to analyse the results from the glucose tolerance test. Fisher's exact test was used for categorical data. The level of significance was set at $\mathrm{p}<0.05$.

\section{Results}

\subsection{Changes in food intake and body weight}

In accordance with the previous findings, intake of the high-cholesterol and fat-containing diet was lower than that of the control diet, likely due to the adjustment of mice to the higher caloric value of the WD. This difference was significant in studies 2 and 3 (t $=3.35, \mathrm{p}=0.004$ and $\mathrm{t}=5.97, \mathrm{p}=0.0001$, respectively, Figure $\mathbf{1}(\mathbf{b})$ ) and close to a level of significance in study $2(\mathrm{t}=2.18, \mathrm{p}=0.061)$. Body weight did not differ significantly between groups in all three studies, suggesting that the dietary intervention did not grossly alter body composition ( $\mathrm{p}>0.05$, t-test, Figure 1(c)); however, a tendency to an increased body mass was found in study $1(\mathrm{t}=1.79, \mathrm{p}=0.093)$. These findings are in line with our previous results that showed a lack 
of body weight changes and a decrease in intake of a high-cholesterol and fat-containing diet [24-26].

3.2. Aberrant social interactions, hyperlocomotion, and excessive digging behaviour in home cage conditions and during the food competition test in dietary-induced mice

During study 1, we found that in a home cage, in comparison with controls, mice fed with the high-cholesterol and fat-containing diet showed a significant decrease in the duration of group huddle behaviour $(\mathrm{t}=16.49, \mathrm{p}<0.001$, t-test; Figure $\mathbf{2}(\mathbf{a}))$ and a significant increase in the duration of "sitting alone" behaviour $(\mathrm{t}=5.81$, $\mathrm{p}<0.001$, t-test; Figure 2(b)), as well as the time spent in motion $(\mathrm{t}=13.39, \mathrm{p}<0.00, \mathrm{t}$-test; Figure $\mathbf{2}(\mathbf{c}))$. The number of animals expressing digging behaviour during this test and duration of digging (burrowing) behaviour, which is a sign of active copying and a correlate of social dominance, were significantly increased in the group fed with the fat/cholesterol-enriched diet compared to the control $(\mathrm{p}=0.003$, Fisher's exact test and $\mathrm{U}=24.00, \mathrm{p}=0.003$, Mann-Whitney test, Figures 2(d) and 2(e)). Thus, consumption of the high-cholesterol and fat-containing diet induces social avoidance, hyperlocomotion, and excessive digging behaviour in home cage conditions. 


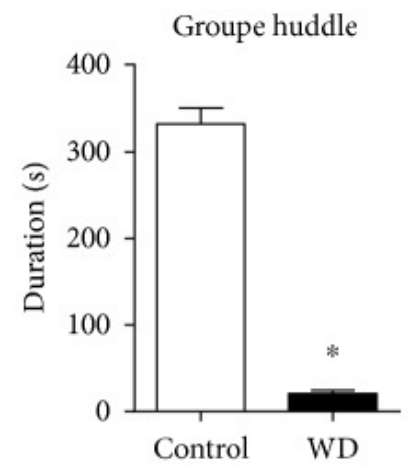

(a)

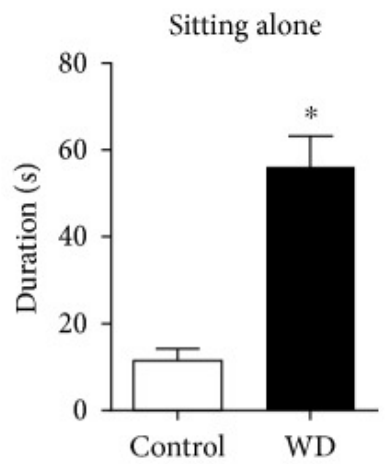

(b)
Time spent in motion

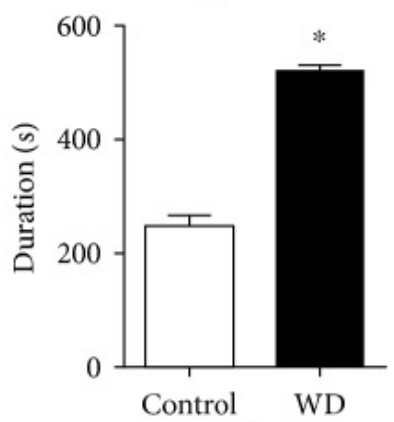

(c)

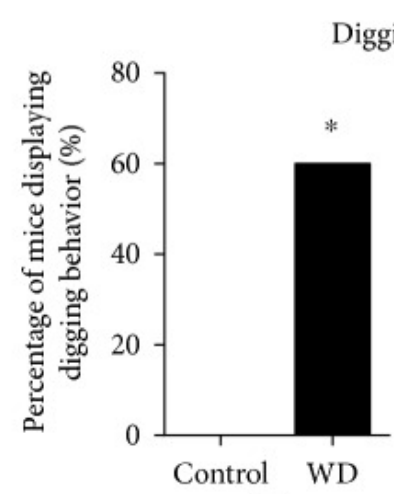

(d)

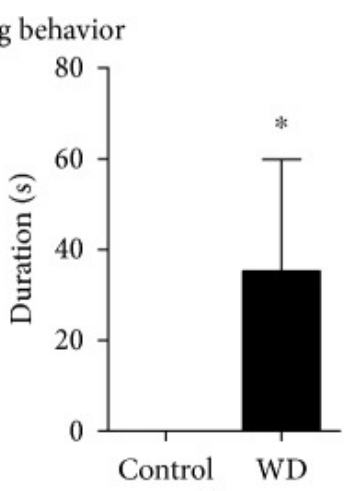

(e)

Figure 2. Dietary challenge with the Western diet results in aberrant home cage social behaviour. In comparison to control mice, the dietarychallenged group displayed (a) a significant decrease in the duration of group huddle, (b) a significant prolongation of the duration of "sitting alone" behaviour, and $(\mathrm{c})$ a significant increase in the time spent in motion in the cage $(* \mathrm{p}<0.05$ versus that in the control group, t-test). As compared to control animals, a group fed with the high-fat/high-cholesterol diet showed a significant elevation of (d) the percentage of mice displaying digging behaviour $(* \mathrm{p}<0.05$ versus that in the control group, Fisher's exact test) and (e) the duration of digging behaviour $(* \mathrm{p}<0.05$ versus that in the control group, Mann-Whitney test). Controlstandard diet, WD-Western diet. Data are shown as mean $\pm \mathrm{SEM}$. 
During the food competition test, in comparison with controls, the dietary-challenged group showed a significant decrease in the latency of crawl-over behaviour $(\mathrm{U}=25.50, \mathrm{p}=0.020$; Mann-Whitney test, Figure 3(a)) and a significant increase in the number of crawlover behavioural events $(\mathrm{U}=25.50, \mathrm{p}=0.020$, Mann-Whitney test, Figure 3(b)) and the duration of this behaviour $(\mathrm{U}=19.00, \mathrm{p}=$ 0.005, Mann-Whitney test, Figure 3(c)). In comparison to mice housed on a regular diet, mice fed with the high-cholesterol and fatcontaining diet showed no difference in the latency of body-body contacts between the groups $(\mathrm{U}=10.00, \mathrm{p}=0.398$; Mann-Whitney test, Figure 3(d)); however, the number of the body-body contacts was significantly increased $(\mathrm{U}=3.00, \mathrm{p}=0.026$; Mann-Whitney test, Figure 3(e)), while the total duration of this behaviour was decreased $(\mathrm{U}=1.00, \mathrm{p}=0.009$, Mann-Whitney test, Figure 3(f)). The latency, number, and duration of nose-anal and nose-nose contacts were not different between the groups $(\mathrm{U}=49.50, \mathrm{p}=0.502 ; \mathrm{U}=53.50, \mathrm{p}=$ 0.615; $\mathrm{U}=53.50, \mathrm{p}=0.615$ and $\mathrm{U}=10.00, \mathrm{p}=0.416 ; \mathrm{U}=7.00, \mathrm{p}=$ 0.171; $\mathrm{U}=8.00, \mathrm{p}=0.197$, respectively; Mann-Whitney test, Figures $\mathbf{3}(\mathrm{g}), \mathbf{3}(\mathrm{h}), \mathbf{3}(\mathrm{i}), \mathbf{3}(\mathrm{j}), \mathbf{3}(\mathrm{k})$, and $\mathbf{3}(\mathrm{l}))$. 


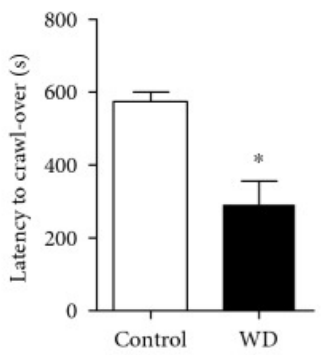

(a)

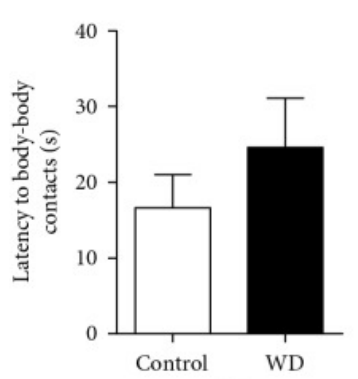

(d)

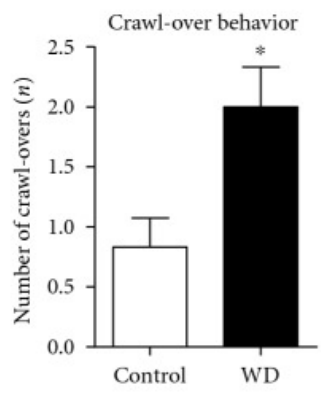

(b)

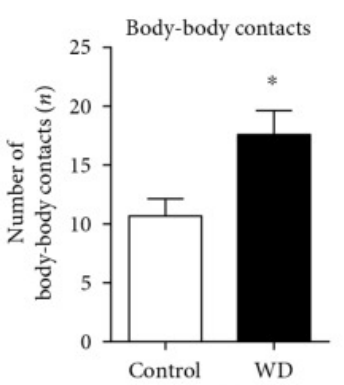

(e)

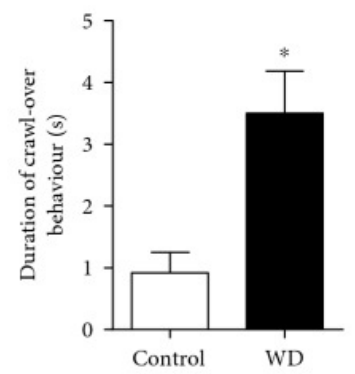

(c)

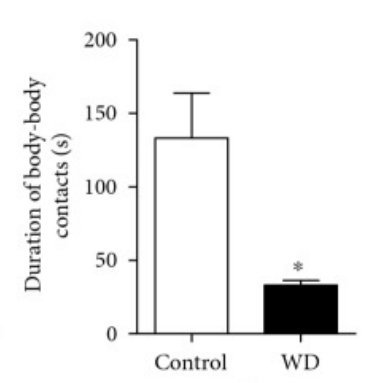

(f)

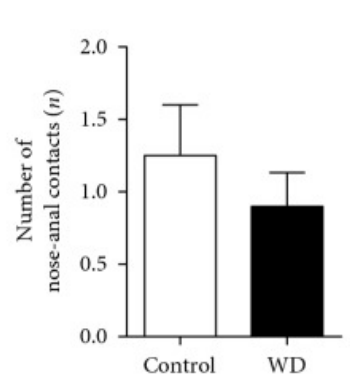

(g)

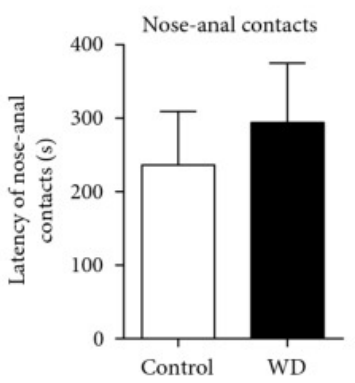

(h)

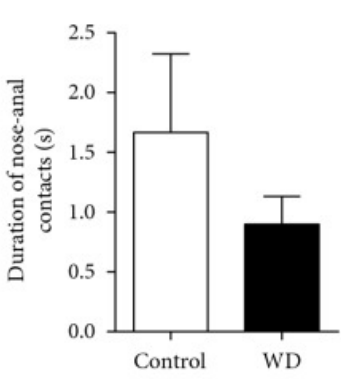

(i)

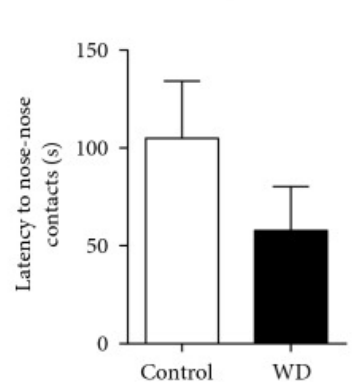

(j)

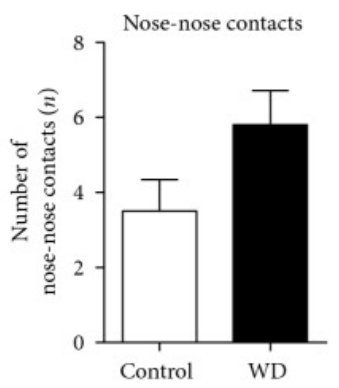

(k)

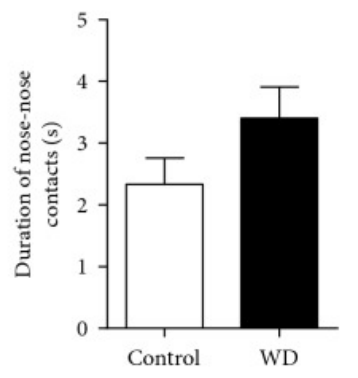

(I)

Figure 3. The high-fat/high-cholesterol diet potentiates dominant-like behaviour and suppresses sociability in a food competition test. In comparison to the control group, mice housed on the high-fat/high-cholesterol diet 
showed (a) a significant decrease in the latency to crawl-over behaviour and (b) a significant increase in a number of crawl-overs, as well as (c) prolonged total duration of crawl-over behaviour $(* \mathrm{p}<0.05$ versus that in the control group; Mann-Whitney test). In comparison to the control group, mice exposed to the high-fat/high-cholesterol diet had (d) no significant changes in the latency to bodybody contacts and had (e) a significant increase in a number of body-body contacts $(* \mathrm{p}<0.05$ versus that in the control group; Mann-Whitney test) and (f) a significant decrease in the duration of body-body contacts $(* \mathrm{p}<0.05$ versus that in the control group; Mann-Whitney test). No significant group differences were observed between the groups in the parameters of nose-anal contacts: $(\mathrm{g})$ the number of nose-anal contacts, (h) latency to nose-anal contacts, and (i) total duration of nose-anal contacts $(* \mathrm{p}>0.05$ versus that in the control group; Mann-Whitney test). There were no significant group differences in the parameters of nose-nose contacts: (j) latency of nose-nose contacts, $(\mathrm{k})$ number of nose-nose contacts, and (l) total duration of nose-nose contacts $(* \mathrm{p}>0.05$ versus that in the control group; Mann-Whitney test). Control—standard diet, WD-Western diet. Data are shown as mean \pm SEMs.

During the food competition test, in comparison with control mice, the group fed with the high-cholesterol and fat-containing diet showed a significant increase in the total number of line crossings $(\mathrm{t}=4.18, \mathrm{p}<0.001, \mathrm{t}$-test, Figure $4(\mathbf{a}))$ and in the total number of rearings $(\mathrm{U}=17.50, \mathrm{p}=0.004$, Mann-Whitney test, Figure $4(\mathrm{~b}))$ and a significant decrease in the latency of the first rearing event $(\mathrm{t}=4.42$, $\mathrm{p}=0.001$, t-test, Figure $4(\mathbf{c}))$. Together, these data suggest increased dominant-like behaviours, reduced sociability associated with abnormal social behaviour, and hyperlocomotion in mice housed on the WD. 
Horizontal activity



(a)

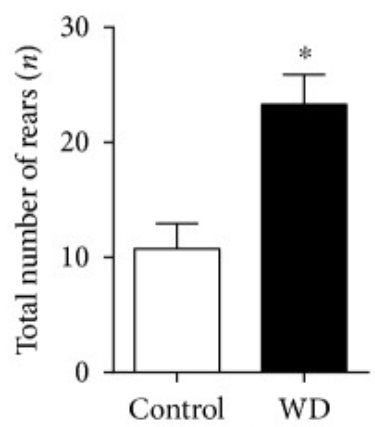

(b)
Vertical activity

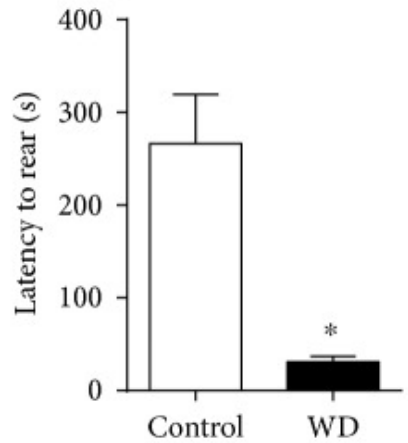

(c)

Figure 4. The high-fat/high-cholesterol diet enhances horizontal and vertical activity during food competition test. In comparison to control mice, animals exposed to the high-fat/high-cholesterol diet showed (a) a significant increase in the total number of line crossing $(* \mathrm{p}<0.05$ versus that in the control group, t-test), (b) significant elevation of the total number of rearings $(* \mathrm{p}<0.05$ versus that in the control group, Mann-Whitney test), (c) and a significant decrease in the latency of rearings $(* \mathrm{p}<0.05$ versus that in the control group, t-test). Control-standard diet, WD-Western diet. Data are shown as mean \pm SEM (a, c) and median with interquartile range (b).

3.3. Exposure to a diet enriched with fat and cholesterol results in the deficient hippocampus-dependent performance in the fear conditioning and pellet displacement tests

In the fear conditioning test, in comparison to that in control mice, the number of "good learners" defined by the percentage of time spent with freezing $\geq 50 \%$ at the memory recall session was significantly diminished in the dietary-challenged group $(\mathrm{p}=0.017$, Fisher's exact test, Figure 5(a)); also, there was a strong trend to a decreased duration of freezing in this group $(\mathrm{t}=1.96, \mathrm{p}=0.059$, t-test, 
Figure 5(b)), suggesting reduced contextual memory in mice housed on the high-cholesterol and fat-containing diet.

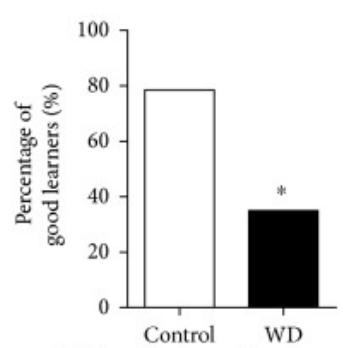

(a) Recall of conditioning $+24 \mathrm{~h}$ posttraining

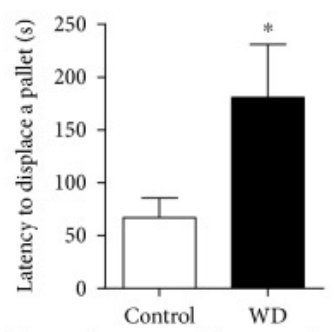

(d) Displacement of the 1st pellet

Contextual fear conditioning and extinction



(b) Recall of conditioning $+24 \mathrm{~h}$ posttraining

Pellet displacement test

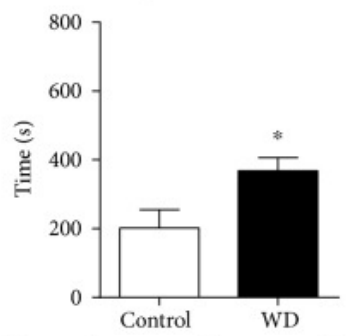

(e) Displacement of the $50 \%$ pellet

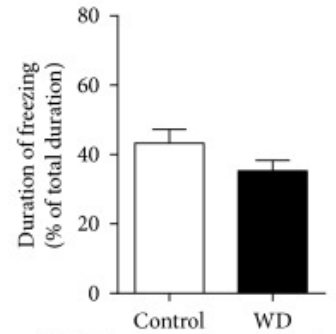

(c) Extinction of conditioning $+48 \mathrm{~h}$ posttraining



(f) Displacement of the $100 \%$ pellet

Figure 5. The high-fat/high-cholesterol diet compromises the hippocampus-dependent performance. In the fear conditioning test, in comparison to control mice, the dietary-challenged group showed (a) significant decreased number of "good learners" $(* \mathrm{p}<0.05$ versus that in the control group, Fisher's exact test), (b) a strong tendency to a reduced time spent with freezing during recall of conditioning ( $\mathrm{p}=0.055$ versus that in the control group, $\mathrm{t}$-test), and (c) a significant decrease in time spent with freezing in a memory extinction protocol $(* \mathrm{p}<0.05$ versus that in the control group, t-test). In the pellet displacement tube test, in comparison to control animals, mice exposed to the high-fat/high-cholesterol diet showed significantly prolonged (d) latency of a displacement of the 1st pellet and (e) the duration of displacement of $50 \%$ pellets $(* \mathrm{p}<0.05$ versus that in the control group, t-test) and (f) did not differ in the duration of a displacement of $100 \%$ pellets ( $p>0.05$ versus that in the control group, t-test). Control - standard diet, WD-Western diet. Data are shown as mean \pm SEM. 
During the recall of memory extinction, mice housed on the WD showed significantly shorter duration of freezing than control mice, suggesting faster extinguishing of contextual memory due to its weaker acquisition $(\mathrm{t}=3.024, \mathrm{p}=0.005$, t-test, Figure 5(c)). During study 2 , in the pellet displacement tube test, the latency to displace a pellet and time required for a $50 \%$ emptying the tube with pellets were significantly increased in the high-fat/high-cholesterol diet group, in comparison to control animals $(\mathrm{t}=2.26, \mathrm{p}=0.044$ and $\mathrm{t}=2.62$, $\mathrm{p}=0.020$, respectively, t-test, Figures $\mathbf{5}(\mathbf{d})$ and $\mathbf{5}(\mathbf{e}))$. The time required for a $100 \%$ emptying of the tube did not differ significantly between the groups $(\mathrm{t}=1.74, \mathrm{p}=0.105$, t-test, Figure $\mathbf{5}(\mathbf{f})$ ). Together, these data suggest a moderate deficiency in the hippocampus-dependent performance in mice fed with the highcholesterol and fat-containing diet.

\subsection{Altered central and peripheral metabolic markers in mice housed on the high-cholesterol and fat-containing diet}

At study 2, in the glucose tolerance test, two-way ANOVA revealed a significant effect of both the diet and time after glucose load on blood glucose level $(\mathrm{F}=10.16, \mathrm{p}=0.013$ and $\mathrm{F}=19.15, \mathrm{p}<0.001$; Figure 6(a)). 


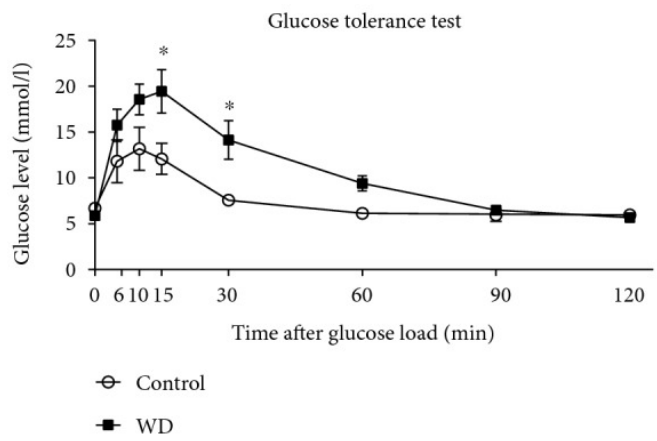

(a)

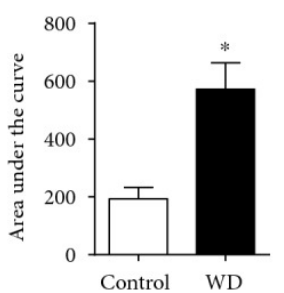

(b)



(c)

Biochemical blood parameters

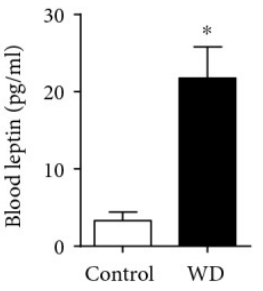

(d)

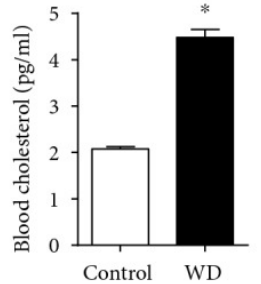

(e)



(f)

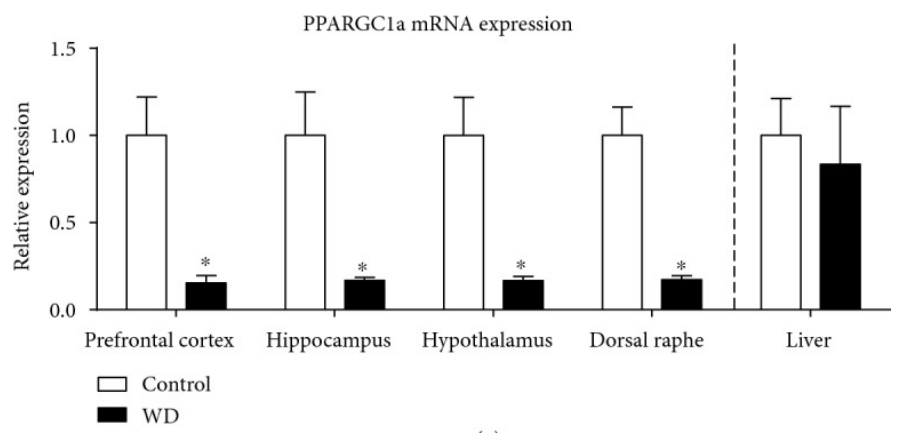

(g)

Figure 6. Effects of high-fat/high-cholesterol diet exposure on glucose tolerance, biochemical blood parameters, and PPARGC1a gene expression. (a) In comparison to that in the control, there was an increase in 
blood glucose level at 15 and 30 min after glucose load in the glucose tolerance test $(* \mathrm{p}<0.05$ versus that in the control group, 2-way ANOVA and Bonferroni post hoc test). (b) There was a significant increase in the area under curve in glucose concentration in dietary-challenged mice in comparison to controls $(* \mathrm{p}<0.05$ versus control group, t-test). (c) There was no significant difference in basal glucose levels between mice housed on the standard and high-fat/high-cholesterol diets. Mice housed on the high-fat/high-cholesterol diet, as compared to the control group, showed significantly increased (d) blood leptin levels, (e) blood cholesterol levels $(* \mathrm{p}<0.05$ versus that in the control group, t-test), and (f) unaltered blood level of triglycerides ( $p>0.05$ versus that in the control group, t-test). (f) In comparison to control mice, animals fed with the high-fat/high-cholesterol diet had significantly decreased PPARGC1a mRNA in all brain areas $(* \mathrm{p}<0.05$ versus that in the control group, 2-way ANOVA and Bonferroni post hoc test) but not in the liver ( $\mathrm{p}>0.05$ versus that in the control group, 2-way ANOVA and Bonferroni post hoc test). Control-standard diet, WD-Western diet. Data are shown as mean \pm SEM.

In comparison with control mice, the dietary-challenged group showed a significant increase in blood glucose levels at the time points 15 and $30 \mathrm{~min}$ of the test and a tendency to increase at time point $10 \min (10 \min : \mathrm{t}=2.54, \mathrm{p}=0.108 ; 15 \min : \mathrm{t}=3.48, \mathrm{p}=0.007 ; 30 \min :$ $\mathrm{t}=3.09, \mathrm{p}=0.024$, Bonferroni test), suggesting a decrease in glucose tolerance in the latter group. No significant group differences were found at other time points. The area under a curve calculated for a $2 \mathrm{~h}$ period of the afterload measurements of blood glucose levels normalized to the baseline was significantly increased in the high-cholesterol and high-fat diet group as compared to the control $(\mathrm{t}=3.21, \mathrm{p}=0.012$, t-test, Figure 6(b)). There was no difference in basal glucose level between the groups $(t=1.67, p=0.137$, t-test; Figure 6(c) $)$. Serum levels of leptin and cholesterol were significantly increased in the group 
fed with the high-fat/cholesterol diet as compared to control animals $(\mathrm{t}=4.40, \mathrm{p}=0.037$ and $\mathrm{t}=13.47, \mathrm{p}<0.001$, respectively; t-test;

Figures 6(d) and 6(e)); no difference was found in triglyceride level $(\mathrm{t}=0.68, \mathrm{p}=0.529 ;$ t-test; Figure 6(f) $)$.

Two-way ANOVA showed significant differences between the groups investigated in study 3 in the PPARGC1a mRNA concentrations in the brain $(\mathrm{F}=56.12, \mathrm{p}<0.001)$, which were independent of the brain area $(\mathrm{F}=0.28, \mathrm{p}=0.841$; two-way ANOVA; Figure 6(g)). The PPARGC1a mRNA levels were significantly lower in all investigated brain regions in mice fed with the high-cholesterol and fat-containing diet, in comparison to control mice (prefrontal cortex: $\mathrm{t}=4.00, \mathrm{p}=0.001$; hippocampus: $\mathrm{t}=3.92, \mathrm{p}=0.002$; hypothalamus: $\mathrm{t}=3.93 ; \mathrm{p}=0.002 ;$ and dorsal raphe: $\mathrm{t}=3.13$, $\mathrm{p}=0.015$, Bonferroni test). Hepatic PPARGC1a mRNA levels did not significantly differ between the groups $(\mathrm{t}=0.42, \mathrm{p}=0.687$, t-test $)$.

\section{Discussion}

In this study, we showed, for the first time, that chronic exposure of naïve adult mice in the NAFLD model employed here to a highcholesterol and fat-containing diet induces substantial changes in their social behaviour, comprising of a reduction in sociability and an increase in a dominant-like behaviour. These changes were accompanied by altered patterns of social behaviours and hyperactivity 
in a context of social interactions. Furthermore, dietary challenge with the high-cholesterol and fat-containing diet resulted in a decreased acquisition and enhanced extinction of contextual fear conditioning and slower hippocampus-dependent performance in pellet displacement tube test. Glucose intolerance, elevated plasma leptin and cholesterol levels, and decreased expression of the PPARGC1a gene in the brain but not in the liver paralleled above-described behavioural abnormalities.

The analysis of social interactions both in a home cage and during a food competition test demonstrated that in comparison to control animals, dietary-challenged mice were less sociable, spending less time huddling and longer time "sitting alone" in a home cage, as well as shorter duration of body-body contacts during the food competition model. These data can be interpreted as a sign of social avoidance of both familiar and unfamiliar mice displayed by animals fed with the WD. They also rule out a factor of neophobia that could explain lower social interactions in cases where only the interactions with unfamiliar mouse would be reduced in mice exposed to the WD.

Our results are generally in line with several studies that reported diminished sociability in rodents after exposures to diets containing high amounts of fat/cholesterol; however, previous works largely dealt with their effects on the offspring $[3,15]$. In one of the most recent studies, Buffington and colleagues found that the offspring of dams fed 
with a high-fat diet containing high amounts of cholesterol displayed markedly dropped number, frequency, and duration of social contacts both with familiar and unfamiliar mouse, as well as impaired long-term potentiation in the ventral tegmental area, a sign of a deficient synaptic plasticity [15]. These deficits were rescued by supplementary oxytocin and a restoration of normal microbiota parameters, which were affected in the offspring of dietary-challenged mice and can potentially mediate reported effects here. Moreover, another study with a high-fat diet containing high amounts of cholesterol showed that it can exacerbate social deficiency and cognitive rigidity in BTBR $\mathrm{T}+\mathrm{tf} / \mathrm{J}$ inbreed mouse line, a model of autism. BTBR mice, after housing on a high-fat diet containing high amounts of cholesterol starting at weaning, demonstrated greater deficits in social memory, lowered preference for social novelty, and impaired learning of the T-maze than these mice fed with a regular diet [3].

In the current study, the group fed with the WD revealed heightened scores of crawl-over behaviour in a food competition test and burrowing activity in a home cage, which are generally accounted for the manifestations of a dominant-like behaviour [40, 43]. Burrowing behaviour was shown to correlate with elevated levels of social dominancy and not be displayed by mice with subordinate social traits [43]. Of note, signs of burrowing behaviour were completely absent in the control group in the current study and were expressed only by 
dietary-challenged animals. Other studies suggest that burrowing behaviour is associated with repetitive behaviour and impulsivity that was recently reported in a study with C57 female mice fed with a fat and high-cholesterol diet [44].

In many studies with dietary interventions and assessment of social behaviours, a factor of a gain/loss of body weight was suggested to determine the outcome [45]. In the current work, we choose to apply a dietary challenge that is selectively enhanced in a content of cholesterol and also does not induce a change in body weight, as such, excluding a factor of obesity in the induction of negative consequences of dietary cholesterol. Thus, potential confounds related to altered body mass in the evaluation of social and other behaviours could be ruled out.

Our study has also revealed the alternations in the basic patterns of social interactions. An increase in the number of body-body contacts associated with a decrease in their duration can be interpreted as a sign of impulsive behaviour that was previously shown in an employed model under different experimental settings from those used in the present work, which did not include the elements of social interaction unlike the current work [30]. In line with this result and the previous findings, we found increased vertical and horizontal locomotion during both home cage testing and food competition test in Western dietchallenged mice, suggesting their impulsivity which was displayed in a 
context of social interactions. Thus, mice challenged with high amounts of dietary cholesterol and fat display hyperlocomotion/impulsivity regardless of whether or not social element presents in their environment.

Similar to our results, a combination of exposure to a highfat/high-cholesterol diet with food deprivation was recently found to enhance behavioural signs of impulsivity and upregulation of several molecular factors involved in the pathophysiology of impulsivity and addiction [45, 46]. Exposure of rodents to this type of diet has been shown to evoke substantial alternations in dopamine signalling in several brain structures including the prefrontal cortex [46, 47], the nucleus accumbens [47], and the hypothalamus [48]. As these changes have been associated with the mechanisms in social interactions, motivation, and various other behaviours [49-52], they can underlie the above-described behavioural signs of hyperactivity and impulsivity observed in our and previous studies.

Increased rates of dominant behaviours and decreased signs of sociability can be related to deficient cognitive functions in general and hippocampal plasticity in particular $[17,18]$. The current study showed that applied exposure to a high-fat/high-cholesterol diet reduces contextual fear conditioning in mice that is in line with the previous findings in this paradigm $[21,53]$. Mice housed on a diet enriched with cholesterol were previously found to show increased anxiety [30, 31] 
generally known to potentiate fear learning. Yet, animals from this group displayed lowered percentage of "good learners" and a strong tendency to a reduction in freezing behaviour, suggesting deficient contextual learning. Significantly increased extinction of contextual conditioning as shown by diminished duration of freezing behaviour further suggests weaker learning abilities of mice housed on the highfat/high-cholesterol diet. Other animal studies found altered learning deficits in mice and rats that were housed on a high-cholesterol/highfat diet and tested in a range of learning and memory tasks including water mazes, variable interval delayed alternation task, object recognition test, and operant bar-pressing task [7-12, 54, 55].

Findings described here in the fear conditioning test were further supported by the data obtained in the pellet displacement tube test. Dietary-challenged mice showed a slower performance of emptying a tube filled with pellets in this test, which is regarded as a sign of reduced hippocampus-dependent functions. Previous studies validated the food pellet displacement test as a paradigm, in which rodent behaviour of object displacement from a tube was found sensitive to the lesions of dorsal hippocampus [33] and accompanies the deficits in the hippocampal plasticity during depressive-like syndrome [34] and in a model of Alzheimer's disease [56]. Pellet displacement in rodents was shown to be suppressed by systemic inflammation [56, 57], a feature of animal models of a high-fat/high-cholesterol diet [6, 9, 26, 30]. 
As deficient cognition, reduced exploration, aggressive behaviour, attention deficit, depressive-like and anxiety-like changes, neophobia, and impulsivity constitute the elements of autistic behavioural profile $[18,58]$, behavioural changes in mice fed with the WD resembled proautistic behavioural repertoire.

Behavioural changes found in mice exposed to dietary cholesterol and fat were accompanied by pronounced metabolic changes. We found increased blood concentrations of cholesterol and leptin, while the triglyceride level was unaltered. Dietary-induced hyperleptinemia and hypercholesterolemia were reported to be consistent in high-fat/highcholesterol models $[22,59,60]$, whereas similar to our study, the changes in blood triglyceride levels can vary $[22,59]$.

The present work has demonstrated delayed restoration of normal glucose levels after glucose load in the WD-challenged mice, suggesting their impaired glucose tolerance and insulin resistance that were not reported before in the NAFLD model employed here. Together with marketable inflammation that was previously demonstrated in an applied model here [30] and that can be a causal factor of insulin resistance [61], this finding led to suggesting altered insulin-mediated signalling in the brain, where insulin resistance in the hippocampus may account for reported behavioural abnormalities here. The use of high-fat/high-cholesterol diets showed that behavioural changes of rodents housed on these diets are accompanied by type 2 diabetes 
mellitus [8, 10, 21, 53]. Importantly, heightened occurrence of diabetes and reduced glucose tolerance during autistic spectrum disorder is well established in the literature $[62,63]$.

Epidemiological and clinical studies provide evidence for the role of metabolic syndrome on the occurrence of dementia and cognitive decline, in particular in the presence of inflammation [64, 65]. Mitochondrial dysfunction was found to be associated with social deficits in young individuals $[62,66,67]$ and cognitive impairment in aging people and animals $[68,69]$. Clinical and animal studies suggest a common neurobiological basis and interrelationship for aggression, diminished cognitive abilities, behavioural disinhibition, and metabolic abnormalities. Remarkably, pharmacological enhancement of insulin receptor signalling was demonstrated to rescue normal emotional behaviours in an employed paradigm here [31]. Since central and peripheral glucose intolerance is well documented to underlie numerous affective and cognitive abnormalities [70-74], this mechanism is likely to explain described behavioural aberrations here.

Our study revealed a decrease in PPARGC1a, a marker of diminished mitochondrial activity during dietary-induced type 2 diabetes $[16,31,75,76]$, in the brain of mice housed on the highfat/high-cholesterol diet. This finding is in line with reported reduced glucose tolerance here in the WD group and further supports the view that suppressed mitochondrial functions in the brain may underlie 
described behavioural abnormalities here of mice housed on the WD. Interestingly, diminished expression of this gene was also found in the human hippocampus, correlating with clinical progression of dementia in patients with Alzheimer's disorder [27]. Mice genetically lacking the PPARGC1a gene exhibited an imbalance between inhibitory and excitatory synaptic transmission in the hippocampus, a mechanism that is suggested to be an important pathogenetic factor of autism [28, 77]. Thus, cognitive and other behavioural deficits reported in this work on mice fed with the high-fat/high-cholesterol diet can result not only from lowered mitochondrial activity that is associated with decreased PPARGC1a brain levels but also specific roles of this molecule in synaptic plasticity.

Previously, we showed a decreased gene expression in an employed model here of another member of peroxisome proliferatoractivated receptor family member, PPARGC1b, both in the brain and in the liver [31], whereas no changes in the hepatic PPARGC1a were found in the present study. These results highlight functional differences between two molecules as suggested earlier [31, 77].

Together, our data are generally consistent with so far obtained results with rodent models suggesting that diets containing high amounts of fat/cholesterol can evoke social deficiencies which resemble the features of autism spectrum disorders. Our results correspond to the current epidemiological findings, demonstrating a link between 
obesity and autism [14, 62], and to reports on ameliorated cognitive and behavioural symptoms of autism due to a diet [63]. Thus, the results reported here, along with currently accumulated data considering behavioural abnormalities in the light of proautistic changes, allow speculation that preference for the "Western diet" can be a potential risk factor for this spectrum of diseases in humans. These findings provide evidence for a possible environmental risk factor that can contribute to the production of autistic-like symptoms.

\section{Acknowledgments}

The authors' work reported in this paper was supported by the European Community (EC: AGGRESSOTYPE FP7/no. 602805 and Eat2beNICE Horizon 2020/no. 677302) and Russian Research Excellence project "5-100". This publication was funded by the German Research Foundation (DFG) and the University of Wuerzburg in the funding programme Open Access Publishing.

\section{Conflicts of Interest}

All contributing authors have no conflict of interests.

\section{References}

1. Sonawalla S. B., Papakostas G. I., Petersen T. J., et al. Elevated cholesterol levels associated with nonresponse to fluoxetine treatment in major depressive disorder. Psychosomatics. 2002;43(4):310-316. doi: 10.1176/appi.psy.43.4.310. 
2. Felger J. C., Lotrich F. E. Inflammatory cytokines in depression: neurobiological mechanisms and therapeutic implications. Neuroscience. 2013;246:199-229. doi: 10.1016/j.neuroscience.2013.04.060.

3. Zilkha N., Kuperman Y., Kimchi T. High-fat diet exacerbates cognitive rigidity and social deficiency in the BTBR mouse model of autism. Neuroscience. 2016;345:142-154. doi: 10.1016/j.conb.2016.04.014.

4. Pohl J., Sheppard M., Luheshi G. N., Woodside B. Diet-induced weight gain produces a graded increase in behavioral responses to an acute immune challenge. Brain, Behavior, and Immunity. 2014;35:43-50.

doi: 10.1016/j.bbi.2013.09.002.

5. Parrott M. D., Greenwood C. E. Dietary influences on cognitive function with aging: from high-fat diets to healthful eating. Annals of the New York Academy of Sciences. 2007;1114(1):389-397. doi: 10.1196/annals.1396.028.

6. Comhair T. M., Garcia Caraballo S. C., Dejong C. H., Lamers W. H., Köhler S. E. Dietary cholesterol, female gender and n-3 fatty acid deficiency are more important factors in the development of non-alcoholic fatty liver disease than the saturation index of the fat. Nutrition \& Metabolism (London) 2011;8(1):p. 4. doi: 10.1016/j.clnu.2015.11.005.

7. Winocur G., Greenwood C. E. The effects of high fat diets and environmental influences on cognitive performance in rats. Behavioural Brain Research. 1999;101(2):153-161. doi: 10.1016/S0166-4328(98)00147-8.

8. Mielke J. G., Nicolitch K., Avellaneda V., et al. Longitudinal study of the effects of a high-fat diet on glucose regulation, hippocampal function, and cerebral insulin sensitivity in C57BL/6 mice. Behavioural Brain Research. 2006;175(2):374-382. doi: 10.1016/j.bbr.2006.09.010.

9. Thirumangalakudi L., Prakasam A., Zhang R., et al. High cholesterolinduced neuroinflammation and amyloid precursor protein processing correlate with loss of working memory in mice. Journal of Neurochemistry. 2008;106(1):475-485. doi: 10.1111/j.1471-4159.2008.05415.x.

10. Winocur G., Greenwood C. E. Studies of the effects of high fat diets on cognitive function in a rat model. Neurobiology of Aging. 2005;26(Supplement 1):46-49. doi: 10.1016/j.neurobiolaging.2005.09.003.

11. Wang Z., Fan J., Wang J., et al. Protective effect of lycopene on high-fat diet-induced cognitive impairment in rats. Neuroscience Letters. 2016;627:185-191. doi: 10.1016/j.neulet.2016.05.014.

12. Ledreux A., Wang X., Schultzberg M., Granholm A. C., Freeman L. R. Detrimental effects of a high fat/high cholesterol diet on memory and 
hippocampal markers in aged rats. Behavioural Brain Research. 2016;312:294-304. doi: 10.1016/j.bbr.2016.06.012.

13. Kaplan J. R., Manuck S. B., Shively C. The effects of fat and cholesterol on social behavior in monkeys. Psychosomatic Medicine. 1991;53(6):634-642. doi: 10.1097/00006842-199111000-00005.

14. Connolly N., Anixt J., Manning P., Ping-I L. D., Marsolo K. A., Bowers K. Maternal metabolic risk factors for autism spectrum disorder - an analysis of electronic medical records and linked birth data. Autism Research. 2016;9(8):829-837. doi: 10.1002/aur.1586.

15. Buffington S. A., Di Prisco G. V., Auchtung T. A., Ajami N. J., Petrosino J. F., Costa-Mattioli M. Microbial reconstitution reverses maternal dietinduced social and synaptic deficits in offspring. Cell. 2016;165(7):1762-1775. doi: 10.1016/j.cell.2016.06.001.

16. Burgueño A. L., Cabrerizo R., Gonzales M. N., Sookoian S., Pirola C. J. Maternal high-fat intake during pregnancy programs metabolic-syndromerelated phenotypes through liver mitochondrial DNA copy number and transcriptional activity of liver PPARGC1A. The Journal of Nutritional Biochemistry. 2013;24(1):6-13. doi: 10.1016/j.jnutbio.2011.12.008.

17. Williams D. L., Goldstein G., Minshew N. J. The profile of memory function in children with autism. Neuropsychology. 2006;20(1):21-29. doi: 10.1037/0894-4105.20.1.21.

18. Solomon M., McCauley J. B., Iosif A. M., Carter C. S., Ragland J. D. Cognitive control and episodic memory in adolescents with autism spectrum disorders. Neuropsychologia. 2016;89:31-41.

doi: 10.1016/j.neuropsychologia.2016.05.013.

19. Dufour F., Liu Q. Y., Gusev P., Alkon D., Atzori M. Cholesterol-enriched diet affects spatial learning and synaptic function in hippocampal synapses. Brain Research. 2006;1103(1):88-98. doi: 10.1016/j.brainres.2006.05.086.

20. Micale V., Scapagnini G., Colombrita C., Mazzola C., Alkon D. L., Drago F. Behavioral effects of dietary cholesterol in rats tested in experimental models of mild stress and cognition tasks. European Neuropsychopharmacology. 2008;18(6):462-471.

doi: 10.1016/j.euroneuro.2007.11.006.

21. Granholm A. C., Bimonte-Nelson H. A., Moore A. B., Nelson M. E., Freeman L. R., Sambamurti K. Effects of a saturated fat and high cholesterol diet on memory and hippocampal morphology in the middle-aged rat. Journal of Alzheimer's Disease. 2008;14(2):133-145. 
22. Hwang L. L., Wang C. H., Li T. L., et al. Sex differences in high-fat dietinduced obesity, metabolic alterations and learning, and synaptic plasticity deficits in mice. Obesity (Silver Spring) 2010;18(3):463-469. doi: 10.1038/oby.2009.273.

23. Gould N. F., Holmes M. K., Fantie B. D., et al. Performance on a virtual reality spatial memory navigation task in depressed patients. The American Journal of Psychiatry. 2007;164(3):516-519. doi: 10.1176/ajp.2007.164.3.516. 24. Scarpulla R. C. Nuclear activators and coactivators in mammalian mitochondrial biogenesis. Biochimica et Biophysica Acta. 2002;1576(1-2):114.

25. Lin J., Wu P. H., Tarr P. T., et al. Defects in adaptive energy metabolism with CNS-linked hyperactivity in PGC-1 $\alpha$ null mice. Cell. 2004;119(1):121135. doi: 10.1016/j.cell.2004.09.013.

26. Leone T. C., Lehman J. J., Finck B. N., et al. PGC-1 $\alpha$ deficiency causes multi-system energy metabolic derangements: muscle dysfunction, abnormal weight control and hepatic steatosis. PLoS Biology. 2005;3(4, article e101) doi: 10.1371/journal.pbio.0030101.

27. Qin W., Haroutunian V., Katsel P., et al. PGC-1 $\alpha$ expression decreases in the Alzheimer disease brain as a function of dementia. Archives of Neurology. 2009;66(3):352-361. doi: 10.1001/archneurol.2008.588.

28. Bartley A. F., Lucas E. K., Brady L. J., et al. Interneuron transcriptional dysregulation causes frequency-dependent alterations in the balance of inhibition and excitation in hippocampus. The Journal of Neuroscience. 2015;35(46):15276-15290. doi: 10.1523/JNEUROSCI.1834-15.2015.

29. Rubenstein J. L., Merzenich M. M. Model of autism: increased ratio of excitation/inhibition in key neural systems. Genes, Brain, and Behavior. 2003;2(5):255-267. doi: 10.1034/j.1601-183X.2003.00037.x.

30. Strekalova T., Evans M., Costa-Nunes J., et al. Tlr4 upregulation in the brain accompanies depression- and anxiety-like behaviors induced by a highcholesterol diet. Brain, Behavior, and Immunity. 2015;48:42-47. doi: 10.1016/j.bbi.2015.02.015.

31. Strekalova T., Costa-Nunes J. P., Veniaminova E., et al. Insulin receptor sensitizer, dicholine succinate, prevents both Toll-like receptor 4 (TLR4) upregulation and affective changes induced by a high-cholesterol diet in mice. Journal of Affective Disorders. 2016;196:109-116.

doi: $10.1016 /$ j.jad.2016.02.045.

32. Veniaminova E., Cespuglio R., Markova N., et al. Behavioral features of mice fed with a cholesterol-enriched diet: deficient novelty exploration and 
unaltered aggressive behavior. Translational Neuroscience and Clinics. 2016;2(2):87-95. doi: 10.18679/CN11-6030/R.2016.014.

33. Deacon R. M., Croucher A., Rawlins J. N. Hippocampal cytotoxic lesion effects on species-typical behaviours in mice. Behavioural Brain Research. 2002;132(2):203-213. doi: 10.1016/S0166-4328(01)00401-6.

34. Strekalova T., Steinbusch H. W. Measuring behavior in mice with chronic stress depression paradigm. Progress in Neuro-Psychopharmacology \& Biological Psychiatry. 2010;34(2):348-361.

35. Malatynska E., Steinbusch H. W., Redkozubova O., et al. Anhedonic-like traits and lack of affective deficits in 18-month-old C57BL/6 mice: implications for modeling elderly depression. Experimental Gerontology. 2012;47(8):552-564. doi: 10.1016/j.exger.2012.04.010.

36. Couch Y., Anthony D. C., Dolgov O., et al. Microglial activation, increased TNF and SERT expression in the prefrontal cortex define stressaltered behaviour in mice susceptible to anhedonia. Brain, Behavior, and Immunity. 2013;29:136-146. doi: 10.1016/j.bbi.2012.12.017.

37. Mantella R. C., Vollmer R. R., Amico J. A. Corticosterone release is heightened in food or water deprived oxytocin deficient male mice. Brain Research. 2005;1058(1-2):56-61. doi: 10.1016/j.brainres.2005.07.062.

38. Arakawa H., Blanchard D. C., Blanchard R. J. Central oxytocin regulates social familiarity and scent marking behavior that involves amicable odour signals between male mice. Physiology \& Behavior. 2015;146:36-46. doi: 10.1016/j.physbeh.2015.04.016.

39. Hahn M. E. Genetic "artifacts" an aggressive behavior. In: Simmel E. C., Hahn M. E., Waiters J. K., editors. Aggressive Behavior: Genetic and Neural Approaches. Hillsdale, NJ: Lawrence Erlbaum Associates; 1983.

40. Couch Y., Trofimov A., Markova N., et al. Low-dose lipopolysaccharide (LPS) inhibits aggressive and augments depressive behaviours in a chronic mild stress model in mice. Journal of Neuroinflammation. 2016;13(1):p. 108. doi: 10.1186/s12974-016-0572-0.

41. Strekalova T., Zörner B., Zacher C., Sadovska G., Herdegen T., Gass P. Memory retrieval after contextual fear conditioning induces c-Fos and JunB expression in CA1 hippocampus. Genes, Brain, and Behavior. 2003;2(1):310. doi: 10.1034/j.1601-183X.2003.00001.x.

42. Vignisse J., Steinbusch H. W., Grigoriev V., et al. Concomitant manipulation of murine NMDA- and AMPA-receptors to produce procognitive drug effects in mice. European Neuropsychopharmacology. 2014;24(2):309-320. doi: 10.1016/j.euroneuro.2013.06.010. 
43. Koolhaas J. M., Korte S. M., De Boer S. F., et al. Coping styles in animals: current status in behavior and stress-physiology. Neuroscience and Biobehavioral Reviews. 1999;23(7):925-993.

doi: 10.1016/S0149-7634(99)00026-3.

44. Krishna S., Keralapurath M. M., Lin Z., et al. Neurochemical and electrophysiological deficits in the ventral hippocampus and selective behavioral alterations caused by high-fat diet in female C57BL/6 mice. Neuroscience. 2015;297:170-181. doi: 10.1016/j.neuroscience.2015.03.068.

45. Koizumi H., Hashimoto K., Iyo M. Dietary restriction changes behaviours in brain-derived neurotrophic factor heterozygous mice: role of serotonergic system. The European Journal of Neuroscience. 2006;24(8):2335-2344. doi: 10.1111/j.1460-9568.2006.05094.x.

46. Pérez-Ortiz J. M., Galiana-Simal A., Salas E., González-Martín C., García-Rojo M., Alguacil L. F. A high-fat diet combined with food deprivation increases food seeking and the expression of candidate biomarkers of addiction. Addiction Biology. 2016:12-19. doi: 10.1111/adb.12389.

47. Vucetic Z., Carlin J. L., Totoki K., Reyes T. M. Epigenetic dysregulation of the dopamine system in diet-induced obesity. Journal of Neurochemistry. 2012;120(6):891-898. doi: 10.1111/j.1471-4159.2012.07649.x.

48. Grissom N. M., George R., Reyes T. M. The hypothalamic transcriptional response to stress is severely impaired in offspring exposed to adverse nutrition during gestation. Neuroscience. 2015;342:200-211. doi: 10.1089/ham.2016.0142.

49. South T., Huang X. F. Temporal and site-specific brain alterations in $\mathrm{CB} 1$ receptor binding in high fat diet-induced obesity in $\mathrm{C} 57 \mathrm{Bl} / 6$ mice. Journal of Neuroendocrinology. 2008;20(11):1288-1294. doi: 10.1111/j.13652826.2008.01785.x.

50. Labouesse M. A., Stadlbauer U., Langhans W., Meyer U. Chronic high fat diet consumption impairs sensorimotor gating in mice. Psychoneuroendocrinology. 2013;38(11):2562-2574.

doi: 10.1016/j.psyneuen.2013.06.003.

51. Grissom N. M., Reyes T. M. Gestational overgrowth and undergrowth affect neurodevelopment: similarities and differences from behavior to epigenetics. International Journal of Developmental Neuroscience. 2013;31(6):406-414. doi: 10.1016/j.ijdevneu.2012.11.006.

52. Kaczmarczyk M. M., Machaj A. S., Chiu G. S., et al. Methylphenidate prevents high-fat diet (HFD)-induced learning/memory impairment in 
juvenile mice. Psychoneuroendocrinology. 2013;38(9):1553-1564. doi: 10.1016/j.psyneuen.2013.01.004.

53. Sobesky J. L., Barrientos R. M., De May H. S., et al. High-fat diet consumption disrupts memory and primes elevations in hippocampal IL-1 $\beta$, an effect that can be prevented with dietary reversal or IL-1 receptor antagonism. Brain, Behavior, and Immunity. 2014;42:22-32. doi: 10.1016/j.bbi.2014.06.017.

54. Kang S. S., Jeraldo P. R., Kurti A., et al. Diet and exercise orthogonally alter the gut microbiome and reveal independent associations with anxiety and cognition. Molecular Neurodegeneration. 2014;9(1):p. 36. doi: 10.1186/1750-1326-9-36.

55. Heyward F. D., Walton R. G., Carle M. S., Coleman M. A., Garvey W. T., Sweatt J. D. Adult mice maintained on a high-fat diet exhibit object location memory deficits and reduced hippocampal SIRT1 gene expression. Neurobiology of Learning and Memory. 2012;98(1):25-32. doi: 10.1016/j.nlm.2012.04.005.

56. Deacon R. M. Burrowing in rodents: a sensitive method for detecting behavioral dysfunction. Nature Protocols. 2006;1(1):118-121. doi: 10.1038/nprot.2006.19.

57. Teeling J. L., Felton L. M., Deacon R. M., Cunningham C., Rawlins J. N., Perry V. H. Sub-pyrogenic systemic inflammation impacts on brain and behavior, independent of cytokines. Brain, Behavior, and Immunity. 2007;21(6):836-850. doi: 10.1016/j.bbi.2007.01.012.

58. Richman D. M., Barnard-Brak L., Bosch A., Thompson S., Grubb L., Abby L. Predictors of self-injurious behaviour exhibited by individuals with autism spectrum disorder. Journal of Intellectual Disability Research. 2013;57(5):429-439. doi: 10.1111/j.1365-2788.2012.01628.x.

59. Kalaivanisailaja J., Manju V., Nalini N. Lipid profile in mice fed a highfat diet after exogenous leptin administration. Polish Journal of Pharmacology. 2003;55(5):763-769.

60. Fujita Y., Maki K. High-fat diet-induced obesity triggers alveolar bone loss and spontaneous periodontal disease in growing mice. BMC Obesity. 2016;3(1):p. 1. doi: 10.1186/s40608-016-0082-8.

61. Milanski M., Arruda A. P., Coope A., et al. Inhibition of hypothalamic inflammation reverses diet-induced insulin resistance in the liver. Diabetes. 2012;61(6):1455-1462. doi: 10.2337/db11-0390. 
62. Rossignol D. A., Frye R. E. Mitochondrial dysfunction in autism spectrum disorders: a systematic review and meta-analysis. Molecular Psychiatry. 2012;17(3):290-314. doi: 10.1038/mp.2010.136.

63. Herbert M. R., Buckley J. A. Autism and dietary therapy: case report and review of the literature. Journal of Child Neurology. 2013;28(8):975-982. doi: $10.1177 / 0883073813488668$.

64. Gorelick P. B. Role of inflammation in cognitive impairment: results of observational epidemiological studies and clinical trials. Annals of the New York Academy of Sciences. 2010;1207(1):155-162. doi: 10.1111/j.17496632.2010.05726.x.

65. Engelhart M. J., Geerlings M. I., Meijer J., et al. Inflammatory proteins in plasma and the risk of dementia: the rotterdam study. Archives of Neurology. 2004;61(5):668-672. doi: 10.1001/archneur.61.5.668.

66. Nguyen J. C. D., Killcross A. S., Jenkins T. A. Obesity and cognitive decline: role of inflammation and vascular changes. Frontiers in Neuroscience. 2014;8:p. 375. doi: 10.3389/fnins.2014.00375.

67. Napoli E., Ross-Inta C., Wong S., et al. Mitochondrial dysfunction in pten haplo-insufficient mice with social deficits and repetitive behavior: interplay between Pten and p53. PloS One. 2012;7(8, article e42504) doi: 10.1371/journal.pone.0041316.

68. Mancuso M., Calsolaro V., Orsucci D., et al. Mitochondria, cognitive impairment, and Alzheimer's disease. International Journal of Alzheimer's Disease. 2009;2009:8. doi: 10.4061/2009/951548.951548

69. Ruhl T., Jonas A., Seidel N. I., et al. Oxidation and cognitive impairment in the aging zebrafish. Gerontology. 2015;62(1):47-57. doi: $10.1159 / 000433534$.

70. Giugliano D., Ceriello A., Esposito K. The effects of diet on inflammation: emphasis on the metabolic syndrome. Journal of the American College of Cardiology. 2006;48(4):677-685.

71. Graham L. C., Harder J. M., Soto I., de Vries W. N., John S. W. M., Howella G. R. Chronic consumption of a western diet induces robust glial activation in aging mice and in a mouse model of Alzheimer's disease. Scientific Reports. 2016;6:p. 21568. doi: 10.1038/srep21568.

72. Farooqui A. A. Inflammation and Oxidative Stress in Neurological Disorders. Effect of Lifestyle, Genes, and Age. Chapter: The Effects of Diet, Exercise, and Sleep on Brain Metabolism and Function. Switzerland: Springer International Publishing; 2014. 
73. Neves F. A., Cortez E., Bernardo A. F., et al. Heart energy metabolism impairment in Western-diet induced obese mice. The Journal of Nutritional Biochemistry. 2014;25(1):50-57. doi: 10.1016/j.jnutbio.2013.08.014.

74. Pomytkin I. A., Cline B. H., Anthony D. C., Steinbusch H. W., Lesch K. P., Strekalova T. Endotoxaemia resulting from decreased serotonin tranporter (5-HTT) function: a reciprocal risk factor for depression and insulin resistance? Behavioural Brain Research. 2015;276:111-117. doi: 10.1016/j.bbr.2014.04.049.

75. Herzig S., Long F., Jhala U. S., et al. CREB regulates hepatic gluconeogenesis through the coactivator PGC-1. Nature. 2001;413(6852):179183. doi: $10.1038 / 35093131$.

76. Heyward F. D., Gilliam D., Coleman M. A., et al. Obesity weighs down memory through a mechanism involving the neuroepigenetic dysregulation of Sirt1. The Journal of Neuroscience. 2016;36(4):1324-1335. doi: 10.1523/JNEUROSCI.1934-15.2016.

77. Larkina T. A., Sazanova A. L., Fomichev K. A., et al. HMG1A and PPARG are differently expressed in the liver of fat and lean broilers. Journal of Applied Genetics. 2011;52(2):225-228. doi: 10.1007/s13353-010-0023-z. 


\section{Supplementary Data}

Table 1. The composition of the diets with respect to the content of carbohydrates, saturated / unsaturated fat and protein, $w / w$

\begin{tabular}{|l|l|l|}
\hline & Standard chow, \% & Western diet, \% \\
\hline Total fat & 5 & 21 \\
\hline Saturated fat & 1 & 20 \\
\hline $\begin{array}{l}\text { Polyunsaturated and } \\
\text { monounsaturated fat }\end{array}$ & 4 & 1 \\
\hline $\begin{array}{l}\text { Carbohydrates: starch } \\
\text { and sugars }\end{array}$ & 61 & 50 \\
\hline Protein & 24 & 19 \\
\hline Fiber & 5 & 5 \\
\hline Cholesterol & 0 & 0.2 \\
\hline
\end{tabular}

Table 2. Sequences of primers used

\begin{tabular}{|l|l|l|}
\hline $\begin{array}{l}\text { mRNA } \\
\text { target }\end{array}$ & \multicolumn{2}{|l|}{ Primer sequence $\left(5^{\prime} \rightarrow 3^{\prime}\right)$} \\
\hline PPARGC1a & Forward & CTCCAGTTCCGGCTCCTC \\
\cline { 2 - 3 } & Reverse & CCCTGTGCTCTCACGTCTG \\
\hline Gapdh & Forward & ACCCCTTCATTGACCTCAACTACATG \\
\cline { 2 - 3 } & Reverse & CCTTCTCCATGGTGGTGAAGAC \\
\hline
\end{tabular}




\section{Chapter 3}

\section{Prefrontal cortex}

inflammation and liver pathologies accompany cognitive and motor deficits following Western diet consumption in non-obese female mice 


\section{Chapter 3. Prefrontal cortex inflammation and liver pathologies accompany cognitive and motor deficits following Western diet consumption in non-obese female mice}

Ekaterina Veniaminova ${ }^{\mathrm{a}, \mathrm{b}}$, Margarita Oplatchikova ${ }^{\mathrm{b}}$, Lucien Bettendorffc, Elena Kotenkovad, Alexander Lysko ${ }^{\mathrm{e}}$, Ekaterina Vasilevskaya $^{\mathrm{d}}$, Allan V. Kalueff,g,h, Liliya Fedulovad, Aleksei Umriukhin $^{\mathrm{b}}$, Klaus-Peter Lescha,b,i, Daniel C. Anthony ${ }^{\mathrm{b}, \mathrm{j}}$, Tatyana Strekalova ${ }^{a, b, i}$

aDepartment of Psychiatry and Neuropsychology, School for Mental Health and Neuroscience, Maastricht University, Universiteitssingel 40, NL 6229ER Maastricht, the Netherlands; bLaboratory of Psychiatric Neurobiology, Institute of Molecular Medicine and Department of Normal Physiology, Sechenov First Moscow State Medical University, Trubetskaya Str. 8, 119991 Moscow, Russia; 'Laboratory of Neurophysiology, GIGA-Neurosciences, University of Liège, Av. Hippocrate 15, 4000 Liège, Belgium; dV.M. Gorbatov Federal Research Center for Food Systems of RAS, Tallalikhina Str. 26, 109316 Moscow, Russia; eInstitute of General Pathology and Pathophysiology, Baltiyskaya Str. 8, 125315 Moscow, Russia; fSchool of Pharmacy, Southwest University, 400715 Chongqing, China; sInstitute of Translational Biomedicine, St. Petersburg State University, Universitetskaya Nab. 7-9, 199034 St. Petersburg, Russia; ${ }^{\text {h} U r a l ~ F e d e r a l ~ U n i v e r s i t y, ~ M i r a ~ S t r . ~ 19, ~} 620002$ Ekaterinburg, Russia; i Division of Molecular Psychiatry, Laboratory of Translational Neuroscience, Center of Mental Health, University of Würzburg, Margarete-Höppel-Platz 1, 97080, Würzburg, Germany; jDepartment of Pharmacology, Oxford University, Mansfield Road, OX1 3QT Oxford, UK

Veniaminova E, Oplatchikova $M$, Bettendorff L, Kotenkova E, Lysko A, Vasilevskaya E, Kalueff AV, Fedulova L, Umriukhin A, Lesch KP, Anthony DC, Strekalova T. Prefrontal cortex inflammation and liver pathologies accompany cognitive and motor deficits following Western diet consumption in non-obese female mice. Life Sciences. 2020;241: 117163. 


\section{Abstract}

\section{Aims}

The high sugar and lipid content of the Western diet (WD) is associated with metabolic dysfunction, non-alcoholic steatohepatitis, and it is an established risk factor for neuropsychiatric disorders. Our previous studies reported negative effects of the WD on rodent emotionality, impulsivity, and sociability in adulthood. Here, we investigated the effect of the WD on motor coordination, novelty recognition, and affective behavior in mice as well as molecular and cellular endpoints in brain and peripheral tissues.

\section{Main methods}

Female C57BL/6 J mice were fed the WD for three weeks and were investigated for glucose tolerance, insulin resistance, liver steatosis, and changes in motor coordination, object recognition, and despair behavior in the swim test. Lipids and liver injury markers, including aspartate-transaminase, alanine-transaminase and urea were measured in blood. Serotonin transporter (SERT) expression, the density of Iba1-positive cells and concentration of malondialdehyde were measured in brain.

\section{Key findings}

WD-fed mice exhibited impaired glucose tolerance and insulin resistance, a loss of motor coordination, deficits in novel object 
exploration and recognition, increased helplessness, dyslipidemia, as well as signs of a non-alcoholic steatohepatitis (NASH)-like syndrome: liver steatosis and increased liver injury markers. Importantly, these changes were accompanied by decreased SERT expression, elevated numbers of microglia cells and malondialdehyde levels in, and restricted to, the prefrontal cortex.

\section{Significance}

The WD induces a spectrum of behaviors that are more reminiscent of ADHD and ASD than previously recognized and suggests that, in addition to the impairment of impulsivity and sociability, the consumption of a WD might be expected to exacerbate motor dysfunction that is also known to be associated with adult ADHD and ASD.

\section{Keywords}

Western diet, Non-alcoholic steatohepatitis (NASH), Motor coordination, Cognitive deficits, Microglia, Oxidative stress, Prefrontal cortex, Attention Deficit Hyperactivity Disorder (ADHD), Autism Spectrum Disorder (ASD), Mice 


\section{Introduction}

The "Western diet" (WD) is characterized by high intake of saturated fats, cholesterol and refined sugars, and is recognized as a serious risk factor for a number of medical conditions [1,2], including neuropsychiatric disorders [3-5]. The effect of a WD on mood, cognition and associated psychiatric syndromes, including autism spectrum disorder (ASD) [6,7] and attention-deficit/hyperactivity disorder (ADHD) [8], has been most extensively studied during development or in the early postnatally period. However, recent studies, including our own, have revealed that the WD can also induce changes in mood and cognition in adult mice that are reminiscent of the symptoms that are characteristic of ASD and ADHD [9-13].

Mice fed the WD for three weeks exhibit open field hyperactivity, increased impulsivity, anxiety and depression-like behaviors, and altered social interactions [11-14]. These characteristics overlap with the ASD and ADHD syndromes [15-17]. It is of interest to note that ASD and ADHD in adolescents and young adults are frequently comorbid with metabolic syndrome and diabetes [18-20]. The WD has also been shown to modify motor coordination both in humans and in mice given the WD for extended periods [9,21,22]. Attentional control to engage motor/cognitive tasks involves the prefrontal cortex [23], where decreased activity within this part of the brain is argued to underpin ADHD [24] and ASD [25]. We hypothesized that the WD 
regime might induce an $\mathrm{ADHD} / \mathrm{ASD}$-like syndrome, which would also include deficits in motor coordination, and would be associated with cellular and molecular changes the prefrontal cortex. As a prolonged dietary challenge with a WD induces widespread changes in the brain [26], we employed a short dietary regime in the hope that we might reveal early regional vulnerabilities that could provide insight into the sites that are responsible for the diet-induced behavioral changes.

Female mice display a greater vulnerability to negative effects of the WD [27]. For example, female C57BL6J mice fed the WD for three weeks exhibited a more marked liver steatosis, as shown by increased level of liver triglycerides, as well as increased levels of inflammatory markers in the liver, such as increased expression of CD68, TNF and CCL2, than male mice.

Contrastingly, male mice exhibit greater dyslipidaemia, displaying increased level of triglycerides and decreased level of nonesterified fatty acids in blood and weight gain, which is not observed in female mice. While there is a higher prevalence of males with ASD than females, it would be incorrect to suggest that $\mathrm{ASD} / \mathrm{ADHD}$ is restricted to males, thus, the use of female mice remains a valid approach. In adulthood, the overall prevalence of adult ADHD is $4.4 \%$, and the figure is, higher for males (5.4\%) than for females $(3.2 \%)$, but a conservative estimate still reveals that 124 million women worldwide live with ADHD. For ASD, the male/female ratio may be as high as 
4:1 and discrete mechanisms may exist that confer "resilience" in women, but ASD prevalence in the population is estimated to be between $2.3 \%$ and $9.9 \%$ [28] and thus the number of women affected is still very high compared to most other pathologies. Hence, there is certainly a need to understand the impact of the WD in females as the number of women by these conditions worldwide is enormous.

In the present experiments, we also investigated the impact of the WD on glucose and insulin tolerance, liver steatosis, serum concentrations of lipids, urea and liver injury markers. Microglial activation in the hippocampus, prefrontal and motor cortex were evaluated. Malondialdehyde concentration, an oxidative stress marker, was measured in prefrontal cortex, hippocampus, hypothalamus and dorsal raphe. Serotonin transporter (SERT) expression, which is decreased by the WD, obesity [29-31], and ADHD [32,33] was also evaluated in the prefrontal cortex and hippocampus.

\section{Methods}

\subsection{Animals}

Experiments were performed using 3-month-old female C57BL/6J mice obtained from certified Charles River provider IPAC RAS, Moscow region. Mice were housed 2-3 per cage during the study, under a 12 h light-dark cycle (lights on: 21:00 h) with food and water ad libitum and under controllable laboratory conditions $\left(22 \pm 1{ }^{\circ} \mathrm{C}\right.$, 
$55 \%$ humidity). Laboratory housing conditions and experimental procedures were set up and maintained in accordance with the European Communities Council Directive for the care and use of laboratory animals (2010/63/EU) and approved by the local ethics committee (Ethics Committee of Sechenov First MSMU, \#11-18).

\subsection{Study design and diets}

3-month-old female C57BL/6 J mice were fed with a standard laboratory diet (control diet, CD) with an energy content of $3.2 \mathrm{kcal} / \mathrm{g}$ and $3.3 \%$ of fat $(0.6 \%$ of saturated fat) (V1534-000, Ssniff Spezialdiäten GmbH, Soest, Germany) or with a diet containing $0.2 \%$ cholesterol, $21 \%$ of fat ( $9 \%$ of saturated fat), and an energy content of $4.6 \mathrm{kcal} / \mathrm{g}$, 'Western diet' (WD), (D12079B, Research Diet Inc., New Brunswick, NJ, USA, Supplementary Table 1) for three weeks as described elsewhere [11-13]. Body weight, and food and water intake were monitored daily during the first 4 days of dietary challenge and each 3rd day thereafter. The experiment was carried out in three runs, 6-7 mice per group were used in each run (Fig. 1).

After the three-week dietary challenge, mice from cohort 1 were studied for depressive-like behavior in the two-day swim test (days 21 and 22) and a glucose tolerance test (day 23) and were culled on day 24. Their blood and liver were collected for biochemical analysis and histological assessment. Spleen, kidney, muscle gastrocnemius, heart, 
abdominal fat were harvested and weighed; the brain was dissected and the hippocampus and prefrontal cortex were collected for analysis of SERT gene expression. Dietary and control mice from cohort 2 were studied for muscle strength using the inverted screen test and weights test on day 19, in motor tests: rotarod, wire test and pole tests on Days 20-21, followed by a glucose tolerance test on day 22 .

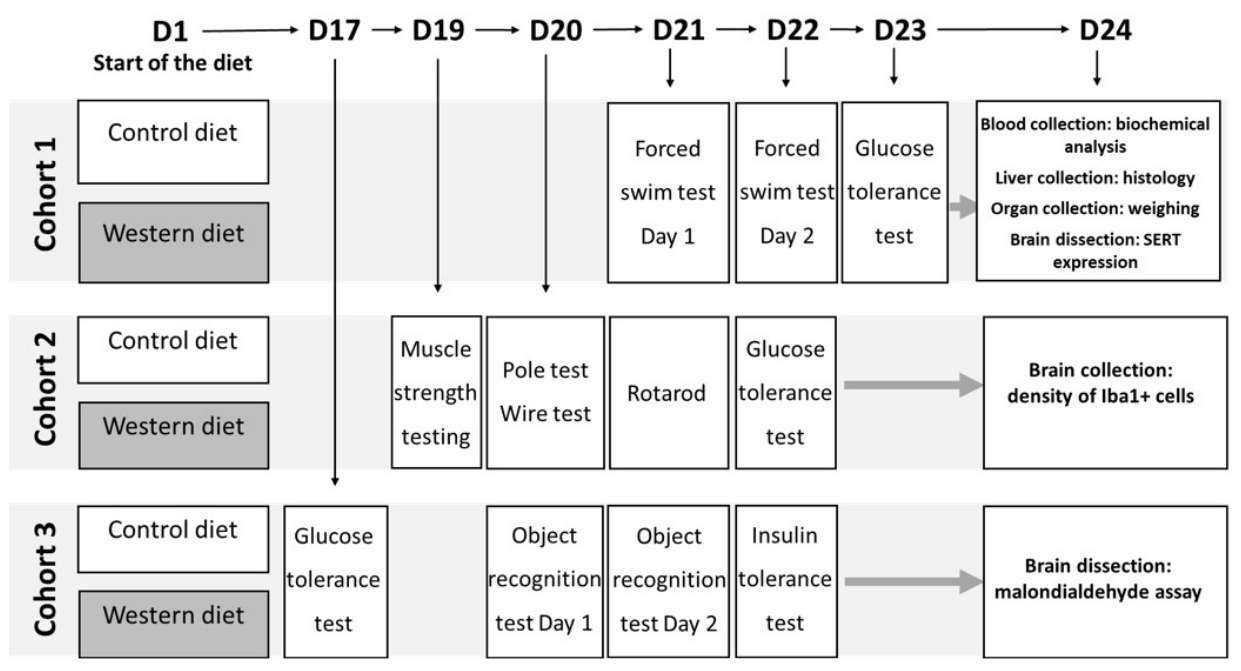

Fig. 1. Experiment design. Three cohorts of mice were fed either control or WD for 3 weeks. Thereafter, mice from cohort 1 were studied in the two-day forced swim test, glucose tolerance test and were culled. Their blood and liver were collected for the biochemical blood analysis and histological assessment of lipid accumulation; spleen, kidney, heart, and abdominal fat were harvested, brain was dissected for SERT expression measurement. Mice from cohort 2 were studied for muscle strength in the Weights test and inverted screen, and in motor tests: rotarod, wire test and pole test, and glucose tolerance test. Thereafter animals were culled and their brains were dissected for Iba1-staining. Mice from cohort 3 were tested for glucose tolerance on day 17, and thereafter for object exploration/recognition, and insulin resistance. Mice were culled and their brains were dissected for the measurement of the malondialdehyde concentration in the prefrontal cortex, hippocampus, dorsal raphe and hypothalamus. 
Thereafter, the animals were culled and their brains were collected for Iba1 immunohistochemistry. Mice from cohort 3 were tested for glucose tolerance on day 17, object exploration / recognition, on days 20-21 and insulin tolerance test on day 22 .

In order to prevent potential confounds owing to serial behavioral tests in the mice, and to avoid the effects of long interruptions in diet intake during the glucose-tolerance and insulin-tolerance tests, we extended the duration of our battery of behavioral tests to either side of the 21-day-long period of dietary intervention. As such, the onset and the end of testing was extended by 1-2 days. Thus, a small increase in the total duration of the experimental period was necessary to compensate for the periods of food deprivation during the glucose and insulin tolerance tests, in order to comply with the original 21-day long feeding protocol [27]. These adjustments were also employed in our previous studies and were shown to have no effect on the outcomes, as cognitive and emotional aberrations in WD-challenged mice were observed on days 20 and 21 of the dietary challenge [10-13]. Mice were culled and their brains were dissected for the measurement of the concentration of malondialdehyde (MDA), oxidative stress marker, in the prefrontal cortex, hippocampus, dorsal raphe and hypothalamus.

\subsection{Behavioral testing}

Behavioral tests were carried out during an active period of the animals' light cycle (09:00-21:00) and analyzed offline by the 
experimenter who was unaware of diet each animal had received. Behavioral equipment was thoroughly cleaned with water between each test.

\subsubsection{Rotarod}

Motor coordination was studied in seven consequent trials of nonaccelerating rotarod as described elsewhere [34]. As previous experiments revealed the occurrence of motor abnormalities in the mice housed on a WD in the rotarod test at day 19, (Veniaminova, unpublished data; Supplementary Fig. S1), here began testing the mice for abnormal motor functions from day 19. Rotarod set-up consisted of a cylinder with a diameter of $3.5 \mathrm{~cm}$, which rotated at a constant speed (10 rpm). The animals were first trained to stay on the rotating rod for as long as they could over 6 trials. The maximum latency of falling was set at $10 \mathrm{~min}$. If a mouse successfully completed the task it was not subjected to further trials and the latency for all the further trials was set as $10 \mathrm{~min}$. Then all the mice participated in final 7 th trial. The learning curve was drawn and the total time that an animal could stay on the rod before falling in the 7th trial was used as a measure of the task acquisition.

\subsubsection{Wire test}

The wire hanging test is based on the instinct of a mouse to avoid falling. Mice were allowed to grip a horizontally hanging wire (diameter $0.3 \mathrm{~cm}$, height above surface $60 \mathrm{~cm}$ ) with four limbs [34]. Their ability 
to keep a balance on a wire and grip was measured by scoring a latency to fall. For each mouse the best result of two trials was analyzed.

\subsubsection{Pole test}

Mice were placed on top of a vertically standing bar (diameter $1.1 \mathrm{~cm}$, height $60 \mathrm{~cm}$ ) and allowed to climb down to a horizontal surface [34]. The latency to reach the ground with all four paws, events of jumping, sliding and falling were scored. For each mouse the best result of the two trials was analyzed.

\subsubsection{Weights test}

The 'Weights' test was used to assess muscular strength and was performed as described elsewhere [35]. Each weight consisted of a ball of tangled fine gauge stainless steel wire $(7 \mathrm{~g})$ attached to a series of steel chain links, each weighing 13 g. The number of links ranged from one to seven. The mouse was held by the base of the tail and allowed to grasp the first weight $(20 \mathrm{~g})$ which was lying on the laboratory bench. As it grasped the wire scale collector with its forepaws it was raised until the link was clear of the bench. A hold of three seconds was used as a criterion. If the mouse dropped the weight in $<3 \mathrm{~s}$, the time it held the weight was noted. The mouse was given three chances to hold the weight for $3 \mathrm{~s}$. If it failed three times the mouse was assigned the maximum time/weight achieved. If it held the weight for $3 \mathrm{~s}$ then the next heaviest weight was tried. A final total score was calculated as the product of the number of links in the heaviest chain 
held for the full $3 \mathrm{~s}$, multiplied by the time (sec) it is held. If the heaviest weight was dropped before $3 \mathrm{~s}$ an appropriate intermediate value was calculated.

\subsubsection{Inverted screen test}

Inverted screen test was used to test muscle strength according to published protocols [35,36]. A $43 \mathrm{~cm}$ square of wire mesh consisting of $12 \mathrm{~mm}$ squares of $1 \mathrm{~mm}$ diameter wire was surrounded by a $4 \mathrm{~cm}$ deep wooden beading (which prevented the mouse from climbing on to the other side). The mouse was placed in the center of the wire mesh screen, the screen was rotated to an inverted position over $2 \mathrm{~s}$, with the mouse's head declining first and hold steadily $50 \mathrm{~cm}$ above a padded surface. Latency to fall off was used as a measure of muscle strength. $180 \mathrm{~s}$ was set as the maximum latency.

\subsubsection{Object exploration/recognition test}

Mice were studied for new object exploration / recognition in a 2-day test, as described elsewhere [37]. The apparatus for the object exploration / recognition test consisted of a plastic cage $(21 \times 27 \times 14 \mathrm{~cm})$ with opaque walls and two objects ("brush" and "flower", $7 \times 4 \times 3 \mathrm{~cm}$, attached to $15 \mathrm{ml}$ tube, Supplementary Fig.S2) placed symmetrically

$2 \mathrm{~cm}$ away from the cage wall in the opposite corners of the cage. The lightning of $5 \mathrm{~lx}$ intensity was used. Objects used in the new object recognition test were either disposable and were new for each mouse (flowers were made from paper) or changeable and washable with water 
and mild detergent (plastic brush), so that any contamination (the smell of a preceding mouse) was virtually excluded. This methodology has been extensively validated in previous studies and has been successfully employed in different paradigms [37,38].

On the day 1 of the test, two identical objects were used, and a mouse was placed in the cage at an equal distance from the objects and allowed to explore the area freely for $15 \mathrm{~min}$. On the day 2, one object was replaced by the new object and mouse was placed in the cage for $15 \mathrm{~min}$. The duration of object exploration, which was defined as the mouse's nose being directed towards the object while it is situated at a distance $<2 \mathrm{~cm}$ from the object, was scored off-line for each object and both objects combined. A percentage of time of exploration for the "new object", that has replaced the "familiar object" from Day 1 to Day 2, over the total duration of exploration was compared against $50 \%$-chance level of approaching either object and was taken as a parameter of object recognition memory.

\subsubsection{Swim test}

The test was performed as described elsewhere [11,12,39]. Mice were placed into a plastic transparent cylinder $(\varnothing 17 \mathrm{~cm})$ filled with water $\left(+23{ }^{\circ} \mathrm{C}\right.$, water height $13 \mathrm{~cm}$, cylinder height $\left.20 \mathrm{~cm}\right)$ under the red lightning for $6 \mathrm{~min}$. The test was repeated with a $24 \mathrm{~h}$ interval. Duration of floating behavior, a sign of "despair" and helplessness, defined by the absence of any directed movements of animals' head 
and body, was scored offline for 2-min intervals and for the whole test period. Latency to float was evaluated as well. Additionally, the total duration of climbing behavior, defined as quick movements of the forelimbs causing the front paws break the surface of the water near the cylinder wall, and active swimming, defined as movement of forelimbs or hind limbs in a paddling fashion, were scored. Behavioral protocol for the swim test was previously validated using imipramine [39-41].

\subsection{Glucose tolerance test}

Oral glucose tolerance test (OGTT) was performed according to a published protocol [13]. We sought to match, as closely as possible, the 21-day dietary exposure regime in accordance with the original description of the model [27]. The glucose tolerance and insulin tolerance tests require prolonged food deprivation, and, in order to avoid any confounding effects of the diet deprivation on the metabolic and molecular parameters, the duration of the study was increased so that actual dietary exposure would be 21-22 days for each cohort (see Supplementary file). The test mice were fasted overnight for $18 \mathrm{~h}$, beginning at 1600 . Thereafter, a glucose solution $(2 \mathrm{~g} / \mathrm{kg}, 1.8 \mathrm{~g} / \mathrm{l})$ was delivered by oral gavage and blood was sampled from the tail vein. Samples were obtained prior to glucose administration at time point 0 and 5, 15, 30, 60, 90 min afterwards. The level of blood glucose was 
analyzed using the OneTouch UltraEasy glucometer and strips (LifeScan OneTouch, Dubai, UAE). Blood glucose concentrations and the area under a curve (AUC) for the values normalized to baseline glucose levels, were analyzed.

\subsection{Insulin tolerance test}

Insulin tolerance test (ITT) was performed as previously described [42]. Before the test, mice underwent a $5 \mathrm{~h}$ morning fast that began at 1030. Thereafter, a human insulin solution $(0.25 \mathrm{IU} / \mathrm{kg}$, diluted in saline) was injected intraperitoneally and blood was sampled from the tail vein. Samples were obtained before insulin injection at time point 0 and 15, 30, 45 and 60 min afterwards. The level of blood glucose was analyzed using the OneTouch UltraEasy glucometer and strips (LifeScan OneTouch, Dubai, UAE). If, during this test, blood glucose levels dropped to below $20 \mathrm{mg} / \mathrm{dl}, 1 \mathrm{~g} / \mathrm{kg}$ glucose was administered to counteract the effects of insulin, in order to reduce animal suffering). Blood glucose concentrations and the area under a curve (AUC) for the values normalized to baseline glucose levels, were analyzed.

\subsection{Culling, blood and tissue collection}

Mice were terminally anaesthetized with isoflurane inhalation for a subsequent material collection. Blood was collected by cardiac puncture from mice from cohort 1 into ethylenediaminetetraacetic acid 
(EDTA)-coated tubes and centrifuged at $2260 \mathrm{~g}$ for $8 \mathrm{~min}$ at $4{ }^{\circ} \mathrm{C}$. Plasma was collected and stored at $-80{ }^{\circ} \mathrm{C}$ until use for biochemical analysis. Mice were then transcardially perfused with $10 \mathrm{ml}$ ice-cold saline, internal organs (liver, spleen, heart, left kidney, right kidney) and visceral fat were isolated and weighted. Relative weight was calculated as organ weight divided by body weight. The liver left medial lobe was immediately used for Oil Red O staining. Brain was dissected and prefrontal cortex and hippocampus were isolated and stored at $-80{ }^{\circ} \mathrm{C}$ until use for gene expression analysis. Mice from cohort 2 were perfused with $10 \mathrm{ml}$ ice-cold saline followed by $4 \%$ paraformaldehyde via left ventricle, brains were removed, post-fixed in PFA for $12 \mathrm{~h}$ and cryoprotected in 30\% sucrose for $12 \mathrm{~h}[43,44]$ and then embedded in a mold filled with OCT compound and snap-frozen in dry ice-cooled isopentane. Samples were stored at $-30{ }^{\circ} \mathrm{C}$ until use for immunohistochemical analysis. The right gastrocnemius muscles were isolated and weighted. Mice from cohort 3 were subjected after anesthesia to a perfusion of the left ventricle with $10 \mathrm{ml}$ ice-cold saline, their brains were isolated, prefrontal cortex, hippocampus, hypothalamus and dorsal raphe were dissected and stored at $-80{ }^{\circ} \mathrm{C}$ until use.

\subsection{Blood biochemical analysis}

Plasma concentrations of total cholesterol, triglyceride, low density lipoprotein (LDL) and high-density lipoprotein (HDL), glucose, 
aspartate transaminase (AST), alanine transaminase (ALT) and urea were measured using the semiautomatic analyzer BioChem SA and commercial kits (High Technology Inc., Walpole, MA, USA) according to the manufacturers' instructions. Remnant cholesterol level was calculated as the difference between total cholesterol, LDL and HDL cholesterols.

\subsection{Liver Oil Red O staining}

Oil Red O staining of the liver was performed according to a published protocol [45]. 10- $\mu$ m-thick sections of fresh frozen liver tissue were cut on a cryostat microtome Microm HM-525 (Carl Zeiss, Oberkochen, Germany). Sections were rinsed with $60 \%$ isopropanol, incubated in Oil Red O solution (Sigma-Aldrich) for 10 min and again rinsed with $60 \%$ isopropanol. Slides were coversliped using an aqueous mounting medium (Leika, Wetzlar, Germany). Staining was examined with a light microscope AxioImaiger A1 (Carl Zeiss) using image analysis system AxioVision 4.7.1.0 (Carl Zeiss) at 20× magnification. Two to four images per section and two liver sections per animal were analyzed for staining density using the ImageJ software. The density of Oil Red O staining was evaluated in WD-group in percent from mean values of controls and was used a measure of liver steatosis. 


\subsection{RNA extraction and quantitative reverse transcription PCR}

Total mRNA was extracted by using TRI Reagent (MRC, Cincinnati, OH, USA) according to the manufacturer instructions. Quantitative reverse transcription PCR (RT-qPCR) was carried out as described elsewhere $[13,46]$. Briefly, $1 \mu \mathrm{g}$ total RNA was converted into cDNA using random primers and Superscript III transcriptase (Invitrogen, Carlsbad, CA, USA). qRT-PCR was performed using the SYBR Green master mix (Bio-Rad Laboratories, Philadelphia, PA, USA) and the CFX96 Deep Well Real-Time PCR Detection System (Bio-Rad, Hercules, CA, USA). The sequences of the primers can be found in Supplementary Table 2. SERT expression was normalized to reference gene glyceraldehyde-3-phosphate dehydrogenase (GAPDH) mRNA and calculated as relative-fold changes compared to control mice as described elsewhere $[13,46]$.

\subsection{Immunohistochemical analysis of Iba1-positive cells in} the brain

Immunostaining with Iba1-antibody and the evaluation of the density of Iba1-positive cells in the prefrontal cortex, hippocampus and dorsal raphe were performed as described elsewhere [46]. Sagittal $10 \mu$ m-thick sections were cut on a cryostat microtome (Leica Biosystems, Wetzlar, Germany) and mounted on gelatin-coated slides. Slides were washed in PBS and blocked for non-specific protein binding 
with $10 \%$ goat serum in PBS for $1 \mathrm{~h}$. Then, sections were incubated in primary antibody (anti-Iba1, 1:2000, ab178846, AbCam, Cambridge, $\mathrm{UK}$ ) in $1 \%$ normal goat serum at $4{ }^{\circ} \mathrm{C}$ for $12 \mathrm{~h}$. Visualization was performed using biotinylated secondary antibodies in 1\% serum in PBS (Vector Laboratories, Peterborough UK) for $1 \mathrm{~h}$ at room temperature and a standard avidin-biotin amplification step (Vector Laboratories) followed by development in diaminobenzidine hydrochloride (DAB, $0.5 \mu \mathrm{g} / \mathrm{ml}$ ) in $0.1 \mathrm{M}$ phosphate buffer. Immunostaining was examined using a light microscope Leitz Dialux 20 (Leica, Wetzlar, Germany) and digital camera Basler ACE (Basler Group, Ahrensburg, Germany). Microvisioneer slide scanning software (GT Vision, UK) was used to visualize brain areas at $20 \times$ magnification. The area of prefrontal cortex was specifically delineated according to the Paxinos and Franklin's the Mouse brain in stereotaxic coordinates (lateral 0.12-0.36, Supplementary Fig.S3A), and Iba1-positive cells were counted within this. The hippocampus (lateral 0.60-1.20) and motor cortex (lateral 0.60-1.20) areas, which appeared on the same slides were also examined (Supplementary Figs. S3B,C). Cell counting was carried out using ImageJ software. Three sections per each structure per animal were analyzed. The density of Iba1-positive cells was calculated as number of Iba1-positive cells divided by examined area. 


\subsection{Malondialdehyde assay}

Concentrations of MDA were measured following Abcam ab118970 kit instructions (Abcam, Eugene, OR, USA). Briefly, the tissue was washed in cold PBS and homogenized in lysis solution, centrifuged at $13000 \mathrm{~g}$ for $10 \mathrm{~min}$. TBA reagent was added to a supernatant and incubated at $95{ }^{\circ} \mathrm{C}$ for $60 \mathrm{~min}$; the supernatant was analyzed at $532 \mathrm{~nm}$ in a 96-well microplate as described elsewhere [44].

\subsection{Statistics}

Data were analyzed using GraphPad Prism version 8.01 (San Diego, CA, USA). Repeated measurements were analyzed using a two-way ANOVA for repeated measures with Sidak's multiple comparisons test. For data with a normal distribution, unpaired t-tests were used, and where normality was not observed a Mann-Whitney test was employed. The level of significance was set at $\mathrm{P}<.05$. Data with a normal distribution were presented as Mean \pm SEM, and data that were not normally distributed were presented as Median or Median with interquartile range.

\section{Results}

\subsection{Physiological and metabolic effects of the Western diet}

The body weight of mice housed on WD did not differ from control group at any time point of the study across all cohorts 
(Supplementary Figs. S4AC), which is consistent with our previous findings [11-13]. No differences were found in the weight of the liver, kidneys, spleen, heart or visceral fat relative to total body mass (Supplementary Figs. S4DI). Daily intake of calories and water intake, normalized to body, weight did not differ between the groups (Supplementary Figs. S4JK). These data rule out uneven weight gain as a potential confound in the subsequent behavioral and molecular studies, which might have been expected to be a consequence of such a highly calorific diet.

In animals fed the WD for 21 days, a two-way ANOVA for repeated measures revealed a significant interaction between diet and the clearance of glucose from the blood over time in OGTT $(\mathrm{F}=5.245$, $\mathrm{P}=.0002$; Fig. 2A). Compared to control mice, the WD-fed group showed a significant increase in blood glucose levels at 10 and 15 min after the glucose bolus $(\mathrm{t}=4.131, \mathrm{P}=.0179$ and $\mathrm{t}=4.313, \mathrm{P}=.0144$, respectively, Sidak's multiple comparisons test). The AUC was significantly increased in the WD-fed group compared to the control group ( $\mathrm{t}=3.605, \mathrm{P}=.0082$, Unpaired t-test, Fig. 2B). In another cohort, glucose tolerance was also decreased on day 23 (Supplementary Fig. S5A). At baseline, blood glucose concentration did not differ between the groups that were subjected to $18 \mathrm{~h}$ of fasting prior to the OGTT (Supplementary Fig. S5B). These findings are also consistent with our previously reported results [12]. After 16 days of diet feeding, 
no difference in glucose tolerance was found between the groups (Supplementary Fig. S5C), supporting the importance of the 21-day feeding regime.

\section{Glucose tolerance test D22}

A

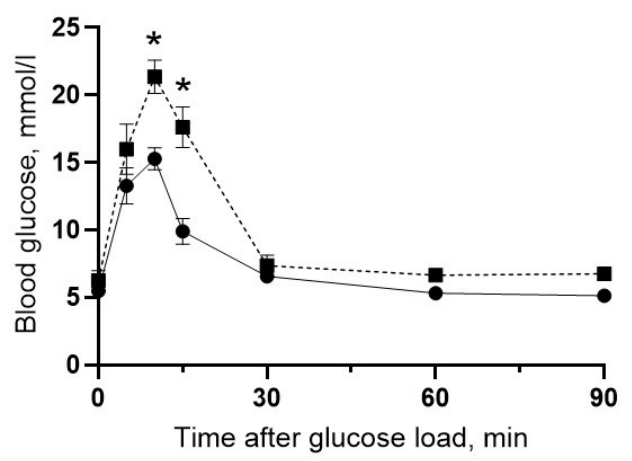

\section{Insulin tolerance test D22}

C
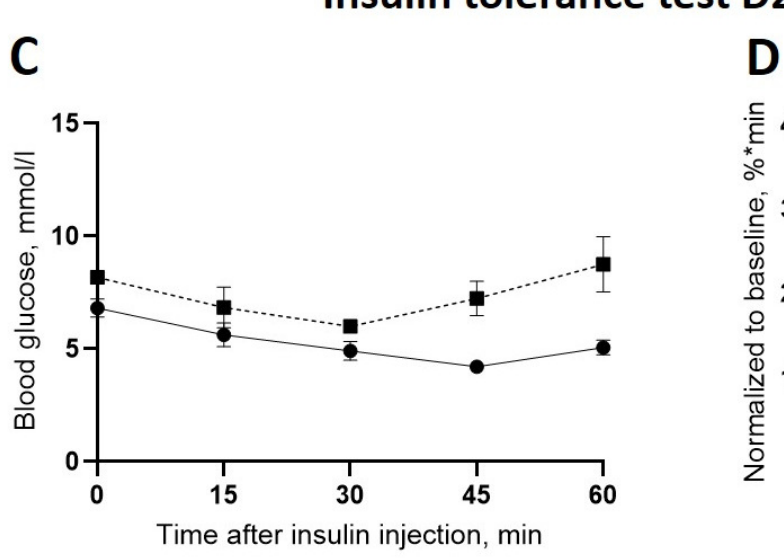

B

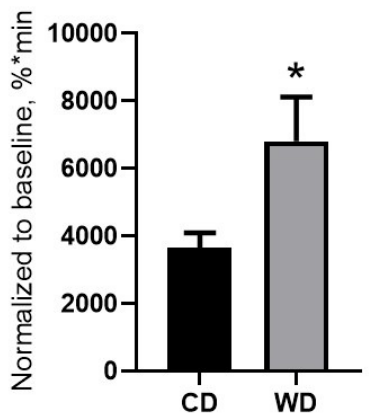

Fig. 2. Effects of housing on the Western diet on glucose tolerance and insulin resistance. Mice fed the Western diet for 3 weeks displayed (A-B) impaired glucose tolerance and (C-D) insulin tolerance. CD - Control diet, WD Western diet, AUC - area under the curve. ${ }^{*}$ - $\mathrm{P}<.05$ compared to CD (Unpaired t-test or Sidak's multiple comparisons test). Data are presented as Mean \pm SEM. 
A two-way ANOVA for repeated measures revealed a significant interaction between diet and time after insulin injection in the glucose level measured in the insulin tolerance test on day $22(\mathrm{~F}=5.802$, $\mathrm{P}=.0010$, Fig. 2C). The AUC for insulin tolerance was significantly increased in mice fed with the WD compared to controls $(\mathrm{t}=2.677$, $\mathrm{P}=.0363$, Unpaired t-test, Fig. 2D). At baseline, blood glucose concentration was increased in the animals fed WD compared to controls when animals were subjected to $5 \mathrm{~h}$ of fasting prior to the insulin tolerance test (Supplementary Fig. S5D).

\subsection{Dyslipidemia, hypercholesterolemia and increased glucose blood levels in mice fed with the Western diet}

Compared to controls, in unfasted animals, basal blood glucose levels were significantly increased in the WD group $(\mathrm{t}=4.484$, $\mathrm{P}=.0019$, Unpaired t-test, Fig. 3A). Basal (dark cycle) blood levels of total cholesterol, LDL, HDL and remnant cholesterol were increased in the mice fed the WD compared to control animals $(\mathrm{t}=10.65$, $\mathrm{P}<.0001, \mathrm{t}=7.656, \mathrm{P}<.0001 ; \mathrm{t}=11.01, \mathrm{P}<.0001$ and $\mathrm{t}=3.314$, $\mathrm{P}=.0109$, respectively, Unpaired t-test, Fig. 3B-E). There was no significant difference between the groups for the blood concentration of triglyceride $(\mathrm{t}=1.196, \mathrm{P}=.2630$, Unpaired t-test, Fig. 3F). 
A

Glucose

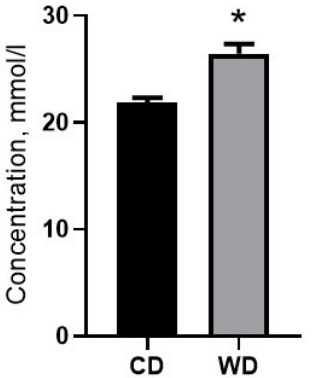

D

Low-density lipoprotein

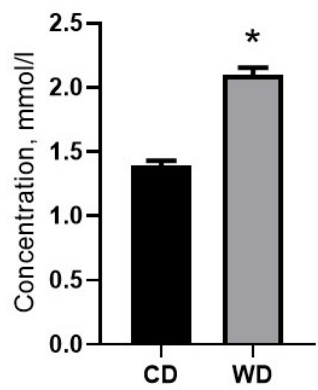

B

Total cholesterol

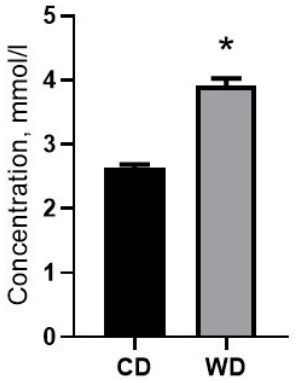

E

Remnant cholesterol

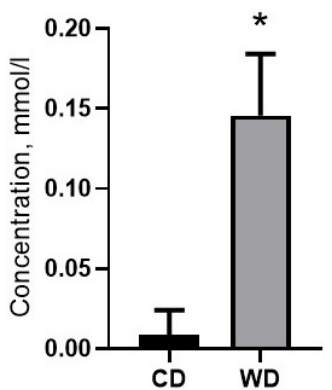

C

High-density

lipoprotein

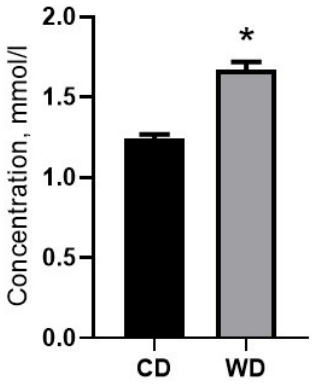

F

Triglycerides

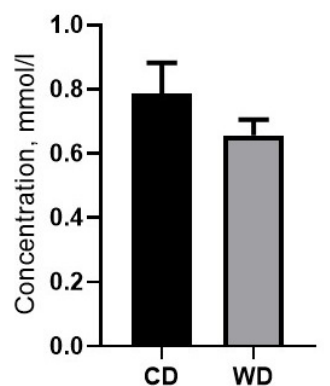

Fig. 3. The Western diet induces hypercholesterinemia and hyperglycemia. Blood concentrations of (A) non-fasting glucose, (B) total cholesterol, (C) low-density lipoproteins, (D) high-density lipoproteins and (E) remnant cholesterol were increased in mice fed a WD for 3 weeks compared to controls. (F) No difference was found in blood triglyceride concentration. CD Control diet, WD - Western diet ${ }^{*}-\mathrm{P}<.05$ compared to CD (Unpaired t-test). Data are presented as Mean \pm SEM. 


\subsection{A NASH-like syndrome induced by the Western diet}

The group receiving the WD displayed marked liver steatosis (Fig. 4A); the density of Oil Red O staining, which reveals liver triglycerides and lipids, was significantly increased in this group compared to controls $(t=26.57, \mathrm{P}<.0001$, Unpaired t-test, Fig. 4B). Significant increases in the blood concentration of ALT and AST, markers of hepatocellular injury, in WD-fed animals compared to control mice $(\mathrm{t}=2.517, \mathrm{P}=.0362$ and $\mathrm{t}=2.793, \mathrm{P}=.0213$, Unpaired t-test, Fig. 4C-D), which suggest the development of a NASH-like syndrome. No significant difference was found in blood urea concentration between the groups $(\mathrm{t}=1.254, \mathrm{P}=.2430$, Unpaired t-test, Fig. 4E). 




B

Liver

Oil Red 0

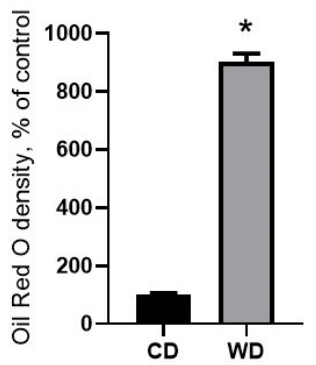

C

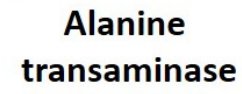

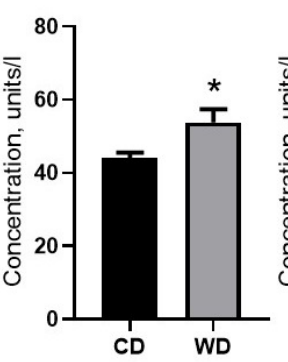

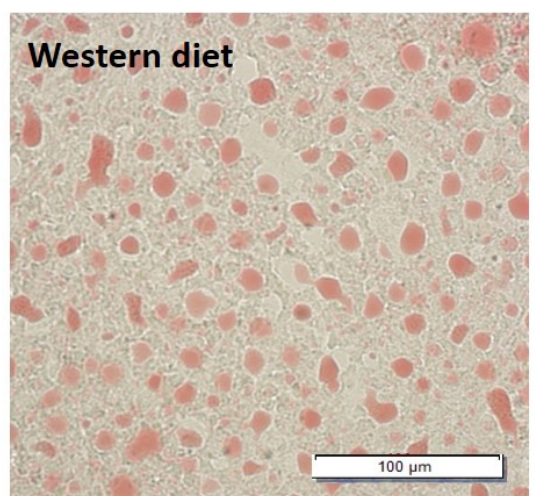

D

\section{Aspartate transaminase}
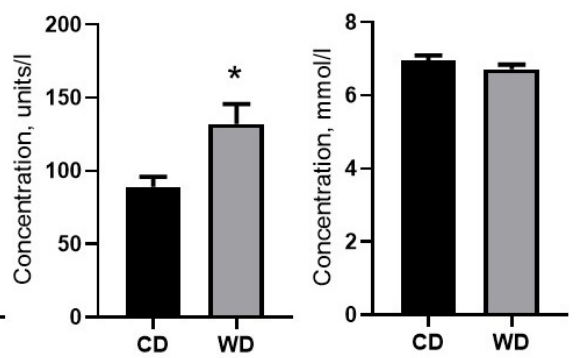

Fig. 4. NASH-like syndrome in mice fed with the Western diet. (A-B) In comparison to controls, mice fed a WD displayed marked liver steatosis: density of Oil Red O staining was increased in WD-fed mice compared controls. Compared to controls, there was a significant increase of blood concentrations of markers of hepatocellular injury (C) aspartate transaminase and (D) alanine transaminase in mice fed a WD. (E) No difference between the groups was found in urea blood concentration. CD - Control diet, WD - Western diet. * ${ }^{*} \mathrm{P}<.05$ compared to CD (Unpaired t-test). Data are presented as Mean \pm SEM. 
3.4. Decreased serotonin transporter expression, microglia activation and elevated marker of oxidative stress malondialdehyde in the prefrontal cortex of mice fed the Western diet

Levels of SERT expression were significantly decreased in the WD-fed group in the prefrontal cortex $(\mathrm{t}=1.471, \mathrm{P}<.0001$, Unpaired t-test, Fig. 5A), but not in the hippocampus $(\mathrm{t}=0.1133, \mathrm{P}=.9119$, Unpaired t-test, Fig. 5B) compared to controls. In mice fed the WD, the number of Iba1-positive cells was significantly increased in prefrontal cortex $(\mathrm{t}=2.670, \mathrm{P}=.0234$, Unpaired t-test $)$, but not in the hippocampus ( $\mathrm{t}=0.1089, \mathrm{P}=.9173$, Unpaired $\mathrm{t}$-test $)$ or in the motor cortex $(\mathrm{t}=0.3374, \mathrm{P}=.7414$, Unpaired t-test, Fig. 5C-F). The malondialdehyde concentration was significantly increased in animals fed the WD compared to mice fed control diet in the prefrontal cortex $(\mathrm{t}=2.515, \mathrm{P}=.0456$, Unpaired t-test, Fig. 5G), but level was unchanged in all the other sites in which it was measured (Fig. 5H-J). 

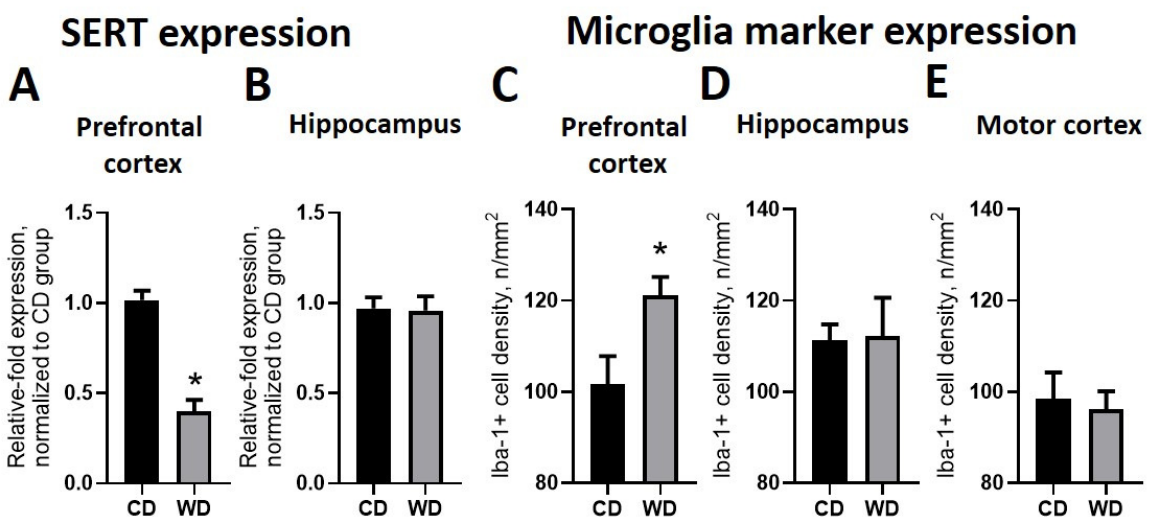

$\mathbf{F}$
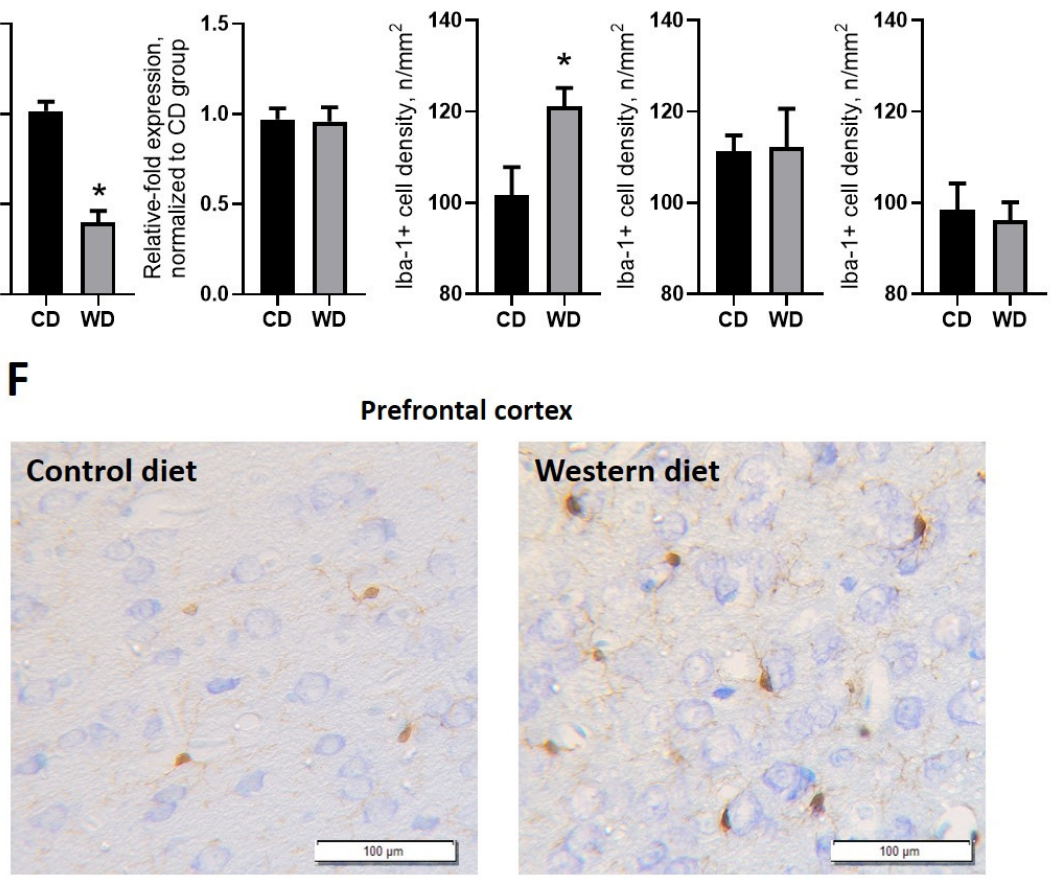

\section{Malondialdehyde concentration}

G

Prefrontal cortex

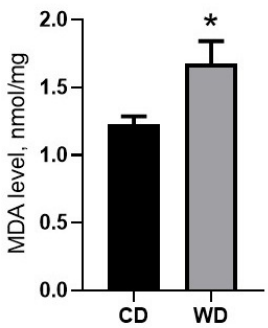

H

Hippocampus

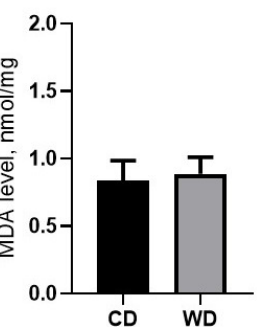

I

Hypothalamus

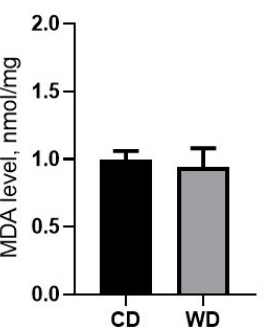

J

Dorsal raphe

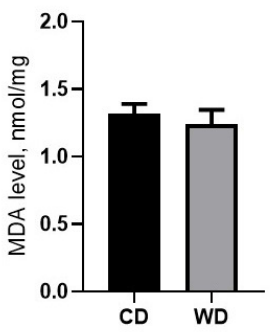

Fig. 5. The Western diet induces decreased serotonin transporter expression, signs of microglia activation and oxidative stress in prefrontal cortex. (A-B) Serotonin transporter expression in prefrontal cortex 
and in hippocampus. (C) The density of Iba1-positive cells in prefrontal cortex, (D) the hippocampus, and (E) motor cortex. (F) Representative staining of microglia in the prefrontal cortex of mice fed control or WD (magnification $\mathrm{x} 40$ ).

(G) Malondialdehyde concentration in the prefrontal cortex, $(\mathrm{H})$ the hippocampus,

(I) hypothalamus and (J) dorsal raphe. CD - Control diet, WD - Western diet. * $-\mathrm{P}<.05$ compared to CD (Unpaired t-test). Data are presented as Mean \pm SEM.

\subsection{The Western diet causes impaired motor performance and exploration, and behavioral despair}

In the last trial of the rotarod test, the latency to fall was significantly decreased in the WD-fed group compared to control mice $(\mathrm{U}=5, \mathrm{P}=.0346$, Mann-Whitney test, Fig. $\mathbf{6 A \& B})$. In the wire test, mice fed the WD demonstrated decreased latency to fall compared to controls ( $\mathrm{U}=3, \mathrm{P}=.0152$, Mann-Whitney test, Fig. 6C). Data from the two separate motor tests suggest that the WD-fed mice have impaired motor function.

In the pole test, which is a short-test requiring muscle strength and less coordination, there was no difference between the groups ( $\mathrm{U}=17, \mathrm{P}=.9416$, Mann-Whitney test, Fig. 6D). There was also no significant difference between the groups in gastrocnemius muscle weight $(\mathrm{t}=0.3773, \mathrm{P}=.7174$, Unpaired t-test, Fig. 6E) or the performance in 'Weights test' of muscle strength $(\mathrm{t}=0.2758$, $\mathrm{P}=.7890$, Unpaired t-test, Fig. 6F). 
A

\section{Acquisition of rotarod task}

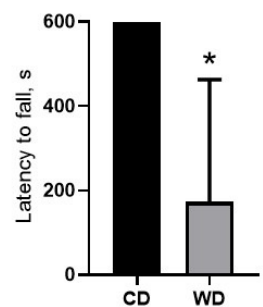

E

Muscle gastrocnemius

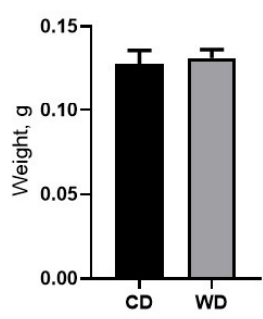

B

Dynamics of rotarod task

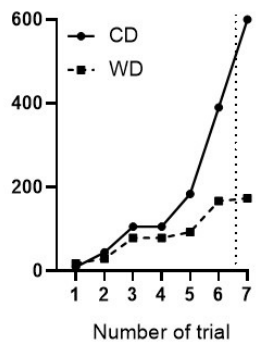

F

Weights test

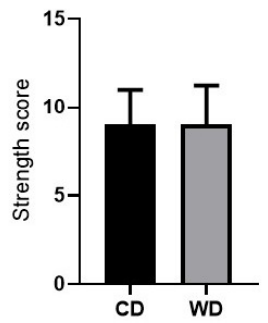

Forced swim test D1

I

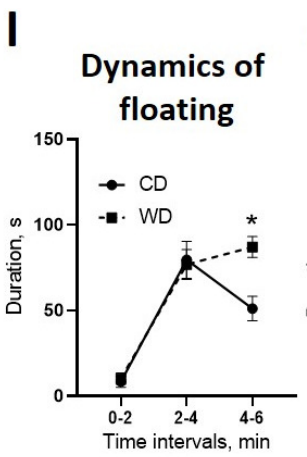

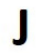

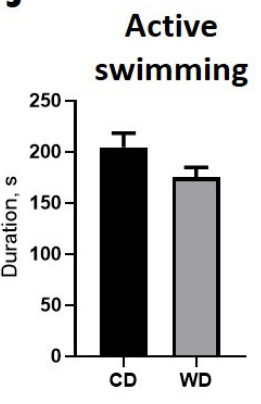

C

Wire test

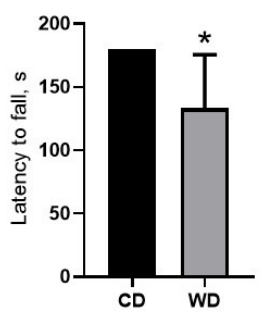

G

Object recognition

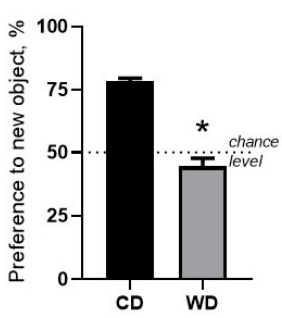

Forced swim test D2

K
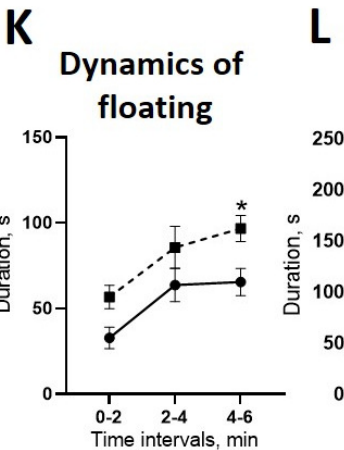

H

Object

exploration D1

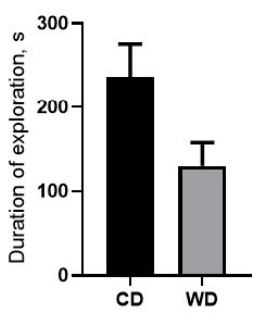

Pole test

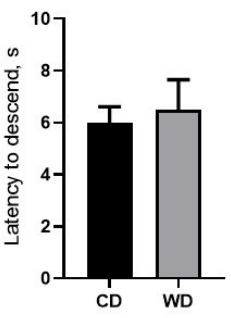

Active

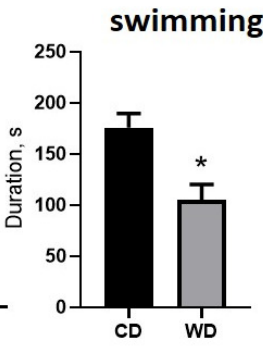

Fig. 6. Impaired motor performance, object recognition and behavioral despair in the forced swim test of mice fed with the Western diet. (A) The latency to fall in the last trial of the rotarod. Compared to controls, mice fed with the WD resulted in a significantly decreased. (B) The latency to fall during the repeated rotarod testing period. Control mice demonstrated an 
increased compared to the WD-fed animals. (C) Latency to fall in the wire test. Note the latency was significantly decreased in mice housed on a WD compared to control animals. (D) Latency to fall in the pole test. (E) Gastrocnemius muscle weight. (F) Muscle strength measured in the Weights test. (G) Object exploration/recognition test. The animals fed with the WD demonstrated significantly decreased preference to explore the novel object compared to control group. (H) Time spent with the objects on Day 1: there were no significant differences between groups. (I) Forced swim test. On Day 1 of the, the WD dietfed group showed significantly increased duration of floating during 4-6 min of the test and $(\mathrm{J})$ unaltered active swimming compared to mice fed control diet. $(\mathrm{K})$ Day 2 of the forced swim test results. The mice fed the WD displayed significantly increased duration of floating during 4-6 min of testing and (L) decreased duration of active swimming compared to controls. CD - Control diet, WD - Western diet. * $-\mathrm{P}<.05$ compared to CD (Unpaired t-test or Sidak's multiple comparisons test). Data are presented as Median with interquartile range (A,C,D), Median (B) or Mean \pm SEM (E-L).

There were no differences between the groups in the latency to fall from the inverted screen (Supplementary Fig. S6A). These tests argue against the possibility of muscle dystrophy in the WD-fed group as a contributor to the outcomes.

The preference to explore the novel object was decreased in mice housed on WD compared to mice housed on control diet $(\mathrm{t}=2.219$, $\mathrm{P}=.05$, Unpaired t-test, Fig. 6G, Supplementary Fig. S6B). These data suggest reduced novelty exploration in the WD-fed animals, which serves to demonstrate deficits in cognition in these mice. There was some evidence, from day 1 of the trial, that the animals fed the WD diet were less exploratory in the object training session, but, though close, the differences in time spent with the objects were not significant between groups $(\mathrm{t}=2.219, \mathrm{P}=.0609$, Unpaired t-test, Fig. $\mathbf{6 H})$. 
In the swim test, on day 1, two-way ANOVA for repeated measures revealed a significant interaction between test time interval and dietary group on floating duration $(\mathrm{F}=5.123, \mathrm{P}=.0140)$. WD-fed mice displayed a significantly increased duration of floating during 4$6 \mathrm{~min}$ of the test compared to controls $(\mathrm{t}=3.831, \mathrm{P}=.0075$, Sidak's multiple comparisons test), but not at other time intervals (Fig. 6I). The latency to float, total duration of floating, climbing behaviors and active swimming did not differ between the groups (Supplementary Fig. S7A-C, Fig. 6J).

In the swim test, on day 2, two-way ANOVA for repeated measures revealed significant effect of the $\operatorname{diet}(\mathrm{F}=8.550, \mathrm{P}=.0127)$ on the duration of floating. WD-fed mice displayed a significant increase in the duration of floating during $4-6 \mathrm{~min}$ of the test compared to controls $(\mathrm{t}=2.844, \mathrm{P}=.0438$, Sidak's multiple comparisons test, Fig. 6K), no significant differences were found for other intervals. Total duration of floating was increased in mice fed the WD (Supplementary Fig. S6E) compared to controls. The latency to float and duration of climbing behavior on day 2 did not differ between the groups (Supplementary Fig. S6D,F). The duration of active swimming on day 2 was increased in WD-fed mice compared to mice fed control $(\mathrm{t}=3.376, \mathrm{P}=.0056$, Unpaired t-test, Fig. 6L).

Separate studies have revealed unaltered open field horizontal locomotion of WD-exposed mice, under stress-free low light conditions 
(Supplementary Fig. S7). This observation argues against the possibility that the changes in the swim test, novelty exploration and memorizing, as well as altered floating behavior could be ascribed to general locomotor disturbances. Moreover, previous experiments revealed a lack of differences in the open field test activity in control and dietary challenged mice under conditions of stress-free subtle lighting [11]. In these studies, the dietary-challenged mice, which displayed unaltered speed and distance moved in the open field, had increased scores for floating in the swim test and immobilization in the tail suspension test, and spent a shorter time in the open arms of elevated O-maze and lit box of the dark/light box [11].

\section{Discussion}

The results presented here indicate that consumption of the WD changes gene expression and the cellularity of the prefrontal cortex, which is known to be involved in inhibitory behavioral control $[47,48]$. The three-week long period of WD feeding impaired motor coordination in the rotarod and wire test, in the absence of changes to muscle weight, strength, or body weight. Thus, the motor deficits were unlikely to be of neuro-muscular origin, and were most likely to be related to altered impulse control, and cognitive and emotional disturbances. The WD-fed mice exhibited down-regulated SERT expression, signs of microglial activation and oxidative stress, all in the 
prefrontal cortex, but not in the other structures of the brain that were investigated here. Impaired glucose and insulin tolerance, increased liver injury markers (AST and ALT), liver steatosis, and signs of dyslipidemia provided evidence for the development of metabolic and NASH syndromes in the WD-fed mice. However, unaltered blood urea levels suggest compensated liver function, ruling out a possibility of that the negative behavioral effects of the WD are simply due to encephalopathy-related mechanisms.

In our previous studies, mice fed the WD for 21-days were found to display behavioral abnormalities that were reminiscent of certain key ADHD-like symptoms: impulsivity [11], hyperactivity [13], autistic-like traits in social interaction tests [13], and emotional disturbances in classic tests for anxiety and behavioral despair [12]. These data, together with the present findings, show that the mice fed the WD for 3 weeks can generate motor deficits and signs of compromised function of the prefrontal cortex further supports the argument that the mouse behaviors resemble those of the ADHD and ASD. It has been extensively reported that ADHD and ASD are characterized in humans by decreased activation in the medial prefrontal cortex $[25,49]$, where suppressed integrative functionality is thought to underpin many of the behaviors. In our experiments, a number of the changes, such as a reduction in the SERT expression, the appearance of oxidative stress markers and microglial activation, 
occurred in in the prefrontal cortex alone, but the explanation for why this part of the brain should be sensitive to the WD remains unclear. An increase in the susceptibility of prefrontal cortex to the WD was also highlighted in a previous study where we found increased protein expression of Toll-like receptor 4 (TLR4), a major regulator of proinflammatory response and microglial activation, in the prefrontal cortex but not elsewhere [11]. It is a part of the brain region that is most sensitive to the effects of stress exposure. Even mild acute uncontrollable stress can cause a rapid and dramatic loss of prefrontal cognitive abilities, and more prolonged stress exposure causes architectural changes in prefrontal dendrites. Others have shown that there is an interaction between stress and diet on the structure of the prefrontal cortex [50], but the relationship is complex and it remains unclear why regional differences exist.

Decreased SERT expression, observed in the brain of the WD-fed animals, has previously been reported to be associated with depressive symptoms [51], decreased sociability, impaired glucose tolerance [52,53], neuroinflammation [54] and the mechanisms underpinning ASD $[55,56]$ and ADHD [32,57]. Thus, a downregulation of SERT in the prefrontal cortex might also contribute to the deficits reported in the present study. Other animal studies have also reported changes in the expression of SERT mRNA/protein in peripheral organs in rodents fed with the WD [29]. Human studies describe reduced SERT expression 
in a brain and periphery of subjects with metabolic syndrome and obesity $[30,31]$ and suggest a role of SERT polymorphism, regulating its activity, in controlling body weight $[58,59]$, development of diabetes type II [60] and eating behavior [61].

The present literature also reports a negative relationship between SERT expression and neuroinflammation [54,62], which appears to be a key feature in mice fed a WD. Remarkably, the present study revealed increases of Iba1-positive cells and the concentration of MDA, a marker of oxidative stress, which is suggestive of proinflammatory processes [63]. The increase in Iba1-positive cells in the prefrontal cortex of WD-fed animals is in keeping with previous observations in rat and mouse models of obesity, after prolonged feeding, that have reported increases in Iba1-positive cell in the hippocampus [64,65], prefrontal cortex [66] and hypothalamus [67,68]. The increase in the oxidative stress marker (MDA) is also in keeping with the evidence for similar changes in a periphery in obese humans [69-71]. Juvenile mice fed a WD for 4 weeks displayed increased protein oxidation and lipid peroxidation in the frontal cortex [72]. Most animal studies that have described elevated brain oxidative stress markers in models of prolonged intake of the WD are also associated with obesity [73-76]. As a consequence, it has been hard to determine whether the downstream obesity or the WD per se is responsible for the molecular and cellular changes. Here, our results demonstrate that the 
inflammatory changes develop in non-obese rodents after a relatively short period of WD feeding.

Previous studies employing longer feeding periods than employed here have demonstrated that motor deficits can be induced by the WD. These motor deficits include impaired performance in the balance beam test [9], rotarod, pole, stepping and hind-limb clasping tests [22]. The present study is one of few observations that reports motor deficits to be associated with a short period of housing on the WD, and excludes potential artifacts in behavioral analysis owing to obesity $[9,22,77]$. Here we found that the WD impaired mouse performance in the wire test and rotarod, but not in the pole test. A lack of deficits in the muscle weight and strength in our experiments suggest that deficient coordination of mice housed on WD is unlikely to be related to a dysfunction of primary elements of the motor system, but are likely to be owing to cognitive and emotional changes under these conditions $[9,21,78,79]$.

The inflammatory changes in the prefrontal cortex are likely to have been driven, as least in part by the molecular changes observed in the liver. It is becoming clear that perturbation of the gut-liver-brain axis can result in altered behavior. The animals displayed elevated serum AST and ALT, classic biomarkers of liver injury that is known to accompany obesity in humans [80] and rodents [81] and hepatitis in mice that is not associated with being over-weight $[82,83]$. There was 
also a marked liver steatosis, which provides the evidence for the development of a NASH-like syndrome, which is characterized by proinflammatory changes of that is known to induce depressive-like and anxiety-like changes $[43,46]$. Increased blood levels of total cholesterol, LDL, HDL and remnant cholesterol in dietary challenged animals provide further evidence the development of systemic proinflammatory changes in our study. For example, high remnant cholesterol is associated with chronic inflammation, and is a strong predictor of myocardial infarction in people with normal weight $[84,85]$. Finally, insulin resistance and impaired glucose tolerance in mice housed on the WD suggest possible dysfunction in the central insulin receptor-mediated processes that play crucial in emotional regulation [86].

\section{Conclusion}

Taken together, these data suggest the importance of a WD in the development of neuropsychiatric symptoms, and, in particular, of ASD-like and ADHD-like behaviors in adulthood and highlight an early role for the prefrontal cortex in the development of these behaviors. Abnormal intake of highly caloric food and metabolic abnormalities are often observed in the ADHD patients where they might have additive or synergistic effects on symptoms [87-89]. Thus our results suggest that a WD is likely to impact on the pathogenesis of neuro- 
developmentally acquired syndromes such as ADHD and ASD and that further investigation in human studies is warranted [90].

\section{Declaration of competing interest}

On behalf of all authors, the corresponding author states that there is no conflict of interest.

\section{Acknowledgments}

The authors' work reported here was supported by the European Community (the Horizon 2020 Research and Innovation Programme under Grant No. 728018 Eat2beNICE), the "5-100" Russian Academic Excellence Project (to KPL and TS) and the Russian Science Foundation (RSF Grant No. 16-16-10073 to EK and LF). We appreciate valuable technical help of Anna Gorlova, Anastasiya Kibitkina, Galina Tolmacheva, Diana Babayevskaya and Igor Shafarevich with this project. LB is Research Director of the F.R.S.FNRS (Belgium).

\section{References}

[1] L. Cordain, S.B. Eaton, A. Sebastian, N. Mann, S. Lindeberg, B.A. Watkins, J.H. O'Keefe, J. Brand-Miller, Origins and evolution of the Western diet: health implications for the 21st century, Am. J. Clin. Nutr. 81 (2005) 341-354. doi:10.1093/ajcn.81.2.341.

[2] K. Okręglicka, Health effects of changes in the structure of dietary macronutrients intake in western societies, Rocz. Panstw. Zakl. Hig. 66 (2015) 97-105. http://www.ncbi.nlm.nih.gov/pubmed/26024397. 
[3] F.N. Jacka, J.A. Pasco, A. Mykletun, L.J. Williams, A.M. Hodge, S.L. O'Reilly, G.C. Nicholson, M.A. Kotowicz, M. Berk, Association of Western and Traditional Diets With Depression and Anxiety in Women, Am. J. Psychiatry. 167 (2010) 305-311. doi:10.1176/appi.ajp.2009.09060881.

[4] M. Khosravi, G. Sotoudeh, R. Majdzadeh, S. Nejati, S. Darabi, F. Raisi, A. Esmaillzadeh, M. Sorayani, Healthy and Unhealthy Dietary Patterns Are Related to Depression: A Case-Control Study, Psychiatry Investig. 12 (2015) 434-42. doi:10.4306/pi.2015.12.4.434.

[5] W.H. Oddy, K.L. Allen, G.S.A. Trapp, G.L. Ambrosini, L.J. Black, R.-C. Huang, P. Rzehak, K.C. Runions, F. Pan, L.J. Beilin, T.A. Mori, Dietary patterns, body mass index and inflammation: Pathways to depression and mental health problems in adolescents, Brain. Behav. Immun. 69 (2018) 428-439. doi:10.1016/j.bbi.2018.01.002.

[6] N. Connolly, J. Anixt, P. Manning, D. Ping-I Lin, K.A. Marsolo, K. Bowers, Maternal metabolic risk factors for autism spectrum disorder-An analysis of electronic medical records and linked birth data, Autism Res. 9 (2016) 829-837. doi:10.1002/aur.1586.

[7] A. Kawicka, B. Regulska-Ilow, How nutritional status, diet and dietary supplements can affect autism. A review, Rocz. Panstw. Zakl. Hig. 64 (2013) 1-12. http://www.ncbi.nlm.nih.gov/pubmed/23789306.

[8] J.G. Millichap, M.M. Yee, The Diet Factor in AttentionDeficit/Hyperactivity Disorder, Pediatrics. $129 \quad$ (2012) 330-337. doi:10.1542/peds.2011-2199.

[9] K. Takase, Y. Tsuneoka, S. Oda, M. Kuroda, H. Funato, High-fat diet feeding alters olfactory-, social-, and reward-related behaviors of mice independent of obesity, Obesity. 24 (2016) 886-894. doi:10.1002/oby.21441.

[10] E. Vagena, J.K. Ryu, B. Baeza-Raja, N.M. Walsh, C. Syme, J.P. Day, M.D. Houslay, G.S. Baillie, A high-fat diet promotes depression-like behavior in mice by suppressing hypothalamic PKA signaling, Transl. Psychiatry. 9 (2019) 141. doi:10.1038/s41398-019-0470-1.

[11] T. Strekalova, M. Evans, J. Costa-Nunes, S. Bachurin, N. Yeritsyan, Y. Couch, H.M.W. Steinbusch, S.E. Köhler, K.-P. Lesch, D.C. Anthony, Tlr4 upregulation in the brain accompanies depression- and anxiety-like behaviors induced by a high-cholesterol diet, Brain. Behav. Immun. 48 (2015). doi:10.1016/j.bbi.2015.02.015.

[12] T. Strekalova, J.P. Costa-Nunes, E. Veniaminova, A. Kubatiev, K.P. Lesch, V.P. Chekhonin, M.C. Evans, H.W.M. Steinbusch, Insulin receptor sensitizer, dicholine succinate, prevents both Toll-like receptor 4 (TLR4) 
upregulation and affective changes induced by a high-cholesterol diet in mice, J. Affect. Disord. 196 (2016) 109-116. doi:10.1016/j.jad.2016.02.045.

[13] E. Veniaminova, R. Cespuglio, C.W. Cheung, A. Umriukhin, N. Markova, E. Shevtsova, K.-P. Lesch, D.C. Anthony, T. Strekalova, AutismLike Behaviours and Memory Deficits Result from a Western Diet in Mice, Neural Plast. 2017 (2017) 1-14. doi:10.1155/2017/9498247.

[14] E. Veniaminova, R. Cespuglio, N. Markova, N. Mortimer, C. Wai Cheung, H.W. Steinbusch, K.-P. Lesch, T. Strekalova, Behavioral features of mice fed with a cholesterol-enriched diet:Deficient novelty exploration and unaltered aggressive behavior, Transl. Neurosci. Clin. 2 (2016) 87. doi:10.18679/CN11-6030/R.2016.014.

[15] H. Faras, N. Al Ateeqi, L. Tidmarsh, Autism spectrum disorders, Ann. Saudi Med. 30 (2010) 295-300. doi:10.4103/0256-4947.65261.

[16] L.E. Knouse, I. Zvorsky, S.A. Safren, Depression in Adults with Attention-Deficit/Hyperactivity Disorder (ADHD): The Mediating Role of Cognitive-Behavioral Factors, Cognit. Ther. Res. 37 (2013) 1220-1232. doi:10.1007/s10608-013-9569-5.

[17] T.E. Wilens, T.J. Spencer, Understanding attentiondeficit/hyperactivity disorder from childhood to adulthood, Postgrad. Med. 122 (2010) 97-109. doi:10.3810/pgm.2010.09.2206.

[18] N. Cheng, J.M. Rho, S.A. Masino, Metabolic Dysfunction Underlying Autism Spectrum Disorder and Potential Treatment Approaches, Front. Mol. Neurosci. 10 (2017). doi:10.3389/fnmol.2017.00034.

[19] S. Hoirisch-Clapauch, A.E. Nardi, Autism spectrum disorders: let's talk about glucose?, Transl. Psychiatry. 9 (2019) 51. doi:10.1038/s41398-0190370-4.

[20] Z. Landau, O. Pinhas-Hamiel, Attention Deficit/Hyperactivity, the Metabolic Syndrome, and Type 2 Diabetes, Curr. Diab. Rep. 19 (2019) 46. doi:10.1007/s11892-019-1174-x.

[21] D. Gaul, A. Mat, D. O'Shea, J. Issartel, Impaired Visual Motor Coordination in Obese Adults, J. Obes. 2016 (2016) 1-8. doi:10.1155/2016/6178575.

[22] A. Stojakovic, C.A. Mastronardi, J. Licinio, M.-L. Wong, Long-term consumption of high-fat diet impairs motor coordination without affecting the general motor activity, J. Transl. Sci. 5 (2018) 1-10. doi:10.15761/JTS.1000295.

[23] E. Dayan, L.G. Cohen, Neuroplasticity Subserving Motor Skill Learning, Neuron. 72 (2011) 443-454. doi:10.1016/j.neuron.2011.10.008. 
[24] A. Yasumura, M. Omori, A. Fukuda, J. Takahashi, Y. Yasumura, E. Nakagawa, T. Koike, Y. Yamashita, T. Miyajima, T. Koeda, M. Aihara, H. Tachimori, M. Inagaki, Applied Machine Learning Method to Predict Children With ADHD Using Prefrontal Cortex Activity: A Multicenter Study in Japan, J. Atten. Disord. (2017) 108705471774063. doi: $10.1177 / 1087054717740632$.

[25] D. Ben Shalom, The Medial Prefrontal Cortex and Integration in Autism, Neurosci. 15 (2009) 589-598. doi:10.1177/1073858409336371.

[26] B. Lizarbe, A.F. Soares, S. Larsson, J.M.N. Duarte, Neurochemical Modifications in the Hippocampus, Cortex and Hypothalamus of Mice Exposed to Long-Term High-Fat Diet, Front. Neurosci. 12 (2019). doi:10.3389/fnins.2018.00985.

[27] T.M. Comhair, S.C. Garcia Caraballo, C.H. Dejong, W.H. Lamers, S.E. Koehler, Dietary cholesterol, female gender and n-3 fatty acid deficiency are more important factors in the development of non-alcoholic fatty liver disease than the saturation index of the fat, Nutr. Metab. (Lond). 8 (2011) 4. doi:10.1186/1743-7075-8-4.

[28] S. Tromans, V. Chester, R. Kiani, R. Alexander, T. Brugha, The Prevalence of Autism Spectrum Disorders in Adult Psychiatric Inpatients: A Systematic Review, Clin. Pract. Epidemiol. Ment. Heal. 14 (2018) 177-187. doi:10.2174/1745017901814010177.

[29] R.L. Bertrand, S. Senadheera, I. Markus, L. Liu, L. Howitt, H. Chen, T. V. Murphy, S.L. Sandow, P.P. Bertrand, A Western Diet Increases Serotonin Availability in Rat Small Intestine, Endocrinology. 152 (2011) 3647. doi:10.1210/en.2010-0377.

[30] G. Giannaccini, L. Betti, L. Palego, A. Marsili, F. Santini, C. Pelosini, L. Fabbrini, L. Schmid, L. Giusti, M. Maffei, M. Lanza, M. Cristofaro, S. Baroni, M. Mauri, P. Vitti, P. Fierabracci, A. Lucacchini, The expression of platelet serotonin transporter (SERT) in human obesity, BMC Neurosci. 14 (2013) 128. doi:10.1186/1471-2202-14-128.

[31] S.B. Nam, K. Kim, B.S. Kim, H.-J. Im, S.H. Lee, S.-J. Kim, I.J. Kim, K. Pak, The Effect of Obesity on the Availabilities of Dopamine and Serotonin Transporters, Sci. Rep. 8 (2018) 4924. doi:10.1038/s41598-01822814-8.

[32] T. Vanicek, A. Kutzelnigg, C. Philippe, H.L. Sigurdardottir, G.M. James, A. Hahn, G.S. Kranz, A. Höflich, A. Kautzky, T. Traub-Weidinger, M. Hacker, W. Wadsak, M. Mitterhauser, S. Kasper, R. Lanzenberger, Altered interregional molecular associations of the serotonin transporter in 
attention deficit/hyperactivity disorder assessed with PET, Hum. Brain Mapp. 38 (2017) 792-802. doi:10.1002/hbm.23418.

[33] C. Chang, S.S.-F. Gau, W.-S. Huang, C.-Y. Shiue, C.-B. Yeh, Abnormal serotonin transporter availability in the brains of adults with conduct disorder, J. Formos. Med. Assoc. 116 (2017) 469-475. doi:10.1016/j.jfma.2016.07.012.

[34] J. de Munter, I. Schafarevich, A. Liundup, D. Pavlov, E. Wolters, A. Gorlova, E. Veniaminova, A. Umriukhin, A. Kalueff, A. Svistunov, B.W. Kramer, K.-P. Lesch, T. Strekalova, C.N. and therapeutics in Press, NeuroCell therapy improves motor outcomes and suppresses inflammation during experimental syndrome of amyotrophic lateral sclerosis in mice, CNS Neurosci. Ther. (2019). doi:10.1111/cns.13280.

[35] R.M.J. Deacon, Measuring the Strength of Mice, J. Vis. Exp. (2013). doi:10.3791/2610.

[36] W. Kondziella, A new method for the measurement of muscle relaxation in white mice, Arch. Int. Pharmacodyn. Ther. 152 (1964) 277-84. http://www.ncbi.nlm.nih.gov/pubmed/14265648.

[37] T. Strekalova, D.C. Anthony, O. Dolgov, K. Anokhin, A. Kubatiev, H.M.W. Steinbusch, C. Schroeter, The differential effects of chronic imipramine or citalopram administration on physiological and behavioral outcomes in naïve mice, Behav. Brain Res. 245 (2013) 101-106. doi:10.1016/j.bbr.2013.02.015.

[38] T. Strekalova, R. Spanagel, D. Bartsch, F.A. Henn, P. Gass, StressInduced Anhedonia in Mice is Associated with Deficits in Forced Swimming and Exploration, Neuropsychopharmacology. $29 \quad$ (2004) 2007. https://doi.org/10.1038/sj.npp.1300532.

[39] E. Malatynska, H.W.M. Steinbusch, O. Redkozubova, A. Bolkunov, A. Kubatiev, N.B. Yeritsyan, J. Vignisse, S. Bachurin, T. Strekalova, Anhedonic-like traits and lack of affective deficits in 18-month-old C57BL/6 mice: Implications for modeling elderly depression, Exp. Gerontol. 47 (2012) 552-564. doi:10.1016/j.exger.2012.04.010.

[40] B.H. Cline, J.P. Costa-Nunes, R. Cespuglio, N. Markova, A.I. Santos, Y.V. Bukhman, A. Kubatiev, H.W.M. Steinbusch, K.-P. Lesch, T. Strekalova, Dicholine succinate, the neuronal insulin sensitizer, normalizes behavior, REM sleep, hippocampal pGSK3 beta and mRNAs of NMDA receptor subunits in mouse models of depression, Front. Behav. Neurosci. 9 (2015). doi:10.3389/fnbeh.2015.00037. 
[41] J.P. Costa-Nunes, B.H. Cline, M. Araújo-Correia, A. Valença, N. Markova, O. Dolgov, A. Kubatiev, N. Yeritsyan, H.W.M. Steinbusch, T. Strekalova, Animal Models of Depression and Drug Delivery with Food as an Effective Dosing Method: Evidences from Studies with Celecoxib and Dicholine Succinate, Biomed Res. Int. 2015 (2015) 596126. doi: $10.1155 / 2015 / 596126$.

[42] J.E. Ayala, V.T. Samuel, G.J. Morton, S. Obici, C.M. Croniger, G.I. Shulman, D.H. Wasserman, O.P. McGuinness, NIH Mouse Metabolic Phenotyping Center Consortium, Standard operating procedures for describing and performing metabolic tests of glucose homeostasis in mice, Dis. Model. Mech. 3 (2010) 525-34. doi:10.1242/dmm.006239.

[43] Y. Couch, A. Trofimov, N. Markova, V. Nikolenko, H.W. Steinbusch, V. Chekhonin, C. Schroeter, K.-P. Lesch, D.C. Anthony, T. Strekalova, Lowdose lipopolysaccharide (LPS) inhibits aggressive and augments depressive behaviours in a chronic mild stress model in mice, J. Neuroinflammation. 13 (2016) 108. doi:10.1186/s12974-016-0572-0.

[44] D. Pavlov, L. Bettendorff, A. Gorlova, A. Olkhovik, A. V Kalueff, E.D. Ponomarev, A. Inozemtsev, V. Chekhonin, K.-P. Lesch, D.C. Anthony, T. Strekalova, Neuroinflammation and aberrant hippocampal plasticity in a mouse model of emotional stress evoked by exposure to ultrasound of alternating frequencies., Prog. Neuropsychopharmacol. Biol. Psychiatry. 90 (2019) 104-116. doi:10.1016/j.pnpbp.2018.11.014.

[45] A. Mehlem, C.E. Hagberg, L. Muhl, U. Eriksson, A. Falkevall, Imaging of neutral lipids by oil red $\mathrm{O}$ for analyzing the metabolic status in health and disease, Nat. Protoc. 8 (2013) 1149-1154. doi:10.1038/nprot.2013.055.

[46] Y. Couch, D.C. Anthony, O. Dolgov, A. Revischin, B. Festoff, A.I. Santos, H.W. Steinbusch, T. Strekalova, Microglial activation, increased TNF and SERT expression in the prefrontal cortex define stress-altered behaviour in mice susceptible to anhedonia, Brain. Behav. Immun. 29 (2013) 136-146. doi:10.1016/j.bbi.2012.12.017.

[47] S. Heinitz, M. Reinhardt, P. Piaggi, C.M. Weise, E. Diaz, E.J. Stinson, C. Venti, S.B. Votruba, E.M. Wassermann, M. Alonso-Alonso, J. Krakoff, M.E. Gluck, Neuromodulation directed at the prefrontal cortex of subjects with obesity reduces snack food intake and hunger in a randomized trial, Am. J. Clin. Nutr. 106 (2017) 1347-1357. doi:10.3945/ajcn.117.158089. [48] D.S.N. Le, N. Pannacciulli, K. Chen, A. Del Parigi, A.D. Salbe, E.M. Reiman, J. Krakoff, Less activation of the left dorsolateral prefrontal cortex 
in response to a meal: a feature of obesity, Am. J. Clin. Nutr. 84 (2006) 725731. doi:10.1093/ajcn/84.4.725.

[49] S. Kim, D. Lee, Prefrontal Cortex and Impulsive Decision Making, Biol. Psychiatry. 69 (2011) 1140-1146. doi:10.1016/j.biopsych.2010.07.005.

[50] M. Rincel, A.L. Lépinay, Y. Janthakhin, G. Soudain, S. Yvon, S. Da Silva, C. Joffre, A. Aubert, A. Séré, S. Layé, V. Theodorou, G. Ferreira, M. Darnaudéry, Maternal high-fat diet and early life stress differentially modulate spine density and dendritic morphology in the medial prefrontal cortex of juvenile and adult rats, Brain Struct. Funct. 223 (2018) 883-895. doi:10.1007/s00429-017-1526-8.

[51] J.R. Homberg, K.-P. Lesch, Looking on the bright side of serotonin transporter gene variation., Biol. Psychiatry. 69 (2011) 513-9. doi:10.1016/j.biopsych.2010.09.024.

[52] J.R. Homberg, S.E. la Fleur, E. Cuppen, Serotonin transporter deficiency increases abdominal fat in female, but not male rats, Obesity. 18 (2010) 137-145. doi:10.1038/oby.2009.139.

[53] D.L. Murphy, K.-P. Lesch, Targeting the murine serotonin transporter: insights into human neurobiology, Nat. Rev. Neurosci. 9 (2008) 85-96. doi:10.1038/nrn2284.

[54] A. Metaxas, R. Vaitheeswaran, K.T. Jensen, C. Thygesen, L. Ilkjaer, S. Darvesh, B. Finsen, Reduced serotonin transporter levels and inflammation in the midbrain raphe of 12 month old APPswe/PSEN1dE9 mice, Curr. Alzheimer Res. 15 (2018) 420-428. doi:10.2174/1567205014666171004113537. [55] R. Rose'Meyer, A review of the serotonin transporter and prenatal cortisol in the development of autism spectrum disorders, Mol. Autism. 4 (2013) 37. doi:10.1186/2040-2392-4-37.

[56] C.H. Lew, K.L. Hanson, K.M. Groeniger, D. Greiner, D. Cuevas, B. Hrvoj-Mihic, C.M. Schumann, K. Semendeferi, Serotonergic innervation of the human amygdala and evolutionary implications, Am. J. Phys. Anthropol. (2019). doi:10.1002/ajpa.23896.

[57] Z. Chang, P.D. Quinn, K. Hur, R.D. Gibbons, A. Sjölander, H. Larsson, B.M. D'Onofrio, Association Between Medication Use for AttentionDeficit/Hyperactivity Disorder and Risk of Motor Vehicle Crashes, JAMA Psychiatry. 74 (2017) 597. doi:10.1001/jamapsychiatry.2017.0659.

[58] B.F. Fuemmeler, T.D. Agurs-Collins, F.J. Mcclernon, S.H. Kollins, M.E. Kail, A.W. Bergen, A.E. Ashley-Koch, Genes Implicated in Serotonergic and Dopaminergic Functioning Predict BMI Categories, Obesity. 16 (2008) 348-355. doi:10.1038/oby.2007.65. 
[59] S. Sookoian, C. Gemma, S.I. García, T. Fernández Gianotti, G. Dieuzeide, A. Roussos, M. Tonietti, L. Trifone, D. Kanevsky, C.D. González, C.J. Pirola, Short Allele of Serotonin Transporter Gene Promoter Is a Risk Factor for Obesity in Adolescents*, Obesity. 15 (2007) 271-276. doi:10.1038/oby.2007.519.

[60] M. Iordanidou, A. Tavridou, I. Petridis, K.I. Arvanitidis, D. Christakidis, V. Vargemezis, V.G. Manolopoulos, The serotonin transporter promoter polymorphism (5-HTTLPR) is associated with type 2 diabetes, Clin. Chim. Acta. 411 (2010) 167-171. doi:10.1016/j.cca.2009.10.022.

[61] P. Monteleone, P. Santonastaso, M. Mauri, L. Bellodi, S. Erzegovesi, A. Fuschino, A. Favaro, A. Rotondo, E. Castaldo, M. Maj, Investigation of the Serotonin Transporter Regulatory Region Polymorphism in Bulimia Nervosa: Relationships to Harm Avoidance, Nutritional Parameters, and Psychiatric Comorbidity, Psychosom. Med. 68 (2006) 99-103. doi:10.1097/01.psy.0000195746.52074.63.

[62] J. Haase, E. Brown, Integrating the monoamine, neurotrophin and cytokine hypotheses of depression - A central role for the serotonin transporter?, Pharmacol. Ther. 147 (2015) 1-11. doi:10.1016/j.pharmthera.2014.10.002.

[63] J. Lugrin, N. Rosenblatt-Velin, R. Parapanov, L. Liaudet, The role of oxidative stress during inflammatory processes, Biol. Chem. 395 (2014) 203230. doi:10.1515/hsz-2013-0241.

[64] T. Chunchai, W. Thunapong, S. Yasom, K. Wanchai, S. Eaimworawuthikul, G. Metzler, A. Lungkaphin, A. Pongchaidecha, S. Sirilun, C. Chaiyasut, W. Pratchayasakul, P. Thiennimitr, N. Chattipakorn, S.C. Chattipakorn, Decreased microglial activation through gut-brain axis by prebiotics, probiotics, or synbiotics effectively restored cognitive function in obese-insulin resistant rats, J. Neuroinflammation. $15 \quad$ (2018) 11. doi:10.1186/s12974-018-1055-2.

[65] E.C. Cope, E.A. LaMarca, P.K. Monari, L.B. Olson, S. Martinez, A.D. Zych, N.J. Katchur, E. Gould, Microglia Play an Active Role in ObesityAssociated Cognitive Decline, J. Neurosci. 38 (2018) 8889-8904. doi:10.1523/JNEUROSCI.0789-18.2018.

[66] M.E. Bocarsly, M. Fasolino, G.A. Kane, E.A. LaMarca, G.W. Kirschen, I.N. Karatsoreos, B.S. McEwen, E. Gould, Obesity diminishes synaptic markers, alters microglial morphology, and impairs cognitive function, Proc. Natl. Acad. Sci. (2015) 201511593. doi:10.1073/pnas.1511593112. 
[67] Y. Gao, N. Ottaway, S.C. Schriever, B. Legutko, C. García-Cáceres, E. de la Fuente, C. Mergen, S. Bour, J.P. Thaler, R.J. Seeley, J. Filosa, J.E. Stern, D. Perez-Tilve, M.W. Schwartz, M.H. Tschöp, C.-X. Yi, Hormones and diet, but not body weight, control hypothalamic microglial activity, Glia. 62 (2014) 17-25. doi:10.1002/glia.22580.

[68] J.P. Thaler, C.-X. Yi, E.A. Schur, S.J. Guyenet, B.H. Hwang, M.O. Dietrich, X. Zhao, D.A. Sarruf, V. Izgur, K.R. Maravilla, H.T. Nguyen, J.D. Fischer, M.E. Matsen, B.E. Wisse, G.J. Morton, T.L. Horvath, D.G. Baskin, M.H. Tschöp, M.W. Schwartz, Obesity is associated with hypothalamic injury in rodents and humans, J. Clin. Invest. 122 (2012) 153-162. doi:10.1172/JCI59660.

[69] H.K. Vincent, A.G. Taylor, Biomarkers and potential mechanisms of obesity-induced oxidant stress in humans, Int. J. Obes. 30 (2006) 400-418. doi:10.1038/sj.ijo.0803177.

[70] N. Karaouzene, H. Merzouk, M. Aribi, S.A. Merzouk, A. Yahia Berrouiguet, C. Tessier, M. Narce, Effects of the association of aging and obesity on lipids, lipoproteins and oxidative stress biomarkers: A comparison of older with young men, Nutr. Metab. Cardiovasc. Dis. 21 (2011) 792-799. doi:10.1016/j.numecd.2010.02.007.

[71] F.J. Tinahones, M. Murri-Pierri, L. Garrido-Sánchez, J.M. GarcíaAlmeida, S. García-Serrano, J. García-Arnés, E. García-Fuentes, Oxidative Stress in Severely Obese Persons Is Greater in Those With Insulin Resistance, Obesity. 17 (2009) 240-246. doi:10.1038/oby.2008.536.

[72] C.M. Studzinski, F. Li, A.J. Bruce-Keller, S.O. Fernandez-Kim, L. Zhang, A.M. Weidner, W.R. Markesbery, M.P. Murphy, J.N. Keller, Effects of short-term Western diet on cerebral oxidative stress and diabetes related factors in APP × PS1 knock-in mice, J. Neurochem. 108 (2009) 860-866. doi:10.1111/j.1471-4159.2008.05798.x.

[73] K.A. Amin, H.H. Kamel, M.A. Abd Eltawab, The relation of high fat diet, metabolic disturbances and brain oxidative dysfunction: modulation by hydroxy citric acid., Lipids Health Dis. 10 (2011) 74. doi:10.1186/1476-511X10-74.

[74] C.C. de Aquino, R.A. Leitão, L.A. Oliveira Alves, V. Coelho-Santos, R.L. Guerrant, C.F. Ribeiro, J.O. Malva, A.P. Silva, R.B. Oriá, Effect of Hypoproteic and High-Fat Diets on Hippocampal Blood-Brain Barrier Permeability and Oxidative Stress, Front. Nutr. 5 (2019). doi:10.3389/fnut.2018.00131. 
[75] L.R. Freeman, V. Haley-Zitlin, D.S. Rosenberger, A.-C. Granholm, Damaging effects of a high-fat diet to the brain and cognition: a review of proposed mechanisms., Nutr. Neurosci. 17 (2014) 241-51. doi:10.1179/1476830513Y.0000000092.

[76] W. Ma, L. Yuan, H. Yu, Y. Xi, R. Xiao, Mitochondrial dysfunction and oxidative damage in the brain of diet-induced obese rats but not in dietresistant rats, Life Sci. 110 (2014) 53-60. doi:10.1016/j.lfs.2014.07.018.

[77] S.P. Brooks, S.B. Dunnett, Tests to assess motor phenotype in mice: a user's guide, Nat. Rev. Neurosci. 10 (2009) 519-529. doi:10.1038/nrn2652. [78] A.-L. Häcker, J.-L. Bigras, M. Henderson, T.A. Barnett, M.-E. Mathieu, Motor Skills of Obese and Severely Obese Children and Adolescents - A CIRCUIT Study, J. Strength Cond. Res. (2017) 1. doi:10.1519/JSC.0000000000002213.

[79] F. Passetti, The Frontal Cortex of the Rat and Visual Attentional Performance: Dissociable Functions of Distinct Medial Prefrontal Subregions, Cereb. Cortex. 12 (2002) 1254-1268. doi:10.1093/cercor/12.12.1254.

[80] S. Stranges, J.M. Dorn, P. Muti, J.L. Freudenheim, E. Farinaro, M. Russell, T.H. Nochajski, M. Trevisan, Body fat distribution, relative weight, and liver enzyme levels: a population-based study, Hepatology. 39 (2004) 754-63. doi:10.1002/hep.20149.

[81] J.C. Fraulob, R. Ogg-Diamantino, C. Fernandes-Santos, M.B. Aguila, C.A. Mandarim-de-Lacerda, A Mouse Model of Metabolic Syndrome: Insulin Resistance, Fatty Liver and Non-Alcoholic Fatty Pancreas Disease (NAFPD) in C57BL/6 Mice Fed a High Fat Diet, J. Clin. Biochem. Nutr. 46 (2010) 212-223. doi:10.3164/jcbn.09-83.

[82] C. Liedtke, T. Luedde, T. Sauerbruch, D. Scholten, K. Streetz, F. Tacke, R. Tolba, C. Trautwein, J. Trebicka, R. Weiskirchen, Experimental liver fibrosis research: update on animal models, legal issues and translational aspects, Fibrogenesis Tissue Repair. 6 (2013) 19. doi:10.1186/1755-1536-6-19. [83] M.V. Machado, G.A. Michelotti, G. Xie, T. Almeida Pereira, T.P. de Almeida, J. Boursier, B. Bohnic, C.D. Guy, A.M. Diehl, Mouse models of diet-induced nonalcoholic steatohepatitis reproduce the heterogeneity of the human disease, PLoS One. $10 \quad$ (2015) e0127991. doi:10.1371/journal.pone.0127991.

[84] G. Goliasch, F. Wiesbauer, H. Blessberger, S. Demyanets, J. Wojta, K. Huber, G. Maurer, M. Schillinger, W.S. Speidl, Premature myocardial infarction is strongly associated with increased levels of remnant cholesterol, J. Clin. Lipidol. 9 (2015) 801-806.e1. doi:10.1016/j.jacl.2015.08.009. 
[85] A. Varbo, J.J. Freiberg, B.G. Nordestgaard, Remnant Cholesterol and Myocardial Infarction in Normal Weight, Overweight, and Obese Individuals from the Copenhagen General Population Study, Clin. Chem. 64 (2018) 219230. doi:10.1373/clinchem.2017.279463.

[86] I. Pomytkin, J.P. Costa-Nunes, V. Kasatkin, E. Veniaminova, A. Demchenko, A. Lyundup, K.-P. Lesch, E.D. Ponomarev, T. Strekalova, Insulin receptor in the brain: Mechanisms of activation and the role in the CNS pathology and treatment, CNS Neurosci. Ther. (2018). doi:10.1111/cns. 12866 .

[87] I. San Mauro Martin, S. Sanz Rojo, E. Garicano Vilar, L. González Cosano, R. Conty de la Campa, J.A. Blumenfeld Olivares, Lifestyle factors, diet and attention-deficit/hyperactivity disorder in Spanish children - an observational study, Nutr. Neurosci. (2019) 1-10. doi:10.1080/1028415X.2019.1660486.

[88] S. Cortese, L. Tessari, Attention-Deficit/Hyperactivity Disorder (ADHD) and Obesity: Update 2016, Curr. Psychiatry Rep. 19 (2017) 4. doi:10.1007/s11920-017-0754-1.

[89] A.L. Howard, M. Robinson, G.J. Smith, G.L. Ambrosini, J.P. Piek, W.H. Oddy, ADHD Is Associated With a "Western" Dietary Pattern in Adolescents, J. Atten. Disord. $15 \quad$ (2011) 403-411. doi:10.1177/1087054710365990.

[90] B. Franke, G. Michelini, P. Asherson, T. Banaschewski, A. Bilbow, J.K. Buitelaar, B. Cormand, S. V. Faraone, Y. Ginsberg, J. Haavik, J. Kuntsi, H. Larsson, K.-P. Lesch, J.A. Ramos-Quiroga, J.M. Réthelyi, M. Ribases, A. Reif, Live fast, die young? A review on the developmental trajectories of ADHD across the lifespan, Eur. Neuropsychopharmacol. 28 (2018) 1059-1088. doi:10.1016/j.euroneuro.2018.08.001. 


\section{Supplementary Material}

Table 1. Diet composition for the Western diet and control diet used in the study. Western diet is characterized by increased content of fat, cholesterol and sucrose and high energy density.

\begin{tabular}{|c|c|c|}
\hline Diet & Western diet & Control diet \\
\hline \multicolumn{3}{|c|}{ Diet composition, \% } \\
\hline Fat & 21 & 3.5 \\
\hline Saturated & 12 & 0.6 \\
\hline Cholesterol & 0.2 & 0 \\
\hline Carbohydrates & 50 & 66 \\
\hline Sucrose & 35 & 6 \\
\hline Protein & 20 & 19 \\
\hline Fiber & 5 & 5 \\
\hline Minerals and vitamins & 4 & 6 \\
\hline \multicolumn{3}{|c|}{ Caloric Information, \% kcal } \\
\hline Fat & 43 & 9 \\
\hline Carbohydrate & 40 & 67 \\
\hline Protein & 17 & 24 \\
\hline $\begin{array}{l}\text { Energy } \\
\text { kcal/gm }\end{array}$ & 4.67 & 3.23 \\
\hline
\end{tabular}


Table 2. Sequences of the primers used in qPCR. Specific primer pairs were used in qPCR for measurement of serotonin transporter (Sert) and glyceraldehyde3-phosphate dehydrogenase (Gapdh) gene expression.

\begin{tabular}{|l|l|l|}
\hline Gene & Forward primer & Reverse primer \\
\hline Sert & TGCCTTTTATATCGCCTCCTAC & CAGTTGCCAGTGTTCCAAGA \\
\hline Gapdh & TGCACCACCAACTGCTTAG & GGATGCAGGGATGATGTTC \\
\hline
\end{tabular}

Feeding with Western diet and testing in glucose and insulin tolerance tests

Cohort 1 was subjected to a glucose tolerance test, and thus food deprivation, on day 23. The glucose tolerance test was performed on day 22 in cohort 2 , and, in cohort 3 , the glucose tolerance test was performed on day 17 and then the insulin tolerance test was performed on day 22. Thus, total exposure of the groups to experimental diet was approximately 22 days.

Feeding with Western diet impairs motor coordination in rotarod test on Day 19

Mice fed with CD or WD were tested in rotarod test on day 19 of dietary challenge. After 6 training trials, they were tested in the $7^{\text {th }}$ trial and the latency to fall was measured. The latency to fall was decreased in mice fed with WD compared to $\mathrm{CD}(\mathrm{U}=5.5, \mathrm{P}=0.0433$, Mann-Whitney test, Fig.S1), suggesting impaired motor functions. 


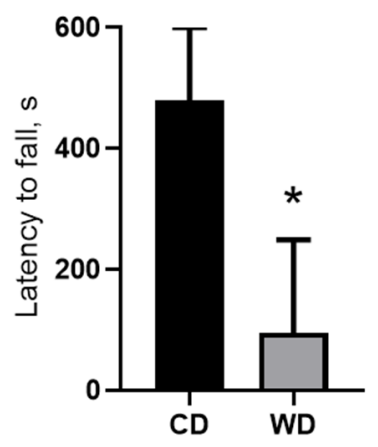

Figure S1. Feeding with Western diet impairs motor coordination in rotarod test on day 19. The latency to fall is decreased in the group fed with Western diet compared to control. CD - Control diet, WD - Western diet. * $\mathrm{P}<0.05$ compared to CD (Mann-Whitney test). Data are presented as median and interquartile range.
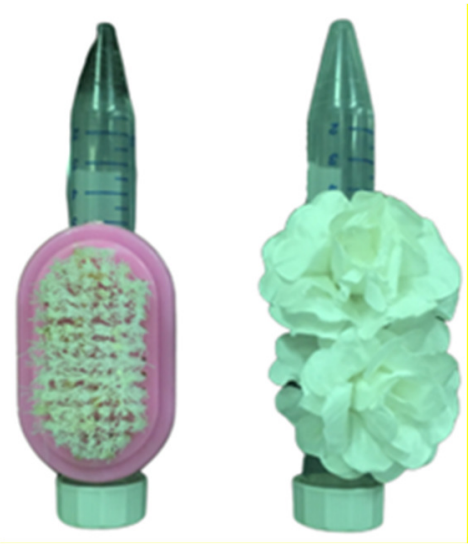

Figure S2. Objects used in Object exploration / recognition test. Two objects were similar in size but had different surface texture and shape. 


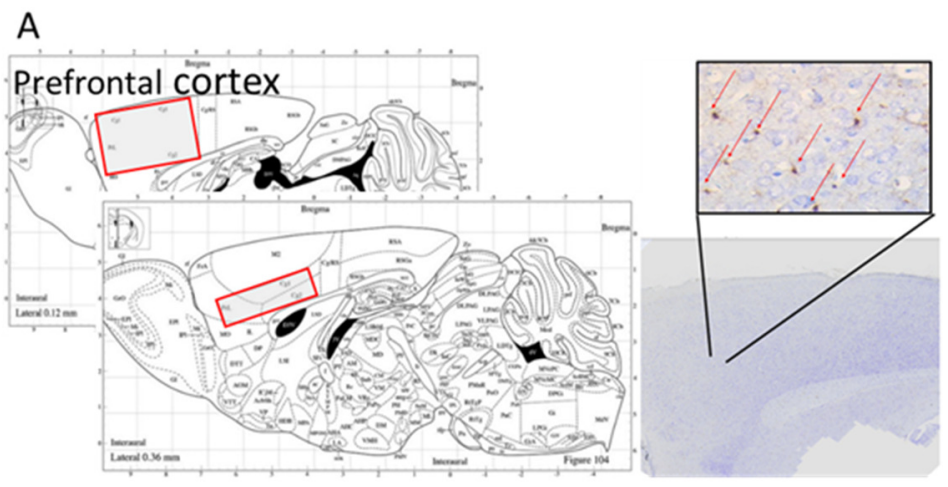

B
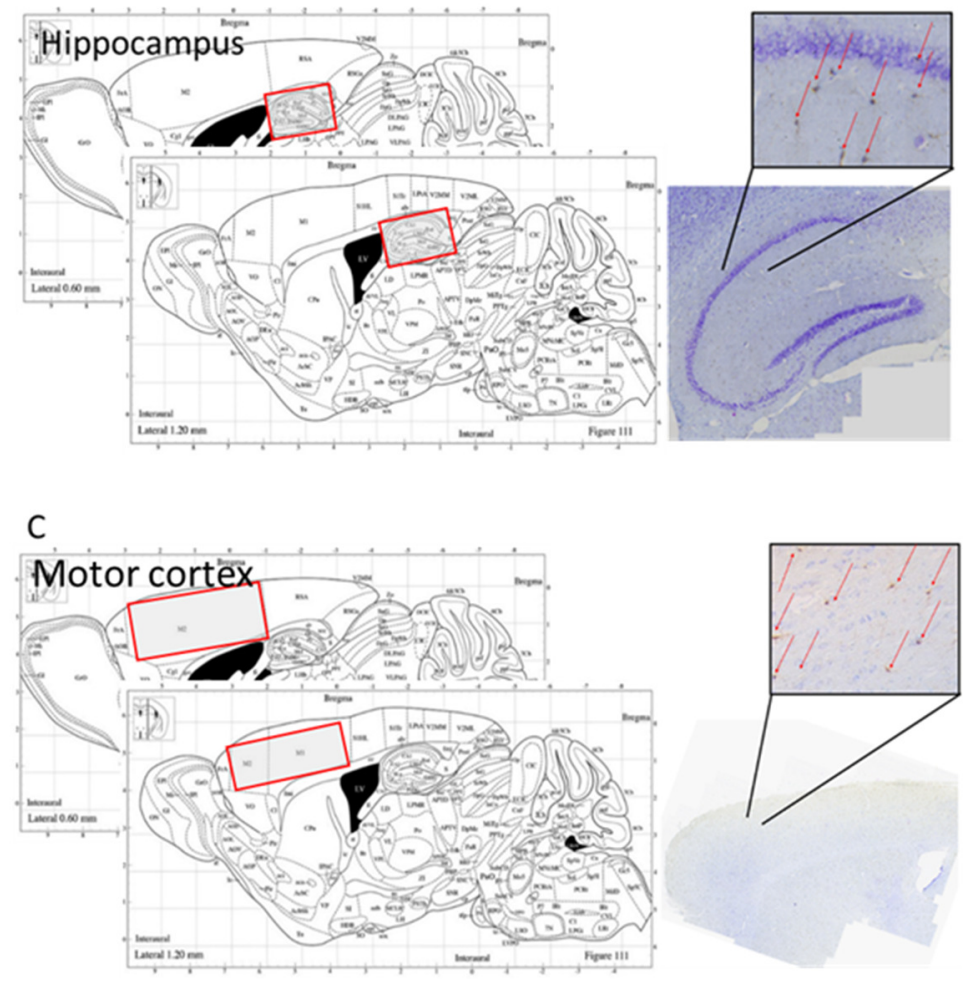

Figure S3. Coordinates of brain areas used for Iba-positive cell counting. (A) Prefrontal cortex, (B) hippocampus, (C) motor cortex were delineated according to the Paxinos and Franklin's the Mouse brain atlas. 
Effects of the Western diet on mouse body weight, weights of organs and visceral fat and diet and water intake

In body weight, no significant effect of the diet was found (Cohort 1: $\mathrm{F}=0.7261, \mathrm{P}=0.4108 ;$ Cohort 2: $\mathrm{F}=0.005017, \mathrm{P}=0.9458$; Cohort 3: $\mathrm{F}=0.2193, \mathrm{P}=0.6496$, two-way ANOVA). No differences between the groups fed WD or CD were found in body weight (Figs.S4A-C). No differences between the groups were found in the weights of liver, right and left kidneys, spleen, heart and visceral fat $(\mathrm{P}>0.05$, unpaired $\mathrm{t}$ test, Figs.S4D-I). No differences were found in daily diet intake in calories normalized per body weight and in daily water intake $(\mathrm{P}>0.05$, two-way ANOVA for repeated measures, Fig.S4J-K). 
A

Body weight, Cohort 1

B

Body weight, Cohort 2

C Body weight, Cohort 3
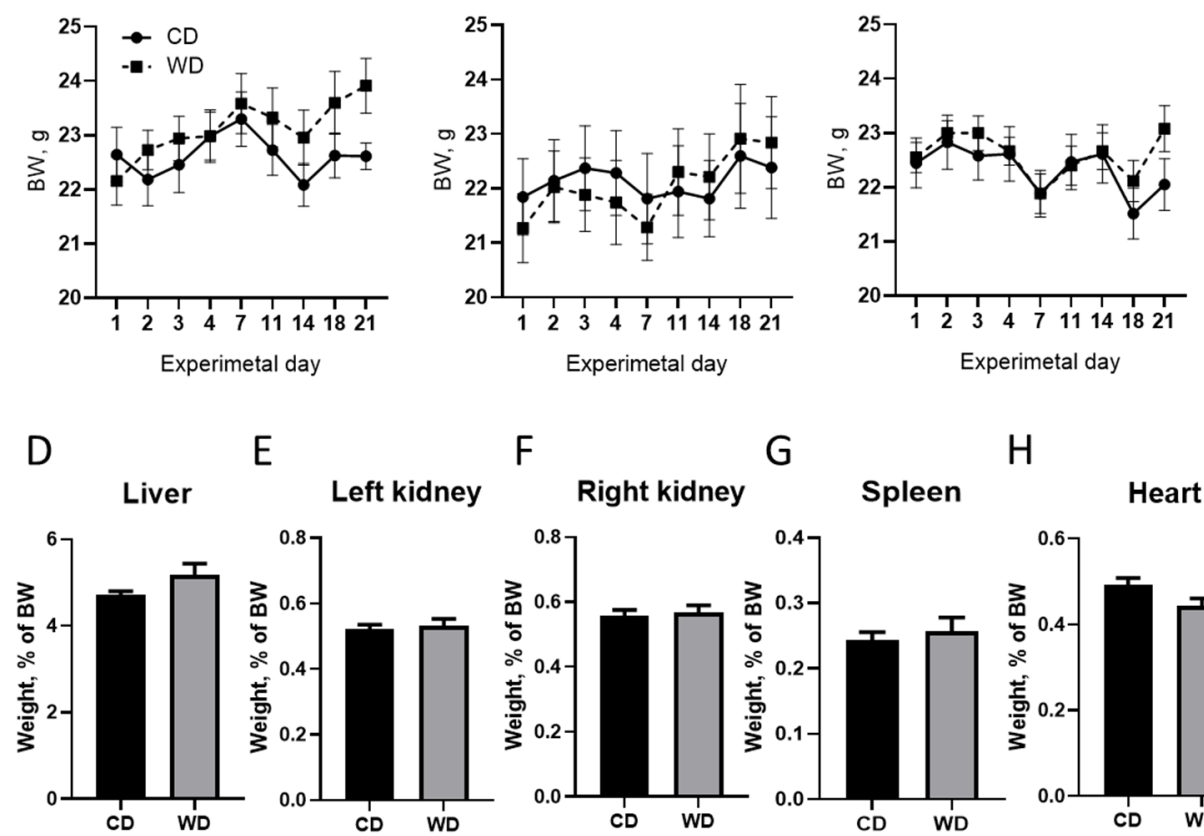

E Left kidney

F G

G $\quad H$

Heart
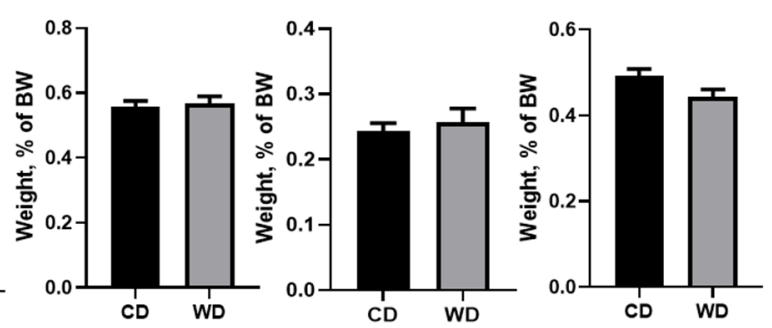

I

J

Visceral fat

Diet intake
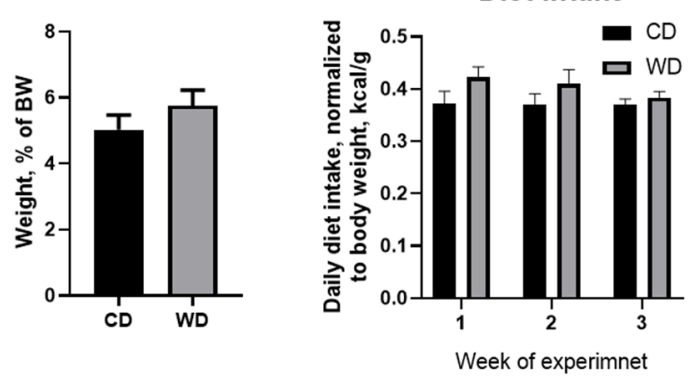

K

Water intake



Figure S4. Effects of 3-week housing on Western diet on mouse body weight, weights of organs and visceral fat and diet and water intake. Western diet feeding does not alter mouse (A-C) body weight, (D-I) organ and visceral fat weight or (J-K) diet and water intake. CD - Control diet, WD Western diet, BW - body weight. $\mathrm{P}>0.05$ compared to CD (two-way ANOVA or unpaired $\mathrm{t}$ test). Data are presented as Mean \pm SEM. 


\section{Effects of Western diet on fasting blood glucose and glucose tolerance on days 17 and 23 of dietary challenge}

In glucose tolerance tested on experimental day 23 (Cohort 1), two-way ANOVA revealed significant effects of diet x time interaction, diet and the time of after glucose load $(\mathrm{F}=3.905, \mathrm{P}=0.0053 ; \mathrm{F}=12.50$, $\mathrm{P}=0.0166$ and $\mathrm{F}=117.6, \quad \mathrm{P}<0.0001, \quad$ respectively, Fig.S5A). In comparison with control mice, WD-fed group showed a significant increase in blood glucose levels at the time point $10 \mathrm{~min}$ of the test $(\mathrm{P}<0.0001$, Sidak's multiple comparisons test), suggesting an impairment in glucose tolerance in the latter group. No difference between the groups was found in blood glucose after $18 \mathrm{~h}$ food deprivation on day $21(\mathrm{P}>0.05$, unpaired t test, Fig.S5B). In the glucose blood level measured in glucose tolerance test on day 17, a twoway ANOVA for repeated measures revealed no diet $\mathrm{x}$ time after glucose load interaction or effect of the diet $(\mathrm{F}=0.435, \mathrm{P}=0.8514$ and $\mathrm{F}=3.211, \mathrm{P}=0.1476$, respectively, Fig.S5C). Basal glucose level after $5 \mathrm{~h}$ food deprivation measured on Day 22 was increased in WD group $(\mathrm{t}=2.869, \mathrm{P}=0.0203$, unpaired $\mathrm{t}$ test, Fig.S5D). 
A

Glucose tolerance test D23 Cohort 1

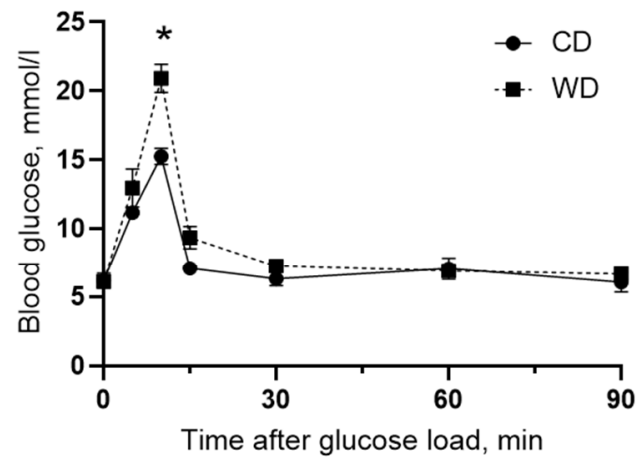

C Glucose tolerance test D17

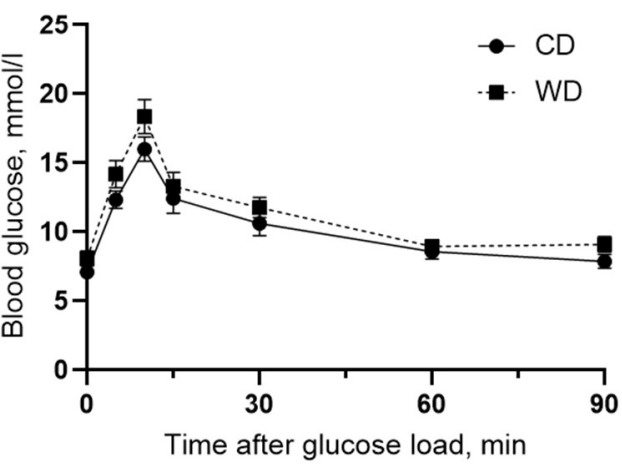

B

\section{Glucose level, $18 \mathrm{~h}$ food deprivation, D22}

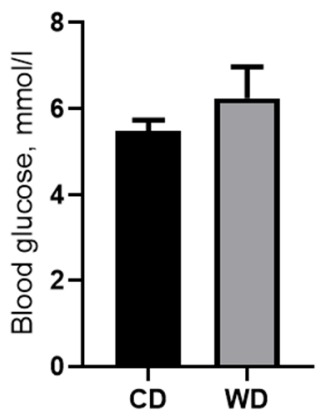

D

\section{Glucose level, $5 \mathrm{~h}$ food deprivation, D22}

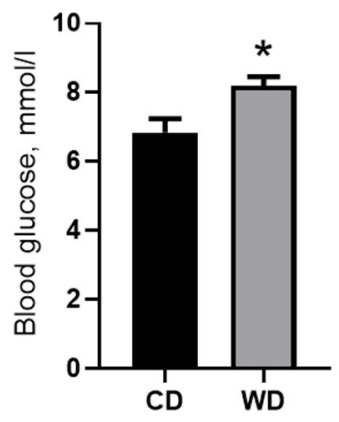

Figure S5. Effects of Western diet on fasting blood glucose and glucose tolerance on Day 23. (A) Glucose tolerance was decreased in WD group on day 23 in cohort 1. (B) Basal blood glucose level did not differ between the groups after $18 \mathrm{~h}$ of food deprivation. (C) No difference in glucose tolerance was found after 16 days of WD feeding. (D) Basal blood glucose level was increased in WDfed mice after 5 h food deprivation. CD- Control diet, WD - Western diet. * $\mathrm{P}<0.05$ compared to CD (unpaired t test or Sidak's multiple comparisons test). Data are presented as Mean \pm SEM. 
Effects of Western diet on latency to fall in inverted screen and duration of object exploration

No difference was found in the latency to fall in inverted screen test between the groups ( $\mathrm{P}>0.05$, Mann-Whitney test, Fig.S6A). In duration of object exploration, two-way ANOVA revealed significant diet $\mathrm{x}$ object interaction and effect of the object $(\mathrm{F}=14.40, \mathrm{P}=0.0016$; $\mathrm{F}=7.143, \quad \mathrm{P}=0.0167$, respectively, Fig.S6B). Post hoc analysis revealed increase in duration of new object exploration compared to familiar object in control group $(\mathrm{q}=6.467, \mathrm{P}=0.0016$, Tukey's multiple comparisons test) but not in WD group $(\mathrm{P}>0.05)$.

A

Inverted screen

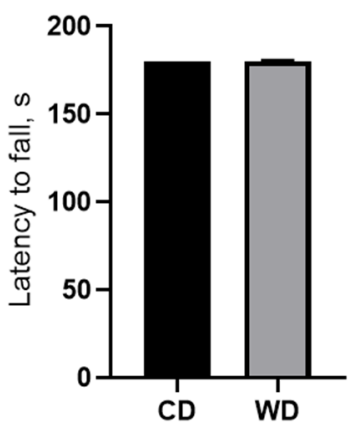

B

Object exploration

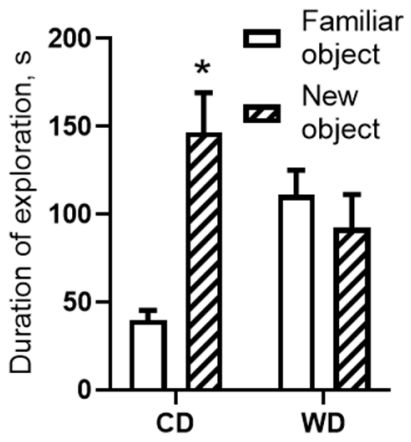

Figure S6. Effects of Western diet on latency to fall in inverted screen and duration of object exploration. (A) No difference between the groups was found in inverted screen test performance. (B) The duration of new object exploration compared to familiar object was increased in control group but not in WD group. CD - Control diet, WD - Western diet. * $-\mathrm{P}<0.05$ compared to CD (unpaired t test or Tukey's multiple comparisons test). Data are presented as Mean \pm SEM. 
Effects of Western diet on floating and climbing in forced swim test

On Day 1 of Forced swim test, latency to float, total duration of floating and duration of climbing behaviour did not differ between the groups ( $\mathrm{P}>0.05$, Fig.S7A-C). No difference was found in latency to float on day $2(\mathrm{t}=1.837, \mathrm{P}=0.0912$, unpaired $\mathrm{t}$ test, Fig.S7D). The total duration of floating on day 2 was increased in WD-fed mice compared to control ( $\mathrm{t}=2.924, \mathrm{P}=0.0148$, Fig.S7E). Duration of climbing behaviour on day 2 did not differ between the groups $(\mathrm{P}>0.05$, Fig.S7F $)$.

\section{Western diet does not alter locomotor activity in open field test}

To rule out possible confounds of altered general locomotion in analysis of other behavior, possible effects of WD on locomotor activity were studied in open field test. Mice were fed with $\mathrm{CD}$ or $\mathrm{WD}$, and one cohort (6 mice per group were used) was tested in open field on day 18 and another cohort (5 mice per group were used) was tested in open field on day 21. The open field apparatus consisted of a square arena $(50 \mathrm{~cm} \times 50 \mathrm{~cm})$. Mice were put in the center of the open field arena, and their behavior was video recorded for 10 min under a stress-free 5 Lux lighting. Distance travelled was analyzed off-line using the EthoVision software (Noldus, Wageningen, The Netherlands). No significant differences between the groups were found in distance 
travelled on either day 18 or day $21(\mathrm{t}=0.7482, \mathrm{P}=0.4837$ and $\mathrm{t}=0.0124, \mathrm{P}=0.9904$, respectively, unpaired t test, Figs.S8A-B). As same mice were tested on day 19 in a rotarod and showed signs of motor dyscoordination, taken together these data suggest that motor deficits induced by WD cannot be ascribed to altered locomotion (Fig.S1).

\section{Forced swim test D1}

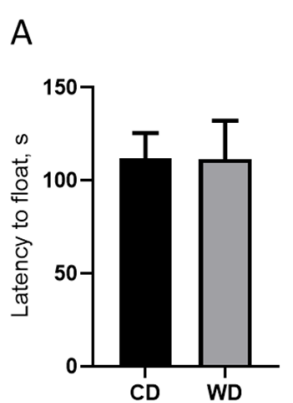

B

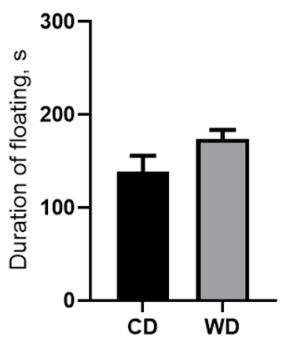

Forced swim test D2

D

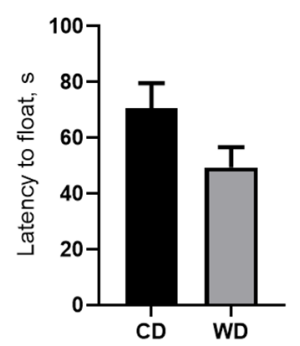

$\mathrm{E}$

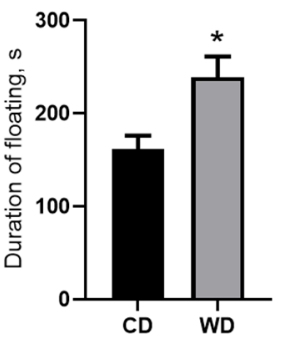

C

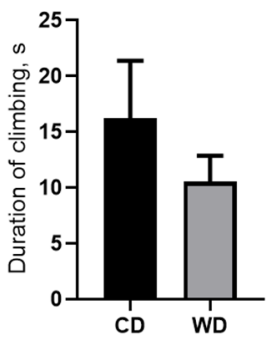

$\mathrm{F}$

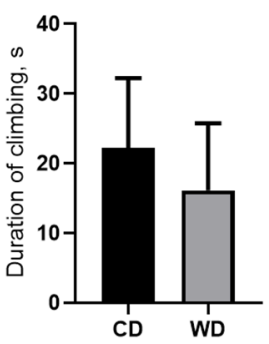

Figure S7. Effects of Western diet on floating and climbing in the swim test. On Day 1 of swim test, (A) latency to float, (B) total duration of floating and (C) duration of climbing behaviour did not differ between the groups. (D) On Day 2 of the swim test, latency to float did not differ between the groups. (E) Total duration of floating on Day 2 was increased in WD-fed mice compared to controls. (F) Duration of climbing behaviour on Day 2 did not differ between the groups. CD - Control diet, WD - Western diet. * - P $<0.05$ compared to CD (unpaired $\mathrm{t}$ test or Tukey's multiple comparisons test). Data are presented as Mean \pm SEM. 
A

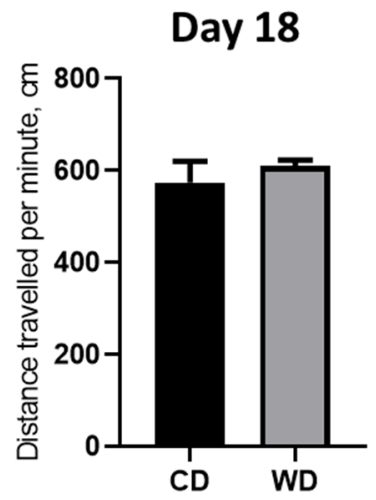

B

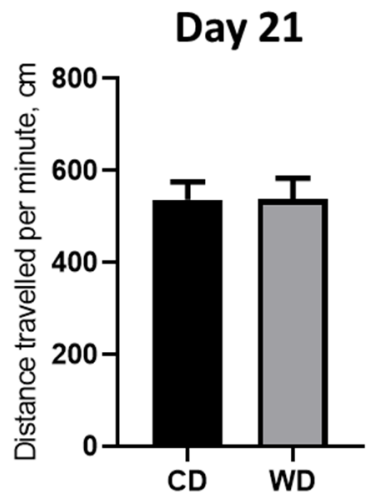

Figure S8. Western diet does not affect locomotor activity in open field test under 5 lux lighting. (A-B) No difference between the groups was found in distance travelled in open field on days 18 or 21 of dietary challenge. $\mathrm{CD}-$ Control diet, WD - Western diet. $\mathrm{P}>0.05$ (Unpaired t test). Data are presented as Mean \pm SEM. 


\section{Chapter 4}

Metabolic, molecular and behavioural effects of

Western diet in serotonin transporter-deficient mice: rescue by heterozygosity? 


\section{Chapter 4. Metabolic, molecular and behavioural effects of Western diet in serotonin transporter- deficient mice: rescue by heterozygosity?}

Ekaterina Veniaminova ${ }^{1,2}$, Raymond Cespuglio ${ }^{2,3}$, Irina Chernukha ${ }^{4}$, Angelika G. Schmitt-Boehrer ${ }^{5}$, Sergey Morozov ${ }^{6}$, Allan V. Kalueff ${ }^{7,8}$, Oxana Kuznetsova ${ }^{4}$, Daniel C. Anthony ${ }^{2,9}$, Klaus-Peter Lesch ${ }^{1,2,5}$, Tatyana Strekalova ${ }^{1,2,5,6}$

${ }^{1}$ Department of Psychiatry and Neuropsychology, School for Mental Health and Neuroscience, Maastricht University, Maastricht, The Netherlands; ${ }^{2}$ Laboratory of Psychiatric Neurobiology, Institute of Molecular Medicine, Sechenov First Moscow State Medical University, Moscow, Russia; ${ }^{3}$ Faculty of Medicine, Neuroscience Research Center of Lyon, C. Bernard University Lyon 1, Lyon, France; ${ }^{4}$ V.M. Gorbatov Federal Research Center for Food Systems of RAS, Moscow, Russia; ${ }^{5}$ Division of Molecular Psychiatry, Center of Mental Health, University of Würzburg, Würzburg, Germany; ${ }^{6}$ Institute of General Pathology and Pathophysiology, Moscow, Russia; ${ }^{7}$ School of Pharmacy, Southwest University, Chongqing, China; ${ }^{8}$ Institute of Translational Biomedicine, St. Petersburg State University, St. Petersburg, Russia; ${ }^{9}$ Department of Pharmacology, Oxford University, Oxford, UK

Veniaminova E, Cespuglio R, Chernukha I, Schmitt-Boehrer AG, Morozov S, Kalueff AV, Kuznetsova O, Lesch KP, Anthony DC, Strekalova T. Metabolic, molecular and behavioural effects of Western diet in serotonin transporter-deficient mice: rescue by heterozygosity? Frontiers in Neuroscience. Under revision. 


\section{Abstract}

Reduced function of the serotonin transporter (SERT) is associated with increased susceptibility to anxiety and depression, and with type-2 diabetes, which is especially true in older women. Preference for a "Western diet" (WD), enriched with saturated fat, cholesterol and sugars, may aggravate these conditions. In previous studies, decreased glucose tolerance, central and peripheral inflammation, dyslipidaemia, emotional, cognitive and social abnormalities were reported in WD-fed young female mice. We investigated the metabolic, molecular, and behavioural changes associated with a three-week long dietary regime of either the WD or control diet in 12-month-old female mice with three different Sert genotypes: heterozygous (Sert+/-: HET), homozygous (Slc6a4) gene knock out (Sert-/-: KO) or wild-type mice $($ Sert $+/+$ : WT). In the WT-WD and KO-WD groups, but not in HET-WD-fed mice, most of changes induced by the WD paralleled those found in the younger mice, including brain overexpression of inflammatory marker toll-like receptor 4 ( $\left.T l r_{4}\right)$, impaired hippocampus-dependent performance in the marble test. However, the 12-month-old female mice became obese. Control diet $\mathrm{KO}$ mice exhibited impaired hippocampal-dependent behaviours, increased brain expression of the serotonin receptors Htr2c and Htr1b, as well as increased Tlr4 and mitochondrial regulator, peroxisome proliferator-activated receptor gamma-coactivator-1a 
(Ppargc1a). Paradoxically, these, and other changes, were reversed in KO-WD mutants, suggesting a complex interplay between Sert deficiency and metabolic factors as well as potential compensatory molecular mechanisms that might be disrupted by the WD exposure. Most, but not all, of the changes in gene expression in the brain and liver of KO mice were not exhibited by the HET mice fed with either diet. Some of the WD-induced changes were similar in the KO-WD and HET-WD-fed mice, but the latter displayed a "rescued" phenotype in terms of diet-induced abnormalities in glucose tolerance, neuroinflammation and hippocampus-dependent performance. Thus, complete versus partial Sert inactivation in aged mice results in distinct metabolic, molecular and behavioural consequences in response to the WD. Our findings show that Sert $+/-$ mice are resilient to certain environmental challenges and support the concept of heterosis as evolutionary adaptive mechanism.

\section{Keywords}

Sert-deficient mice, Western diet, aging, glucose tolerance, Tolllike receptor 4 (TLR4), serotonin receptors, obesity, heterosis. 


\section{Introduction}

Serotonin transporter (SERT), a key element of serotonergic neurotransmission (Collier et al., 1996; Murphy et al., 2004), is also involved in the regulation of metabolic processes (Giannaccini et al., 2013; Pomytkin et al., 2015, 2018; Stuart and Baune, 2012). In humans, a variant of the upstream regulatory region of the SERT (SLC6A4) gene, the so-called short (s) allele, in comparison with long (l) allele is associated with lower SERT activity and stressed-related vulnerability to anxiety and depression (Caspi et al., 2010; Greenberg et al., 2000; Lesch et al., 1996), and also with higher body mass index (BMI) (Fuemmeler et al., 2008; Sookoian et al., 2007) and incidence of type2 diabetes (Iordanidou et al., 2010), which are typical for the female sex and aging (Batsis and Zagaria, 2018; Kautzky-Willer et al., 2016; Khabazkhoob et al., 2017).

Individuals with metabolic syndrome and obesity display decreased SERT expression in the brain and periphery (Giannaccini et al., 2013; Nam et al., 2018). Excessive intake of a hypercaloric diet, enriched with saturated fat and sugars, has been shown to suppress the binding of hypothalamic SERT in obese subjects and in insulinresistant subjects, that is independent of body weight gain (Koopman et al., 2013; Versteeg et al., 2017). Thus, diminished SERT activity is believed to underlie negative changes associated with metabolic syndrome (Stuart and Baune, 2012) and in turn, metabolic 
abnormalities resulting in reduced SERT function that can contribute to emotional disturbances (Pomytkin et al., 2015, 2018). However, the molecular mechanisms of the interplay between genetic SERT deficiency and metabolic dysregulation remain unclear.

Animal studies support the observations made in humans concerning the relationship between SERT deficiency and diabetes-like metabolic changes. Sert-deficient mice (Sert/-: KO) have been reported to display decreased glucose tolerance, increased deposition of white adipose tissue that increases with aging, and late-onset obesity; these changes were particularly marked in females (Chen et al., 2012; Murphy and Lesch, 2008; Üçeyler et al., 2010; Zha et al., 2017).

Aging is well known to be associated with compromised metabolic function (Boemi et al., 2016) and changes in the serotonergic regulation (Rodríguez et al., 2012). Abnormal distribution of fat in the elderly increases the risk or exacerbates the negative effects of obesity on metabolic function, including a decline in insulin sensitivity and glucose tolerance (Karakelides et al., 2010; Morita et al., 2006). In humans, each decade results in a $10 \%$ decrease in the density of SERT binding sites in the brain stem and thalamus (Yamamoto et al., 2002); this decrease is also found in monkeys and mice (Herrera-Pérez et al., 2013; Kakiuchi et al., 2001). Aging also results in a decrease in circulating serotonin and alterations in the densities of the brain serotonin 
receptors 5-HT1A, 5-HT2A, 5-HT1B (Matuskey et al., 2012; Meltzer et al., 1998) that are more profound in women (Meltzer et al., 1998).

Despite the evidence of a relationship between genetic SERT deficiency and diabetes-like metabolic conditions, little is known about the underlining molecular mechanisms, and few studies have addressed this issue. In the human population, the combination of genetic SERT deficiency, increased intake of Western diet (WD), aging is a widespread, but the interactions are difficult to explore owing to the presence of so many potentially confounding factors. Mutant animals provide an opportunity to model the interactions between genotype and diet at different ages and in different sexes. The majority of studies addressing the impact of decreased SERT function on metabolic regulation during hypercaloric dietary challenge have been performed with young Sert-deficient mice. For example, Chen et al. (2012) reported the presence of elevated fasting glucose levels, impaired glucose tolerance and insulin resistance in Sert-/- animals exposed to a high-fat diet for 3 months in male mice. Sert/- female rats, but not male animals, demonstrated increased abdominal fat when fed either standard chow or a diet with high in fat and sugar content (Homberg et al., 2010).

Concerning the effects of the Western diet and aging on the metabolic characteristics of Sert heterozygous (Sert ${ }^{+/-}$: HET) animals, very limited literature is available. It has been reported that diet- 
induced metabolic changes in young SERT heterozygous mice are intermediate in their magnitude, showing impaired glucose tolerance and insulin resistance, with respect to the changes in the wild type and knockout phenotypes (Chen et al., 2012). However, HET mice are considered to be qualitatively distinct from Sert ${ }^{/-}$animals and closer mimic of the short allele human condition, displaying allelic variation of SERT function. Substantial differences between two genotypes have been described in a maternal separation and other stress models, in which the HPA axis was suggested not to be effect in the same way in HET and KO animals (Jiang et al., 2009; van der Doelen et al., 2014). In a prenatal stress model, HET mice demonstrated signs of superior stress resilience compared to WT, displaying reduced scores of anxietylike behaviour and improved cognitive performance (van den Hove et al., 2011), while other studies showed increased stress reactivity in KO (Bearer et al., 2018; Wellman et al., 2007).

In the present study we sought to investigate metabolic, molecular and behavioural changes induced by the WD in aged mice with complete or partial genetic SERT deficit. We employed a previously validated model that involves feeding mice with the WD for three weeks and we evaluated metabolic and neurobiological hallmarks of the WD-induced induced syndrome in vitro and ex vitro assays (Strekalova et al., 2015, 2016; Veniaminova et al., 2016, 2017, 2020). In this model, impaired glucose tolerance, increases in cholesterol and 
leptin blood levels, brain and liver over-expression of toll-like receptor 4 (Tlr4), decreased expression of mitochondrial markers peroxisome proliferator-activated receptor gamma coactivator 1 (Ppargc1) a and b and decreased Sert expression in the brain are all features. These molecular changes are accompanied by depressive- and anxiety-like behaviours, signs of impulsivity, lowered sociability and cognitive deficits (Strekalova et al., 2015, 2016; Veniaminova et al., 2016, 2017, 2020). Here, in view of the changes observed in 5HT receptor densities in aging and in relation to SERT deficiency, we also studied gene expression of serotonin receptors Htr1a, Htr1b and Htr2a in the brain. The expression of the serotonin receptors 5-HT2C and 5-HT6 were also owing to their know role in the regulation of emotionality and metabolic function (Bickerdike, 2003; Heal et al., 2008; Millan, 2005; Wesołowska, 2010).

\section{$2 \quad$ Methods}

\subsection{Animals}

Experiments were performed using 12-month-old homozygous Sert ${ }^{--}$and heterozygous $\mathrm{Ser}^{+/-}$female mice and wild type littermates born from heterozygous mutants at the tenth generation (F10) of backcrossing with C57BL/6J mice, all genotypes were confirmed by PCR. Mice were housed 3-4 per cage during the study, under a reversed $12 \mathrm{~h}$ light-dark cycle (lights on: 21:00 h) with food and water ad libitum 
and under controllable laboratory conditions $\left(22 \pm 1^{\circ} \mathrm{C}, \quad 55 \%\right.$ humidity). Laboratory housing conditions and experimental procedures were set up and maintained in accordance with the European Communities Council Directive for the care and use of laboratory animals (2010/63/EU) and approved by the local ethics committees of C. Bernard University and MSMU (\#11-18).

\subsection{Study design and diets}

Mice were fed with a standard laboratory diet (control diet, CD) with an energy content of $3.8 \mathrm{kcal} / \mathrm{g}, 4.3 \%$ of fat (1.3 of saturated fat) (D18071801, Research Diet Inc., New Brunswick, NJ, USA) or with a diet containing $0.2 \%$ cholesterol, $21.3 \%$ of fat (10.5\% of saturated fat), and an energy content of $4.6 \mathrm{kcal} / \mathrm{g}$, Western diet (D11012302, Research Diet Inc., New Brunswick, NJ, USA) for three weeks as described elsewhere (Strekalova et al., 2015, 2016; Veniaminova et al., 2017). The content of the nutrients in calories and weight and the ingredients are indicated in Supplementary Table S1. Body weight and intake of diet and water were monitored weekly (on day 1, day 8, day 15 and day 21). Daily intake of calories and water was normalized to body weight.

After a three-week period of dietary challenge, a cohort of mice was studied in novel cage, O-maze test, depressive-like behaviours in tail suspension and forced swim tests, in the pellet displacement tube 
(marble) test, a rodent paradigm for a hippocampus-dependent performance (Deacon et al., 2002; Strekalova and Steinbusch, 2010) and a glucose tolerance test (Fig. 1A). Another cohort of animals was exposed to the same dietary conditions and sacrificed and dissected for the analysis of gene expression (Fig. 1B). 6-7 mice per group were used in each study.

A

$\begin{array}{lllll}\text { Day } 1 & \text { Day } 20 & \text { Day } 21 & \text { Day } 22 & \text { Day } 23\end{array}$ Start of the diet: Control diet or Western diet

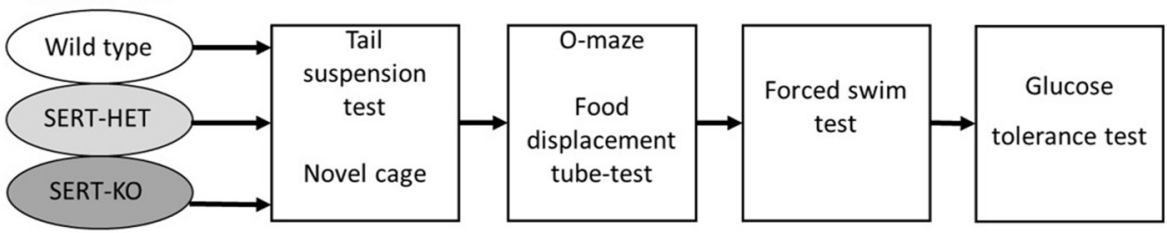

B

\section{Day 1}

Day 22

Start of the diet:

Control diet or Western diet

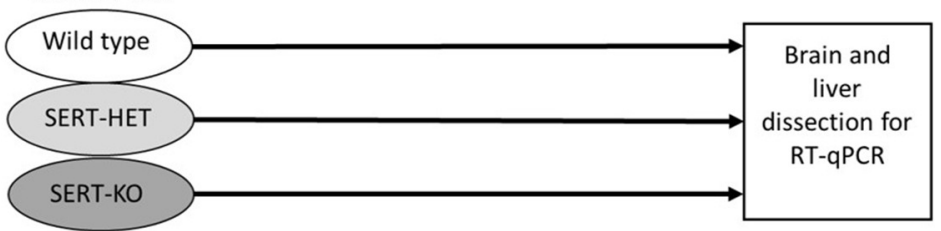

Figure 1. Experimental design. (A) Study of the effects of 3-week WD feeding of WT, HET and KO mice on behaviour in tail suspension, novel cage, O-maze, food displacement test, forced swim test, glucose tolerance test. (B) Study of the effects of 3-week WD feeding of WT, HET and KO mice on gene expression in the brain and liver. 


\subsection{Behavioural testing}

All behavioural tests were carried out during an active period of animals' light cycle (09:00-21:00); behaviour was recorded and scored offline. The experimenter was blind for the diet used and the genotype.

\subsubsection{Novel cage test}

The novel cage test was performed to assess exploration of a new environment. Mice were introduced into a standard plastic cage $(21 \times 27 \times 14 \mathrm{~cm})$, under 5 Lux lighting. The number of exploratory rears was counted for the first minute of the test by visual observation, as described elsewhere (Costa-Nunes et al., 2015).

\subsubsection{Elevated O-maze}

The maze consisted of a black circular path (runway width $5.5 \mathrm{~cm}$, diameter $46 \mathrm{~cm}$ ) that was placed $20 \mathrm{~cm}$ above the floor. Illumination intensity was 5 Lux. Two opposing compartments were protected by the walls (height $10 \mathrm{~cm}$ ). Mice were introduced to one of the two closed compartments. Latency to exit to the anxiety-related open compartments of the maze was scored, as described elsewhere (Strekalova et al., 2015).

\subsubsection{Tail suspension test}

Mice were subjected to the tail suspension by being hung by their tails with adhesive tape to a rod $50 \mathrm{~cm}$ above the floor for $6 \mathrm{~min}$, as described elsewhere (Malatynska et al., 2012). The lighting intensity 
on the height of the mouse position was 25 Lux. The trials were recorded by a video camera positioned directly in front of the mice while the experimenter observed the session from a distance in a dark area of the experimental room. The latency of the first episode of immobility was scored. In accordance with the commonly accepted criteria of immobility, the immobility behaviour was defined as the absence of any movements of the animals' head and body. The scoring method was previously validated using CleverSys software (CleverSys, Reston, VA, USA) and Noldus software (Wageningen, The Netherlands) (Malatynska et al., 2012).

\subsubsection{Swim test}

This test was carried out as described previously (Strekalova et al., 2015). Mice were placed into a plastic transparent cylinder (Ø $17 \mathrm{~cm})$ filled with water $\left(+23^{\circ} \mathrm{C}\right.$, water height $13 \mathrm{~cm}$, and height of cylinder $20 \mathrm{~cm}$ ) under the red lighting. Total duration of floating behaviour, defined by the absence of any directed movements of animals' head and body, was scored offline during the 6-min period. Latency to float was evaluated as well. The scoring method was previously validated using CleverSys software (CleverSys, Reston, VA, USA) and Noldus software (Wageningen, The Netherlands) (Malatynska et al., 2012). 


\subsubsection{Pellet displacement tube (marble) test}

All experimental groups were tested for pellet displacement in a tube test as described elsewhere (Deacon et al., 2002; Strekalova and Steinbusch, 2010). A tendency to displace small objects, for example, small stones or food pellets from a tube inside the cage, is speciesspecific in mice and has been demonstrated to depend on an intact hippocampal formation (Deacon et al., 2002). Using a paper tube (internal diameter $4 \mathrm{~cm}$, length $10 \mathrm{~cm}$ ), filled with 20 food pellets and placed in the cage $(21 \times 27 \times 14 \mathrm{~cm})$, the time required for $50 \%$-emptying of tube was recorded.

\subsection{Glucose Tolerance Test}

Oral glucose tolerance test (OGTT) was performed as described elsewhere (Veniaminova et al., 2017). The test mice were fasted overnight for $18 \mathrm{~h}$, beginning at 1600 . Thereafter, a glucose solution $(2 \mathrm{~g} / \mathrm{kg}, 1.8 \mathrm{~g} / \mathrm{l})$ was delivered by oral gavage and blood was sampled from the tail vein. Samples were obtained prior to glucose administration at time point 0 and 5, 15, 30, 60, 90 min afterwards. The level of blood glucose was analyzed using the OneTouch UltraEasy glucometer and strips (LifeScan OneTouch, Dubai, UAE). Fasting blood glucose concentrations and the area under a curve (AUC) for the whole test period and for the period between 60 and 90 min after glucose load were analyzed. 


\subsection{Tissue dissection}

Mice were terminally anaesthetized with isoflurane inhalation for a subsequent material collection. The brain of each mouse was perfused with saline and dissected, and dorsal raphe region zone, hypothalamus, hippocampus and prefrontal cortex were isolated and stored at $-80^{\circ} \mathrm{C}$ until use as described elsewhere (Couch et al., 2013).

\subsection{RNA extraction and qRT-PCR}

Total mRNA was extracted by using RNeasy Mini Kit (Qiagen, Venlo, the Netherlands). First-strand cDNA synthesis was performed using High-Capacity cDNA Reverse Transcription Kit (Applied Biosystems, Waltham, MA, USA); $1 \mu \mathrm{g}$ total RNA was converted into cDNA. Quantitative PCR for the genes of interest (Htr1a, Htr1b, Htr2a, Htr2c, Htr6, Ppargc1a, Ppargc1b, Tlr4) and the reference genes (glyceraldehyde 3-phosphate dehydrogenase (Gapdh), beta-actin (Actb), beta-2 microglobulin $(B 2 m))$ was performed using the SYBR Green PCR Master Mix (Applied Biosystems) and QuantStudio 7 Flex Real-Time PCR System (Applied Biosystems). Sequences of primers used are indicated in Supplementary Table S2. Reference genes for normalization were tested for stability using RefFinder software. Results of qRT-PCR measurement were expressed as Ct values and the comparative $\mathrm{Ct}$ method was used. Data are given as expression 
folds compared to the mean expression values in WT mice fed control diet as described elsewhere (Couch et al., 2013).

\section{$2.8 \quad$ Statistics}

Data were analyzed using GraphPad Prism version 8.01 (San Diego, CA, USA). For comparison of six groups, two-way ANOVA followed by Tukey's post-hoc test was used. One-way ANOVA followed by post-test for trend was used for analysis of genotype body weight data at baseline. For comparison of a group mean with $100 \%$, one sample $t$ test was performed. Three-way ANOVA analysis was performed in IBM SPSS Statistics 23 (Armonk, NY, USA). The level of significance was set at $\mathrm{p}<0.05$. Data were presented as Mean $\pm \mathrm{SEM}$ or Mean.

\section{$3 \quad$ Results}

\subsection{Western diet and SERT deficiency affect metabolic parameters}

There was a significant difference in body weight at baseline between the Sert genotypes $(\mathrm{F}=4.547, \mathrm{p}=0.016$, one-way ANOVA). A linear increase in body weight from WT to KO group $(\mathrm{p}=0.005$, post-test for trend; Fig. 2A) was found. Two-way ANOVA revealed a significant effect of the diet type on the body weight measured both in absolute values and normalized to basal values $(\mathrm{p}<0.05$, Table 1) after three weeks of feeding as measured on day 21 . 


\section{Body weight}

A

B

Day 1

Day 21

C

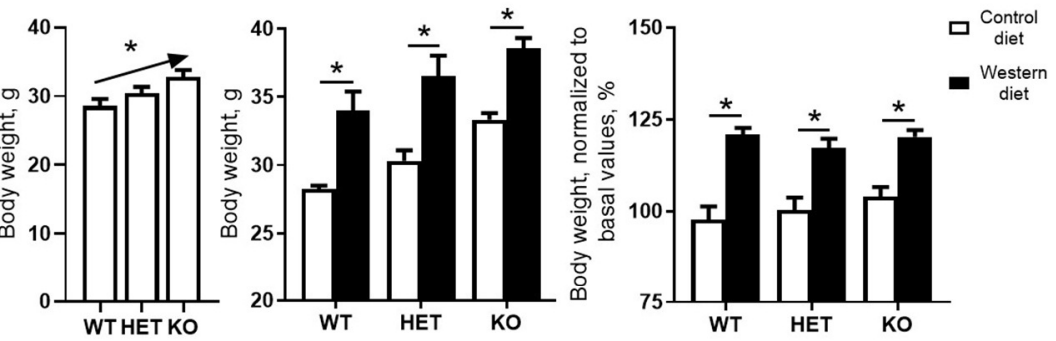

D



Food and water intake

E

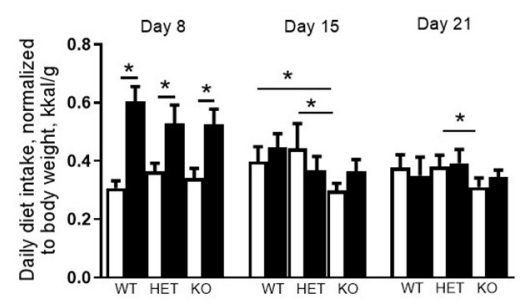

$\mathbf{F}$

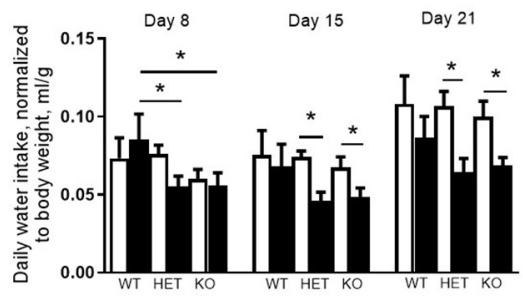

Figure 2. Effects of Western diet feeding on body weight and food and water intake. (A) Basal body weight of mice from different Sert genotypes: WT, HET, KO. (B-C) Body weight after 3-week housing on WD or CD in absolute values and normalized to basal body weight. (D) Dynamics of the mouse body weight measured every week during the experiment. Mice fed with WD demonstrated increase in body weight during the study. (E-F) Diet and water intake. ${ }^{*} \mathrm{p}<0.05$, one-way ANOVA and post-test for trend (A) or two-way ANOVA and Tukey's test (B-F), 6-7 animals per group were used. Data are shown as mean \pm SEM (A-C,E-F) or mean (D). 
The body weight of WT, HET and KO mice fed with WD was increased compared with the respective genotype-matched control groups (absolute body weight values: $\mathrm{p}=0.010, \mathrm{p}=0.001$, and $\mathrm{p}=0.005$, respectively, Tukey's test; normalized to basal values: $\mathrm{p}<0.001$, $\mathrm{p}<0.001$, and $\mathrm{p}<0.001$, respectively, Tukey's test; Figs. 2B-C). A significant interaction between the diet type and the day of the experiment and a significant effect of the genotype were found for the dynamics of body weight $(\mathrm{F}=6.452, \mathrm{p}<0.001$ and $\mathrm{F}=28.827, \mathrm{p}<0.001$, three-way ANOVA). No differences between the groups were found on days 1 and 8. On day 15, body weight was increased in HET-WD mice compared to control HET mice ( $\mathrm{p}=0.014$, Tukey's test; Fig. 2D); on day 21, all Sert genotype groups fed with WD displayed increased body weight compared to controls ( $\mathrm{p}=0.044$ for WT-WD, $\mathrm{p}=0.002$ for HET-WD, $\mathrm{p}=0.020$ for KO-WD, Tukey's test). Thus, 3-week WD feeding resulted in body weight increase in all Sert genotypes.

As revealed by three-way ANOVA, there was a significant interaction between genotype, diet and experimental day for daily diet calorie intake $(\mathrm{F}=5.944, \mathrm{p}<0.001)$. During the $1^{\text {st }}$ week of the experiment, daily calorie intake was increased in WT, HET and KO mice fed with WD compared to mice fed with CD $(\mathrm{p}<0.001, \mathrm{p}<0.001$, and $\mathrm{p}<0.001$, respectively, Tukey's test; Fig. 2E). Then, during the $2^{\text {nd }}$ week, calorie intake was decreased in $\mathrm{KO}$ mice fed with $\mathrm{CD}$ compared to WT and HET fed with CD $(\mathrm{p}=0.027$ and $\mathrm{p}=0.030$, 
respectively, Tukey's test). During the $3^{\text {rd }}$ week, calorie intake was decreased in KO-CD mice compared to HET-CD ( $\mathrm{p}=0.0231$, Tukey's test). Similar results were obtained for daily diet intake measured in grams per kg of body weight (Supplementary Fig. S1). Significant interaction between genotype and diet and experimental day and diet was found for daily water intake $(\mathrm{F}=15.487, \mathrm{p}<0.001$ and $\mathrm{F}=20.020$, $\mathrm{p}<0.001$, respectively, three-way ANOVA). During the $1^{\text {st }}$ week, water intake was decreased in HET-WD and KO-WD mice compared to WT-WD ( $\mathrm{p}=0.016$ and $\mathrm{p}=0.018$, respectively, Tukey's test; Fig. 2F $)$.

Table 1. Two-way ANOVA results for statistical analysis of metabolic parameters.

\begin{tabular}{|l|l|l|l|l|l|l|}
\hline Metabolic parameters & $\begin{array}{l}\text { Interaction, } \\
F\end{array}$ & $\begin{array}{l}\text { Interaction, } \\
p\end{array}$ & $\begin{array}{l}\text { Genotype, } \\
F\end{array}$ & $\begin{array}{l}\text { Genotype, } \\
p\end{array}$ & $\begin{array}{l}\text { Diet, } \\
F\end{array}$ & $\begin{array}{l}\text { Diet, } \\
p\end{array}$ \\
\hline Parameter & 0.09742 & 0.9075 & 8.510 & $\mathbf{0 . 0 0 1 2}$ & 37.98 & $<\mathbf{0 . 0 0 1}$ \\
\hline $\begin{array}{l}\text { Body weight } \\
\text { on D21, } \\
\text { absolute } \\
\text { values }\end{array}$ & 0.8320 & 0.4450 & 0.9820 & 0.3863 & 70.65 & $<\mathbf{0 . 0 0 1}$ \\
\hline $\begin{array}{l}\text { Body weight } \\
\text { on D21, } \\
\text { normalized to } \\
\text { basal values }\end{array}$ & 1.897 & 0.1710 & 7.369 & $\mathbf{0 . 0 0 3 0}$ & 6.514 & $\mathbf{0 . 0 1 7 2}$ \\
\hline $\begin{array}{l}\text { Fasting blood } \\
\text { glucose level }\end{array}$ & 1.343 & 0.2808 & 1.053 & 0.3651 & 9.242 & $\mathbf{0 . 0 0 5 8}$ \\
\hline $\begin{array}{l}\text { AUC for } \\
\text { glucose } \\
\text { tolerance test }\end{array}$ & 2.392 & 0.1139 & 0.3612 & 0.7007 & 9.357 & $\mathbf{0 . 0 0 6}$ \\
\hline $\begin{array}{l}\text { AUC for } \\
\text { glucose } \\
\text { tolerance test, } \\
\text { 60-90 min }\end{array}$ & & & & & & \\
\hline
\end{tabular}

$\mathrm{F}$ and $\mathrm{p}$ values are shown for interaction between genotype and diet, for genotype effect and for diet effect. 
During the $2^{\text {nd }}$ and $3^{\text {rd }}$ weeks, HET and KO groups fed with WD showed decreased water intake compared to the respective genotypematched groups fed with CD (HET-WD: $\mathrm{p}<0.001$ and $\mathrm{p}<0.001$, KO-WD: $\mathrm{p}=0.0003$ and $\mathrm{p}<0.0001$, respectively, Tukey's test). That way, Sert deficiency resulted in a decreased diet intake in KO mice and decreased water intake in HET and $\mathrm{KO}$ after WD exposure.

We found significant effects of the diet type and the genotype (two-way ANOVA, $\mathrm{p}<0.05$, Table 1 ) on the fasting blood glucose levels. Glucose levels after 18 hours of food deprivation were decreased in $\mathrm{KO}$ mice fed with WD compared to $\mathrm{KO}$ fed with $\mathrm{CD}(\mathrm{p}=0.024$, Tukey's test; Fig. 3A). Fasting blood glucose levels normalized to the respective genotype-matched CD group values were also decreased in KO-WD compared to $100 \%(\mathrm{t}=3.528, \mathrm{p}=0.0243$, one sample $\mathrm{t}$ test; Fig. 3B). Two-way ANOVA analysis demonstrated a significant diet effect $(\mathrm{p}<0.05$, Table 1; Fig. $\mathbf{3 C}$ ) on the area under the curve (AUC) calculated for glucose tolerance curve (Fig. 3D). Post-hoc analysis revealed a significant increase in AUC in WT and $\mathrm{KO}$ mice fed with WD compared to the respective control groups $(\mathrm{p}=0.021$ and $\mathrm{p}=0.028$, respectively, Tukey's test). No differences in AUC were found between HET-CD and HET-WD groups. There was a significant diet effect on AUC calculated for the period between 60 and 90 min after glucose load ( $<<0.05$, Table 1). This parameter was significantly increased in KO-WD compared to KO-CD group ( $\mathrm{p}=0.021$, Tukey's test; Fig. 3E). 
A B $\quad$ C

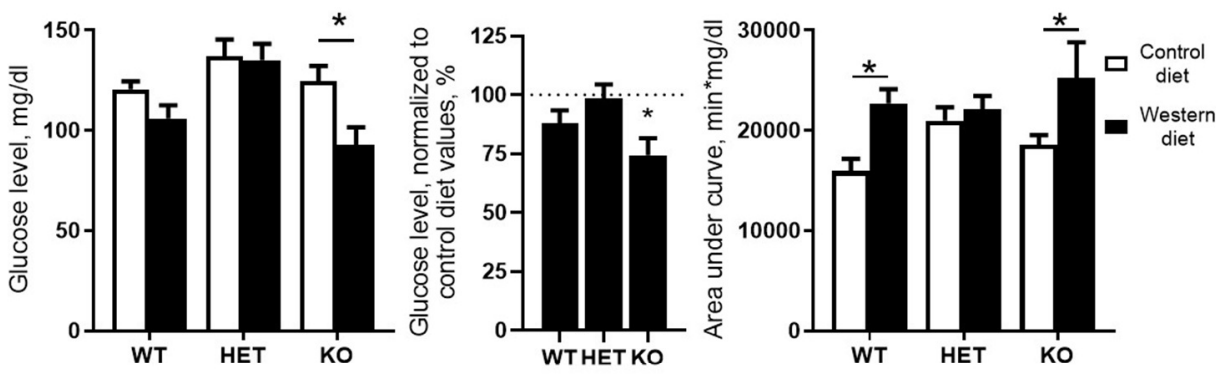

D

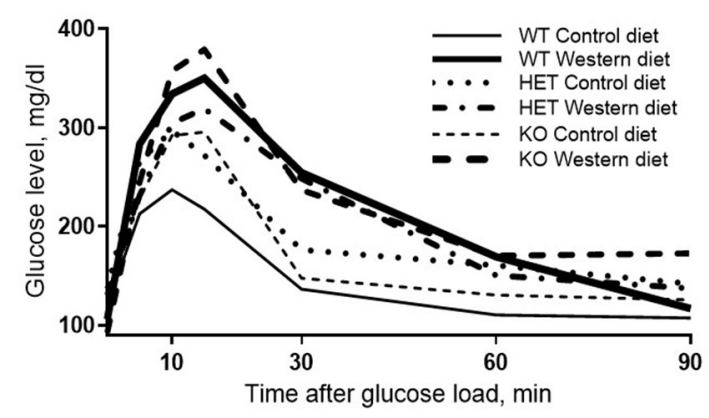

$\mathbf{E}$

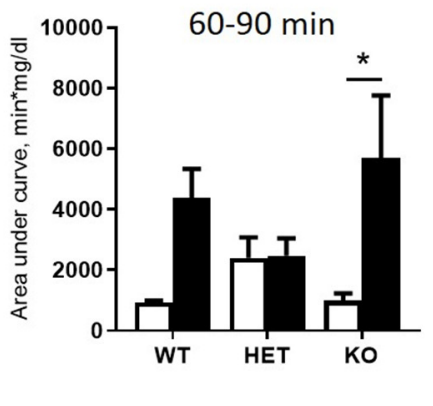

Figure 3. Effects of Western diet feeding on glucose tolerance. (A) Fasting blood glucose level after 18 hours of food deprivation. WD decreased fasting glucose level in KO mice (B) WD group basal glucose levels normalized to respective genotype group fed with $\mathrm{CD}$ values in comparison of with $100 \%$. (C) Area under glucose tolerance curve. WD increased area under the curve in WT and KO mice. (D) Glucose tolerance curve. (E) Area under glucose tolerance curve for the period between 60 and $90 \mathrm{~min} .{ }^{*} \mathrm{p}<0.05$, two-way ANOVA and Tukey's test (A,C,E) or one sample t test vs. $100 \%$ (B), 6-7 animals per group were used. Data are shown as mean $\pm \operatorname{SEM}(\mathrm{A}-\mathrm{C}, \mathrm{E})$ or mean $(\mathrm{D})$.

These results indicate that the impairment in glucose tolerance due to feeding with WD was exacerbated in $\mathrm{KO}$ mice in comparison to WT but was absent in HET group. 


\subsection{Effects of Western diet and SERT deficiency on the expression of markers of mitochondrial activity and Tlr4 in brain and liver}

A significant interaction between the genotype and diet was shown in mRNA levels of Ppargc1a in the hypothalamus and the hippocampus (two-way ANOVA, p $<0.05$, Table 2; Supplementary Fig. S2). The effect of genotype was significant in the dorsal raphe region and the prefrontal cortex $(\mathrm{p}<0.05$, Table 2). Post-hoc analysis revealed a significant increase of Ppargc1a expression levels in the hypothalamus and the prefrontal cortex of KO-CD in comparison to WT and HET mice fed with CD $(\mathrm{p}=0.039$ and $\mathrm{p}=0.045$ for hypothalamus and $\mathrm{p}=0.002$ and $\mathrm{p}=0.006$ for prefrontal cortex, Tukey's test; Fig. 4A). Ppargc1a expression in the hypothalamus of KO-WD was decreased in comparison to KO-CD ( $\mathrm{p}=0.045$, Tukey's test).

Two-way ANOVA revealed a significant genotype effect on mRNA levels of Ppargc1b in the hypothalamus ( $<<0.05$, Table 2). In addition, there was a significant diet effect in the dorsal raphe region and the prefrontal cortex $(\mathrm{p}<0.05$, Table 2$)$ on the expression of Ppargc1b. No differences were found in the hippocampus (Supplementary Fig. S2). Ppargc1b expression levels were decreased in the hypothalamus in KO and HET mice when compared to WT mice $(\mathrm{p}=0.0376$ and $\mathrm{p}=0.0095$, respectively, Tukey's test; Fig. 4B). 
Table 2. Two-way ANOVA results for statistical analysis of Ppargc1a and $b$ and Tlr4 gene expression.

\begin{tabular}{|c|c|c|c|c|c|c|c|}
\hline \multicolumn{8}{|c|}{ Brain gene expression } \\
\hline Gene & $\begin{array}{l}\text { Brain } \\
\text { struc- } \\
\text { ture }\end{array}$ & $\begin{array}{l}\text { Interac- } \\
\text { tion, } F\end{array}$ & $\begin{array}{l}\text { Interac- } \\
\text { tion, } p\end{array}$ & $\begin{array}{l}\text { Genotype, } \\
F\end{array}$ & $\begin{array}{l}\text { Genotype, } \\
p\end{array}$ & $\begin{array}{l}\text { Diet, } \\
F\end{array}$ & $\begin{array}{l}\text { Diet, } \\
p\end{array}$ \\
\hline \multirow[t]{4}{*}{ Ppargc1a } & HT & 6.003 & 0.0054 & 1.799 & 0.1793 & 0.1123 & 0.7394 \\
\hline & $\mathrm{DR}$ & 0.3308 & 0.7205 & 7.713 & 0.0017 & 3.750 & 0.0609 \\
\hline & HIP & 3.455 & 0.0418 & 1.745 & 0.1883 & 2.065 & 0.1589 \\
\hline & $\mathrm{PF}$ & 2.001 & 0.1492 & 10.46 & 0.0002 & 2.956 & 0.0937 \\
\hline \multirow[t]{4}{*}{ Ppargc1b } & HT & 0.2827 & 0.7554 & 5.436 & 0.0085 & 0.0061 & 0.9380 \\
\hline & DR & 0.1146 & 0.8921 & 0.9017 & 0.4166 & 5.301 & 0.0284 \\
\hline & HIP & 0.1623 & 0.8508 & 2.629 & 0.0856 & 0.0159 & 0.9001 \\
\hline & $\mathrm{PF}$ & 0.3177 & 0.7301 & 0.03676 & 0.9639 & 5.088 & 0.0313 \\
\hline \multirow[t]{4}{*}{ Tlr4 } & HT & 0.01194 & 0.9881 & 13.47 & $<0.0001$ & 0.0408 & 0.8409 \\
\hline & DR & 2.007 & 0.1509 & 4.994 & 0.0130 & 11.08 & 0.0022 \\
\hline & HIP & 0.6018 & 0.5529 & 1.317 & 0.2800 & 0.2996 & 0.5874 \\
\hline & $\mathrm{PF}$ & 1.370 & 0.2678 & 5.296 & 0.0100 & 2.930 & 0.0961 \\
\hline \multicolumn{8}{|c|}{ Liver gene expression } \\
\hline Gene & & $\begin{array}{l}\text { Interac- } \\
\text { tion, } F\end{array}$ & $\begin{array}{l}\text { Interac- } \\
\text { tion, } p\end{array}$ & $\begin{array}{l}\text { Genotype } \\
, F\end{array}$ & $\begin{array}{l}\text { Genotype } \\
, p\end{array}$ & $\begin{array}{l}\text { Diet, } \\
F\end{array}$ & $\begin{array}{l}\text { Diet, } \\
p\end{array}$ \\
\hline Ppargcla & & 6.052 & 0.0056 & 6.143 & 0.0053 & 1.733 & 0.1969 \\
\hline Ppargc1b & & 9.094 & 0.0007 & 10.46 & 0.0003 & 4.501 & 0.0412 \\
\hline Tlr4 & & 1.696 & 0.1978 & 0.2381 & 0.7893 & 1.668 & 0.2048 \\
\hline
\end{tabular}

$\mathrm{F}$ and $\mathrm{p}$ values are shown for interaction between genotype and diet, for genotype effect and for diet effect. HT - hypothalamus, DR - dorsal raphe region, HIP - hippocampus, PF - prefrontal cortex.

We found a significant effect of genotype on mRNA levels of Tlr4 in the hypothalamus and the prefrontal cortex (two-way ANOVA, 
$\mathrm{p}<0.05$, Table 2). Also, there was a significant diet effect in the dorsal raphe region $(\mathrm{p}<0.05$, Table 2$)$. No differences were found in the hippocampus (Supplementary Fig. S2). Post-hoc analysis showed an increase of Tlr4 expression levels in the hypothalamus of $\mathrm{KO}$ mice compared to WT and HET groups $(\mathrm{p}<0.001$ and $\mathrm{p}=0.005$, respectively, Tukey's test; Fig. 4C). In addition, a significant increase in Tlr4 expression was detected in the dorsal raphe region in $\mathrm{WT}$ and $\mathrm{KO}$ mice fed with WD compared to groups fed with CD $(\mathrm{p}=0.042$ and $\mathrm{p}=0.040$, respectively, Tukey's test) but not in HET mice, and in the prefrontal cortex in KO-WD group compared to WT-WD group $(\mathrm{p}=0.0403$, Tukey's test). Thus, the most prominent increase in Tlr4 expression was found in KO fed WD. No effect of WD on Tlr4 expression was observed in HET mice.

In the liver, there was a significant interaction between genotype and diet on the expression of Ppargc1a and Ppargc1b (two-way ANOVA, $\mathrm{p}<0.05$, Table 2). Ppargc1a and Ppargc1b expression levels in liver were decreased in WT-WD compared to WT-CD $(\mathrm{p}=0.026$ and $\mathrm{p}=0.002$, respectively, Tukey's test; Fig. 4D), and in HET and KO mice fed with CD compared to WT mice fed with CD ( $p=0.011$, $\mathrm{p}=0.002$ and $\mathrm{p}<0.001, \mathrm{p}<0.001$, respectively, Tukey's test). No differences between the groups were found in Tlr4 expression in the liver. 
Hypothalamus

A Ppargc1a
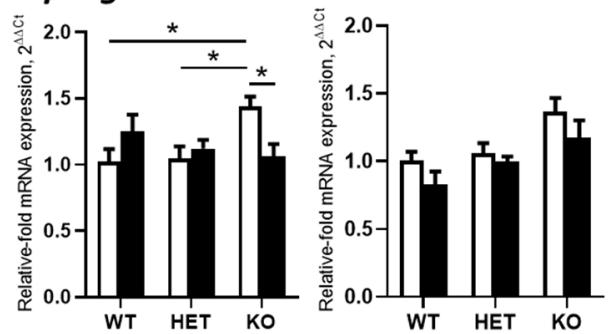

B Ppargc1b
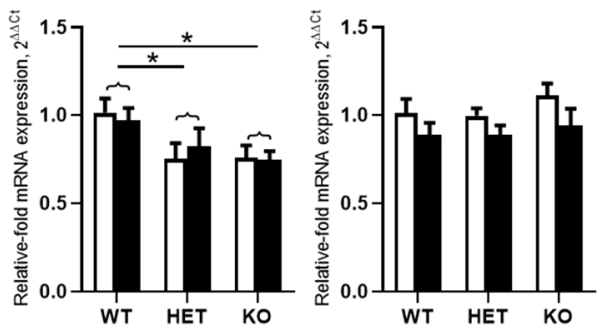

C TIr4
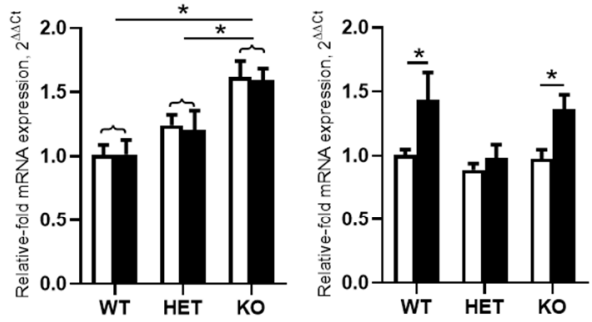

D Liver
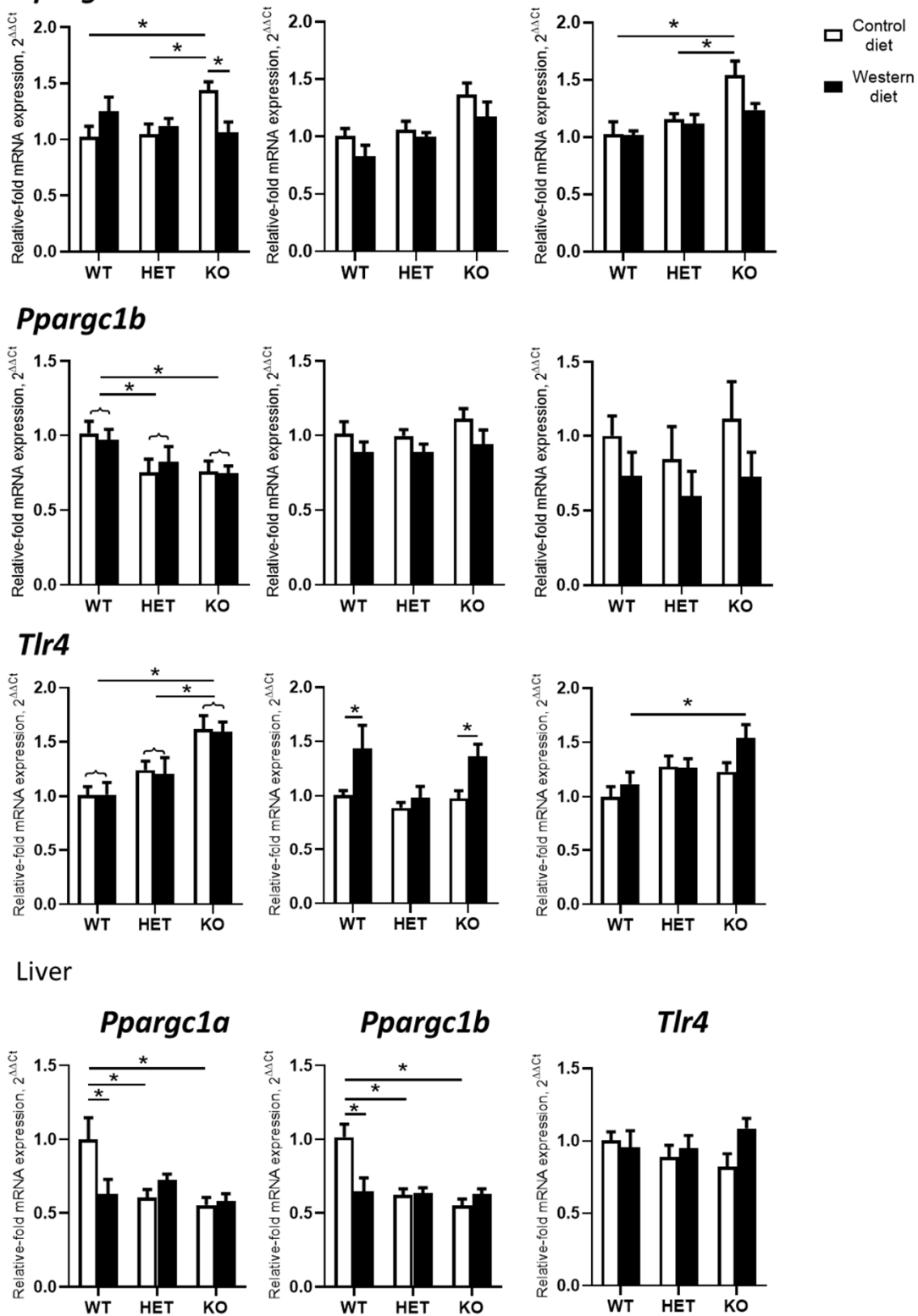

TIr4

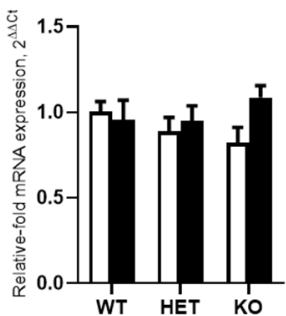

Figure 4. Effects of Western diet and SERT deficiency on brain and liver gene expression of markers of mitochondrial activity and Tlr4. (A-C) Ppargc1a, Ppargc1b and Tlr4 expression in hypothalamus, dorsal raphe 
region and prefrontal cortex. Tlr4 expression in dorsal raphe region was significantly increased in WT and KO but not HET mice fed with WD compared to respective groups fed with CD. (D) Ppargc1a, Ppargc1b and Tlr4 expression in the liver. ${ }^{*} \mathrm{p}<0.05$, two-way ANOVA and Tukey's test, $\}$ - main genotype effect, 6-7 animals per group were used. Data are shown as mean $\pm \mathrm{SEM}$.

\subsection{Changes in brain expression of serotonin receptors by Western diet and SERT deficiency}

Two-way ANOVA analysis demonstrated a significant genotype effect on the Htr1a mRNA levels in the dorsal raphe region and the prefrontal cortex $(\mathrm{p}<0.05$, Table 3). In addition, a significant effect of diet was shown in prefrontal cortex $(\mathrm{p}<0.05$, Table 3). Htr1a expression levels in the dorsal raphe region were decreased in HET and KO mice compared to WT mice $(\mathrm{p}<0.001$ and $\mathrm{p}=0.011$, respectively, Tukey's test; Fig. 5A).

There was a significant interaction between genotype and diet in mRNA levels of Htr2a in the hippocampus (two-way ANOVA, p $<0.05$, Table 3). Htr2a expression was elevated in the hippocampus of KO-CD mice compared to WT-CD and HET-CD mice $(\mathrm{p}=0.001$ and $\mathrm{p}=0.006$, Tukey's test; Fig. 5B).

A significant interaction between genotype and diet in mRNA levels of Htr1b receptor was found in the hypothalamus, the dorsal raphe region and the prefrontal cortex (two-way ANOVA, p $<0.05$, Table 3) but not in hippocampus. Post-hoc analysis revealed a significant increase in Htr1b expression in the hypothalamus, the dorsal raphe region and the prefrontal cortex of KO-CD group compared to 
WT-CD and HET-CD groups $(\mathrm{p}=0.040$ and $\mathrm{p}=0.020$ for hypothalamus, $\mathrm{p}<0.001$ and $\mathrm{p}<0.001$ for the dorsal raphe region, $\mathrm{p}=0.001$ and $\mathrm{p}=0.001$ for prefrontal cortex, Tukey's test; Fig. 5C).

Table 3. Two-way ANOVA results for statistical analysis of serotonin receptors gene expression.

\begin{tabular}{|c|c|c|c|c|c|c|c|}
\hline \multicolumn{8}{|c|}{ Brain gene expression } \\
\hline Gene & $\begin{array}{l}\text { Brain } \\
\text { struc- } \\
\text { ture }\end{array}$ & $\begin{array}{l}\text { Interac- } \\
\text { tion, } F\end{array}$ & $\begin{array}{l}\text { Interac- } \\
\text { tion, } p\end{array}$ & $\begin{array}{l}\text { Genotype, } \\
F\end{array}$ & $\begin{array}{l}\text { Genotype, } \\
p\end{array}$ & Diet, $F$ & Diet, $p$ \\
\hline \multirow[t]{4}{*}{ Htr1a } & $\mathrm{HT}$ & 0.07188 & 0.9308 & 2.915 & 0.0664 & 1.419 & 0.2409 \\
\hline & DR & 0.3828 & 0.6850 & 8.898 & 0.0008 & 0.00130 & 0.9715 \\
\hline & HIP & 0.5541 & 0.5792 & 2.066 & 0.1407 & 0.02632 & 0.8720 \\
\hline & $\mathrm{PF}$ & 0.8123 & 0.4523 & 6.690 & 0.0035 & 9.665 & 0.0038 \\
\hline \multirow[t]{4}{*}{ Htr2a } & HT & 0.4899 & 0.6165 & 0.8927 & 0.4180 & 0.8340 & 0.3669 \\
\hline & DR & 0.5235 & 0.5971 & 2.932 & 0.0669 & 0.2083 & 0.6510 \\
\hline & HIP & 3.485 & 0.0408 & 9.263 & 0.0005 & 1.188 & 0.2827 \\
\hline & $\mathrm{PF}$ & 0.3957 & 0.6759 & 2.289 & 0.1152 & 0.2406 & 0.6266 \\
\hline \multirow[t]{4}{*}{ Htr1b } & $\mathrm{HT}$ & 14.07 & $\begin{array}{l}<0.000 \\
1\end{array}$ & 1.108 & 0.3407 & 0.7577 & 0.3895 \\
\hline & DR & 8.429 & 0.0010 & 13.71 & $<0.0001$ & 2.331 & 0.1358 \\
\hline & HIP & 0.7293 & 0.4889 & 0.9170 & 0.4084 & 0.4561 & 0.5035 \\
\hline & $\mathrm{PF}$ & 4.426 & 0.0189 & 10.70 & 0.0002 & 1.309 & 0.2600 \\
\hline \multirow[t]{4}{*}{ Htr2c } & HT & 9.803 & 0.0004 & 10.14 & 0.0003 & 0.6533 & 0.4240 \\
\hline & DR & 5.308 & 0.0097 & 10.55 & 0.0003 & 6.429 & 0.0158 \\
\hline & HIP & 0.2895 & 0.7503 & 1.057 & 0.3574 & 0.6697 & 0.4183 \\
\hline & $\mathrm{PF}$ & 6.560 & 0.0036 & 6.111 & 0.0050 & 0.4158 & 0.5229 \\
\hline \multirow[t]{4}{*}{ Htr6 } & HT & 0.2326 & 0.7936 & 0.3814 & 0.6856 & 0.07229 & 0.7895 \\
\hline & DR & 0.05340 & 0.9481 & 5.580 & 0.0079 & 0.4079 & 0.5272 \\
\hline & HIP & 0.2349 & 0.7918 & 4.290 & 0.0211 & 0.3645 & 0.5497 \\
\hline & $\mathrm{PF}$ & 1.420 & 0.2546 & 0.2677 & 0.7666 & 0.09352 & 0.7615 \\
\hline
\end{tabular}

$\mathrm{F}$ and $\mathrm{p}$ values are shown for interaction between genotype and diet, for genotype effect and for diet effect. HT - hypothalamus, DR - dorsal raphe region, HIP - hippocampus, PF - prefrontal cortex. 
In addition, Htr1b expression levels were decreased in the hypothalamus and the dorsal raphe region of $\mathrm{KO}$ mice fed with WD in comparison to $\mathrm{KO}$ mice fed with $\mathrm{CD}$ ( $\mathrm{p}=0.006$ for hypothalamus, $\mathrm{p}=0.002$ for the dorsal raphe region, Tukey's test).

We found a significant interaction between genotype and diet in mRNA levels of Htr2c receptor in the hypothalamus, the dorsal raphe region and the prefrontal cortex (two-way ANOVA, p $<0.05$, Table 3), but not in hippocampus. Htr2c expression levels in the hypothalamus, the dorsal raphe region and the prefrontal cortex of $\mathrm{KO}$ mice fed with CD were increased compared to WT and HET mice fed with CD $(\mathrm{p}<0.001$ and $\mathrm{p}<0.001$ for hypothalamus, $\mathrm{p}=0.003$ and $\mathrm{p}<0.001$ for the dorsal raphe region, $\mathrm{p}=0.003$ and $\mathrm{p}=0.002$ for prefrontal cortex, Tukey's test; Fig. 5D). Decreased Htr2c expression levels in the hypothalamus and the dorsal raphe region of KO-WD compared to KO-CD were also detected $(\mathrm{p}=0.002$ for hypothalamus, $\mathrm{p}=0.003$ for the dorsal raphe region, Tukey's test).

There was a significant effect of genotype on mRNA levels of Htr6 receptor in the hippocampus and the dorsal raphe region $(\mathrm{p}<0.05$, Table 3). In hippocampus, Htr6 expression was increased in HET mice compared to WT ( $\mathrm{p}=0.029$, Tukey's test; Fig. 5E). Htr6 expression levels were significantly decreased in the dorsal raphe region of HET and KO mice compared to WT mice ( $\mathrm{p}=0.010$ and $\mathrm{p}=0.023$, Tukey's test). 
Hypothalamus

\section{A Htr1a}

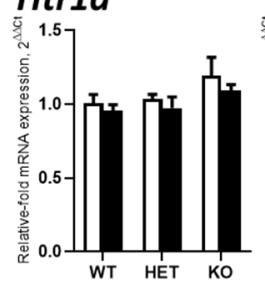

B Htr2a

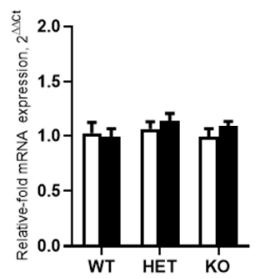

C Htr1b

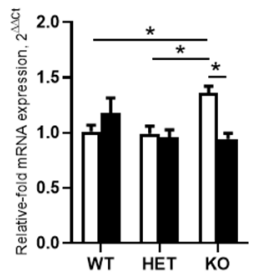

D Htr2c
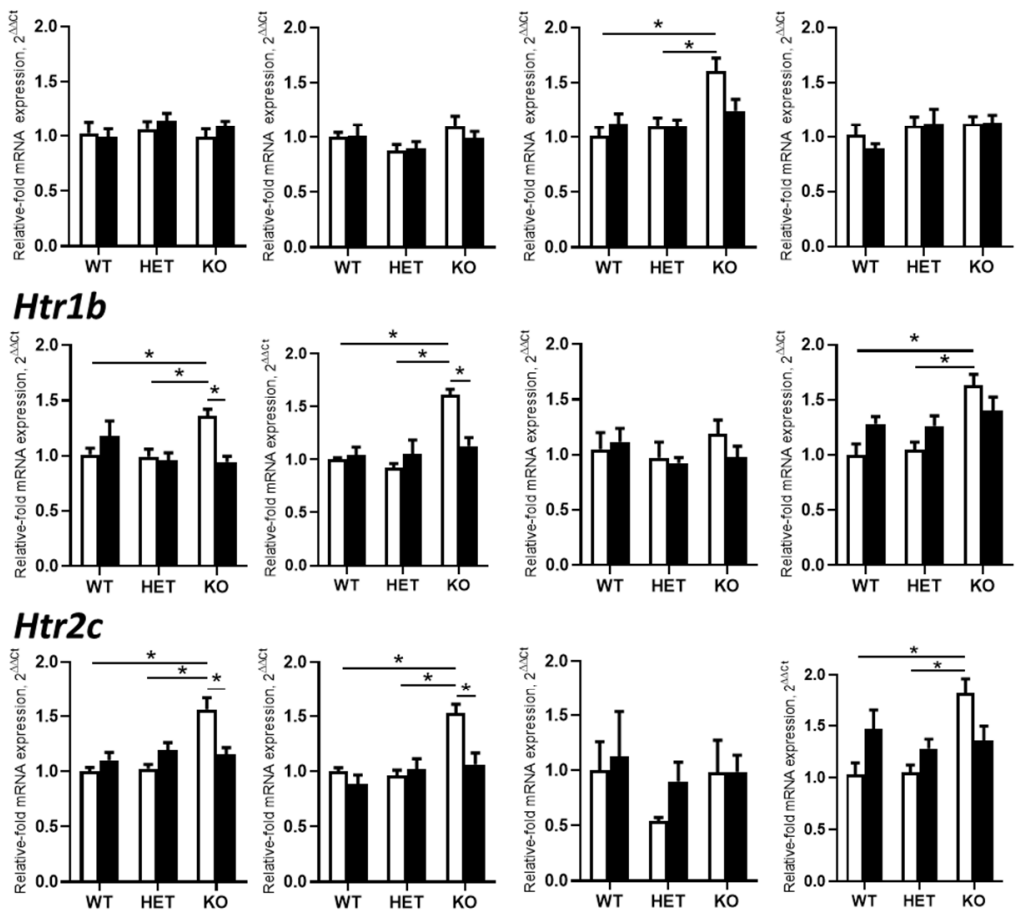

E Htr6


Hippocampus
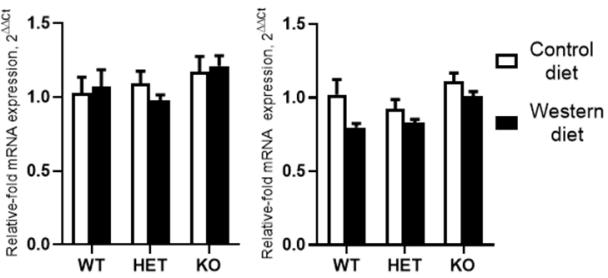

Prefrontal

cortex
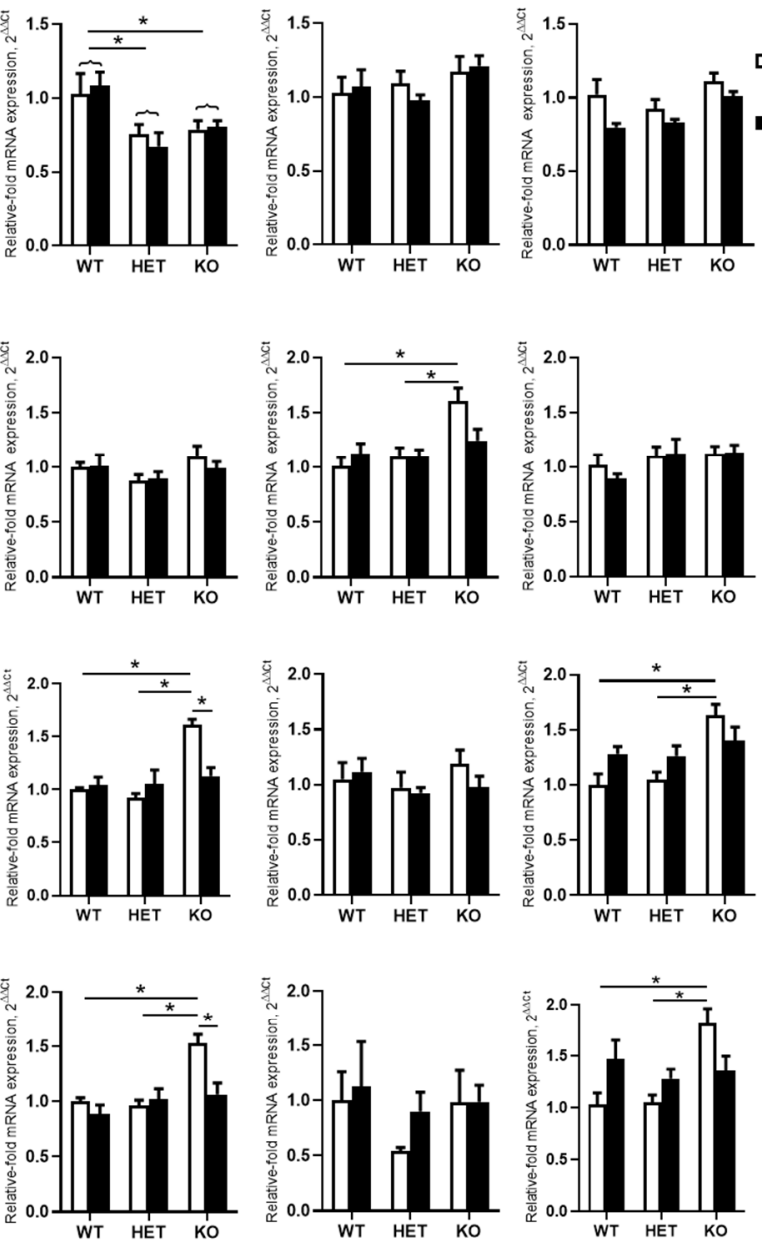

Figure 5. Changes in brain expression of serotonin receptors by Western diet and SERT deficiency. (A-B) Htr1a and Htr2a expression in hypothalamus, dorsal raphe region, hippocampus and prefrontal cortex. 
(C-D) Htr1b and Htr2c expression in the brain. KO mice fed with CD compared to WT and HET mice fed with CD displayed significant increase of Htr1b and Htr2c expression levels in hypothalamus, dorsal raphe region and prefrontal cortex. Compared to $\mathrm{KO}$ mice fed with $\mathrm{CD}, \mathrm{KO}$ mice fed with WD demonstrated significant decrease of Htr1b and Htr2c expression levels in hypothalamus and dorsal raphe region. (E) Htr6 expression in hypothalamus, dorsal raphe region, hippocampus and prefrontal cortex. ${ }^{*} \mathrm{p}<0.05$, two-way ANOVA and Tukey's test, \} - main genotype effect, 6-7 animals per group were used. Data are shown as mean \pm SEM.

That way, the expression of Htr1b, Htr2a, and Htr2c was increased in $\mathrm{KO}$ group fed with $\mathrm{CD}$. Feeding with WD inversed these changes. Sert-deficient mice, irrespective of the diet, demonstrated a decreased Htr1a and Htr6 expression in the dorsal raphe region.

\subsection{Western diet and SERT deficiency affected emotionality and hippocampus-dependent performance}

A comparison of exploratory rearing activity in the novel cage test during $1^{\text {st }}$ minute of the test by two-way ANOVA showed a significant genotype effect on the number of rears $(\mathrm{p}<0.05$, Table 4$)$. The number of rears was decreased in WD-fed KO group in comparison to WD-fed WT and HET mice $(\mathrm{p}=0.0034$ and $\mathrm{p}=0.0441$, respectively, Tukey's test; Fig. 6A), suggesting a reduced exploration in $\mathrm{KO}$ mice fed with WD.

In the O-maze test, there was a significant diet effect (two-way ANOVA, p $<0.05$, Table 4) on the latency to exit to open arm. This parameter was decreased in $\mathrm{KO}$ mice fed with WD compared to $\mathrm{KO}$ 
group fed with CD ( $\mathrm{p}=0.0095$, Tukey's test; Fig. 6B), which could be interpreted as a sign of increased impulsivity in KO-WD group.

Table 4. Two-way ANOVA results for statistical analysis of behavioural parameters.

\begin{tabular}{|l|l|l|l|l|l|l|}
\hline \multicolumn{7}{|l|}{ Behavioural parameters } \\
\hline Parameter & $\begin{array}{l}\text { Interaction, } \\
F\end{array}$ & $\begin{array}{l}\text { Interaction, } \\
p\end{array}$ & $\begin{array}{l}\text { Genotype, } \\
F\end{array}$ & $\begin{array}{l}\text { Genotype, } \\
p\end{array}$ & $\begin{array}{l}\text { Diet, } \\
F\end{array}$ & $\begin{array}{l}\text { Diet, } \\
p\end{array}$ \\
\hline $\begin{array}{l}\text { Number of } \\
\text { rears, } 1^{\text {st }} \text { min }\end{array}$ & 3.336 & 0.0502 & 5.034 & $\mathbf{0 . 0 1 3 6}$ & 1.680 & 0.2055 \\
\hline $\begin{array}{l}\text { Latency to exit } \\
\text { lit arm }\end{array}$ & 1.937 & 0.1652 & 1.888 & 0.1724 & 8.482 & $\mathbf{0 . 0 0 7 4}$ \\
\hline Latency to float & 1.080 & 0.3556 & 7.790 & $\mathbf{0 . 0 0 2 5}$ & 13.34 & $\mathbf{0 . 0 0 1 3}$ \\
\hline $\begin{array}{l}\text { Duration of } \\
\text { floating }\end{array}$ & 1.303 & 0.2903 & 3.514 & $\mathbf{0 . 0 4 5 9}$ & 33.47 & $<\mathbf{0 . 0 0 0 1}$ \\
\hline $\begin{array}{l}\text { Latency to } \\
\text { immobility }\end{array}$ & 0.08797 & 0.9161 & 1.601 & 0.2216 & 10.86 & $\mathbf{0 . 0 0 2 9}$ \\
\hline $\begin{array}{l}\text { Latency of } 50 \% \\
\text { accomplishment } \\
\text { in tube-test }\end{array}$ & 4.210 & $\mathbf{0 . 0 1 9 2}$ & 29.07 & $<\mathbf{0 . 0 0 0 1}$ & 27.17 & $<\mathbf{0 . 0 0 0 1}$ \\
\hline
\end{tabular}

$\mathrm{F}$ and $\mathrm{p}$ values are shown for interaction between genotype and diet, for genotype effect and for diet effect.

In the tail suspension test, two-way ANOVA revealed a significant diet effect (two-way ANOVA, $\mathrm{p}<0.05$, Table 4) on the latency to immobility (Fig. 6C). While no significant differences were observed between the groups, there was a trend of decreasing latency to immobility in groups fed with WD compared to the respective genotype-matched groups fed with CD. Significant genotype and diet effects $(\mathrm{p}<0.05$, Table 4) were found in the latency to floating in the forced swim test. Post-hoc analysis revealed a significant decrease in the latency to floating in HET mice fed WD and KO mice fed CD in comparison to CD-fed HET group $(\mathrm{p}=0.0276$ and $\mathrm{p}=0.0356$, 
respectively, Tukey's test, Fig. 6D). Total duration of floating was increased in WD-fed WT, HET, and KO groups compared to CD-fed mice (Supplementary Fig. S3). Correlation analysis revealed no significant correlation between mouse body weight and duration of floating, latency to floating, and latency to immobility $(\mathrm{p}>0.05)$. This data suggests that WD feeding induces depressive-like behaviour in all Sert genotypes.

In the pellet-displacement test, there was a significant interaction between the genotype and diet type $(\mathrm{p}<0.05$, Table 4$)$ in the latency of 50\%-performance accomplishment. The latency of 50\%-performance accomplishment was increased in WT-WD and KO-WD mice but not in HET-WD compared to mice fed with CD $(\mathrm{p}<0.001$ and $\mathrm{p}=0.037$, respectively, Tukey's test; Fig. 6E). In addition, this parameter was increased in KO group fed with CD compared to WT-CD and HET-CD $(\mathrm{p}<0.001$ and $\mathrm{p}=0.001$, respectively, Tukey's test $)$ as well as in $\mathrm{KO}$ group fed with WD compared to WT-WD and HET-WD $(\mathrm{p}<0.001$ and $\mathrm{p}<0.001$, respectively, Tukey's test). Results of this test suggest that complete Sert deficiency impairs hippocampal-dependent performance. The same effect was observed in WT and KO but not HET mice fed with WD. 
A

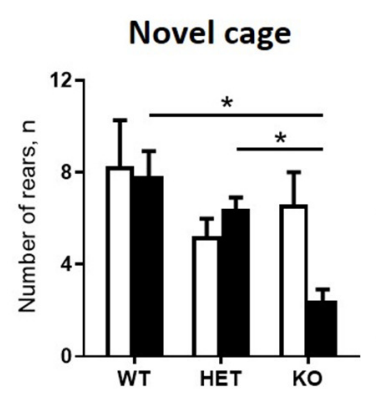

D

Forced swim test

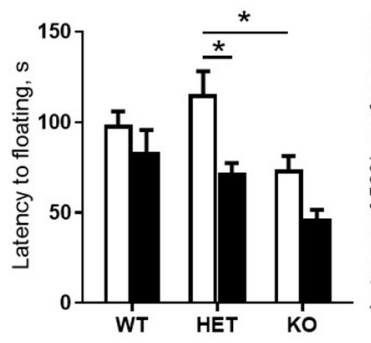

B

O-maze

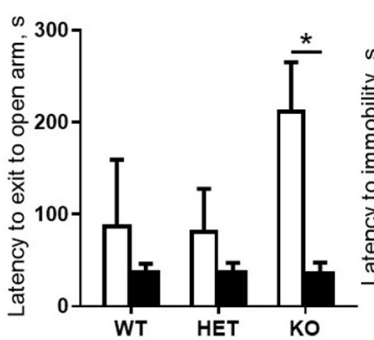

E

\section{Pellet-displacement}

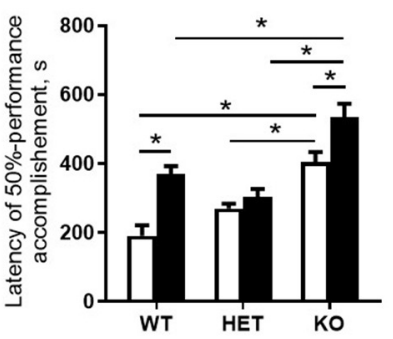

Figure 6. Western diet and SERT deficiency affected behavioural parameters, especially in KO mice. (A) Number of rears during the $1^{\text {st }}$ minute in novel cage test. KO group fed with WD compared to WT and HET mice fed with WD showed decrease in the number of rears. (B) Latency to exit to open arm in O-maze. The latency to exit to open arm was decreased in KO mice fed with WD compared to KO group fed with CD. (C) Latency to immobility in tail suspension test. (D) Latency to floating in forced swim test. Compared to HET mice fed with CD, the latency to floating was decreased in HET mice fed with WD and KO mice fed with CD. (E) Latency of 50\%-performance accomplishment in the pellet-displacement test. WT and KO mice fed with WD in comparison with mice fed with $\mathrm{CD}$ demonstrated increased latency of 50\%-performance accomplishment. This parameter was also significantly increased in $\mathrm{KO}$ mice fed with $\mathrm{CD}$ and $\mathrm{WD}$ compared to respective diet WT and HET groups. ${ }^{*} \mathrm{p}<0.05$, two-way ANOVA and Tukey's test, 6-7 animals per group were used. Data are shown as mean \pm SEM. 


\section{Discussion}

Our results have revealed that the effects of the WD are exacerbated in aged Sert KO mice on all our previously established metabolic, molecular and behavioural endpoints. Key hallmarks of the WD-induced syndrome were observed in the Sert-/- mice and WT controls, including decreased glucose tolerance, brain expression of Tlr4, disrupted hippocampus-dependent performance, but these were not observed in Sert+/- animals. However, all the genotypes challenged with WD displayed similar changes in weight gain, depressive-like behaviour, and suppressed expression of Ppargc1a and Ppargc1b in the liver. Most molecular changes that occurred in the two Sert-deficient genotypes developed regardless of diet. Overall, our data indicate distinct metabolic, molecular and behavioural effects of WD on aged mice with complete versus partial Sert inactivation and suggest resilience of Sert+/- mice to several key negative effects of WD. Effects of WD on mice with different Sert genotype and comparison of HET and KO groups fed CD with WT mice are summarized in Tables $\mathbf{5}$ and 6 . 
Table 5. Comparison of WD and CD groups with different Sert genotype.

\begin{tabular}{|c|c|c|c|}
\hline Parameter & $\begin{array}{l}\text { WT-WD } \\
\text { vs. } \\
\text { WT-CD }\end{array}$ & $\begin{array}{l}\text { HET-WD } \\
\text { vs. } \\
\text { HET-CD }\end{array}$ & $\begin{array}{l}\text { KO-WD } \\
\text { vs. } \\
\text { KO-CD }\end{array}$ \\
\hline \multicolumn{4}{|l|}{ Metabolism } \\
\hline Body weight & $\uparrow$ & $\uparrow$ & $\uparrow$ \\
\hline Food intake, week 1 & $\uparrow$ & $\uparrow$ & $\uparrow$ \\
\hline Water intake, weeks 2 and 3 & $=$ & $\downarrow$ & $\downarrow$ \\
\hline Fasting blood glucose & $=$ & $=$ & $\downarrow$ \\
\hline Glucose tolerance & $\downarrow$ & $=$ & $\downarrow \downarrow$ \\
\hline \multicolumn{4}{|l|}{ Liver gene expression } \\
\hline Ppargc $1 a$ and $b$ & $\downarrow$ & $=$ & $=$ \\
\hline \multicolumn{4}{|l|}{ Brain gene expression } \\
\hline Ppargc1a, HT & $=$ & $=$ & $\downarrow$ \\
\hline Tlr4, DR & $\uparrow \uparrow$ & $=$ & $\uparrow$ \\
\hline Htr1b, HT and DR & $=$ & $=$ & $\downarrow$ \\
\hline Htr2c, HT and DR & $=$ & $=$ & $\downarrow$ \\
\hline \multicolumn{4}{|l|}{ Behaviour } \\
\hline Rearing in novel cage & $=$ & $=$ & $\downarrow$ \\
\hline $\begin{array}{l}\text { Latency to exit open arm in O- } \\
\text { maze }\end{array}$ & $=$ & $=$ & $\downarrow$ \\
\hline Duration of floating in swim test & $\uparrow$ & $\uparrow$ & $\uparrow$ \\
\hline Latency to float in swim test & $=$ & $\downarrow$ & $=$ \\
\hline $\begin{array}{l}\text { Latency of } 50 \% \text {-performance } \\
\text { accomplishment in pellet } \\
\text { displacement test }\end{array}$ & $\uparrow$ & $=$ & $\uparrow \uparrow$ \\
\hline
\end{tabular}

Most of the metabolic, molecular and behavioural changes found in WT-WD mice were exacerbated in KO-WD. Some of the effects of the WD were absent in HET mice compared to WT and KO. HT - hypothalamus, DR - dorsal raphe region, HIP- hippocampus, PF - prefrontal cortex. Body weight was analyzed after 3 weeks of WD feeding. Food intake was measured in $\mathrm{kkal} / \mathrm{g}$ of body weight, water intake in $\mathrm{ml} / \mathrm{g}$ of body weight. 
Table 6. Comparison of HET and KO groups fed CD with WT mice.

\begin{tabular}{|c|c|c|}
\hline $\mathrm{P}_{\text {Parameter }}$ & $\begin{array}{c}\text { HET-CD } \\
\text { vs. } \\
\text { WT-CD }\end{array}$ & $\begin{array}{c}\text { KO-CD } \\
\text { vs. } \\
\text { WT-CD }\end{array}$ \\
\hline \multicolumn{3}{|l|}{ Metabolism } \\
\hline Basal body weight & $\uparrow$ & $\uparrow \uparrow$ \\
\hline Food intake, weeks 2 and 3 & $=$ & $\downarrow$ \\
\hline \multicolumn{3}{|l|}{ Liver gene expression } \\
\hline Ppargc $1 a$ and $b$ & $\downarrow$ & $\downarrow$ \\
\hline \multicolumn{3}{|l|}{ Brain gene expression } \\
\hline Ppargc1a, HT and PF & $=$ & $\uparrow$ \\
\hline Ppargc1b, HT & $\downarrow$ & $\downarrow$ \\
\hline $\mathrm{Tlr}_{4}, \mathrm{HT}$ & $=$ & $\uparrow$ \\
\hline Htr1a, DR & $\downarrow$ & $\downarrow$ \\
\hline Htr2a, HIP & $=$ & $\uparrow$ \\
\hline$H t r 1 b, \mathrm{HT}, \mathrm{DR}$ and PF & $=$ & $\uparrow$ \\
\hline Htr2c, HT, DR and PF & $=$ & $\uparrow$ \\
\hline Htr6, DR & $\downarrow$ & $\downarrow$ \\
\hline Htr6, HIP & $\uparrow$ & $=$ \\
\hline \multicolumn{3}{|l|}{ Behaviour } \\
\hline $\begin{array}{l}\text { Latency of } 50 \% \text {-performance } \\
\text { accomplishment in pellet displacement test }\end{array}$ & $=$ & $\uparrow$ \\
\hline
\end{tabular}

While some metabolic, molecular and behavioural differences with WT were similar in HET and KO, KO mice demonstrated a more distinguishable phenotype. HT - hypothalamus, DR - dorsal raphe region, HIP - hippocampus, PF - prefrontal cortex. Food intake was measured in $\mathrm{kkal} / \mathrm{g}$ of body weight.

The present study on aged mice has replicated the principal findings reported for young mice fed the WD, such as impaired glucose tolerance, altered expression of Tlr4, Ppargc1a and Ppargc1b (Strekalova et al., 2015, 2016) and signs of emotional and cognitive 
abnormalities (Strekalova et al., 2015, 2016; Veniaminova et al., 2016, $2017,2020)$. In comparison with young mice, aged animals exposed to WD gained weight and exhibited less profound changes in the expression of markers of inflammation and mitochondrial function. This is likely due to age-related alterations in the expression of these genes (Burgueño et al., 2013; Letiembre et al., 2007; Scarpulla, 2002). The aged mice displayed a prolonged increase of intake in the amount of calories after the switch to the highly caloric WD lasting, which lasted for at least one week instead of three days in young mice (Strekalova et al., 2015). This is in accordance with the well-described age-related dysregulation of consummatory behaviour and metabolic processes (Gill et al., 2015) in the WD model.

Sert/- mice fed a WD displayed greater changes in most dietinduced abnormalities that were found in the WT controls, including impairment of glucose tolerance, behavioural despair, suppressed novelty exploration and hippocampus-dependent performance, impulsivity in the O-maze and brain over-expression of Tlr4. At the end of the experiment, in comparison with other genotypes, Sert/- mice displayed a significant decrease in the intake of calories and lowered blood levels of fasting glucose after being fed the WD. Similar findings were obtained after a 24-week exposure of hamsters to a high-fat diet (Guo et al., 2016) and seemed to be underpinned by the facilitated conversion of blood glucose to lipids, in corollary to the present study, 
and Sert $^{/-}$mice revealed increased glucose absorption in a bowel (Greig et al., 2017). Previous work with WD-fed six-month-old male Sert/mice reported the opposite effects (Chen et al., 2012), but this appears to be due to a sex-related bias, and further supported by the dietary conditions used here (Comhair et al., 2011). Notably, the finding that there was reduced energy intake in Sert/- mutants rules out the possibility that their weight gain was due to increased diet intake. Instead, the metabolic changes described in Sert/- genotype are likely to be caused by functional disturbances in hypothalamic regulation of intake, which might be related to the over-expression of Tlr 4 that was observed in this brain structure. The activation of TLR4 by long-chain saturated fatty acids is considered as a major trigger of inflammatory mechanisms during excessive consumption of WD-like diets (Lancaster et al., 2018). Generalized brain over-expression of TLR4 on gene and protein levels was shown in WD-exposed young mice (Strekalova et al., 2015). Pro-inflammatory changes, and particularly, elevated expression of Tlr4 in the hypothalamus, a primary brain area regulating metabolism (Kahn and Flier, 2000) were shown to affect insulin receptor signaling (Benomar et al., 2013; Wellen and Hotamisligil, 2003; Zhao et al., 2017). Accumulating evidence indicates that neuroinflammatory processes of various cause markedly affects insulin receptor sensitivity (Olefsky and Glass, 2010; Savage et al., 2001). The dysregulation of insulin receptor-mediated signaling can result in a 
suppression of mitochondrial functions and decreased expression of Ppargca1 and PPargcb1 that is reported in the present study and in other experiments (Burgueño et al., 2013; Savage et al., 2001; Scarpulla, 2002; Strekalova et al., 2016). The latter results in impaired glucose tolerance, decreased metabolic rate and obesity (Kahn and Flier, 2000; Wellen and Hotamisligil, 2003). A close functional relationship between decreased SERT functions, pro-inflammatory changes and insulin resistance is well supported by the literature (Haub et al., 2010; Pomytkin et al., 2015, 2018).

Naïve Sert/- mice displayed increased brain expression of Ppargc1a, Htr2a, Htr1b and Htr2c that were not found in other genotypes and were "reversed" in WD-fed animals. Given the previously demonstrated association between most of these receptors with obesity and aging (Lee et al., 1998; Meltzer et al., 1998; Nonogaki et al., 2006; Ridderstråle et al., 2006; Simansky and Nicklous, 2002) it can be hypothesized that these changes may be adaptive, and WD disrupts these compensatory changes resulting in aggravation of metabolic and behavioural abnormalities in Sert/- mutants.

Expression of several genes was found to be similarly altered in both $\mathrm{Sert}^{/-}$and $\mathrm{Sert}^{+/}$genotypes, regardless dietary conditions. Decreases in expression of Ppargc1b, Htr1a, Htr6 in the brain, and of Ppargc1a and Ppargc1b in the liver were similar in both genotypes and were not affected by WD, suggesting ceiling effects of complete or 
partial Sert deficiency on these receptors. Earlier studies revealed lowered Htr1a receptor expression in the dorsal raphe region of female Sert/- and Sert ${ }^{+/-}$mice that was not found in the hypothalamus or hippocampus (Li et al., 2000). Altered function of 5-HT1A and 5-HT6 are known to underlie depressive-like behaviours (Savitz et al., 2009; Wesołowska, 2010), cognitive (Mitchell and Neumaier, 2005; Ögren et al., 2008) and social (Meneses, 2001; Wang et al., 2013) abnormalities, and likely to be implicated in the behavioural changes observed here.

The greater reduction of brain Ppargc1b expression in mutants than in controls could be associated with more pronounced obesity in the former groups. Similar to the changes in expression of serotonin receptors induced by WD reported here have been previously reported. For example, cafeteria and high-energy diets decreased brain expression of Htr2c receptor in rat (Beilharz et al., 2018; Lopez-Esparza et al., 2015). Mice exposed to high-fat diet displayed changes in expression of Htr2a in the olfactory nucleus and of Htr2c in the medial amygdaloid nucleus (Huang et al., 2004). 5-HT2C and 5-HT1B receptors were shown to play an inhibitory role in the regulation of calories intake, while elevated gene expression in the hypothalamus was proposed as a compensatory mechanism of hyperphagia in Ay mice (Nonogaki et al., 2006). 5-HT1B receptor was found to regulate food intake and insulin receptor sensitivity in mice with genetic inactivation of 5-HT2C receptor (Lee et al., 1998; Simansky and Nicklous, 2002). 
Similar to our results, compromised expression of 5-HT2C receptor was associated with obesity, abnormal feeding behaviour (Heisler et al., 1998) as well as insulin resistance and elevated blood glucose concentrations (Zhou et al., 2007). Finally, 5-HT6 receptor ligands have been demonstrated to improve insulin receptor sensitivity and regulate blood insulin, glucose concentrations, mechanisms of satiety and body weight (Heal et al., 2008).

Remarkably, unlike WT controls and Sert/-, mice Sert ${ }^{+/-}$mice did not reveal critical hallmarks of the WD-induced syndrome, such as decreases in glucose tolerance, brain expression of Tlr4 and disrupted hippocampus-dependent performance. In contrast to Sert/- they showed no changes in the brain expression of Ppargc1a, Htr2a, Htr1b and Htr1c receptors, decreases in calories and water intake, fasting glucose concentration, as well as novelty hypoexploration and impulsive-like behaviour in the elevated O-maze. Thus, Sert ${ }^{+/-}$mice were different from the Sert/- genotype consequences following WD challenge and exhibited partial resilience to its negative effects on the metabolic parameters and associated changes. This is in line with previous findings suggesting distinct physiology of two genotypes as has been shown for the expression of HPA regulatory protein binding protein 5 (FK506) in the pituitary of mice exposed to early life stress model (van der Doelen et al., 2014) and reduced basal corticosterone 
plasma levels and improved memory performance in the object recognition test in Sert ${ }^{+/-}$mice (van den Hove et al., 2011).

Relative resilience of $\mathrm{Sert}^{+/-}$mice to the diet might be viewed as an improved ability to adjust to environmental changes associated with this genotype (Belsky et al., 2009). It is also observed in clinical studies that showed beneficial effects of heterozygosity of SERT, such as higher cognitive function in elderly adults (Fiedorowicz et al., 2007) and other differences (Malmberg et al., 2008; van Dyck et al., 2005) and generally greater fitness in heterozygotes, because they show a broader range of gene expression than both homozygotes (Comings and MacMurray, 2000; Homberg and Lesch, 2011). This phenomenon is also discussed in framework of heterosis (Sonuga-Barke et al., 2011), or outbreeding enhancement, i.e. the improved or increased function of any biological quality in a hybrid offspring (Shull, 1948).

\section{Conclusions}

The comparison of the effects of the WD in Sert/- mice Sert ${ }^{+/-}$ reveals an intricate interplay between SERT deficiency and regulation of metabolism during aging. Thus, complete versus partial genetic SERT deficiency in aged mice is associated with distinct metabolic, molecular and behavioural consequences following the WD-challenge. While some diet-induced changes were similar in KO-WD and HET-WD mice, the latter displayed a "rescued" phenotype in terms of 
dietary-induced decrease in glucose tolerance, neuroinflammation and hippocampus-dependent behaviour. Sert deficiency was found to enhance inflammatory processes (Haub et al., 2010) and null-mutant Sert mice demonstrate higher susceptibility to the effects of WD on Tlr 4 expression.

\section{Acknowledgements}

The authors' work reported here was supported by the Deutsche Forschungsgemeinschaft (DFG: CRC TRR 58 A1/A5), the European Union's Seventh Framework Programme (FP7/2007-2013) under Grant No. 602805 (Aggressotype), ERA-Net NEURON/RESPOND, No. 01EW1602B and ERA-Net NEURON/DECODE, No. 01EW1902 (to KPL), the Horizon 2020 Research and Innovation Programme under Grant No. 728018 (Eat2beNICE) and Grant No. 643051 (MiND), 5-100 Russian Academic Excellence Project (to KPL and TS),

the Russian Science Foundation (RSF) project № 16-16-10073 (to IC and $\mathrm{OK}$ ) and the University of Würzburg in the funding programme Open Access Publishing (to KPL). We thank Collette Rousset and Donia Amrouni from C.Bernard University, Anna Gorlova from Sechenov University, Anastasiya Kibitkina, Galina Tolmacheva from Gorbatov Research Center for their valuable contribution to this work. 


\section{Author Contributions Statement}

KPL and TS conceived the study. TS, AS, OK, DA designed the experiments. EV, SM, TS, DA carried out the animal experiments. EV performed the molecular analyses. EV, RC, SM performed data analysis. IC, AK, DA, TS supervised the project. IC, OK, KPL, TS got the funding. EV, TS wrote the initial draft of the manuscript and all other authors (RC, IC, AS, SM, OK, AK, DA, KPL) revised it.

\section{Conflict of Interest Statement}

The authors declare that the research was conducted in the absence of any commercial or financial relationships that could be construed as a potential conflict of interest.

\section{References}

Batsis, J. A., and Zagaria, A. B. (2018). Addressing Obesity in Aging Patients. Med. Clin. North Am. 102, 65-85. doi:10.1016/j.mcna.2017.08.007.

Bearer, E. L., Barto, D., Reviere, A. R. H., and Jacobs, R. E. (2018). Neural activation imaged by MEMRI in mouse models of PTSD: Early Life Stress and Role of the Serotonergic System in Prolonged Response to Fear. Proc. Int. Soc. Magn. Reson. Med. Sci. Meet. Exhib. Int. Soc. Magn. Reson. Med. Sci. Meet. Exhib. 2018.

Available at: http://www.ncbi.nlm.nih.gov/pubmed/30349424.

Beilharz, J. E., Kaakoush, N. O., Maniam, J., and Morris, M. J. (2018). Cafeteria diet and probiotic therapy: cross talk among memory, neuroplasticity, serotonin receptors and gut microbiota in the rat. Mol. Psychiatry 23, 351-361. doi:10.1038/mp.2017.38.

Belsky, J., Jonassaint, C., Pluess, M., Stanton, M., Brummett, B., and Williams, R. (2009). Vulnerability genes or plasticity genes? Mol. Psychiatry 14, 746-754. doi:10.1038/mp.2009.44.

Benomar, Y., Gertler, A., De Lacy, P., Crépin, D., Ould Hamouda, H., Riffault, L., et al. (2013). Central Resistin Overexposure Induces Insulin 
Resistance Through Toll-Like Receptor 4. Diabetes 62, 102-114. doi:10.2337/db12-0237.

Bickerdike, M. J. (2003). 5-HT2C receptor agonists as potential drugs for the treatment of obesity. Curr. Top. Med. Chem. 3, 885-97. http://www.ncbi.nlm.nih.gov/pubmed/12678838.

Boemi, M., Furlan, G., and Luconi, M. P. (2016). "Glucose Metabolism, Insulin, and Aging," in Molecular Basis of Nutrition and Aging (Elsevier), 393-409. doi:10.1016/B978-0-12-801816-3.00029-7.

Burgueño, A. L., Cabrerizo, R., Gonzales Mansilla, N., Sookoian, S., and Pirola, C. J. (2013). Maternal high-fat intake during pregnancy programs metabolic-syndrome-related phenotypes through liver mitochondrial DNA copy number and transcriptional activity of liver PPARGC1A. J. Nutr. Biochem. 24, 6-13. doi:10.1016/j.jnutbio.2011.12.008.

Caspi, A., Hariri, A. R., Holmes, A., Uher, R., and Moffitt, T. E. (2010). Genetic sensitivity to the environment: the case of the serotonin transporter gene and its implications for studying complex diseases and traits. Am. J. Psychiatry 167, 509-27. doi:10.1176/appi.ajp.2010.09101452.

Chen, X., Margolis, K. J., Gershon, M. D., Schwartz, G. J., and Sze, J. Y. (2012). Reduced Serotonin Reuptake Transporter (SERT) Function Causes Insulin Resistance and Hepatic Steatosis Independent of Food Intake. PLoS One 7, e32511. doi:10.1371/journal.pone.0032511.

Collier, D. A., Stöber, G., Li, T., Heils, A., Catalano, M., Di Bella, D., et al. (1996). A novel functional polymorphism within the promoter of the serotonin transporter gene: possible role in susceptibility to affective disorders. Mol. Psychiatry 1, 453-60. Available at: http://www.ncbi.nlm.nih.gov/pubmed/9154246.

Comhair, T. M., Garcia Caraballo, S. C., Dejong, C. H., Lamers, W. H., and Koehler, S. E. (2011). Dietary cholesterol, female gender and n-3 fatty acid deficiency are more important factors in the development of nonalcoholic fatty liver disease than the saturation index of the fat. Nutr. Metab. (Lond). 8, 4. doi:10.1186/1743-7075-8-4.

Comings, D. E., and MacMurray, J. P. (2000). Molecular Heterosis: A Review. Mol. Genet. Metab. 71, 19-31. doi:10.1006/mgme.2000.3015.

Costa-Nunes, J. P., Cline, B. H., Araújo-Correia, M., Valença, A., Markova, N., Dolgov, O., et al. (2015). Animal Models of Depression and Drug Delivery with Food as an Effective Dosing Method: Evidences from Studies with Celecoxib and Dicholine Succinate. Biomed Res. Int. 2015, 596126. doi:10.1155/2015/596126. 
Couch, Y., Anthony, D. C., Dolgov, O., Revischin, A., Festoff, B., Santos, A. I., et al. (2013). Microglial activation, increased TNF and SERT expression in the prefrontal cortex define stress-altered behaviour in mice susceptible to anhedonia. Brain. Behav. Immun. 29, 136-146. doi:10.1016/j.bbi.2012.12.017.

Deacon, R. M. ., Croucher, A., and Rawlins, J. N. P. (2002). Hippocampal cytotoxic lesion effects on species-typical behaviours in mice. Behav. Brain Res. 132, 203-213. doi:10.1016/S0166-4328(01)00401-6.

Fiedorowicz, J. G., Moser, D. J., Hynes, S. M., Beglinger, L. J., Schultz, S. K., and Ellingrod, V. L. (2007). LA allelic heterozygosity of the 5HTTLPR polymorphism is associated with higher cognitive function and lower interpersonal sensitivity. Psychiatr. Genet. 17, 3-4. doi:10.1097/YPG.0b013e328010f498.

Fuemmeler, B. F., Agurs-Collins, T. D., Mcclernon, F. J., Kollins, S. H., Kail, M. E., Bergen, A. W., et al. (2008). Genes Implicated in Serotonergic and Dopaminergic Functioning Predict BMI Categories. Obesity 16, 348-355. doi:10.1038/oby.2007.65.

Giannaccini, G., Betti, L., Palego, L., Marsili, A., Santini, F., Pelosini, C., et al. (2013). The expression of platelet serotonin transporter (SERT) in human obesity. BMC Neurosci. 14, 128. doi:10.1186/1471-2202-14-128.

Gill, L. E., Bartels, S. J., and Batsis, J. A. (2015). Weight Management in Older Adults. Curr. Obes. Rep. 4, 379-88. doi:10.1007/s13679-015-0161-z.

Greenberg, B. D., Li, Q., Lucas, F. R., Hu, S., Sirota, L. A., Benjamin, J., et al. (2000). Association between the serotonin transporter promoter polymorphism and personality traits in a primarily female population sample. Am. J. Med. Genet. 96, 202-16. doi:10.1002/(sici)10968628(20000403)96:2<202::aid-ajmg16>3.0.co;2-j.

Greig, C. J., Zhang, L., and Cowles, R. A. (2017). Serotonin Reuptake Transporter Knockout Mice Exhibit Increased Enterocyte Mass and Intestinal Glucose Absorption. J. Am. Coll. Surg. 225, S157. doi:10.1016/j.jamcollsurg.2017.07.353.

Guo, W., Jiang, C., Yang, L., Li, T., Liu, X., Jin, M., et al. (2016). Quantitative Metabolomic Profiling of Plasma, Urine, and Liver Extracts by 1 H NMR Spectroscopy Characterizes Different Stages of Atherosclerosis in Hamsters. J. Proteome Res. 15, 3500-3510.

doi:10.1021/acs.jproteome.6b00179.

Haub, S., Ritze, Y., Bergheim, I., Pabst, O., Gershon, M. D., and Bischoff, S. C. (2010). Enhancement of intestinal inflammation in mice lacking 
interleukin 10 by deletion of the serotonin reuptake transporter. Neurogastroenterol. Motil. 22, 826-34, e229. doi:10.1111/j.13652982.2010.01479.x.

Heal, D. J., Smith, S. L., Fisas, A., Codony, X., and Buschmann, H. (2008). Selective 5-HT6 receptor ligands: progress in the development of a novel pharmacological approach to the treatment of obesity and related metabolic disorders. Pharmacol. Ther. 117, 207-31. doi:10.1016/j.pharmthera.2007.08.006.

Heisler, L. K., Chu, H.-M., and Tecott, L. H. (1998). Epilepsy and Obesity in Serotonin 5-HT2C Receptor Mutant Mice. Ann. N. Y. Acad. Sci. 861, 7478. doi:10.1111/j.1749-6632.1998.tb10175.x.

Herrera-Pérez, J. J., Fernández-Guasti, A., and Martínez-Mota, L. (2013). Brain SERT Expression of Male Rats Is Reduced by Aging and Increased by Testosterone Restitution. Neurosci. J. 2013, 1-8. doi:10.1155/2013/201909.

Homberg, J. R., la Fleur, S. E., and Cuppen, E. (2010). Serotonin Transporter Deficiency Increases Abdominal Fat in Female, but Not Male Rats. Obesity 18, 137-145. doi:10.1038/oby.2009.139.

Homberg, J. R., and Lesch, K.-P. (2011). Looking on the Bright Side of Serotonin Transporter Gene Variation. Biol. Psychiatry 69, 513-519. doi:10.1016/j.biopsych.2010.09.024.

Huang, X.-F., Han, M., and Storlien, L. H. (2004). Differential expression of 5-HT2A and 5-HT2C receptor mRNAs in mice prone, or resistant, to chronic high-fat diet-induced obesity. Mol. Brain Res. 127, 39-47. doi:10.1016/j.molbrainres.2004.05.008.

Iordanidou, M., Tavridou, A., Petridis, I., Arvanitidis, K. I., Christakidis, D., Vargemezis, V., et al. (2010). The serotonin transporter promoter polymorphism (5-HTTLPR) is associated with type 2 diabetes. Clin. Chim. Acta 411, 167-171. doi:10.1016/j.cca.2009.10.022.

Jiang, X., Wang, J., Luo, T., and Li, Q. (2009). Impaired hypothalamicpituitary-adrenal axis and its feedback regulation in serotonin transporter knockout mice. Psychoneuroendocrinology 34, 317-31. doi:10.1016/j.psyneuen.2008.09.011.

Kahn, B. B., and Flier, J. S. (2000). Obesity and insulin resistance. J. Clin. Invest. 106, 473-481. doi:10.1172/JCI10842.

Kakiuchi, T., Tsukada, H., Fukumoto, D., and Nishiyama, S. (2001). Effects of aging on serotonin transporter availability and its response to fluvoxamine in the living brain: PET study with $[11 \mathrm{C}](+) \mathrm{McN} 5652$ and $[11 \mathrm{C}]$ $(-)$ McN5652 in conscious monkeys. Synapse 40, 170-179. 
doi:10.1002/syn.1039.

Karakelides, H., Irving, B. A., Short, K. R., O'Brien, P., and Nair, K. S. (2010). Age, Obesity, and Sex Effects on Insulin Sensitivity and Skeletal Muscle Mitochondrial Function. Diabetes 59, 89-97. doi:10.2337/db09-0591.

Kautzky-Willer, A., Harreiter, J., and Pacini, G. (2016). Sex and Gender Differences in Risk, Pathophysiology and Complications of Type 2 Diabetes Mellitus. Endocr. Rev. 37, 278-316. doi:10.1210/er.2015-1137.

Khabazkhoob, M., Emamian, M. H., Hashemi, H., Shariati, M., and Fotouhi, A. (2017). Prevalence of Overweight and Obesity in the Middle-age Population: A Priority for the Health System. Iran. J. Public Health 46, 827834. Available at: http://www.ncbi.nlm.nih.gov/pubmed/28828326.

Koopman, K. E., Booij, J., Fliers, E., Serlie, M. J., and la Fleur, S. E. (2013). Diet-induced changes in the Lean Brain: Hypercaloric high-fat-highsugar snacking decreases serotonin transporters in the human hypothalamic region. Mol. Metab. 2, 417-22. doi:10.1016/j.molmet.2013.07.006.

Lancaster, G. I., Langley, K. G., Berglund, N. A., Kammoun, H. L., Reibe, S., Estevez, E., et al. (2018). Evidence that TLR4 Is Not a Receptor for Saturated Fatty Acids but Mediates Lipid-Induced Inflammation by Reprogramming Macrophage Metabolism. Cell Metab. 27, 1096-1110.e5. doi:10.1016/j.cmet.2018.03.014.

Lee, M. D., Aloyo, V. J., Fluharty, S. J., and Simansky, K. J. (1998). Infusion of the serotonin1B (5-HT1B) agonist CP-93,129 into the parabrachial nucleus potently and selectively reduces food intake in rats. Psychopharmacology (Berl). 136, 304-7.

Available at: http://www.ncbi.nlm.nih.gov/pubmed/9566817.

Lesch, K. P., Bengel, D., Heils, A., Sabol, S. Z., Greenberg, B. D., Petri, S., et al. (1996). Association of anxiety-related traits with a polymorphism in the serotonin transporter gene regulatory region. Science 274, 1527-31. Available at: http://www.ncbi.nlm.nih.gov/pubmed/8929413.

Letiembre, M., Hao, W., Liu, Y., Walter, S., Mihaljevic, I., Rivest, S., et al. (2007). Innate immune receptor expression in normal brain aging. Neuroscience 146, 248-54. doi:10.1016/j.neuroscience.2007.01.004.

Li, Q., Wichems, C., Heils, A., Lesch, K. P., and Murphy, D. L. (2000). Reduction in the density and expression, but not G-protein coupling, of serotonin receptors (5-HT1A) in 5-HT transporter knock-out mice: gender and brain region differences. J. Neurosci. 20, 7888-95. http://www.ncbi.nlm.nih.gov/pubmed/11050108.

Lopez-Esparza, S., Berumen, L. C., Padilla, K., Miledi, R., and García- 
Alcocer, G. (2015). Expression of hippocampal serotonin receptors 5-HT2C and 5-HT5A in a rat model of diet-induced obesity supplemented with tryptophan. Int. J. Dev. Neurosci. 42, 80-85.

doi:10.1016/j.ijdevneu.2015.02.010.

Malatynska, E., Steinbusch, H. W. M., Redkozubova, O., Bolkunov, A., Kubatiev, A., Yeritsyan, N. B., et al. (2012). Anhedonic-like traits and lack of affective deficits in 18-month-old C57BL/6 mice: Implications for modeling elderly depression. Exp. Gerontol. 47, 552-564. doi:10.1016/j.exger.2012.04.010.

Malmberg, K., Wargelius, H.-L., Lichtenstein, P., Oreland, L., and Larsson, J.-O. (2008). ADHD and Disruptive behavior scores - associations with MAO-A and 5-HTT genes and with platelet MAO-B activity in adolescents. BMC Psychiatry 8, 28. doi:10.1186/1471-244X-8-28.

Matuskey, D., Pittman, B., Planeta-Wilson, B., Walderhaug, E., Henry, S., Gallezot, J.-D., et al. (2012). Age Effects on Serotonin Receptor 1B as Assessed by PET. J. Nucl. Med. 53, 1411-1414.

doi:10.2967/jnumed.112.103598.

Meltzer, C. C., Smith, G., DeKosky, S. T., Pollock, B. G., Mathis, C. A., Moore, R. Y., et al. (1998). Serotonin in Aging, Late-Life Depression, and Alzheimer\&\#39;s Disease: The Emerging Role of Functional Imaging. Neuropsychopharmacology 18, 407.

Available at: https://doi.org/10.1016/S0893-133X(97)00194-2.

Meneses, A. (2001). Effects of the 5-HT6 receptor antagonist Ro 04-6790 on learning consolidation. Behav. Brain Res. 118, 107-110. doi:10.1016/S0166-4328(00)00316-8.

Millan, M. J. (2005). Serotonin 5-HT2C receptors as a target for the treatment of depressive and anxious states: focus on novel therapeutic strategies. Therapie 60, 441-60.

Available at: http://www.ncbi.nlm.nih.gov/pubmed/16433010.

Mitchell, E. S., and Neumaier, J. F. (2005). 5-HT6 receptors: a novel target for cognitive enhancement. Pharmacol. Ther. 108, 320-333. doi:10.1016/j.pharmthera.2005.05.001.

Morita, Y., Iwamoto, I., Mizuma, N., Kuwahata, T., Matsuo, T., Yoshinaga, M., et al. (2006). Precedence of the shift of body-fat distribution over the change in body composition after menopause. J. Obstet. Gynaecol. Res. 32, 513-6.

doi:10.1111/j.1447-0756.2006.00437.x.

Murphy, D. L., Lerner, A., Rudnick, G., and Lesch, K.-P. (2004). 
Serotonin transporter: gene, genetic disorders, and pharmacogenetics. Mol. Interv. 4, 109-23. doi:10.1124/mi.4.2.8.

Murphy, D. L., and Lesch, K.-P. (2008). Targeting the murine serotonin transporter: insights into human neurobiology. Nat. Rev. Neurosci. 9, 85-96. doi:10.1038/nrn2284.

Nam, S. B., Kim, K., Kim, B. S., Im, H.-J., Lee, S. H., Kim, S.-J., et al. (2018). The Effect of Obesity on the Availabilities of Dopamine and Serotonin Transporters. Sci. Rep. 8, 4924. doi:10.1038/s41598-018-22814-8.

Nonogaki, K., Nozue, K., and Oka, Y. (2006). Hyperphagia Alters Expression of Hypothalamic 5-HT2C and 5-HT1B Receptor Genes and Plasma Des-Acyl Ghrelin Levels in A y Mice. Endocrinology 147, 5893-5900. doi:10.1210/en.2006-0418.

Ögren, S. O., Eriksson, T. M., Elvander-Tottie, E., D'Addario, C., Ekström, J. C., Svenningsson, P., et al. (2008). The role of 5-HT1A receptors in learning and memory. Behav. Brain Res. 195, 54-77. doi:10.1016/j.bbr.2008.02.023.

Olefsky, J. M., and Glass, C. K. (2010). Macrophages, Inflammation, and Insulin Resistance. Annu. Rev. Physiol. 72, 219-246. doi:10.1146/annurevphysiol-021909-135846.

Pomytkin, I. A., Cline, B. H., Anthony, D. C., Steinbusch, H. W., Lesch, K.-P., and Strekalova, T. (2015). Endotoxaemia resulting from decreased serotonin tranporter (5-HTT) function: a reciprocal risk factor for depression and insulin resistance? Behav. Brain Res. 276, 111-7. doi:10.1016/j.bbr.2014.04.049.

Pomytkin, I., Costa-Nunes, J. P., Kasatkin, V., Veniaminova, E., Demchenko, A., Lyundup, A., et al. (2018). Insulin receptor in the brain: Mechanisms of activation and the role in the CNS pathology and treatment. CNS Neurosci. Ther. doi:10.1111/cns.12866.

Ridderstråle, M., Johansson, L. E., Rastam, L., and Lindblad, U. (2006). Increased risk of obesity associated with the variant allele of the PPARGC1A Gly482Ser polymorphism in physically inactive elderly men. Diabetologia 49, 496-500. doi:10.1007/s00125-005-0129-8.

Rodríguez, J. J., Noristani, H. N., and Verkhratsky, A. (2012). The serotonergic system in ageing and Alzheimer's disease. Prog. Neurobiol. 99, 15-41. doi:10.1016/j.pneurobio.2012.06.010.

Savage, D. B., Sewter, C. P., Klenk, E. S., Segal, D. G., Vidal-Puig, A., Considine, R. V., et al. (2001). Resistin / Fizz3 Expression in Relation to Obesity and Peroxisome Proliferator-Activated Receptor- Action in Humans. 
Diabetes 50, 2199-2202. doi:10.2337/diabetes.50.10.2199.

Savitz, J., Lucki, I., and Drevets, W. C. (2009). 5-HT(1A) receptor function in major depressive disorder. Prog. Neurobiol. 88, 17-31. doi:10.1016/j.pneurobio.2009.01.009.

Scarpulla, R. C. (2002). Nuclear activators and coactivators in mammalian mitochondrial biogenesis. Biochim. Biophys. Acta 1576, 1-14. doi:10.1016/s0167-4781(02)00343-3.

Shull, G. H. (1948). What Is "Heterosis"? Genetics 33, 439-46. Available at: http://www.ncbi.nlm.nih.gov/pubmed/17247290.

Simansky, K. J., and Nicklous, D. M. (2002). Parabrachial infusion of Dfenfluramine reduces food intake. Blockade by the 5-HT(1B) antagonist SB216641. Pharmacol. Biochem. Behav. 71, 681-90.

http://www.ncbi.nlm.nih.gov/pubmed/11888559.

Sonuga-Barke, E. J. S., Kumsta, R., Schlotz, W., Lasky-Su, J., Marco, R., Miranda, A., et al. (2011). A Functional Variant of the Serotonin Transporter Gene (SLC6A4) Moderates Impulsive Choice in AttentionDeficit/Hyperactivity Disorder Boys and Siblings. Biol. Psychiatry 70, 230 236. doi:10.1016/j.biopsych.2011.01.040.

Sookoian, S., Gemma, C., García, S. I., Fernández Gianotti, T., Dieuzeide, G., Roussos, A., et al. (2007). Short Allele of Serotonin Transporter Gene Promoter Is a Risk Factor for Obesity in Adolescents*. Obesity 15, 271-276. doi:10.1038/oby.2007.519.

Strekalova, T., Costa-Nunes, J. P., Veniaminova, E., Kubatiev, A., Lesch, K.-P., Chekhonin, V. P., et al. (2016). Insulin receptor sensitizer, dicholine succinate, prevents both Toll-like receptor 4 (TLR4) upregulation and affective changes induced by a high-cholesterol diet in mice. J. Affect. Disord. 196, 109-116. doi:10.1016/j.jad.2016.02.045.

Strekalova, T., Evans, M., Costa-Nunes, J., Bachurin, S., Yeritsyan, N., Couch, Y., et al. (2015). Tlr4 upregulation in the brain accompanies depression- and anxiety-like behaviors induced by a high-cholesterol diet. Brain. Behav. Immun. 48. doi:10.1016/j.bbi.2015.02.015.

Strekalova, T., and Steinbusch, H. W. M. (2010). Measuring behavior in mice with chronic stress depression paradigm. Prog. Neuropsychopharmacol. Biol. Psychiatry 34, 348-61. doi:10.1016/j.pnpbp.2009.12.014.

Stuart, M. J., and Baune, B. T. (2012). Depression and type 2 diabetes: inflammatory mechanisms of a psychoneuroendocrine co-morbidity. Neurosci. Biobehav. Rev. 36, 658-76. doi:10.1016/j.neubiorev.2011.10.001.

Üçeyler, N., Schütt, M., Palm, F., Vogel, C., Meier, M., Schmitt, A., et 
al. (2010). Lack of the serotonin transporter in mice reduces locomotor activity and leads to gender-dependent late onset obesity. Int. J. Obes. 34, 701. Available at: https://doi.org/10.1038/ijo.2009.289.

van den Hove, D. L. A., Van den Hove, D., Jakob, S. B., Schraut, K.-G., Kenis, G., Schmitt, A. G., et al. (2011). Differential effects of prenatal stress in 5-Htt deficient mice: towards molecular mechanisms of gene $\times$ environment interactions. PLoS One 6, e22715. doi:10.1371/journal.pone.0022715.

van der Doelen, R. H. A., Deschamps, W., D'Annibale, C., Peeters, D., Wevers, R. A., Zelena, D., et al. (2014). Early life adversity and serotonin transporter gene variation interact at the level of the adrenal gland to affect the adult hypothalamo-pituitary-adrenal axis. Transl. Psychiatry 4, e409. doi:10.1038/tp.2014.57.

van Dyck, C. H., Malison, R. T., Jacobsen, L. K., Seibyl, J. P., Staley, J. K., Laruelle, M., et al. (2005). Increased dopamine transporter availability associated with the 9-repeat allele of the SLC6A3 gene. J. Nucl. Med. 46, 745-51. Available at: http://www.ncbi.nlm.nih.gov/pubmed/15872345.

Veniaminova, E., Cespuglio, R., Cheung, C. W., Umriukhin, A., Markova, N., Shevtsova, E., et al. (2017). Autism-Like Behaviours and Memory Deficits Result from a Western Diet in Mice. Neural Plast. 2017, 1-14. doi:10.1155/2017/9498247.

Veniaminova, E., Cespuglio, R., Markova, N., Mortimer, N., Wai Cheung, C., Steinbusch, H. W., et al. (2016). Behavioral features of mice fed with a cholesterol-enriched diet:Deficient novelty exploration and unaltered aggressive behavior. Transl. Neurosci. Clin. 2, 87. doi:10.18679/CN116030/R.2016.014.

Veniaminova, E., Oplatchikova, M., Bettendorff, L., Kotenkova, E., Lysko, A., Vasilevskaya, E., et al. (2020). Prefrontal cortex inflammation and liver pathologies accompany cognitive and motor deficits following Western diet consumption in non-obese female mice. Life Sci. 241, 117163. doi:10.1016/j.lfs.2019.117163

Versteeg, R. I., Koopman, K. E., Booij, J., Ackermans, M. T., Unmehopa, U. A., Fliers, E., et al. (2017). Serotonin Transporter Binding in the Diencephalon Is Reduced in Insulin-Resistant Obese Humans. Neuroendocrinology 105, 141-149. doi:10.1159/000450549.

Wang, C.-C., Lin, H.-C., Chan, Y.-H., Gean, P.-W., Yang, Y. K., and Chen, P. S. (2013). 5-HT1A-receptor agonist modified amygdala activity and amygdala-associated social behavior in a valproate-induced rat autism model. Int. J. Neuropsychopharmacol. 16, 2027-2039. 
doi:10.1017/S1461145713000473.

Wellen, K. E., and Hotamisligil, G. S. (2003). Obesity-induced inflammatory changes in adipose tissue. J. Clin. Invest. 112, 1785-1788. doi:10.1172/JCI20514.

Wellman, C. L., Izquierdo, A., Garrett, J. E., Martin, K. P., Carroll, J., Millstein, R., et al. (2007). Impaired Stress-Coping and Fear Extinction and Abnormal Corticolimbic Morphology in Serotonin Transporter Knock-Out Mice. J. Neurosci. 27, 684-691. doi:10.1523/JNEUROSCI.4595-06.2007.

Wesołowska, A. (2010). Potential role of the 5-HT6 receptor in depression and anxiety: an overview of preclinical data. Pharmacol. Rep. 62, 564-77. Available at: http://www.ncbi.nlm.nih.gov/pubmed/20884998.

Yamamoto, M., Suhara, T., Okubo, Y., Ichimiya, T., Sudo, Y., Inoue, M., et al. (2002). Age-related decline of serotonin transporters in living human brain of healthy males. Life Sci. 71, 751-757. doi:10.1016/S00243205(02)01745-9.

Zha, W., Ho, H. T. B., Hu, T., Hebert, M. F., and Wang, J. (2017). Serotonin transporter deficiency drives estrogen-dependent obesity and glucose intolerance. Sci. Rep. 7, 1137. doi:10.1038/s41598-017-01291-5.

Zhao, Y., Li, G., Li, Y., Wang, Y., and Liu, Z. (2017). Knockdown of Tlr4 in the Arcuate Nucleus Improves Obesity Related Metabolic Disorders. Sci. Rep. 7, 7441. doi:10.1038/s41598-017-07858-6.

Zhou, L., Sutton, G. M., Rochford, J. J., Semple, R. K., Lam, D. D., Oksanen, L. J., et al. (2007). Serotonin 2C receptor agonists improve type 2 diabetes via melanocortin-4 receptor signaling pathways. Cell Metab. 6, 398405. doi:10.1016/j.cmet.2007.10.008. 


\section{Supplementary Material}

\section{Supplementary tables}

Supplementary table S1. Composition of diets used in the study.

\begin{tabular}{|c|c|c|c|c|}
\hline Diet & Weste & & Control & \\
\hline$\%$ & gm & kcal & $\mathrm{gm}$ & kcal \\
\hline Protein & 16.8 & 15 & 14.3 & 15 \\
\hline Carbohydrate & 50.5 & 43 & 72.0 & 75 \\
\hline Fat & 21.3 & 42 & 4.3 & 10 \\
\hline Total & & 100 & & 100 \\
\hline $\mathrm{kcal} / \mathrm{gm}$ & 4.61 & & 3.84 & \\
\hline Ingredient & gm & kcal & gm & kcal \\
\hline Casein, 30 Mesh & 106 & 424 & 106 & 424 \\
\hline L-Cystine & 1.6 & 6.4 & 1.6 & 6.4 \\
\hline Sucrose & 150 & 600 & 150 & 600 \\
\hline Maltodextrin 10 & 100 & 400 & 150 & 600 \\
\hline Corn Starch & 216 & 864 & 481 & 1924 \\
\hline Cellulose, BW200 & 50 & 0 & 50 & 0 \\
\hline Soybean Oil & 0 & 0 & 25 & 225 \\
\hline Palm Oil & 185 & 1665 & 20 & 180 \\
\hline Mineral Mix S10026 & 10 & 0 & 10 & 0 \\
\hline $\begin{array}{l}\text { DiCalcium } \\
\text { Phosphate }\end{array}$ & 13 & 0 & 13 & 0 \\
\hline Calcium Carbonate & 5.5 & 0 & 5.5 & 0 \\
\hline $\begin{array}{l}\text { Potassium Citrate, } \\
1 \mathrm{H} 2 \mathrm{O}\end{array}$ & 16.5 & 0 & 16.5 & 0 \\
\hline $\begin{array}{ll}\text { Vitamin } & \text { Mix } \\
\text { V10001 } & \end{array}$ & 10 & 40 & 10 & 40 \\
\hline Choline Bitartrate & 2 & 0 & 2 & 0 \\
\hline Cholesterol, NF & 1.8 & 0 & 0 & 0 \\
\hline Total & 867.45 & 3999 & 1040.65 & 3999 \\
\hline
\end{tabular}

Compared to control diet, Western diet is characterized by increased content of fat, cholesterol and sucrose and high energy density. 
Supplementary Table S2. Sequences for primers used in qPCR.

\begin{tabular}{|c|c|c|c|}
\hline Gene & $\begin{array}{l}\text { Gene } \\
\text { ID }\end{array}$ & Forward primer & Reverse primer \\
\hline Gapdh & 14433 & TGCACCACCAACTGCTTAG & GGATGCAGGGATGATGTTC \\
\hline$A c t b$ & 11461 & GGCTGTATTCCCCTCCATCG & CCAGTTGGTAACAATGCCATGT \\
\hline$B 2 m$ & 12010 & \multicolumn{2}{|c|}{ QuantiTect Primer Assays Cat. No. QT01149547 (Qiagen, Netherlands) } \\
\hline Htr1a & 15550 & AACCAGTTTTGTGTCCTCTCA & $\begin{array}{l}\text { AGCACCTAAATAATTTTCTTCT } \\
\text { C }\end{array}$ \\
\hline Htr1b & 15551 & CGCCGACGGCTACATTTAC & TAGCTTCCGGGTCCGATACA \\
\hline Htr2a & 15558 & CAGGCAAGTCACAGGATAGC & $\begin{array}{l}\text { TTAAGCAGAAAGAAAATCCCA } \\
\text { CA }\end{array}$ \\
\hline Htr2c & 15560 & $\begin{array}{l}\text { CTAATTGGCCTATTGGTTTG } \\
\text { GCA }\end{array}$ & CGGGAATTGAAACAAGCGTCC \\
\hline Htr6 & 15565 & GCATAGCTCAGGCCGTATGT & CACCACTGTGAGAGGTCCAC \\
\hline Tlr4 & 21898 & $\begin{array}{l}\text { CTGGCTAGGACTCTGATCAT } \\
\text { G }\end{array}$ & $\begin{array}{l}\text { GCATTGGTAGGTAATATTAGG } \\
\text { AACTA }\end{array}$ \\
\hline Ppargc1a & 19017 & CTCCAGTTCCGGCTCCTC & CCCTGTGCTCTCACGTCTG \\
\hline Ppargc1b & 170826 & CTCCAGTTCCGGCTCCTC & CCCTGTGCTCTCACGTCTG \\
\hline
\end{tabular}

Specific primer pairs were used in $\mathrm{qPCR}$ for gene expression measurement. 


\section{Supplementary Figures}



Supplementary Fig. S1. Daily diet intake measured in grams of diet per gram of body weight. During the $1^{\text {st }}$ week of the experiment, housing on WD led to increased daily diet intake in WT, HET and KO mice compared to mice fed with $\mathrm{CD}(\mathrm{p}<0.001, \mathrm{p}=0.044$, and $\mathrm{p}=0.003$, respectively $)$. During the $2^{\text {nd }}$ week, diet intake was decreased in KO mice fed with CD compared to WT and HET fed with $\mathrm{CD}(\mathrm{p}=0.027$ and $\mathrm{p}=0.030$, respectively) and in HET WD compared to HET CD $\left(\mathrm{p}=0.038\right.$, Tukey's). During the $3^{\text {rd }}$ week, calorie intake was decreased in KO-CD mice compared to HET-CD ( $\mathrm{p}=0.023$, Tukey's test). ${ }^{*} \mathrm{p}<0.05$, two-way ANOVA and Tukey's test, 6-7 animals per group were used. Data are shown as mean \pm SEM.
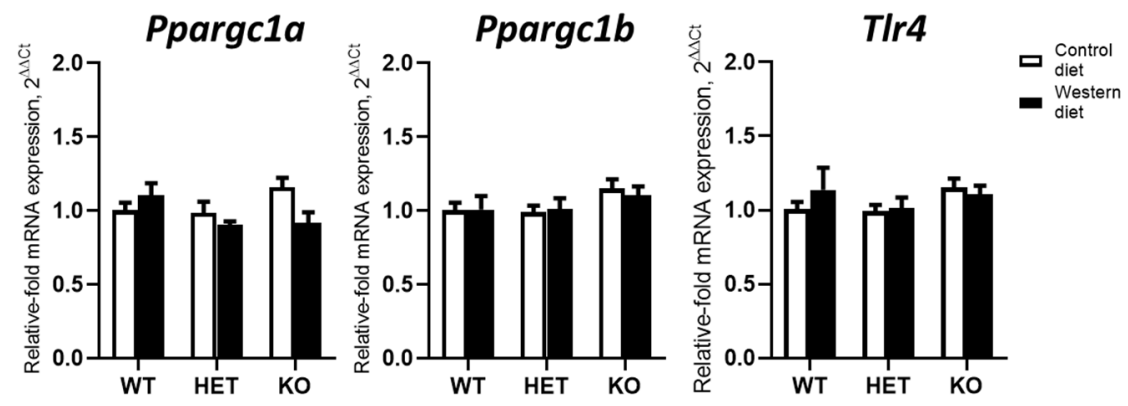

Supplementary Fig. S2. Ppargc1a, Ppargc1b and Tlr4 expression in the hippocampus. No differences in gene expression of Ppargc1a, Ppargc1b and Tlr 4 in the hippocampus were found between the groups. $\mathrm{p}>0.05$, two-way ANOVA and Tukey's test, 6-7 animals per group were used. Data are shown as mean \pm SEM. 


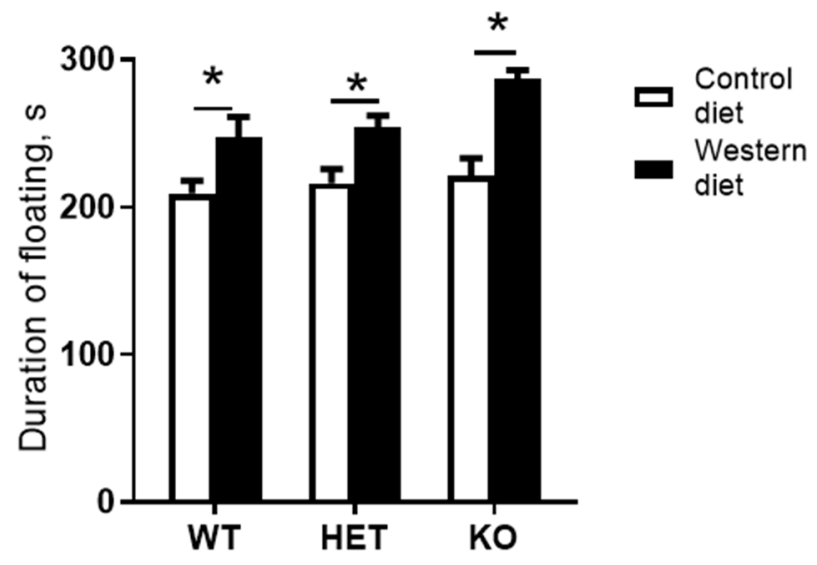

Supplementary Fig. S3. Duration of floating in forced swim test. Groups fed with WD demonstrated increased duration of floating compared to controls $\left(\mathrm{p}=0.049, \mathrm{p}=0.030, \mathrm{p}<0.001\right.$, respectively). ${ }^{*} \mathrm{p}<0.05$, two-way ANOVA and Tukey's test, 6-7 animals per group were used. Data are shown as mean \pm SEM. 
- 226 - 


\section{Chapter 5}

\section{General discussion}




\section{Chapter 5. General discussion}

5.1 Environmental factors aggravating autism spectrum disorder (ASD) and attention-deficit/hyperactivity disorder (ADHD) symptoms: focus on diet

Autism spectrum disorder (ASD) refers to a broad spectrum of conditions characterized by impaired social behaviour, communication and language, and a narrow range of interests and activities (WHO). Attention-deficit/hyperactivity disorder (ADHD) is a disorder defined by a persistent pattern of inattention and/or hyperactivity-impulsivity that interferes with functioning or development (National Institutes of Health). Both ASD and ADHD are considered to be highly heritable, however, exposure to specific environmental factors might be necessary to trigger development of the disorder (Rommelse et al., 2010). Studies have shown that there is an increased risk of ASD and ADHD in offspring due to particular prenatal and perinatal environmental exposures, such as stress, medications, chemical exposure, alcohol and tobacco exposure, maternal metabolic status, and diet (Froehlich et al., 2011; Peretti et al., 2018). The influence of maternal diet during the prenatal period on the neurodevelopment of the offspring was demonstrated both in humans and animal models (Lyall et al., 2014; Sullivan et al., 2014). ASD and ADHD demonstrate significant diagnostic overlap and shared characteristics (Taurines et al., 2012; Visser et al., 2016). These disorders co-occur with a high frequency: in 
20-50\% of children with ADHD meeting criteria for ASD and in $30-80 \%$ of ASD children meeting criteria for ADHD (Rommelse et al., 2010), with the co-occurrence found to increase with age (Rommelse et al., 2011).

While social dysfunction is a core symptom in ASD (Leitner, 2014), social difficulties are also a common feature of ADHD (Carpenter Rich et al., 2009). We found that adult female mice fed with Western diet spent less time in social contact both in a home cage and when encountered with an unfamiliar mouse (Veniaminova et al., 2017) (Chapter 2). Previously, maternal high-fat diet was shown to negatively impact offspring social behaviour in mice (Buffington et al., 2016a). Later, our findings of altered diet-induced social behaviour in the postnatal period were supported by other studies. It was reported that high-fat diet impairs social memory in adolescent rats (Reichelt et al., 2019) and reduces sociability in adult male mice (Hassan et al., 2019). Finally, Western diet, when fed from weaning to adulthood, was shown to induce increased cognitive rigidity and diminished preference for social novelty in a BTBR $\mathrm{T}+\mathrm{tf} / \mathrm{J}$ mice, which present a common model for autism (Zilkha et al., 2017).

In our model, mice fed with the Western diet were found to display behavioural abnormalities reminiscent of certain key ADHD-like symptoms: locomotor hyperactivity (Veniaminova et al., 2017) (Chapter 2), increased impulsivity (Strekalova et al., 2015), 
and emotional dysregulation in classic tests for anxiety and behavioural despair (Strekalova et al., 2016; Veniaminova et al., 2020, under revision) (Chapters 3 and 4 ). In addition, Western diet feeding led to impaired cognitive functions and motor coordination (Veniaminova et al., 2017, 2020) (Chapters 2 and 3). Motor coordination deficits were independent of changes in muscle weight, strength, or body weight. Problems with motor coordination and cognitive impairment, including difficulties with learning, have also been reported for ASD (Fournier et al., 2010; O'Brien and Pearson, 2004) and ADHD patients (Fliers et al., 2008; Mayes et al., 2000).

Thus, in our experiments, we found phenomenological similarity between the behaviours observed in mice fed with the Western diet and behaviours associated with human ASD and ADHD. Up to date, the impact of diet on ASD and ADHD in adulthood has inadequately been investigated. Several studies have reported beneficial effects of dietary interventions in children with ASD or ADHD (Heilskov Rytter et al., 2015; Pelsser et al., 2017; Sanctuary et al., 2018). Abnormally high intestinal permeability was shown in nearly $40 \%$ of autistic patients whereas this excessive permeability was present in less than $5 \%$ of normal control subjects (D'Eufemia et al., 1996). An altered gut microbiome, which can increase gut permeability, was demonstrated in ADHD (Ly et al., 2017). High intestinal permeability, which is associated with proinflammatory processes (Ding et al., 2010; Yiu et 
al., 2017), can contribute to exacerbated reactions to food components in the intestinal tract of people with ASD and ADHD. Thus, it may be hypothesized that adherence to Western dietary pattern might aggravate ADHD and ASD symptoms in adulthood, however, clinical studies are needed to investigate this hypothesis.

\subsection{Toll-like receptor 4 (Tlr4) and microglia activation in} ASD, ADHD and depression

In our model, Western diet induced ASD/ADHD-like behaviours and altered emotionality in mice, and this was accompanied by signs of neuroinflammation such as increased microglial density in prefrontal cortex (Veniaminova et al., 2020) (Chapter 3) and elevated brain expression of Tlr4 (Strekalova et al., 2015, 2016; Veniaminova et al., under revision) (Chapter 4). A growing body of evidence suggests that neuroimmune responses play an important role in the pathophysiology of mental disorders including ASD, ADHD and depression (Bennett and Molofsky, 2019).

The only way to confirm the involvement of microglia in the above-mentioned disorders in humans is to perform postmortem tissue examination or imaging studies. Assessment of microglia in postmortem brains of autism cases demonstrated cortical and cerebellum microglial activation (Morgan et al., 2010; Vargas et al., 2005). Strong activation of microglia specifically in the dorsolateral prefrontal cortex was evident, however neither microglial soma volume 
nor density showed significant correlation with ASD patient age. This suggests that microglial activation may be a long-running alteration present in developing and established ASD (Morgan et al., 2010). The early presence of microglial activation during the period of brain development indicated that it may play a central role in the pathogenesis of autism. In addition, positron emission tomography (PET) imaging, which is regarded to be the only valid method to evaluate microglial activation in the brain of living subjects, indicated excessive microglial activation in multiple brain regions in young adult patients with ASD (Suzuki et al., 2013).

When activated, microglial cells release proinflammatory cytokines and other factors contributing to neuroinflammation. Cytokine profiling indicated that MCP-1 and tumor growth factor- $\beta 1$, derived from neuroglia, were the most prevalent cytokines in ASD brain tissues (Vargas et al., 2005). CSF showed a unique proinflammatory profile of cytokines in ASD, including a marked increase in MCP-1 (Vargas et al., 2005). Also, a recent meta-analysis identified higher concentrations of IL-1 $\beta$, IL-6, IL-8, interferon-gamma, eotaxin and MCP-1 in the blood of participants with ASD compared to healthy controls (Masi et al., 2015).

So far, no direct study in postmortem brain or in living humans has investigated microglial activation associated with ADHD (Anand et al., 2017). However, there is indirect evidence that activation of 
microglia might play a role in ADHD development. ADHD symptoms were associated with increase in serum IL-16, which is found to promote recruitment of microglial cells to injured axons, and higher levels of IFN $\gamma$, which has been shown to change the protective response of microglia and macrophages into a proinflammatory response (Oades et al., 2010). The results of other peripheral cytokines levels were complex and inconsistent, but overall, they are suggestive of a low-grade inflammatory profile in patients with ADHD (Anand et al., 2017).

PET imaging revealed increased measure of microglial activation in the prefrontal cortex, anterior cingulate cortex and insula of patients with major depressive disorder (MDD) during a major depressive episode compared to healthy controls (Setiawan et al., 2015). Microglial activation measured by PET was greater in patients with chronologically advanced MDD with long periods of no antidepressant treatment than in patients with MDD with short periods of no antidepressant treatment, which is strongly suggestive of a different illness phase. Consistent with this, the yearly increase in microglial activation was no longer evident when antidepressant treatment was given (Setiawan et al., 2018). Another PET imaging study confirmed the evidence for increased microglial activation during major depressive episode, especially in patients with suicidal thoughts (Holmes et al., 2018). Postmortem studies were mainly focused on suicide victims with 
depression, demonstrating increased microglia activation in multiple brain areas (Schnieder et al., 2014; Steiner et al., 2008; Torres-Platas et al., 2014). Numerous studies have reported increases in circulating proinflammatory cytokines, IL-1, IL-6, TNF, CRP and MCP-1, in patients with major depression (Felger and Lotrich, 2013; Young et al., 2014). Some studies also observed increased proinflammatory cytokine concentrations in the CSF of depressed patients compared to controls or correlations between CSF cytokines and depression severity (Levine et al., 1999; Lindqvist et al., 2009; Martinez et al., 2012).

Microglia cells express numerous members of the TLR family, including TLR4 (Kielian, 2006). TLR4 has been reported to induce microglial activation and cytokine production, promoting inflammation, which in turn may positively regulate increased expression of TLR4 (Kielian, 2006). Mice fed with the Western diet demonstrated increased TLR4 expression together with impairment in social, cognitive, motor, and emotionality-related behaviours (Strekalova et al., 2016; (Veniaminova et al., 2017, 2020, under revision) (Chapters 2, 3 and 4). In agreement with our results, other animal studies suggested involvement of TLR4-dependent signaling in the development of ASD/ADHD-like behaviours. Embryos and newborns of BTBR $\mathrm{T}+\mathrm{tf} / \mathrm{J}$ mice demonstrated increased Tlr4 and proinflammatory cytokine expression both in blood and in the brains compared to wild type animals (Cipriani et al., 2018). Improvement of 
ASD-like behaviour in adult BTBR $\mathrm{T}+\mathrm{tf} / \mathrm{J}$ mice after treatment with natural phenol resveratrol was accompanied by decreased Tlrs, including Tlr4, and NF-xß expression (Ahmad et al., 2018). Tlr4 knockout mice displayed altered emotional and social behaviour, further supporting that TLR4 signaling is involved in emotional regulation (Femenia et al., 2018)

Moreover, ASD individuals demonstrated increased TLR4 expression on T cells (Nadeem et al., 2017), and higher impairment in social behaviours as well as non-verbal communication were associated with increased production of IL-1 $\beta$ and IL-6 after TLR4 stimulation (Enstrom et al., 2010). Altered TLR responses, including increased production of proinflammatory cytokine IL-23 in blood mononuclear cells in response to TLR4 agonist LPS, were found in a subset of ASD children characterized by frequent infections and by worsening behavioural symptoms following infection (Jyonouchi et al., 2008). NF- $x \mathrm{~B}$ upregulation had been found in the postmortem prefrontal cortex in ASD and depression patients (Malki et al., 2015; Young et al., 2011). Upregulated TLR4 expression was demonstrated in peripheral blood mononuclear cells in newly diagnosed patients with MDD (Kéri et al., 2014) and in brain tissue of depressed subjects, compared with controls (Pandey et al., 2014). While the involvement of TLR4-dependent signaling was not directly investigated in ADHD patients, a genome-wide association study found an increased burden 
of genetic variation in the genes involved in TLR signaling to be associated with ADHD (O'Dushlaine et al., 2015). Exploration of the possible role of TLR4 in pathogenesis of ADHD requires further clinical studies.

In summary, based on clinical studies and postmortem patient examination, it can be suggested that microglial activation and TLR4 signaling play a significant role in the mechanisms of ASD, depression and, likely, ADHD (Table. 5.1). Our results in an animal model of the Western diet feeding are in line with these data, further supporting the hypothesis of a poor diet as a key environmental factor contributing to the development of mental disorders.

Table 5.1. Clinical data supporting the involvement of TLR and microglia activation in mechanisms of ASD, ADHD and depression.

\begin{tabular}{|l|l|l|}
\hline Disorder & Microglial activation & TLR activation \\
\hline ASD & $\begin{array}{l}\text { Postmortem examination } \\
\text { PET imaging }\end{array}$ & $\begin{array}{l}\text { Altered TLR responses } \\
\uparrow \text { TLR4 on T cells, } \uparrow \text { NF- } \varkappa \mathrm{B}\end{array}$ \\
\hline ADHD & $\begin{array}{l}\text { No direct examination } \\
\uparrow \text { serum IL-16 and INF } \gamma\end{array}$ & $\begin{array}{l}\text { No direct examination } \\
\text { Polymorphisms in genes } \\
\text { involved in TLR signaling }\end{array}$ \\
\hline Depression & $\begin{array}{l}\text { Postmortem examination } \\
\text { PET imaging }\end{array}$ & $\begin{array}{l}\uparrow \text { TLR4 in brain and blood } \\
\uparrow \text { NF- } \varkappa \mathrm{B}\end{array}$ \\
\hline
\end{tabular}

In patients with ASD and depression, postmortem brain examination and PET imaging revealed marked microglia activation. To date, there is only indirect evidence that activation of microglia might play a role in ADHD development, such as increased levels of IL-16 and IFN $\gamma$, as no direct study has investigated microglial activation in ADHD patients. Patients with ASD demonstrate altered TLR responses and both in ASD and depression TLR and NF- $x \mathrm{~B}$ are upregulated. Polymorphisms in genes related to TLR signaling were detected in ADHD subjects. 


\subsection{Oxidative stress and mitochondrial dysfunction in ASD, ADHD and depression}

An increase in oxidative stress and a decrease in the antioxidant capacity of the brain are suggested as key factors involved in the mechanisms of neuropsychiatric diseases (Popa-Wagner et al., 2013). ROS can produce membrane damage, affect the structure and function in the inner proteins, cause denaturation of lipids and structural damage to DNA. Mitochondria is the most important source of ROS. The brain, being the major metabolizer of oxygen and containing a large amount of polyunsaturated peroxidizable fatty acids, is especially vulnerable to oxidative stress (Popa-Wagner et al., 2013). Oxidative stress can impair neuronal proliferation and mediate apoptosis, and therefore lead to progressive neuronal damage and disturbance of normal brain functions (Kannan and Jain, 2000; Klein and Ackerman, 2003). The levels of several metabolites and activity of enzymes altered in brain tissue and serum can be used as potential biomarkers of central and peripheral oxidative stress.

The Western diet in our model induced an increase in the level of marker for lipid peroxidation malondialdehyde (MDA) (Veniaminova et al., 2020) (Chapter 3) and decrease in the expression of mitochondrial biogenesis regulators peroxisome proliferatoractivated receptor gamma coactivator (Ppargc) 1a and b in mouse brain (Strekalova et al., 2016; Veniaminova et al., 2017, under revision) 
(Chapters 2 and 4). These changes are the signs of oxidative stress and impaired mitochondrial functioning in brain. Western diet feeding in mice also induced behaviours reminiscent of several symptoms of ASD, ADHD and depression. Taken together, the data on the involvement of mitochondrial dysfunction and oxidative stress in ASD, ADHD and depression would strengthen our hypothesis on the role of a dietary factor in the mechanisms of these disorders.

Reports indicate increased levels of lipid peroxidation markers in ASD patients, thus confirming an increased level of oxidative stress in autism. Compared to healthy controls, autistic subjects demonstrated elevated MDA in plasma (Chauhan et al., 2004) and erythrocytes (Zoroglu et al., 2004). Increased levels of NO, a toxic free radical that reacts with the superoxide anion and generates cytotoxic peroxynitrate anions (ONOO-), have been reported in red blood cells of patients with autism and have suggested that nitric oxide synthase (NOS) may be activated in autism (Söğüt et al., 2003). Elevated plasma levels of nitrite and nitrate were also shown in ASD patients (Sweeten et al., 2004; Zoroğlu et al., 2003). Increased activity of xanthine oxidase (XO), which is an endogenous prooxidant, has been reported in the erythrocytes of patients with ASD (Zoroglu et al., 2004).

Several studies have suggested alterations in the enzymes that play an important role in the defense mechanism against damage by ROS in autism. Compared to healthy control, ASD patients exhibited 
significantly lower total antioxidant status (TAS), which represents the cumulative effect of all antioxidants, in urine (Yui et al., 2017) and serum (Ozturk et al., 2016). Patients with autism showed decreased activity of glutathione peroxidase in plasma and erythrocytes (Paşca et al., 2006; Yorbik et al., 2002), as well as reduced levels of total glutathione and lower redox ratio of reduced glutathione to oxidized glutathione (GSH/GSSG) in plasma (James et al., 2004). Decreased catalase and superoxide dismutase (SOD) activity in erythrocytes has also been indicated in individuals with autism (Yorbik et al., 2002). Consistent with studies on plasma and immune cells, GSSG and GSH/GSSG were significantly decreased in both cerebellum and temporal cortex of ASD patients (Rose et al., 2012). There was also a significant increase in biomarkers of oxidative protein damage, 3-nitrotyrosine (3-NT), and oxidative DNA damage, 8-oxo-deoxyguanosine (8-oxo-dG), in the ASD brain (Rose et al., 2012).

Mitochondrial DNA (mtDNA) copy number is a strong biomarker for mitochondrial dysfunction, since it may be increased with mtDNA damage or mitochondrial dysregulation to compensate for impaired mitochondrial energy metabolism. In several reports, the mtDNA copy number was increased in the peripheral blood and prefrontal cortex of ASD patients (Gu et al., 2013; Yoo et al., 2017). Likewise, activities of electron transport chain complexes and pyruvate dehydrogenase $(\mathrm{PDH})$ were significantly reduced in muscle tissue, blood and brain, 
including cerebellum and prefrontal cortex (Chauhan et al., 2011; Gu et al., 2013; Guevara-Campos et al., 2010; Oliveira et al., 2005).

PPARGC1a and b are important regulators of mitochondrial fatty acid oxidation, which is impaired in ASD. These co-activators bind and activate a number of transcription factors, including the nuclear peroxisome proliferator-activated receptors (PPARs), and, accordingly, mediate a variety of stimulatory effects on mitochondrial bioenergetics across multiple organs, including the brain (Lin et al., 2005; Nierenberg et al., 2018). Various studies document the presence of PPARs in neurons and glial brain cells and support the role of these nuclear receptors in neuroprotection (Agarwal et al., 2017; Tontonoz and Spiegelman, 2008). Evidence suggests the effectiveness of PPAR $\alpha$ activation in amelioration of ASD-like symptoms in BTBR $\mathrm{T}+\mathrm{tf} / \mathrm{J}$ mice (D'Agostino et al., 2015). In addition, PPAR $\alpha$-deficient mice have a distinct cognitive and behavioural phenotype characterized by reduced spatial information processing and cognitive flexibility, along with repetitive behaviour reminiscent of that seen in preclinical models of ASD (D'Agostino et al., 2015). Finally, the use of PPAR $\gamma$ activator pioglitazone was effective in the amelioration of behavioural symptoms of ASD in clinical trials (Ghaleiha et al., 2015).

Various studies demonstrated elevated levels of peripheral oxidative damage markers in ADHD. For example, increased lipid peroxidation was shown in ADHD reflected by raised urinary acrolein- 
lysine levels and by breath ethane levels (Kawatani et al., 2011; Ross et al., 2003). Results on MDA are inconsistent, with higher (Bulut et al., 2007; Ceylan et al., 2010) as well as lower plasma levels found in ADHD patients compared to controls (Oztop et al., 2012). Higher serum xanthine oxidase $(\mathrm{XO})$ activity was observed in paediatric ADHD patients (Ceylan et al., 2012). In addition, NO levels and NOS activity were higher in ADHD patients than in controls, but while NOS activity was positively correlated with teacher hyperactivity ratings, ADHD subtype did not correlate with NO level or NOS activity (Ceylan et al., 2010, 2012). Total DNA damage in ADHD as compared to controls was increased as determined by the concentration of 8-oxo7,8-dihydroguanine (8-oxoG), the oxidized product of a free guanine base, in lymphocytes (Chovanová et al., 2006). Finally, higher total oxidant status (TOS) representing the cumulative effect of all oxidants present in the investigated sample and oxidative stress index (OSI) were found in ADHD (Guney et al., 2015; Kul et al., 2015; Sezen et al., 2016).

Plasma and saliva activity levels of the antioxidant enzymes glutathione-S-transferase (GST), SOD and catalase (CAT) were decreased in children and adolescents with ADHD as compared to controls, indicating lower antioxidant activity (Ceylan et al., 2010, 2012; Ruchi et al., 2011; Russo, 2010). Evidence concerning thiol levels is conflicting, as lower and higher levels were reported in plasma and 
serum of ADHD patients compared to controls (Alpak et al., 2014; Avcil et al., 2017; Guney et al., 2015). Finally, many studies have reported lower TAS in ADHD as compared to healthy participants (Kul et al., 2015; Ruchi et al., 2011; Sezen et al., 2016). Higher TAS and no difference have also been reported (Guney et al., 2015). It should be mentioned that higher antioxidant levels in some cases might be attributed to a reactive, compensatory increase due to protection against increased oxidative stress (Guney et al., 2015; Oztop et al., 2012).

Several recent studies showed mitochondrial dysfunction in ADHD pathogenesis. Lower mitochondrial respiration, lower ATPase 6/8 transcripts levels, reduced mitochondrial complex $\mathrm{V}$ activity and loss of mitochondrial membrane potential were reported in cybrids from ADHD patients compared to healthy controls (Verma et al., 2016). Another study showed a significant association of mtDNA 10398 A/G polymorphism with ADHD in children (Hwang et al., 2017). Relative mtDNA copy number was significantly higher and mtDNA methylation ratio of PPARGC1A was decreased in ADHD patients compared to healthy volunteers (Kim et al., 2019).

Oxidative stress-related damage has been described extensively in depression and, unlike the data on ASD and ADHD obtained mostly from blood or peripheral tissue, many studies were done using brain tissue. Membrane damage was shown in the blood of patients with 
depression by elevated lipid peroxidation products (Peet et al., 1998; Sarandol et al., 2007). In patients with depression, elevated levels of MDA were found to adversely affect cognitive function and were associated with increased severity of depressive symptoms (Talarowska et al., 2012). Antidepressant administration decreased MDA levels (Gałecki et al., 2009; Khanzode et al., 2003). In addition, serum levels of a marker of lipid peroxidation 8-isoprostane-F2alpha were shown to be higher in patients with MDD compared to healthy controls (Yager et al., 2010). Cerebral membrane abnormalities and altered membrane phospholipids have been suggested by an increased choline-containing compound seen in the putamen of patients with depression which has been interpreted as a result of increased oxidative stress (Ende et al., 2007).

One report of unmedicated patients with depression found decreased TAS in plasma as well as increased total plasma peroxide and OSI compared to healthy control subjects (Yanik et al., 2004). The activity of antioxidant enzymes in patients with depression is controversial. Both increased and decreased activity of SOD has been described (Kodydková et al., 2009; Lukic et al., 2014). In the frontal cortex, $\mathrm{Cu} / \mathrm{Zn}-\mathrm{SOD}$ concentration was increased in patients with depression compared to matched controls (Michel et al., 2007). $\mathrm{Cu} / \mathrm{Zn}-\mathrm{SOD}$ is mainly located in glial cells, which are more vulnerable to oxidative damage (Papadopoulos et al., 1997). There was also a 
significant increase in XO activity in some brain areas of patients with MDD compared to non-affected controls (Michel et al., 2010). Lowered activity of glutathione peroxidase, which causes accumulation of ROS, was detected in patients with depression (Maes et al., 2011). Some studies on the other hand did not find significantly different level of glutathione peroxidase in depressed patients (Gałecki et al., 2009; Lukic et al., 2014). In urine of depressed patients, elevated levels of 8-OxoG were detected compared to healthy controls (Maes et al., 2009), suggestive of oxidative DNA damage.

Depression is accompanied by mitochondrial disturbances, such as lower activities of respiratory chain enzymes and ATP production (Gardner, 2003). Findings in brains of depressed patients demonstrated altered expression of mitochondrial-located proteins in the anterior cingulate cortex (Beasley et al., 2006) and complex I subunits in the cerebellum (Ben-Shachar and Karry, 2008). MtDNA copy number was increased in patients with depression and anxiety and the change in mtDNA copy number after treatment was associated with the treatment response (Wang et al., 2017). In addition, PPAR $\gamma$ and $\operatorname{PPAR} \alpha$ activation was demonstrated as a promising therapy in affective disorders (Colle et al., 2016; Nisbett and Pinna, 2018).

In summary, patients with depression, ASD and ADHD demonstrate similar oxidative-stress related impairments both in peripheral tissue and the brain, suggesting shared pathophysiological 
mechanism associated with mitochondrial dysfunction. Specifically, the mitochondria in individuals with these disorders may produce elevated quantities of ROS, increasing brain vulnerability to oxidative damage. When the ability of the antioxidant machinery to balance ROS production fails, oxidative stress predominates and leads to further mitochondrial (and cellular) dysfunction (Fig. 5.1). Our findings in the model of Western diet were similar to the clinically derived associations between increased lipid peroxidation, decreased mitochondrial biogenesis and ASD/ADHD-like and depressive-like behaviours. This suggests the Western diet as a potential contributor to the development of these disorders.

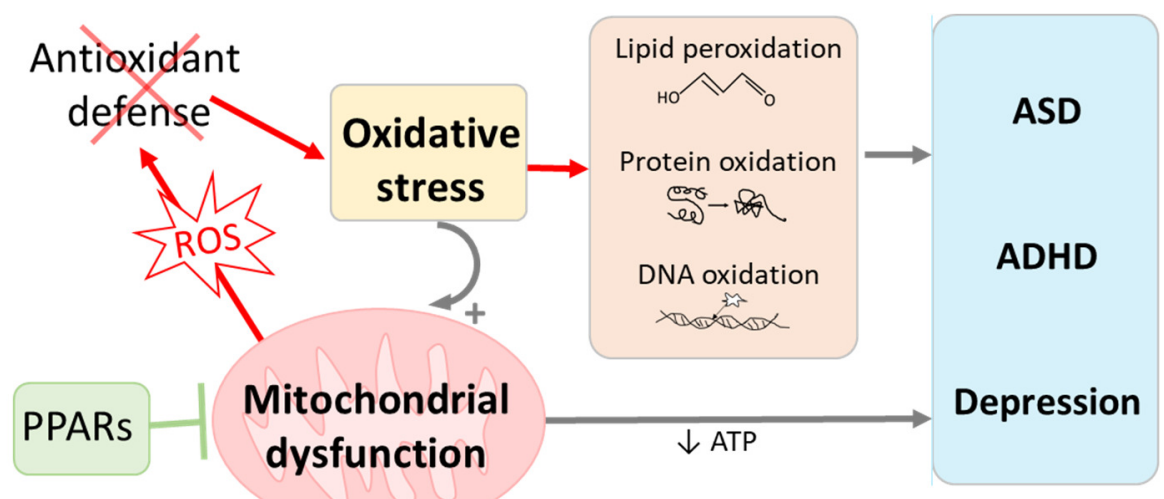

Figure 5.1. Oxidative stress and mitochondria dysfunction as factors contributing to ASD, ADHD and depression development. Mitochondria produce elevated quantities of ROS increasing brain vulnerability to oxidative damage. When the ability of the antioxidant machinery to balance ROS production fails, oxidative stress predominates and leads to further mitochondrial dysfunction. Increased lipid peroxidation, protein and DNA oxidation results in neuronal damage and disturbance of normal brain functions contributing to the development of mental disorders, including ASD, ADHD and depression. 


\subsection{Interplay between peroxisome proliferator-activated receptors (PPARs), Tlr4, serotonin transporter and insulin receptors activity}

The Western diet in our mouse model induces increases in Tlr4 expression and microglial activation, elevated MDA level (Veniaminova et al., 2020, under revision; Strekalova et al., 2015, 2016), decreased expression of Ppargc1a and Ppargc1b (Strekalova et al., 2016; Veniaminova et al., 2017) as well as a decrease in serotonin transporter expression (Sert) (Veniaminova et al., 2020) in the brain (Chapters 2-4). Importantly, increased protein level of Tlr4, microglial activation, elevated MDA content and decreased Sert mRNA level were detected in the prefrontal cortex of young mice fed with the Western diet (Veniaminova et al., 2020; Strekalova et al., 2015). These data led us to suggest that these brain changes did not happen independently but are interconnected, influencing each other and resulting in the alteration of mouse behaviour.

Both TLRs and PPARs are involved in modulating the inflammation responses effecting the same molecular pathways (Dana et al., 2019). Signaling pathways of most of the TLRs, including TLR4, operate through MyD88 and are accompanied by a TIR domaincontaining adaptor protein (TIRAP), which results in activation of transcription factors including NF- $\varkappa \mathrm{B}$ and activator protein-1 (AP-1) (Troutman et al., 2012). NF- $\varkappa \mathrm{B}$ and AP-1 pathways regulate many inflammation-related genes. PPARs can attenuate the expression of 
inflammatory response genes by inactivation of p65 complexes or through inducing $\mathrm{I} \chi \mathrm{B} \alpha$ which is the main inhibitor of $\mathrm{NF}-\varkappa \mathrm{B}$ signaling pathway. It was also shown that PPARs can regulate AP-1 activity (Delerive et al., 2001). Studies in cell culture and animal models demonstrated that there is bidirectional regulation between TLRs and PPARs (Dana et al., 2019). One study described a regulatory feedback loop in which in unstimulated macrophages, PPAR $r$ inhibited NF- $x$ B-mediated inflammatory signaling, while in LPS-stimulated macrophages, when the TLR4 was activated, the PPAR $\gamma$ expression was attenuated by NF- $\chi \mathrm{B}$ and as a result, any further potential antiinflammatory effects of PPAR $\curlyvee$ were terminated (Necela et al., 2008). Moreover, PPAR $\gamma$ agonists were shown to inhibit TLR-mediated activation of dendritic cells via the MAP kinase and NF- $x \mathrm{~B}$ pathways (Appel et al., 2005). In a murine model of sepsis, PPAR $\gamma$ activation by an agonist resulted in attenuated proinflammatory cytokine production and increased level of IL-10. These effects were associated with a decrease in STAT-1-dependent expression of MyD88. Blocking the IL-10 receptor was able to eliminate PPAR $\gamma$-mediated inhibition of MyD88 expression (Ferreira et al., 2014).

Glial PPAR $\alpha$ and $\gamma$ play an important role in inflammatory brain pathologies. PPAR $\gamma$ agonists were shown to attenuate microglia activation, TLR4 and cytokines expression (Jia et al., 2016) and TLR ligand-induced production of IL-12 p40 (Gurley et al., 2008). Also, 
agonists of TLR4, TLR1/2 and TLR5 in astrocytes demonstrated $\mathrm{NF}-x \mathrm{~B}$-dependent inhibitory activity against gene expression, protein translation and the activity of PPAR $\alpha$ and PPAR $\gamma$ (Chistyakov et al., 2015). Protective anti-inflammatory efficiency of PPAR $\beta / \delta$ agonists was shown in animal models (Aleshin and Reiser, 2013). However, the fact that PPAR $\beta / \delta$ expression levels were found to be upregulated after TLR4 stimulation through NF- $x \mathrm{~B}$ and MAPK-dependent pathways, raised a question of whether $\operatorname{PPAR} \beta / \delta$ has a dual pro- and antiinflammatory role (Chistyakov et al., 2014).

Inflammation-related regulation was demonstrated for serotonin transporter (SERT) expression. Thus, in cell culture SERT expression and activity were inhibited by activation of TLR2 via MAPK and protein kinase A (PKA) pathways (Latorre et al., 2016) and by activation of TLR4 mediated by protein kinase C (PKC) (Mendoza et al., 2009). On the other hand, individuals carrying two short (ss) versus long (ll) 5-HTTLPR alleles showed a higher ratio of proinflammatory to anti-inflammatory cytokines IL-6/IL-10 at baseline and during stress (Fredericks et al., 2010). In the rat brain, both inhibition of SERT by fluoxetine (Macgillivray et al., 2011) and genetic Sert deficiency (Macchi et al., 2013) resulted in microglia activation. However, in the other study, systemic LPS led to increased Sert function in male rats (Korte-Bouws et al., 2018). In addition, serotonin metabolites, 5methoxyindole-3-acetic acid and 5-hydroxyindoleacetic acid (5-HIAA), 
can directly activate PPAR $\gamma$ and this process was inhibited by blocking of endogenous SERT (Waku et al., 2010). Treatment with a $\operatorname{PPAR} \beta / \delta$ agonist was shown to enhance the expression of $\operatorname{PPAR} \beta / \delta$ and Sert in the hippocampus of rats with stress-induced depression (Liu et al., 2017a). Metabolic endotoxemia induced by decreased SERT in the gut might lead to inhibition of peripheral and brain IRS1/PI3K/Akt regulatory cascades which is a key mechanism in the regulation of glucose metabolism (Pomytkin et al., 2015).

Both chronic inflammation and alterations in mitochondrial biogenesis are implicated in the mechanisms of insulin resistance (Pomytkin et al., 2015; Zand et al., 2017). TLR4 is involved in insulin resistance-associated inflammation via activating proinflammatory kinases JNK, IKK, and p38 that impair insulin signal transduction directly through inhibitory phosphorylation of IRS on serine residues. (Kim and Sears, 2010; Yin et al., 2014). In addition, activation of TLR4 leads to phenotypic change of macrophage from M2 to M1. The proinflammatory phenotype of M1 macrophages further promote insulin resistance (Orr et al., 2012). Some studies found increased TLR4 mRNA expression and signaling in type 2 diabetic patients (Creely et al., 2007; Jialal et al., 2012; Lee et al., 2013). Also, increased $\mathrm{NF}-x \mathrm{~B}$ activation may lead to decreased insulin receptor-facilitated insulin uptake by the brain (Gray et al., 2017). 
PPARGC1a-responsive genes involved in oxidative phosphorylation were shown to be downregulated in human diabetes (Mootha et al., 2003). PPARs can affect insulin signaling by decreasing production of proinflammatory mediators and NF-kB transcriptional activities and upregulation of IRS proteins (McArdle et al., 2013; Wahli and Michalik, 2012). In addition, PPAR $\curlyvee$ was demonstrated to promote alteration of immune cells phenotypes to anti-inflammatory M2 macrophage polarization (Patel et al., 2013), thus antagonizing TLR4-dependent effects.

It is important to mention that previous studies conducted in our model revealed ameliorative effects of insulin receptor sensitizer dicholine succinate on Western diet-induced elevation of Tlr4 and decrease of Ppargc1b brain expression in mice (Strekalova et al., 2016). Recent unpublished data demonstrate that in Sert knockout mice, Western diet induces a decrease in the expression of both isoforms of insulin receptor, IRA and IRB, in the brain (Veniaminova et al., unpublished data; Fig. 5.2). Interestingly, in naïve Sert knockout mice, IRA and IRB expression was elevated compared to wild type and Sert heterozygous mice, possibly demonstrating adaptive changes due to the absence of Sert. 
IRA

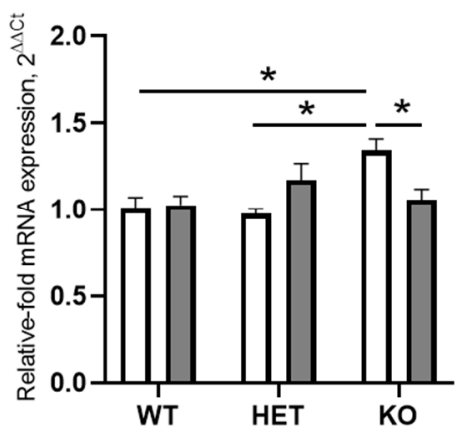

IRB



Figure 5.2. IRA and IRB expression in Sert knockout mice fed Western or control diet. Sert knockout mice fed with the Western diet compared to control diet demonstrated decreased expression of IRA and IRB in hypothalamus. Naïve Sert knockout mice compared to wild type and heterozygous showed increase in IRA and IRB expression. Similar results were obtained for dorsal raphe region, hippocampus and prefrontal cortex (not shown). WT, wild type; HET, Sert heterozygous, KO, Sert knockout mice. ${ }^{*} \mathrm{p}<0.05$, two-way ANOVA and Tukey's test, 6-7 animals per group were used. Data are shown as mean \pm SEM.

Thus, there is a complicated crosstalk involving TLRs, PPARs, SERT and insulin receptor signaling. TLR4 and PPARs have shared signaling pathways, antagonizing each other. Experimental data suggest that activation of TLR and inhibition of PPARs and insulin receptor-mediated signaling may lead to impaired functioning both in the periphery and in the brain. 


\subsection{Direct and peripheral inflammation-mediated effects of Western diet components on neuroinflammation and brain mitochondrial activity}

In our model, Western diet enriched with fats, especially saturated fats, and sugar, was shown to induce changes both in peripheral metabolism and in the brain in female mice. Among the peripheral changes, we found impaired glucose tolerance and insulin resistance, dyslipidemia, and signs of liver steatosis and inflammation in mice fed with the Western diet compared to controls. In the brain, Western diet induced signs of neuroinflammation and impaired mitochondrial activity (Veniaminova et al., 2017, 2020, under revision) (Chapters 2-4). While we did not investigate exact molecular cascades connecting Western diet consumption with brain changes, there are two pathways that possibly contribute to the diet effects on the brain.

First, components of the Western diet can induce peripheral inflammation-related metabolic states, which in turn may lead to neuroinflammation (discussed in section 1.2). In human, consumption of excess sugar or fat can cause overeating, increased body weight and fat gain (Stinson et al., 2018). Enlarged adipocytes of obese individuals are known to promote inflammation and predispose toward insulin resistance (Greenberg and Obin, 2006). Some studies have found an association between the consumption of added sugar or fat and development of insulin resistance and type 2 diabetes, independent of 
body weight gain or total energy intake (Marshall and Bessesen, 2002; Stanhope, 2016). On the contrary, others argue that much of the association between sugars and type 2 diabetes is eliminated by adjusting data for body mass index (Lean and Te Morenga, 2016; Marshall and Bessesen, 2002), which makes this topic controversial. In our study, Western diet feeding induced impairment in glucose tolerance both in the presence of weight gain in aged mice (Veniaminova et al., under revision) (Chapter 4) and in young mice without a change in body weight (Veniaminova et al., 2017, 2020) (Chapters 2 and 3).

Altered diet can lead to rapid, short-term changes in hepatic glucose metabolism. In healthy people, fructose and glucose overfeeding for 6 days increased liver fat content and reduced hepatic insulin sensitivity (Lecoultre et al., 2013). Sucrose stimulates de novo lipogenesis which leads to the alteration of the fatty acid composition in very-low-density lipoprotein (Hoekstra et al., 1993). Dietary intake of lipids, the lipolysis of adipose tissue, and de novo lipogenesis contribute to the pool of lipids stored in the liver and to the development of non-alcoholic fatty liver disease (Marchesini et al., 2016). Western diet feeding resulted in liver steatosis and inflammation, demonstrated in our study (Veniaminova et al., 2020) (Chapter 3) and has been previously shown to decrease Ppargc1b liver expression (Strekalova et al., 2016). In line with our findings, 
PPARס was shown to suppress hepatic lipogenesis and reduce the hepatic expression of proinflammatory genes (Tong et al., 2019). In addition, increased fructose or SFA levels can lead to a higher gut permeability via disruption of the tight junctions (de Velasco et al., 2018; Johnson et al., 2013). The resulting endotoxemia leads to further systemic low-grade inflammation.

Secondly, circulating fatty acids and glucose may also directly affect the brain. Two main types of transporters facilitate glucose transport across the BBB: glucose transporters (GLUTs) and sodiumdependent glucose transporters (SGLTs) (Patching, 2017). There is no consistency in reported effects of blood hyperglycemia on activity and expression of glucose transporters, as upregulation, downregulation and no changes have been shown (Patching, 2017). No major adaptational changes in the maximal transport velocity or affinity to BBB glucose transporters were found under conditions of acute hyperglycemia in normal human subjects (Hasselbalch et al., 2001). In rats, chronic hyperglycemia was shown to elevate brain extracellular fluid glucose, suggesting no protective adaptation in BBB (Jacob et al., 2002). Hence, the brain tissue may be exposed to elevated levels of glucose. In addition, glucose influx in the brain may be increased due to increased BBB permeability, induced by peripheral inflammation (Van Dyken and Lacoste, 2018). Once in the brain, glucose in extracellular fluid is rapidly taken up by astrocytes, microglia and neurons. Elevated 
glucose concentrations were shown to increase secretion of IL-6 and IL-8 in cultured human astrocytes and increased the susceptibility of human neuronal cells to injury by hydrogen peroxide (Bahniwal et al., 2017). In mouse microglial cells, a shift from normal glucose to high glucose induced oxidative and inflammatory stress, microglial activation and increased LPS-induced inflammation (Hsieh et al., 2019). Changes in glucose level activated MAPK/JNK and NF- $x \mathrm{~B}$ signaling pathways (Fig. 5.3). This way, acute glucose fluctuation altered microglial activity, inducing oxidative and proinflammatory changes (Hsieh et al., 2019). This study is in line with our findings, as consumption of the Western diet in mice, which might lead to fluctuations in glucose levels, also induced microglial activation and oxidative stress (Veniaminova et al., 2020) (Chapter 3).

Numerous studies have discussed the transport of both saturated fatty acids (SFA) and unsaturated fatty acids across the BBB. Fatty acids can enter the brain in a number of ways. They can be transported via lipoproteins or bound to albumin in esterified or non-esterified form. Some may enter via passive diffusion, through a flip-flop mechanism or through protein transporters (Chen et al., 2015; Hachem et al., 2016; Murphy, 2015; Nguyen et al., 2014; Umhau et al., 2009). Several studies have reported that SFAs, such as palmitic acid, can induce activation of TLR4 receptors and stimulate cytokine release (Caesar et al., 2015; Nguyen et al., 2007; Valdearcos et al., 2014). 


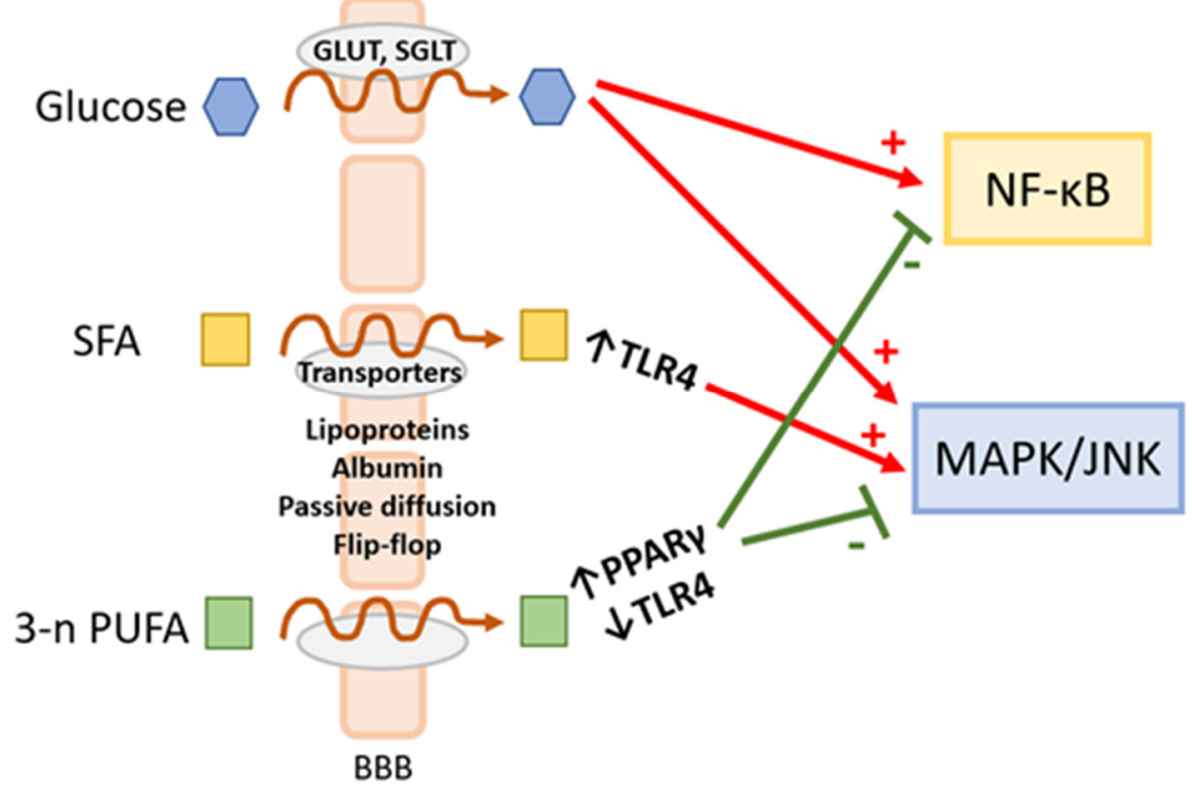

Figure 5.3. Direct effects of fatty acids and glucose on signaling in the brain. Glucose is transported across the BBB by GLUTs and SGLTs and activates MAPK/JNK and NF- $\chi \mathrm{B}$ signaling pathways. Fatty acids enter the brain via lipoproteins or bound to albumin, via passive diffusion, through a flip-flop mechanism or through protein transporters. SFAs induce JNK signaling activation and increased inflammation, which is regulated by TLR4. n-3 PUFAs can inhibit TLR4 presentation and induce PPAR $\gamma$ nuclear translocation, which prevents $\mathrm{NF}-x \mathrm{~B}$ and MAPK phosphorylation

It has been previously hypothesized that long-chain SFAs are TLR4 agonists (Osborn and Olefsky, 2012). The principal component of LPS responsible for its immunostimulatory activity is the lipid A region, which contains numerous saturated fatty acyl chains that are required for binding to and activating TLR4 (Park and Lee, 2013). However, it was demonstrated that long-chain SFAs take several hours to initiate inflammatory signaling, while LPS activates inflammatory 
signaling within minutes (Hernandez et al., 2014; Holzer et al., 2011; Nguyen et al., 2007). Recently, Lancaster et al. (2018) demonstrated that SFA palmitic acid is not a TLR4 agonist. Rather, they provided evidence that TLR4 indirectly regulates SFA-induced inflammation by altering macrophage lipid metabolism. Specifically, metabolic reprograming facilitates SFA effects on endoplasmic reticulum, which leads to JNK signaling activation and increased inflammation (Lancaster et al., 2018). Nevertheless, as SFA-induced inflammation is regulated by TLR4, it provides a link between increased brain expression of Tlr4 and high content of saturated fat in the Western diet in our study.

On the contrary, n-3 polyunsaturated fatty acids (PUFAs), such as docosahexaenoic acid (DHA) and eicosapentaenoic acid (EPA), typically low in the Western diet, were shown to decrease circulating inflammatory markers and reduce oxidative stress (Freund-Levi et al., 2006; Kiecolt-Glaser et al., 2012). When incorporated into microglial membranes, n-3 PUFAs can prevent LPS-induced NF- $x \mathrm{~B}$ activation by inhibiting TLR4 presentation and reduce NO production, inducible nitric oxide synthase expression and the overall level of oxidative stress (Antonietta Ajmone-Cat et al., 2012; Fourrier et al., 2017; Inoue et al., 2017). Moreover, DHA might induce PPAR $\curlyvee$ nuclear translocation and prevent LPS-induced MAPK phosphorylation (Antonietta AjmoneCat et al., 2012). It was proposed that DHA also attenuates the 
inflammatory response in LPS-activated microglia by being incorporated into and remodeling lipid bodies, dynamic organelles that serve many functions, including to assist in preserving cell membrane and mitochondrial integrity (Tremblay et al., 2016). EPA also was shown to inhibit NF- $\varkappa \mathrm{B}$ phosphorylation via sirtuin-1 (Inoue et al., 2017; Moon et al., 2007). In addition, DHA and EPA are able to enhance myelin or amyloid $\beta$ peptide phagocytosis that is associated with a shift in microglial polarization toward the beneficial M2 phenotype, with a concomitant decrease in proinflammatory cytokine production (Chen et al., 2015; Hjorth et al., 2013). PUFA metabolites, such as resolvins, can limit the activation of microglial cells and inhibit the TLR4/NF $x$ B pathway (Tian et al., 2015).

Based on this evidence from prior research, it can be suggested that the effects of Western diet in mice could be mediated both by peripheral inflammation and associated systemic metabolic changes, as well as the direct interaction between diet components and brain molecular pathways related to TLR4 and PPARs.

\subsection{The role of Sert deficiency in the interaction between genetic predisposition to psychiatric disorders and diet}

Gene-environment interaction plays an important role in the development of psychiatric disorders (Schmitt et al., 2014). Dietary factors, such as Western-pattern diet consumption, are proposed among those environmental factors which could interact with genetic 
susceptibility. Genetic Sert deficiency in human induced by s variant in the 5-HTTLPR of the SERT (SLC6A4) gene, was extensively discussed in a context of association with anxiety, depression and other psychiatric conditions (Homberg and Lesch, 2011; Murphy et al., 2008). In our study, behavioural consequences of a 3-week Western diet feeding in aging female mice were exacerbated by knockout of the Sert gene (Veniaminova et al., under revision) (Chapter 4). Thus, Western diet-fed mice with complete Sert knockout (Sert-/-) demonstrated increased compared to wild type mice (WT) suppression in novelty exploration and hippocampus-dependent cognitive performance. Intensified effects of other environmental factors were shown in Sert-/- mice. For example, after mild postnatal foot-shock stress experiences or exposure to predator odors, anxiety-like behaviours were increased in Sert-/- but not WT mice (Adamec et al., 2006; Carroll et al., 2007). In response to chronic mild stress, Sert-/mice exhibited elevated plasma corticosterone levels (Lanfumey et al., 2000), and adrenocorticotropic hormone elevations were found in response to the placement of Sert-/- mice on the elevated plus maze (Li, 2006).

We found elevated Tlr 4 and decreased Ppargc1b expression in the hypothalamus of naïve Sert-/- mice (Veniaminova et al., submitted), suggesting the presence of proinflammatory status at the baseline in these mice which can contribute to their susceptibility to 
environmental factors. Further, we demonstrated Western diet-induced increase in Tlr4 expression in dorsal raphe and prefrontal cortex of Sert-/- mice, while in WT mice it was present only in dorsal raphe region (Veniaminova et al., submitted). In line with our findings, rats with a genetic decrease of Sert showed altered brain cytokine expression, exacerbated cytokine response to the LPS challenge and microglia activation (Macchi et al., 2013).

Naïve Sert-/- mice compared to WT displayed increased brain expression of Ppargc1a, serotonin receptors Htr1b and Htr2c (Veniaminova et al., submitted) and insulin receptor isoforms IRA and IRB (Veniaminova et al., unpublished; Fig. 5.3). These changes were "reversed" in animals fed with the Western diet. Changes in the expression of serotonin receptors were previously reported for Sert-/mice and discussed as being adaptive due to the compensatory rewiring of brain circuits and neurotransmitter systems in the life-long and developmental absence of Sert (Murphy et al., 2008). Decreased expression levels of Ppargc1a, Htr1b, Htr2c, IRA and IRB compared to naïve Sert-/- mice are associated with inflammation, impairment of metabolism and insulin receptor-mediated signaling (Handschin and Spiegelman, 2008; Nonogaki et al., 2006; Pomytkin et al., 2018).

Enhanced intestinal permeability and LPS leakage were shown in Sert-/- mice (El Aidy et al., 2017), which may have additive effect with diet-induced increases in gut permeability (see section 1.2) and 
inflammation. In addition, we demonstrated increased body weight and impaired glucose tolerance in Sert-/- mice housed on the Western diet (Veniaminova et al., submitted). These peripheral metabolic conditions might also contribute to neuroinflammation (discussed in section 5.4) resulting in aggravation of behavioural abnormalities seen in Sert-/mice.

Remarkably, unlike WT and SERT-/- mice, those heterozygous for the Sert gene (Sert+/-) did not show impairment in glucose tolerance, elevated brain expression of Tlr4, or disrupted hippocampusdependent performance (Veniaminova et al., submitted). While Sert+/- as well as WT and Sert-/- demonstrated increased Western diet-induced body weight gain and depressive-like behaviour, Sert+/mice exhibited partial resilience to the diet with regard to metabolic parameters.

While in the majority of reports, Sert $+/$ - rodents demonstrated an intermediate phenotype between WT and Sert-/- (Murphy et al., 2008), some findings suggest distinct physiology of the two Sertdeficient genotypes. Sert+/- differed from WT and Sert-/- by expression of HPA regulatory protein binding protein 5 (FK506) in the pituitary of mice exposed to early life stress model (van der Doelen et al., 2014) and reduced basal corticosterone plasma levels (Houwing et al., 2017). However, in the other studies, Sert+/- animals demonstrate 
higher than WT or even Sert-/- vulnerability to environmental stimuli (Macchi et al., 2013; van den Hove et al., 2011).

It is also observed in clinical and animal studies that heterozygosity of Sert or s allele may lead to a beneficial effect on cognition (Fiedorowicz et al., 2007; Homberg and Lesch, 2011; van den Hove et al., 2011). In this case it is important to highlight again that in our study in Sert+/- mice, unlike WT and Sert-/-, Western diet did not impair performance in the test for hippocampus-dependent performance (Veniaminova et al., under revision) (Chapter 4).

Together, based on the data discussed in the sections 5.1-5.5 and our results presented in chapters $2-4$, it can be proposed that Western diet may induce neuroinflammation, oxidative stress and mitochondrial dysfunction mediated both by peripheral inflammation and direct interaction between diet components and brain molecular pathways related to TLR4 and PPARs, which interact with functioning of serotonergic system and activity of insulin receptors. The molecular brain changes are associated with depression, and generate in mice ASD-like and ADHD-like behaviours that could lead to aggravation of the symptoms if replicated in man (Fig. 5.4). 


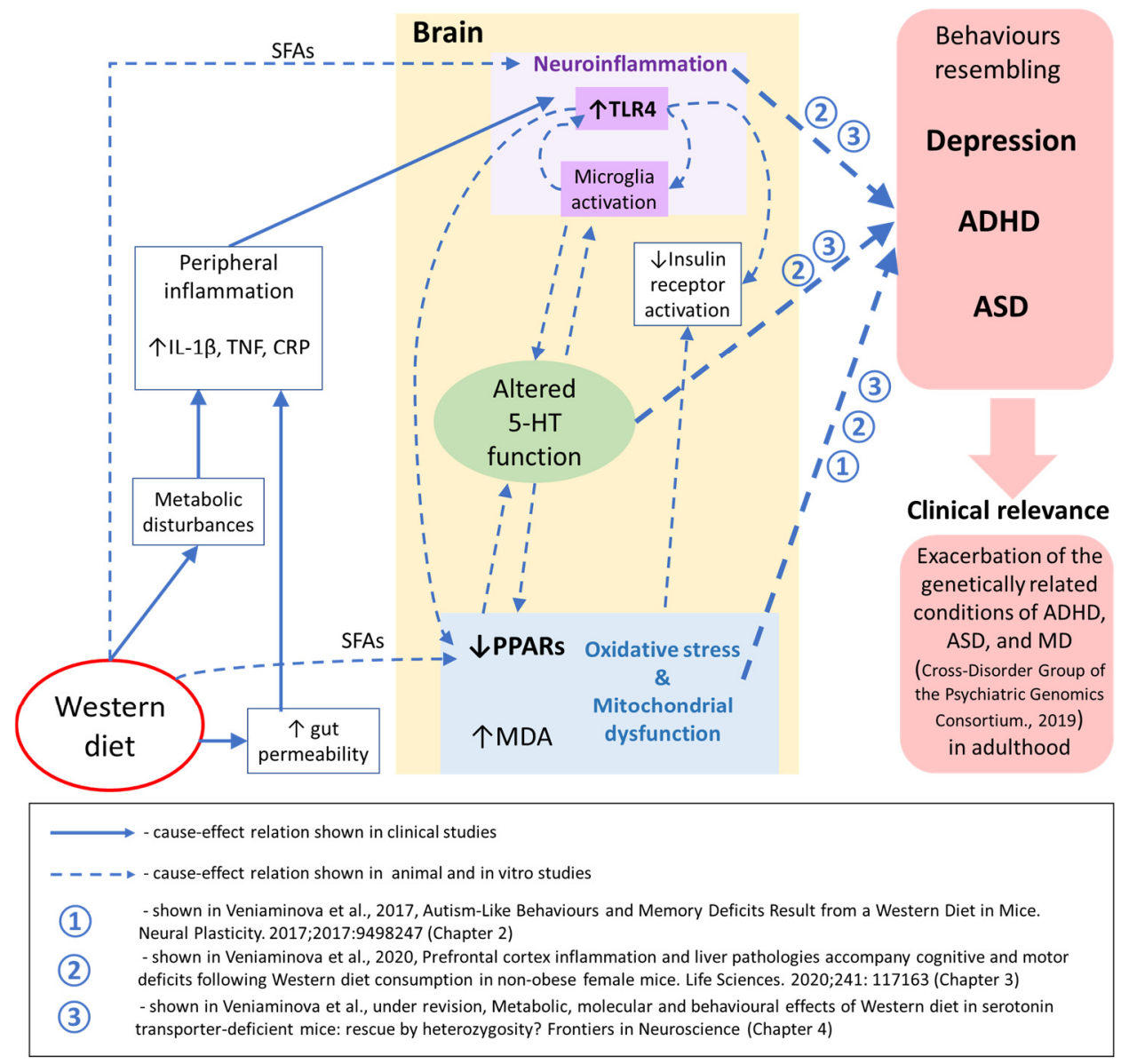

Figure 5.4. Proposed mechanism of the Western diet-induced aggravation of depression, ADHD and ASD symptoms. The effects of the Western diet effects are mediated both by peripheral inflammation and the associated systemic metabolic changes, increased gut permeability, as well as a direct interaction between diet components and brain molecular pathways related to TLR4 and PPARs. This leads to neuroinflammation, oxidative stress and mitochondrial dysfunction, altered function of the serotonergic system and decreased activity of insulin receptors. The molecular brain changes are associated with depression, and generate ASD-like and ADHD-like behaviours that could lead to aggravation of the symptoms if replicated in man. SFAs - saturated fatty acids, IL-1 $\beta$ - interleukin $1 \beta$, TNF - tumor necrosis factor, CRP - C-reactive protein, MDA - malondialdehyde, 5-HT - serotonin, TLR4 - toll-like receptor 4, PPARs - peroxisome proliferator-activated receptors, ASD - autism spectrum disorder, ADHD - attention deficit/hyperactivity disorder, MD - major depression 


\subsection{Limitations of animal models of Western diet and Sert deficiency when studying human pathologies}

Rodents are widely used model organisms in human disease research. Mouse and rat models have been used extensively to provide insight into the mechanisms underlying many diseases, to explore the efficacy of candidate drugs, and to predict patient response to treatment. Development of methods for the creation of transgenic, knockout and knockin mice have provided powerful tools for research and have made mice the most commonly used model organism (Rosenthal and Brown, 2007). However, the use of mice in biomedical research must take into consideration both the differences and similarities between mice and humans.

First, given that mice have a greater surface area to volume ratio than humans, their metabolic rate per gram of tissue is roughly seven times higher (Kleiber, 1961). Differences in metabolic rate between mice and humans are correlated with many anatomic, physiologic and biochemical differences. Mice have relatively larger metabolically active tissues, such as the liver and kidney, and larger deposits of brown fat. Mouse cells differ from human cells not only in mitochondrial density and metabolic rate, but also in the fatty acid composition of their membrane phospholipids (Hulbert, 2008). Mice have higher rates of production of reactive oxygen species and suffer higher rates of oxidative damage than do humans. 
Importantly to nutrition research, mice have different microbiomes (Nguyen et al., 2015) and have coevolved with different pathogens than humans. The anatomy of the gastrointestinal track differs between the two species, as mice have a prominent cecum and no appendix (Nguyen et al., 2015). Thus, the two species provide different environments that apparently support the growth of different gastrointestinal microbiota. Despite this fact, the trend of microbiota shift due to Western diet consumption observed in mouse studies agrees with that found in human studies (Nguyen et al., 2015). While main features of the immune system are similar in mice and humans, there are also significant differences due to coevolution with different pathogens and the microbiome (Bailey et al., 2013). Moreover, mice exhibit differences in lipid metabolism versus humans. For example, they lack cholesteryl ester transfer protein (Guyard-Dangremont et al., 1998) which shuttles cholesteryl esters from high density lipoproteins (HDL) to low (LDL) and very low density lipoproteins in humans. Thus, mice carry the majority of plasma cholesterol in HDL while humans carry much of it in LDL (Camus et al., 1983).

Due to discussed differences in metabolism and the immune system, dietary effects will not be the same in mouse and human. Moreover, a large number of diets used for studies in rodents may not appropriately represent the general human diet, particularly the Western diet. Typical Western diet in human on average consists of 
30-40\% dietary fat (Ford and Dietz, 2013). Therefore, using, for example, $60 \%$ fat in rodent dietary interventions is too extreme compared with the average Western diet. In our study we used the diet which as closely as possible mimics fat percentage of human Western diet and contains around $40 \%$ of calories from fat. Human Western diet can be also associated with decreased intake of vitamins, lack of certain nutrients and excessive alcohol and sodium (Koliaki and Katsilambros, 2013), which are not found in standard rodent Western diet feed and which effects are investigated distinctly in separate studies. In addition, although both mice and humans are omnivores, standard control diet in rodents is mainly grain based and differs significantly to human diet (Perlman, 2016). While in humans dietinduced obesity and associated disease occurs over months, years, and decades, it takes only several weeks of Western diet feeding to induce obesity in mouse models, making it impossible to translate the relevance of duration of metabolic disease development to humans (Lai et al., 2014). For example, in our study, 3-week Western diet induced impaired glucose tolerance both in young and aging female mice, while weight gain was found only in aging animals (Veniaminova et al., 2017, 2020, under revision) (Chapters 2-4). Because of the rapid disease induction, mouse models often lack vascular complications associated with metabolic disorders (Wu et al., 2006). 
Nevertheless, using the Western diet model in mice provides an opportunity to reproduce general features of metabolic disorders and further investigate molecular mechanisms of their effects on the brain. Thus, in young female mice fed with the Western diet we demonstrated metabolic alterations as well as behavioural phenotypes reminiscent of ASD/ADHD-like symptoms and proposed that neuroinflammation and oxidative stress may undelay these changes (Veniaminova et al., 2017, 2020) (Chapters 2 and 3). While our results cannot be directly translated to a human context, they highlight the importance of future clinical studies in investigating the effects of dietary pattern in patients with ASD or ADHD and in groups of high risk for developing these disorders.

Manipulations of the mouse genome provide a tool to investigate the role of certain genes and to model the effects of a combination of environmental factors and allele gene variants on pathology. While mice do not have 5-HTTLPR polymorphism in the SERT gene found in human, Sert $+/-$ mouse closely resemble human 5 -HTTLPR ss genotype in regard to levels of SERT expression and associated functional consequences (Murphy et al., 2008). As it was reviewed previously, Sert-/- rodents, like s-allele carriers, demonstrate increased stress response in behavioural tests for anxiety-like behaviour (Kalueff et al., 2010). Several studies showed that Sert+/- mice develop corresponding behavioural changes when exposed to psychosocial stress 
in early life or adulthood (Bartolomucci et al., 2010; Jansen et al., 2010; Lewejohann et al., 2010), which is also associated with ss genotype. However, one study demonstrated signs of reduced anxiety and enhanced memory performance in Sert+/- compared to WT (van den Hove et al., 2011). Depressive-like behaviour however is less consistent for Sert-/- rodents and highly dependent on genetic background (Kalueff et al., 2010) which suggests an interaction between the genes, a factor that is not translatable to human variability. Consistent with humans, however, improved cognitive performance was reported in Sert-/- rodents (Brigman et al., 2010). In summary, some of the traits of the ss genotype are more closely associated with mouse Sert+/genotype, while others with Sert-/- genotype.

While the s allele in human was associated with obesity and type 2 diabetes (Fuemmeler et al., 2008; Iordanidou et al., 2010; Sookoian et al., 2007), no clinical studies investigated the susceptibility of s allele carriers to excessive intake of fat and sugar. In our study, some behavioural, metabolic and molecular changes induced in Sert-/- mice by the Western diet were not induced in Sert+/- (Veniaminova et al., under revision) (Chapter 4). Thus, opposite to Sert-/- and WT, Sert $+/-$ mice did not develop impairment in glucose tolerance after three weeks of Western diet feeding. Translation of this finding into human situation is limited, suggesting, however, that 5-HTTLPR polymorphism may play an important role in the susceptibility to the 
effects of the Western diet. Future studies in this mouse model with a longer period of dietary feeding, as well as retrospective studies in humans, will be important to clarify these findings.

\subsection{Future perspectives}

The next step for our project is to run additional studies on the brain tissue of mice with different Sert genotype fed either with the Western or control diet. First, high performance liquid chromatography (HPLC) analysis of 5-HT and 5-HIAA brain levels will show the diet-induced changes of tryptophan-serotonin metabolism. Then, the measurement of Sert level in the brain of Sert $+/$ - mice after Western diet feeding might provide a key to the mechanisms of their resilience to the effects of the diet. Further, the examination of metabolome of brain, liver and blood is essential for complex understanding of the difference in diet effects in mice with partial and complete Sert deficiency, which underly different behavioural and metabolic outcomes. Data is already obtained for the blood metabolome and the paper is in preparation.

As we hypothesized significant role of the TLR4 in the negative effects of the Western diet, the use of TLR4 antagonists in our model, in case of its protective effect, would support our hypothesis. It could be also suggested to perform a study with the Western diet feeding on mice, which were treated with SSRI starting from an early age, and 
see if it would mimic the situation of Sert+/- or Sert-/- mice in respect to their susceptibility to the Western diet.

In order to further expand our understanding of the interaction between Sert and the Western diet, studies with the same experimental protocol can be conducted on male mice, young mice with Sert deficiency, and longer period of dietary intervention can be applied.

\section{References}

Adamec, R., Burton, P., Blundell, J., Murphy, D., Holmes, A., 2006. Vulnerability to mild predator stress in serotonin transporter knockout mice. Behav. Brain Res. 170, 126-140. https://doi.org/10.1016/j.bbr.2006.02.012

Agarwal, S., Yadav, A., Chaturvedi, R.K., 2017. Peroxisome proliferatoractivated receptors (PPARs) as therapeutic target in neurodegenerative disorders. Biochem. Biophys. Res. Commun. 483, 1166-1177. https://doi.org/10.1016/j.bbrc.2016.08.043

Ahmad, S.F., Ansari, M.A., Nadeem, A., Alzahrani, M.Z., Bakheet, S.A., Attia, S.M., 2018. Resveratrol Improves Neuroimmune Dysregulation Through the Inhibition of Neuronal Toll-Like Receptors and COX-2 Signaling in BTBR $\mathrm{T}+$ Itpr3tf/J Mice. NeuroMolecular Med. 20, 133-146. https://doi.org/10.1007/s12017-018-8483-0

Aleshin, S., Reiser, G., 2013. Role of the peroxisome proliferator-activated receptors (PPAR) $-\alpha, \beta / \delta$ and $\gamma$ triad in regulation of reactive oxygen species signaling in brain. Biol. Chem. 394, 1553-1570. https://doi.org/10.1515/hsz2013-0215

Alpak, G., Selek, S., Bulut, M., Bulbul, F., Unal, A., Virit, O., Kilic, O.H.T., Gergerlioglu, H.S., Savas, H.A., 2014. High Catalase and Low Thiol Levels in Adult-ADHD Patients. Klin. Psikofarmakol. Bülteni-Bulletin Clin. Psychopharmacol. 24, 128-134. https://doi.org/10.5455/bcp.20140429091127

Anand, D., Colpo, G.D., Zeni, G., Zeni, C.P., Teixeira, A.L., 2017. Attention-Deficit/Hyperactivity Disorder And Inflammation: What Does Current Knowledge Tell Us? A Systematic Review. Front. psychiatry 8, 228. https://doi.org/10.3389/fpsyt.2017.00228

Antonietta Ajmone-Cat, M., Lavinia Salvatori, M., De Simone, R., 
Mancini, M., Biagioni, S., Bernardo, A., Cacci, E., Minghetti, L., 2012. Docosahexaenoic acid modulates inflammatory and antineurogenic functions of activated microglial cells. J. Neurosci. Res. 90, 575-587. https://doi.org/10.1002/jnr.22783

Appel, S., Mirakaj, V., Bringmann, A., Weck, M.M., Grünebach, F., Brossart, P., 2005. PPAR- $\gamma$ agonists inhibit toll-like receptor-mediated activation of dendritic cells via the MAP kinase and NF- $x$ B pathways. Blood 106, 3888-3894. https://doi.org/10.1182/blood-2004-12-4709

Avcil, S., Uysal, P., Avcil, M., Alışık, M., Biçer, C., 2017. Dynamic thiol/disulfide homeostasis in children with attention deficit hyperactivity disorder and its relation with disease subtypes. Compr. Psychiatry 73, 53-60. https://doi.org/10.1016/j.comppsych.2016.11.003

Bahniwal, M., Little, J.P., Klegeris, A., 2017. High Glucose Enhances Neurotoxicity and Inflammatory Cytokine Secretion by Stimulated Human Astrocytes. Curr. Alzheimer

Res.

14 . https://doi.org/10.2174/1567205014666170117104053

Bailey, M., Christoforidou, Z., Lewis, M.C., 2013. The evolutionary basis for differences between the immune systems of man, mouse, pig and ruminants. Vet. Immunol. Immunopathol. 152, 13-19. https://doi.org/10.1016/j.vetimm.2012.09.022

Bartolomucci, A., Carola, V., Pascucci, T., Puglisi-Allegra, S., Cabib, S., Lesch, K.-P., Parmigiani, S., Palanza, P., Gross, C., 2010. Increased vulnerability to psychosocial stress in heterozygous serotonin transporter knockout mice. Dis. Model. Mech. 3, 459-470. https://doi.org/10.1242/dmm.004614

Beasley, C.L., Pennington, K., Behan, A., Wait, R., Dunn, M.J., Cotter, D., 2006. Proteomic analysis of the anterior cingulate cortex in the major psychiatric disorders: Evidence for disease-associated changes. Proteomics 6, 3414-3425. https://doi.org/10.1002/pmic.200500069

Ben-Shachar, D., Karry, R., 2008. Neuroanatomical Pattern of Mitochondrial Complex I Pathology Varies between Schizophrenia, Bipolar Disorder and Major Depression. PLoS One 3, e3676. https://doi.org/10.1371/journal.pone.0003676

Bennett, F.C., Molofsky, A. V., 2019. The immune system and psychiatric disease: a basic science perspective. Clin. Exp. Immunol. cei.13334. https://doi.org/10.1111/cei.13334

Brigman, J.L., Mathur, P., Harvey-White, J., Izquierdo, A., Saksida, L.M., Bussey, T.J., Fox, S., Deneris, E., Murphy, D.L., Holmes, A., 2010. 
Pharmacological or Genetic Inactivation of the Serotonin Transporter Improves Reversal Learning in Mice. Cereb. Cortex 20, 1955-1963. https://doi.org/10.1093/cercor/bhp266

Buffington, S.A., Di Prisco, G.V., Auchtung, T.A., Ajami, N.J., Petrosino, J.F., Costa-Mattioli, M., 2016. Microbial Reconstitution Reverses Maternal Diet-Induced Social and Synaptic Deficits in Offspring. Cell 165, 1762-1775. https://doi.org/10.1016/j.cell.2016.06.001

Bulut, M., Selek, S., Gergerlioglu, H.S., Savas, H.A., Yilmaz, H.R., Yuce, M., Ekici, G., 2007. Malondialdehyde levels in adult attention-deficit hyperactivity disorder. J. Psychiatry Neurosci. 32, 435-8.

Caesar, R., Tremaroli, V., Kovatcheva-Datchary, P., Cani, P.D., Bäckhed, F., 2015. Crosstalk between Gut Microbiota and Dietary Lipids Aggravates WAT Inflammation through TLR Signaling. Cell Metab. 22, 658-68. https://doi.org/10.1016/j.cmet.2015.07.026

Camus, M.C., Chapman, M.J., Forgez, P., Laplaud, P.M., 1983. Distribution and characterization of the serum lipoproteins and apoproteins in the mouse, Mus musculus. J. Lipid Res. 24, 1210-28.

Carpenter Rich, E., Loo, S.K., Yang, M., Dang, J., Smalley, S.L., 2009. Social functioning difficulties in ADHD: association with PDD risk. Clin. Child Psychol. Psychiatry 14, 329-44. https://doi.org/10.1177/1359104508100890

Carroll, J.C., Boyce-Rustay, J.M., Millstein, R., Yang, R., Wiedholz, L.M., Murphy, D.L., Holmes, A., 2007. Effects of mild early life stress on abnormal emotion-related behaviors in 5-HTT knockout mice. Behav. Genet. 37, 21422. https://doi.org/10.1007/s10519-006-9129-9

Ceylan, M., Sener, S., Bayraktar, A.C., Kavutcu, M., 2010. Oxidative imbalance in child and adolescent patients with attentiondeficit/hyperactivity disorder. Prog. Neuropsychopharmacol. Biol. Psychiatry 34, 1491-4. https://doi.org/10.1016/j.pnpbp.2010.08.010

Ceylan, M.F., Sener, S., Bayraktar, A.C., Kavutcu, M., 2012. Changes in oxidative stress and cellular immunity serum markers in attentiondeficit/hyperactivity disorder. Psychiatry Clin. Neurosci. 66, 220-226. https://doi.org/10.1111/j.1440-1819.2012.02330.x

Chauhan, A., Chauhan, V., Brown, W.T., Cohen, I., 2004. Oxidative stress in autism: Increased lipid peroxidation and reduced serum levels of ceruloplasmin and transferrin - the antioxidant proteins. Life Sci. 75, 25392549. https://doi.org/10.1016/j.lfs.2004.04.038

Chauhan, A., Gu, F., Essa, M.M., Wegiel, J., Kaur, K., Brown, W.T., 
Chauhan, V., 2011. Brain region-specific deficit in mitochondrial electron transport chain complexes in children with autism. J. Neurochem. 117, 209220. https://doi.org/10.1111/j.1471-4159.2011.07189.x

Chen, C.T., Kitson, A.P., Hopperton, K.E., Domenichiello, A.F., Trépanier, M.-O., Lin, L.E., Ermini, L., Post, M., Thies, F., Bazinet, R.P., 2015. Plasma non-esterified docosahexaenoic acid is the major pool supplying the brain. Sci. Rep. 5, 15791. https://doi.org/10.1038/srep15791

Chen, S., Zhang, H., Pu, H., Wang, G., Li, W., Leak, R.K., Chen, J., Liou, A.K., Hu, X., 2015. n-3 PUFA supplementation benefits microglial responses to myelin pathology. Sci. Rep. 4, 7458. https://doi.org/10.1038/srep07458

Chistyakov, D. V., Aleshin, S., Sergeeva, M.G., Reiser, G., 2014. Regulation of peroxisome proliferator-activated receptor $\beta / \delta$ expression and activity levels by toll-like receptor agonists and MAP kinase inhibitors in rat astrocytes. J. Neurochem. 130, 563-574. https://doi.org/10.1111/jnc.12757

Chistyakov, D. V., Aleshin, S.E., Astakhova, A.A., Sergeeva, M.G., Reiser, G., 2015. Regulation of peroxisome proliferator-activated receptors (PPAR) $\alpha$ and $-\gamma$ of rat brain astrocytes in the course of activation by toll-like receptor agonists. J. Neurochem. 134, 113-124. https://doi.org/10.1111/jnc.13101

Chovanová, Z., Muchová, J., Sivoňová, M., Dvořáková, M., Žitňanová, I., Waczulíková, I., Trebatická, J., Škodáček, I., Duračková, Z., 2006. Effect of polyphenolic extract, Pycnogenol @ , on the level of 8-oxoguanine in children suffering from attention deficit/hyperactivity disorder. Free Radic. Res. 40, 1003-1010. https://doi.org/10.1080/10715760600824902

Cipriani, C., Ricceri, L., Matteucci, C., De Felice, A., Tartaglione, A.M., Argaw-Denboba, A., Pica, F., Grelli, S., Calamandrei, G., Sinibaldi Vallebona, P., Balestrieri, E., 2018. High expression of Endogenous Retroviruses from intrauterine life to adulthood in two mouse models of Autism Spectrum Disorders. Sci. Rep. 8, 629. https://doi.org/10.1038/s41598-017-19035-w

Colle, R., de Larminat, D., Rotenberg, S., Hozer, F., Hardy, P., Verstuyft, C., Fève, B., Corruble, E., 2016. PPAR- $\gamma$ Agonists for the Treatment of Major Depression: A Review. Pharmacopsychiatry 50, 49-55. https://doi.org/10.1055/s-0042-120120

Creely, S.J., McTernan, P.G., Kusminski, C.M., Fisher, ff. M., Da Silva, N.F., Khanolkar, M., Evans, M., Harte, A.L., Kumar, S., 2007. Lipopolysaccharide activates an innate immune system response in human adipose tissue in obesity and type 2 diabetes. Am. J. Physiol. Metab. 292, E740-E747. https://doi.org/10.1152/ajpendo.00302.2006 
Cross-Disorder Group of the Psychiatric Genomics Consortium, 2019. Genomic Relationships, Novel Loci, and Pleiotropic Mechanisms across Eight Psychiatric Disorders. Cell 179, 1469-1482.e11.

https://doi.org/10.1016/j.cell.2019.11.020

D'Agostino, G., Cristiano, C., Lyons, D.J., Citraro, R., Russo, E., Avagliano, C., Russo, R., Raso, G.M., Meli, R., De Sarro, G., Heisler, L.K., Calignano, A., 2015. Peroxisome proliferator-activated receptor alpha plays a crucial role in behavioral repetition and cognitive flexibility in mice. Mol. Metab. 4, 528-36. https://doi.org/10.1016/j.molmet.2015.04.005

D'Eufemia, P., Celli, M., Finocchiaro, R., Pacifico, L., Viozzi, L., Zaccagnini, M., Cardi, E., Giardini, O., 1996. Abnormal intestinal permeability in children with autism. Acta Paediatr. 85, 1076-1079. https://doi.org/10.1111/j.1651-2227.1996.tb14220.x

Dana, N., Vaseghi, G., Haghjooy Javanmard, S., 2019. Crosstalk between Peroxisome Proliferator-Activated Receptors and Toll-Like Receptors: A Systematic Review. Adv. Pharm. Bull. 9, 12-21. https://doi.org/10.15171/apb.2019.003

de Velasco, P., Ferreira, A., Crovesy, L., Marine, T., das Graças Tavares do Carmo, M., 2018. Fatty Acids, Gut Microbiota, and the Genesis of Obesity, in: Biochemistry and Health Benefits of Fatty Acids. IntechOpen. https://doi.org/10.5772/intechopen.80664

Delerive, P., Fruchart, J.C., Staels, B., 2001. Peroxisome proliferatoractivated receptors in inflammation control. J. Endocrinol. 169, 453-9. https://doi.org/10.1677/joe.0.1690453

Deyama, S., Ishikawa, Y., Yoshikawa, K., Shimoda, K., Ide, S., Satoh, M., Minami, M., 2017. Resolvin D1 and D2 Reverse Lipopolysaccharide-Induced Depression-Like Behaviors Through the mTORC1 Signaling Pathway. Int. J. Neuropsychopharmacol. 20, 575-584. https://doi.org/10.1093/ijnp/pyx023

Ding, S., Chi, M.M., Scull, B.P., Rigby, R., Schwerbrock, N.M.J., Magness, S., Jobin, C., Lund, P.K., 2010. High-fat diet: bacteria interactions promote intestinal inflammation which precedes and correlates with obesity and insulin resistance in mouse. PLoS One 5, e12191. https://doi.org/10.1371/journal.pone.0012191

El Aidy, S., Ramsteijn, A.S., Dini-Andreote, F., van Eijk, R., Houwing, D.J., Salles, J.F., Olivier, J.D.A., 2017. Serotonin Transporter Genotype Modulates the Gut Microbiota Composition in Young Rats, an Effect Augmented by Early Life Stress. Front. Cell. Neurosci. 11, 222. https://doi.org/10.3389/fncel.2017.00222 
Ende, G., Demirakca, T., Walter, S., Wokrina, T., Sartorius, A., Wildgruber, D., Henn, F.A., 2007. Subcortical and medial temporal MRdetectable metabolite abnormalities in unipolar major depression. Eur. Arch. Psychiatry Clin. Neurosci. 257, 36-39. https://doi.org/10.1007/s00406-0060680-4

Enstrom, A.M., Onore, C.E., Van de Water, J.A., Ashwood, P., 2010. Differential monocyte responses to TLR ligands in children with autism spectrum disorders. Brain. Behav. Immun. 24, 64-71. https://doi.org/10.1016/j.bbi.2009.08.001

Felger, J.C., Lotrich, F.E., 2013. Inflammatory cytokines in depression: neurobiological mechanisms and therapeutic implications. Neuroscience 246, 199-229. https://doi.org/10.1016/j.neuroscience.2013.04.060

Femenia, T., Qian, Y., Arentsen, T., Forssberg, H., Diaz Heijtz, R., 2018. Toll-like receptor-4 regulates anxiety-like behavior and DARPP-32 phosphorylation. Brain. Behav. Immun. 69, 273-282. https://doi.org/10.1016/j.bbi.2017.11.022

Ferreira, A.E., Sisti, F., Sônego, F., Wang, S., Filgueiras, L.R., Brandt, S., Serezani, A.P.M., Du, H., Cunha, F.Q., Alves-Filho, J.C., Serezani, C.H., 2014. PPAR- $\gamma /$ IL-10 axis inhibits MyD88 expression and ameliorates murine polymicrobial sepsis. J. Immunol. 192, 2357-65. https://doi.org/10.4049/jimmunol.1302375

Fiedorowicz, J.G., Moser, D.J., Hynes, S.M., Beglinger, L.J., Schultz, S.K., Ellingrod, V.L., 2007. LA allelic heterozygosity of the 5HTTLPR polymorphism is associated with higher cognitive function and lower interpersonal sensitivity. Psychiatr. Genet. 17, 3-4. https://doi.org/10.1097/YPG.0b013e328010f498

Fliers, E., Rommelse, N., Vermeulen, S.H.H.M., Altink, M., Buschgens, C.J.M., Faraone, S. V., Sergeant, J.A., Franke, B., Buitelaar, J.K., 2008. Motor coordination problems in children and adolescents with ADHD rated by parents and teachers: effects of age and gender. J. Neural Transm. 115, 211-220. https://doi.org/10.1007/s00702-007-0827-0

Ford, E.S., Dietz, W.H., 2013. Modeling dietary patterns to assess sodium recommendations for nutrient adequacy. Am. J. Clin. Nutr. 97, 848-853. https://doi.org/10.3945/ajcn.112.052662

Fournier, K.A., Hass, C.J., Naik, S.K., Lodha, N., Cauraugh, J.H., 2010. Motor Coordination in Autism Spectrum Disorders: A Synthesis and MetaAnalysis. J. Autism Dev. Disord. 40, 1227-1240. https://doi.org/10.1007/s10803-010-0981-3 
Fourrier, C., Remus-Borel, J., Greenhalgh, A.D., Guichardant, M., Bernoud-Hubac, N., Lagarde, M., Joffre, C., Layé, S., 2017. Docosahexaenoic acid-containing choline phospholipid modulates LPS-induced neuroinflammation in vivo and in microglia in vitro. J. Neuroinflammation 14, 170. https://doi.org/10.1186/s12974-017-0939-x

Fredericks, C.A., Drabant, E.M., Edge, M.D., Tillie, J.M., Hallmayer, J., Ramel, W., Kuo, J.R., Mackey, S., Gross, J.J., Dhabhar, F.S., 2010. Healthy young women with serotonin transporter SS polymorphism show a proinflammatory bias under resting and stress conditions. Brain. Behav. Immun. 24, 350-357. https://doi.org/10.1016/j.bbi.2009.10.014

Freund-Levi, Y., Eriksdotter-Jönhagen, M., Cederholm, T., Basun, H., Faxén-Irving, G., Garlind, A., Vedin, I., Vessby, B., Wahlund, L.-O., Palmblad, J., 2006. $\omega-3$ Fatty Acid Treatment in 174 Patients With Mild to Moderate Alzheimer Disease: OmegAD Study. Arch. Neurol. 63, 1402. https://doi.org/10.1001/archneur.63.10.1402

Froehlich, T.E., Anixt, J.S., Loe, I.M., Chirdkiatgumchai, V., Kuan, L., Gilman, R.C., 2011. Update on environmental risk factors for attentiondeficit/hyperactivity disorder. Curr. Psychiatry Rep. 13, 333-44. https://doi.org/10.1007/s11920-011-0221-3

Fuemmeler, B.F., Agurs-Collins, T.D., Mcclernon, F.J., Kollins, S.H., Kail, M.E., Bergen, A.W., Ashley-Koch, A.E., 2008. Genes Implicated in Serotonergic and Dopaminergic Functioning Predict BMI Categories. Obesity 16, 348-355. https://doi.org/10.1038/oby.2007.65

Gałecki, P., Szemraj, J., Bieńkiewicz, M., Florkowski, A., Gałecka, E., 2009. Lipid peroxidation and antioxidant protection in patients during acute depressive episodes and in remission after fluoxetine treatment. Pharmacol. Reports 61, 436-447. https://doi.org/10.1016/S1734-1140(09)70084-2

Gardner, A., 2003. Alterations of mitochondrial function and correlations with personality traits in selected major depressive disorder patients. J. Affect. Disord. 76, 55-68. https://doi.org/10.1016/S0165-0327(02)00067-8

Ghaleiha, A., Rasa, S.M., Nikoo, M., Farokhnia, M., Mohammadi, M.-R., Akhondzadeh, S., 2015. A pilot double-blind placebo-controlled trial of pioglitazone as adjunctive treatment to risperidone: Effects on aberrant behavior in children with autism. Psychiatry Res. 229, 181-187. https://doi.org/10.1016/j.psychres.2015.07.043

Gray, S.M., Aylor, K.W., Barrett, E.J., 2017. Unravelling the regulation of insulin transport across the brain endothelial cell. Diabetologia 60, 15121521. https://doi.org/10.1007/s00125-017-4285-4 
Greenberg, A.S., Obin, M.S., 2006. Obesity and the role of adipose tissue in inflammation and metabolism. Am. J. Clin. Nutr. 83, 461S-465S. https://doi.org/10.1093/ajcn/83.2.461S

Gu, F., Chauhan, V., Kaur, K., Brown, W.T., LaFauci, G., Wegiel, J., Chauhan, A., 2013. Alterations in mitochondrial DNA copy number and the activities of electron transport chain complexes and pyruvate dehydrogenase in the frontal cortex from subjects with autism. Transl. Psychiatry 3, e299e299. https://doi.org/10.1038/tp.2013.68

Guevara-Campos, J., González-Guevara, L., Briones, P., López-Gallardo, E., Bulán, N., Ruiz-Pesini, E., Ramnarine, D., Montoya, J., 2010. Autism associated to a deficiency of complexes III and IV of the mitochondrial respiratory chain. Invest. Clin. 51, 423-31.

Guney, E., Cetin, F.H., Alisik, M., Tunca, H., Tas Torun, Y., Iseri, E., Isik Taner, Y., Cayci, B., Erel, O., 2015. Attention Deficit Hyperactivity Disorder and oxidative stress: A short term follow up study. Psychiatry Res. 229, 310-317. https://doi.org/10.1016/j.psychres.2015.07.003

Gurley, C., Nichols, J., Liu, S., Phulwani, N.K., Esen, N., Kielian, T., 2008. Microglia and Astrocyte Activation by Toll-Like Receptor Ligands: Modulation by PPAR- $<$ math $><$ mi $>\gamma</$ mi $></$ math $>$ Agonists. PPAR Res. 2008, 1-15. https://doi.org/10.1155/2008/453120

Guyard-Dangremont, V., Desrumaux, C., Gambert, P., Lallemant, C., Lagrost, L., 1998. Phospholipid and cholesteryl ester transfer activities in plasma from 14 vertebrate species. Relation to atherogenesis susceptibility. Comp. Biochem. Physiol. Part B Biochem. Mol. Biol. 120, 517-525. https://doi.org/10.1016/S0305-0491(98)10038-X

Hachem, M., Géloën, A., Van, A. Lo, Foumaux, B., Fenart, L., Gosselet, F., Da Silva, P., Breton, G., Lagarde, M., Picq, M., Bernoud-Hubac, N., 2016. Efficient Docosahexaenoic Acid Uptake by the Brain from a Structured Phospholipid. Mol. Neurobiol. 53, 3205-3215. https://doi.org/10.1007/s12035-015-9228-9

Handschin, C., Spiegelman, B.M., 2008. The role of exercise and PGC1alpha in inflammation and chronic disease. Nature 454, 463-9. https://doi.org/10.1038/nature07206

Hassan, A.M., Mancano, G., Kashofer, K., Fröhlich, E.E., Matak, A., Mayerhofer, R., Reichmann, F., Olivares, M., Neyrinck, A.M., Delzenne, N.M., Claus, S.P., Holzer, P., 2019. High-fat diet induces depression-like behaviour in mice associated with changes in microbiome, neuropeptide Y, and brain metabolome. Nutr. Neurosci. 22, 877-893. 
https://doi.org/10.1080/1028415X.2018.1465713

Hasselbalch, S.G., Knudsen, G.M., Capaldo, B., Postiglione, A., Paulson, O.B., 2001. Blood-Brain Barrier Transport and Brain Metabolism of Glucose during Acute Hyperglycemia in Humans 1. J. Clin. Endocrinol. Metab. 86, 1986-1990. https://doi.org/10.1210/jcem.86.5.7490

Heilskov Rytter, M.J., Andersen, L.B.B., Houmann, T., Bilenberg, N., Hvolby, A., Mølgaard, C., Michaelsen, K.F., Lauritzen, L., 2015. Diet in the treatment of ADHD in children - a systematic review of the literature. Nord. J. Psychiatry 69, 1-18. https://doi.org/10.3109/08039488.2014.921933

Hernandez, E.D., Lee, S.J., Kim, J.Y., Duran, A., Linares, J.F., Yajima, T., Müller, T.D., Tschöp, M.H., Smith, S.R., Diaz-Meco, M.T., Moscat, J., 2014. A Macrophage NBR1-MEKK3 Complex Triggers JNK-Mediated Adipose Tissue Inflammation in Obesity. Cell Metab. 20, 499-511. https://doi.org/10.1016/j.cmet.2014.06.008

Hjorth, E., Zhu, M., Toro, V.C., Vedin, I., Palmblad, J., Cederholm, T., Freund-Levi, Y., Faxen-Irving, G., Wahlund, L.-O., Basun, H., Eriksdotter, M., Schultzberg, M., 2013. Omega-3 Fatty Acids Enhance Phagocytosis of Alzheimer's Disease-Related Amyloid- $\beta 42$ by Human Microglia and Decrease Inflammatory Markers. J. Alzheimer's Dis. 35, 697-713. https://doi.org/10.3233/JAD-130131

Hoekstra, J.H., van Kempen, A.A., Bijl, S.B., Kneepkens, C.M., 1993. Fructose breath hydrogen tests. Arch. Dis. Child. 68, 136-138. https://doi.org/10.1136/adc.68.1.136

Holmes, S.E., Hinz, R., Conen, S., Gregory, C.J., Matthews, J.C., AntonRodriguez, J.M., Gerhard, A., Talbot, P.S., 2018. Elevated Translocator Protein in Anterior Cingulate in Major Depression and a Role for Inflammation in Suicidal Thinking: A Positron Emission Tomography Study. Biol. Psychiatry 83, 61-69. https://doi.org/10.1016/j.biopsych.2017.08.005

Holzer, R.G., Park, E.-J., Li, N., Tran, H., Chen, M., Choi, C., Solinas, G., Karin, M., 2011. Saturated Fatty Acids Induce c-Src Clustering within Membrane Subdomains, Leading to JNK Activation. Cell 147, 173-184. https://doi.org/10.1016/j.cell.2011.08.034

Homberg, J.R., Lesch, K.-P., 2011. Looking on the bright side of serotonin transporter gene variation. Biol. Psychiatry 69, 513-9. https://doi.org/10.1016/j.biopsych.2010.09.024

Houwing, D.J., Buwalda, B., van der Zee, E.A., de Boer, S.F., Olivier, J.D.A., 2017. The Serotonin Transporter and Early Life Stress: Translational Perspectives.

Front.

Cell.

Neurosci.

11 ,

117. 
https://doi.org/10.3389/fncel.2017.00117

Hsieh, C.-F., Liu, C.-K., Lee, C.-T., Yu, L.-E., Wang, J.-Y., 2019. Acute glucose fluctuation impacts microglial activity, leading to inflammatory $\begin{array}{lllll}\text { activation or } & \text { self-degradation. } & \text { Sci. } & \text { Rep. } & 940 .\end{array}$ https://doi.org/10.1038/s41598-018-37215-0

Hulbert, A.J., 2008. The links between membrane composition, metabolic rate and lifespan. Comp. Biochem. Physiol. Part A Mol. Integr. Physiol. 150, 196-203. https://doi.org/10.1016/j.cbpa.2006.05.014

Hwang, I.W., Hong, J.H., Kwon, B.N., Kim, H.J., Lee, N.R., Lim, M.H., Kwon, H.J., Jin, H.J., 2017. Association of mitochondrial DNA 10398 A/G polymorphism with attention deficit and hyperactivity disorder in Korean children. Gene 630, 8-12. https://doi.org/10.1016/j.gene.2017.08.004

Inoue, T., Tanaka, M., Masuda, S., Ohue-Kitano, R., Yamakage, H., Muranaka, K., Wada, H., Kusakabe, T., Shimatsu, A., Hasegawa, K., SatohAsahara, N., 2017. Omega-3 polyunsaturated fatty acids suppress the inflammatory responses of lipopolysaccharide-stimulated mouse microglia by activating SIRT1 pathways. Biochim. Biophys. Acta - Mol. Cell Biol. Lipids 1862, 552-560. https://doi.org/10.1016/j.bbalip.2017.02.010

Iordanidou, M., Tavridou, A., Petridis, I., Arvanitidis, K.I., Christakidis, D., Vargemezis, V., Manolopoulos, V.G., 2010. The serotonin transporter promoter polymorphism (5-HTTLPR) is associated with type 2 diabetes. Clin. Chim. Acta 411, 167-171. https://doi.org/10.1016/j.cca.2009.10.022

Jacob, R.J., Fan, X., Evans, M.L., Dziura, J., Sherwin, R.S., 2002. Brain glucose levels are elevated in chronically hyperglycemic diabetic rats: No evidence for protective adaptation by the blood brain barrier. Metabolism 51, 1522-1524. https://doi.org/10.1053/meta.2002.36347

James, S.J., Cutler, P., Melnyk, S., Jernigan, S., Janak, L., Gaylor, D.W., Neubrander, J.A., 2004. Metabolic biomarkers of increased oxidative stress and impaired methylation capacity in children with autism. Am. J. Clin. Nutr. 80, 1611-1617. https://doi.org/10.1093/ajcn/80.6.1611

Jansen, F., Heiming, R.S., Lewejohann, L., Touma, C., Palme, R., Schmitt, A., Lesch, K.P., Sachser, N., 2010. Modulation of behavioural profile and stress response by 5 -HTT genotype and social experience in adulthood. Behav. Brain Res. 207, 21-29. https://doi.org/10.1016/j.bbr.2009.09.033

Jia, H., Xu, S., Liu, Q., Liu, J., Xu, J., Li, W., Jin, Y., Ji, Q., 2016. Effect of pioglitazone on neuropathic pain and spinal expression of TLR-4 and cytokines. Exp. Ther. Med. 12, 2644-2650. https://doi.org/10.3892/etm.2016.3643 
Jialal, I., Huet, B.A., Kaur, H., Chien, A., Devaraj, S., 2012. Increased Toll-Like Receptor Activity in Patients With Metabolic Syndrome. Diabetes Care 35, 900-904. https://doi.org/10.2337/dc11-2375

Johnson, R.J., Rivard, C., Lanaspa, M.A., Otabachian-Smith, S., Ishimoto, T., Cicerchi, C., Cheeke, P.R., McIntosh, B., Hess, T., 2013. Fructokinase, Fructans, Intestinal Permeability, and Metabolic Syndrome: An Equine Connection? J. Equine Vet. Sci. 33, 120-126. https://doi.org/10.1016/j.jevs.2012.05.004

Jyonouchi, H., Geng, L., Cushing-Ruby, A., Quraishi, H., 2008. Impact of innate immunity in a subset of children with autism spectrum disorders: a case control study. J. Neuroinflammation 5, 52. https://doi.org/10.1186/1742-2094-5-52

Kalueff, A.V., Olivier, J.D.A., Nonkes, L.J.P., Homberg, J.R., 2010. Conserved role for the serotonin transporter gene in rat and mouse neurobehavioral endophenotypes. Neurosci. Biobehav. Rev. 34, 373-386. https://doi.org/10.1016/j.neubiorev.2009.08.003

Kannan, Jain, 2000. Oxidative stress and apoptosis. Pathophysiol. Off. J. Int. Soc. Pathophysiol. 7, 153-163. https://doi.org/10.1016/s09284680(00)00053-5

Kawatani, M., Tsukahara, H., Mayumi, M., 2011. Evaluation of oxidative stress status in children with pervasive developmental disorder and attention deficit hyperactivity disorder using urinary-specific biomarkers. Redox Rep. 16, 45-46. https://doi.org/10.1179/174329211X12968219310873

Kéri, S., Szabó, C., Kelemen, O., 2014. Expression of Toll-Like Receptors in peripheral blood mononuclear cells and response to cognitive-behavioral therapy in major depressive disorder. Brain. Behav. Immun. 40, 235-243. https://doi.org/10.1016/j.bbi.2014.03.020

Khanzode, S.D., Dakhale, G.N., Khanzode, S.S., Saoji, A., Palasodkar, R., 2003. Oxidative damage and major depression: the potential antioxidant action of selective serotonin re-uptake inhibitors. Redox Rep. 8, 365-370. https://doi.org/10.1179/135100003225003393

Kiecolt-Glaser, J.K., Belury, M.A., Andridge, R., Malarkey, W.B., Hwang, B.S., Glaser, R., 2012. Omega-3 supplementation lowers inflammation in healthy middle-aged and older adults: A randomized controlled trial. Brain. Behav. Immun. 26, 988-995. https://doi.org/10.1016/j.bbi.2012.05.011

Kielian, T., 2006. Toll-like receptors in central nervous system glial inflammation and homeostasis. J. Neurosci. Res. 83, 711-30. https://doi.org/10.1002/jnr.20767 
Kim, J.I., Lee, S.-Y., Park, M., Kim, S.Y., Kim, J.-W., Kim, S.A., Kim, B.-N., 2019. Peripheral Mitochondrial DNA Copy Number is Increased in Korean Attention-Deficit Hyperactivity Disorder Patients. Front. Psychiatry 10. https://doi.org/10.3389/fpsyt.2019.00506

Kim, J.J., Sears, D.D., 2010. TLR4 and Insulin Resistance. Gastroenterol. Res. Pract. 2010. https://doi.org/10.1155/2010/212563

Kleiber, M., 1961. The fire of life. An introduction to animal energetics. John Wiley \& Sons, Inc., New York: London.

Klein, J.A., Ackerman, S.L., 2003. Oxidative stress, cell cycle, and neurodegeneration. J. Clin. Invest. 111, 785-93. https://doi.org/10.1172/JCI18182

Kodydková, J., Vávrová, L., Zeman, M., Jirák, R., Macášek, J., Staňková, B., Tvrzická, E., Žák, A., 2009. Antioxidative enzymes and increased oxidative stress in depressive women. Clin. Biochem. 42, 1368-1374. https://doi.org/10.1016/j.clinbiochem.2009.06.006

Koliaki, C., Katsilambros, N., 2013. Dietary sodium, potassium, and alcohol: key players in the pathophysiology, prevention, and treatment of human hypertension. Nutr. Rev. 71, 402-411. https://doi.org/10.1111/nure.12036

Korte-Bouws, G., van Heesch, F., Westphal, K., Ankersmit, L., van Oosten, E., Güntürkün, O., Korte, S., 2018. Bacterial Lipopolysaccharide Increases Serotonin Metabolism in Both Medial Prefrontal Cortex and Nucleus Accumbens in Male Wild Type Rats, but Not in Serotonin Transporter Knockout Rats. Pharmaceuticals 11, 66. https://doi.org/10.3390/ph11030066

Kul, M., Unal, F., Kandemir, H., Sarkarati, B., Kilinc, K., Kandemir, S.B., 2015. Evaluation of Oxidative Metabolism in Child and Adolescent Patients with Attention Deficit Hyperactivity Disorder. Psychiatry Investig. 12, 3616. https://doi.org/10.4306/pi.2015.12.3.361

Lai, M., Chandrasekera, P.C., Barnard, N.D., 2014. You are what you eat, or are you? The challenges of translating high-fat-fed rodents to human obesity and diabetes. Nutr. Diabetes 4, e135-e135. https://doi.org/10.1038/nutd.2014.30

Lanfumey, L., Mannoury La Cour, C., Froger, N., Hamon, M., 2000. 5HT-HPA interactions in two models of transgenic mice relevant to major depression. Neurochem. Res. 25, 1199-206.

Latorre, E., Layunta, E., Grasa, L., Castro, M., Pardo, J., Gomollón, F., Alcalde, A.I., Mesonero, J.E., 2016. Intestinal Serotonin Transporter 
Inhibition by Toll-Like Receptor 2 Activation. A Feedback Modulation. PLoS One 11, e0169303. https://doi.org/10.1371/journal.pone.0169303

Lean, M.E.J., Te Morenga, L., 2016. Sugar and Type 2 diabetes. Br. Med. Bull. 120, 43-53. https://doi.org/10.1093/bmb/ldw037

Lecoultre, V., Egli, L., Carrel, G., Theytaz, F., Kreis, R., Schneiter, P., Boss, A., Zwygart, K., Lê, K.-A., Bortolotti, M., Boesch, C., Tappy, L., 2013. Effects of fructose and glucose overfeeding on hepatic insulin sensitivity and intrahepatic lipids in healthy humans. Obesity 21, 782-785. https://doi.org/10.1002/oby.20377

Lee, H.-M., Kim, J.-J., Kim, H.J., Shong, M., Ku, B.J., Jo, E.-K., 2013. Upregulated NLRP3 Inflammasome Activation in Patients With Type 2 Diabetes. Diabetes 62, 194-204. https://doi.org/10.2337/db12-0420

Leitner, Y., 2014. The co-occurrence of autism and attention deficit hyperactivity disorder in children - what do we know? Front. Hum. Neurosci. 8, 268. https://doi.org/10.3389/fnhum.2014.00268

Levine, J., Barak, Y., Chengappa, K.N.R., Rapoport, A., Rebey, M., Barak, V., 1999. Cerebrospinal Cytokine Levels in Patients with Acute Depression. Neuropsychobiology 40, 171-176. https://doi.org/10.1159/000026615

Lewejohann, L., Kloke, V., Heiming, R.S., Jansen, F., Kaiser, S., Schmitt, A., Lesch, K.P., Sachser, N., 2010. Social status and day-to-day behaviour of male serotonin transporter knockout mice. Behav. Brain Res. 211, 220-228. https://doi.org/10.1016/j.bbr.2010.03.035

Li, Q., 2006. Cellular and Molecular Alterations in Mice With Deficient and Reduced Serotonin Transporters. Mol. Neurobiol. 34, 51-66. https://doi.org/10.1385/MN:34:1:51

Lin, J., Handschin, C., Spiegelman, B.M., 2005. Metabolic control through the PGC-1 family of transcription coactivators. Cell Metab. 1, 361-370. https://doi.org/10.1016/j.cmet.2005.05.004

Lindqvist, D., Janelidze, S., Hagell, P., Erhardt, S., Samuelsson, M., Minthon, L., Hansson, O., Björkqvist, M., Träskman-Bendz, L., Brundin, L., 2009. Interleukin-6 is elevated in the cerebrospinal fluid of suicide attempters and related to symptom severity. Biol. Psychiatry 66, 287-92. https://doi.org/10.1016/j.biopsych.2009.01.030

Liu, K.-F., Li, Y., Cheng, K.C., Hsu, C.C., Cheng, J.-T., Peng, W.-H., 2017. Changes in PPAR $\delta$ expression in a rat model of stress-induced depression. Clin. Exp. Pharmacol. Physiol. 44, 664-670. https://doi.org/10.1111/1440-1681.12746 
Lukic, I., Mitic, M., Djordjevic, J., Tatalovic, N., Bozovic, N., Soldatovic, I., Mihaljevic, M., Pavlovic, Z., Radojcic, M.B., Maric, N.P., Adzic, M., 2014. Lymphocyte Levels of Redox-Sensitive Transcription Factors and Antioxidative Enzymes as Indicators of Pro-Oxidative State in Depressive Patients. Neuropsychobiology 70, 1-9. https://doi.org/10.1159/000362841

Ly, V., Bottelier, M., Hoekstra, P.J., Arias Vasquez, A., Buitelaar, J.K., Rommelse, N.N., 2017. Elimination diets' efficacy and mechanisms in attention deficit hyperactivity disorder and autism spectrum disorder. Eur. Child Adolesc. Psychiatry 26, 1067-1079. https://doi.org/10.1007/s00787017-0959-1

Lyall, K., Schmidt, R.J., Hertz-Picciotto, I., 2014. Maternal lifestyle and environmental risk factors for autism spectrum disorders. Int. J. Epidemiol. 43, 443-64. https://doi.org/10.1093/ije/dyt282

Macchi, F., Homberg, J.R., Calabrese, F., Zecchillo, C., Racagni, G., Riva, M.A., Molteni, R., 2013. Altered inflammatory responsiveness in serotonin transporter mutant rats. J. Neuroinflammation 10, 116. https://doi.org/10.1186/1742-2094-10-116

Macgillivray, L., Reynolds, K.B., Sickand, M., Rosebush, P.I., Mazurek, M.F., 2011. Inhibition of the serotonin transporter induces microglial activation and downregulation of dopaminergic neurons in the substantia nigra. Synapse 65, 1166-1172. https://doi.org/10.1002/syn.20954

Maes, M., Mihaylova, I., Kubera, M., Uytterhoeven, M., Vrydags, N., Bosmans, E., 2011. Lower whole blood glutathione peroxidase (GPX) activity in depression, but not in myalgic encephalomyelitis / chronic fatigue syndrome: another pathway that may be associated with coronary artery disease and neuroprogression in depression. Neuro Endocrinol. Lett. 32, $133-40$.

Maes, M., Mihaylova, I., Kubera, M., Uytterhoeven, M., Vrydags, N., Bosmans, E., 2009. Increased 8-hydroxy-deoxyguanosine, a marker of oxidative damage to DNA, in major depression and myalgic encephalomyelitis / chronic fatigue syndrome. Neuro Endocrinol. Lett. 30, $715-22$.

Malki, K., Pain, O., Tosto, M.G., Du Rietz, E., Carboni, L., Schalkwyk, L.C., 2015. Identification of genes and gene pathways associated with major depressive disorder by integrative brain analysis of rat and human prefrontal cortex transcriptomes. Transl. Psychiatry 5, e519. https://doi.org/10.1038/tp.2015.15

Marchesini, G., Petta, S., Dalle Grave, R., 2016. Diet, weight loss, and 
liver health in nonalcoholic fatty liver disease: Pathophysiology, evidence, and practice. Hepatology 63, 2032-2043. https://doi.org/10.1002/hep.28392

Marshall, J.A., Bessesen, D.H., 2002. Dietary Fat and the Development of Type 2 Diabetes. Diabetes Care 25, 620-622. https://doi.org/10.2337/diacare.25.3.620

Martinez, J.M., Garakani, A., Yehuda, R., Gorman, J.M., 2012. Proinflammatory and "resiliency" proteins in the CSF of patients with major depression. Depress. Anxiety 29, 32-38. https://doi.org/10.1002/da.20876

Mayes, S.D., Calhoun, S.L., Crowell, E.W., 2000. Learning Disabilities and ADHD. J. Learn. Disabil. 33, 417-424. https://doi.org/10.1177/002221940003300502

McArdle, M.A., Finucane, O.M., Connaughton, R.M., McMorrow, A.M., Roche, H.M., 2013. Mechanisms of Obesity-Induced Inflammation and Insulin Resistance: Insights into the Emerging Role of Nutritional Strategies. Front. Endocrinol. (Lausanne). 4. https://doi.org/10.3389/fendo.2013.00052

Mendoza, C., Matheus, N., Iceta, R., Mesonero, J.E., Alcalde, A.I., 2009. Lipopolysaccharide induces alteration of serotonin transporter in human intestinal epithelial cells. Innate Immun. 15, 243-250. https://doi.org/10.1177/1753425909104781

Michel, T.M., Camara, S., Tatschner, T., Frangou, S., Sheldrick, A.J., Riederer, P., Grünblatt, E., 2010. Increased xanthine oxidase in the thalamus and putamen in depression. World J. Biol. Psychiatry 11, 314-320. https://doi.org/10.3109/15622970802123695

Michel, T.M., Frangou, S., Thiemeyer, D., Camara, S., Jecel, J., Nara, K., Brunklaus, A., Zoechling, R., Riederer, P., 2007. Evidence for oxidative stress in the frontal cortex in patients with recurrent depressive disorder - a postmortem study. Psychiatry Res. 151, 145-150. https://doi.org/10.1016/j.psychres.2006.04.013

Moon, D.-O., Kim, K.-C., Jin, C.-Y., Han, M.-H., Park, C., Lee, K.-J., Park, Y.-M., Choi, Y.H., Kim, G.-Y., 2007. Inhibitory effects of eicosapentaenoic acid on lipopolysaccharide-induced activation in BV2 microglia. Int. Immunopharmacol. $\quad 7, \quad 222-229$. https://doi.org/10.1016/j.intimp.2006.10.001

Mootha, V.K., Lindgren, C.M., Eriksson, K.-F., Subramanian, A., Sihag, S., Lehar, J., Puigserver, P., Carlsson, E., Ridderstråle, M., Laurila, E., Houstis, N., Daly, M.J., Patterson, N., Mesirov, J.P., Golub, T.R., Tamayo, P., Spiegelman, B., Lander, E.S., Hirschhorn, J.N., Altshuler, D., Groop, L.C., 2003. PGC-1 $\alpha$-responsive genes involved in oxidative phosphorylation 
are coordinately downregulated in human diabetes. Nat. Genet. 34, 267-273. https://doi.org/10.1038/ng1180

Morgan, J.T., Chana, G., Pardo, C.A., Achim, C., Semendeferi, K., Buckwalter, J., Courchesne, E., Everall, I.P., 2010. Microglial Activation and Increased Microglial Density Observed in the Dorsolateral Prefrontal Cortex in Autism. Biol. Psychiatry 68, 368-376. https://doi.org/10.1016/j.biopsych.2010.05.024

Murphy, D.L., Fox, M.A., Timpano, K.R., Moya, P.R., Ren-Patterson, R., Andrews, A.M., Holmes, A., Lesch, K.-P., Wendland, J.R., 2008. How the serotonin story is being rewritten by new gene-based discoveries principally related to SLC6A4, the serotonin transporter gene, which functions to influence all cellular serotonin systems. Neuropharmacology 55, 932-60. https://doi.org/10.1016/j.neuropharm.2008.08.034

Murphy, E.J., 2015. Blood-brain barrier and brain fatty acid uptake: Role of arachidonic acid and PGE 2. J. Neurochem. 135, 845-848. https://doi.org/10.1111/jnc.13289

Nadeem, A., Ahmad, S.F., Bakheet, S.A., Al-Harbi, N.O., AL-Ayadhi, L.Y., Attia, S.M., Zoheir, K.M.A., 2017. Toll-like receptor 4 signaling is associated with upregulated NADPH oxidase expression in peripheral T cells of children with autism. Brain. Behav. Immun. 61, 146-154. https://doi.org/10.1016/j.bbi.2016.12.024

Necela, B.M., Su, W., Thompson, E.A., 2008. Toll-like receptor 4 mediates cross-talk between peroxisome proliferator-activated receptor $\gamma$ and nuclear factor- $\chi \mathrm{B}$ in macrophages. Immunology 125, 344-358. https://doi.org/10.1111/j.1365-2567.2008.02849.x

Nguyen, L.N., Ma, D., Shui, G., Wong, P., Cazenave-Gassiot, A., Zhang, X., Wenk, M.R., Goh, E.L.K., Silver, D.L., 2014. Mfsd2a is a transporter for the essential omega-3 fatty acid docosahexaenoic acid. Nature 509, 503-506. https://doi.org/10.1038/nature13241

Nguyen, M.T.A., Favelyukis, S., Nguyen, A.-K., Reichart, D., Scott, P.A., Jenn, A., Liu-Bryan, R., Glass, C.K., Neels, J.G., Olefsky, J.M., 2007. A Subpopulation of Macrophages Infiltrates Hypertrophic Adipose Tissue and Is Activated by Free Fatty Acids via Toll-like Receptors 2 and 4 and JNKdependent Pathways. J. Biol. Chem. 282, 35279-35292. https://doi.org/10.1074/jbc.M706762200

Nguyen, T.L.A., Vieira-Silva, S., Liston, A., Raes, J., 2015. How informative is the mouse for human gut microbiota research? Dis. Model. Mech. 8, 1-16. https://doi.org/10.1242/dmm.017400 
Nierenberg, A.A., Ghaznavi, S.A., Sande Mathias, I., Ellard, K.K., Janos, J.A., Sylvia, L.G., 2018. Peroxisome Proliferator-Activated Receptor Gamma Coactivator-1 Alpha as a Novel Target for Bipolar Disorder and Other Neuropsychiatric Disorders. Biol. Psychiatry 83, 761-769. https://doi.org/10.1016/j.biopsych.2017.12.014

Nisbett, K.E., Pinna, G., 2018. Emerging Therapeutic Role of PPAR- $\alpha$ in Cognition and Emotions. Front. Pharmacol. 9, 998. https://doi.org/10.3389/fphar.2018.00998

Nonogaki, K., Nozue, K., Oka, Y., 2006. Hyperphagia Alters Expression of Hypothalamic 5-HT2C and 5-HT1B Receptor Genes and Plasma Des-Acyl Ghrelin Levels in A y Mice. Endocrinology 147, 5893-5900. https://doi.org/10.1210/en.2006-0418

O'Brien, G., Pearson, J., 2004. Autism and learning disability. Autism 8, 125-40. https://doi.org/10.1177/1362361304042718

O’Dushlaine, C., Rossin, L., Lee, P., Duncan, L., Parikshak, N., Al, E., 2015. Psychiatric genome-wide association study analyses implicate neuronal, immune and histone pathways. Nat. Neurosci. 18, 199-209. https://doi.org/10.1038/nn.3922

Oades, R.D., Dauvermann, M.R., Schimmelmann, B.G., Schwarz, M.J., Myint, A.-M., 2010. Attention-deficit hyperactivity disorder (ADHD) and glial integrity: S100B, cytokines and kynurenine metabolism - effects of medication. Behav. Brain Funct. 6, 29. https://doi.org/10.1186/1744-9081-629

Oliveira, G., Diogo, L., Grazina, M., Garcia, P., Ataíde, A., Marques, C., Miguel, T., Borges, L., Vicente, A., Oliveira, C., 2005. Mitochondrial dysfunction in autism spectrum disorders: a population-based study. Dev. Med. Child Neurol. 47, 185-189. https://doi.org/10.1017/S0012162205000332

Orr, J.S., Puglisi, M.J., Ellacott, K.L.J., Lumeng, C.N., Wasserman, D.H., Hasty, A.H., 2012. Toll-like Receptor 4 Deficiency Promotes the Alternative Activation of Adipose Tissue Macrophages. Diabetes 61, 2718-2727. https://doi.org/10.2337/db11-1595

Osborn, O., Olefsky, J.M., 2012. The cellular and signaling networks linking the immune system and metabolism in disease. Nat. Med. 18, 363374. https://doi.org/10.1038/nm.2627

Oztop, D., Altun, H., Baskol, G., Ozsoy, S., 2012. Oxidative stress in children with attention deficit hyperactivity disorder. Clin. Biochem. 45, 7458. https://doi.org/10.1016/j.clinbiochem.2012.03.027

Ozturk, O., Basay, O., Basay, B.K., Alacam, H., Buber, A., Kaptanoglu, 
B., Enli, Y., Dogan, M., Tuncer, O.F., Kardesler, A.C., 2016. Oxidative Imbalance in Children and Adolescents with Autism Spectrum Disorder. Klin. Psikofarmakol. Bülteni-Bulletin Clin. Psychopharmacol. 26, 257-264. https://doi.org/10.5455/bcp.20160323105909

Pandey, G.N., Rizavi, H.S., Ren, X., Bhaumik, R., Dwivedi, Y., 2014. Toll-like receptors in the depressed and suicide brain. J. Psychiatr. Res. 53, 62-8. https://doi.org/10.1016/j.jpsychires.2014.01.021

Papadopoulos, M.., Koumenis, I.., Yuan, T.., Giffard, R.., 1997. Increasing vulnerability of astrocytes to oxidative injury with age despite constant antioxidant defenses. Neuroscience 82, 915-925. https://doi.org/10.1016/S0306-4522(97)00320-5

Park, B.S., Lee, J.-O., 2013. Recognition of lipopolysaccharide pattern by TLR4 complexes. Exp. Mol. Med. 45, e66-e66. https://doi.org/10.1038/emm.2013.97

Paşca, S.P., Nemeş, B., Vlase, L., Gagyi, C.E., Dronca, E., Miu, A.C., Dronca, M., 2006. High levels of homocysteine and low serum paraoxonase 1 arylesterase activity in children with autism. Life Sci. 78, 2244-2248. https://doi.org/10.1016/j.lfs.2005.09.040

Patching, S.G., 2017. Glucose Transporters at the Blood-Brain Barrier: Function, Regulation and Gateways for Drug Delivery. Mol. Neurobiol. 54, 1046-1077. https://doi.org/10.1007/s12035-015-9672-6

Patel, P.S., Buras, E.D., Balasubramanyam, A., 2013. The Role of the Immune System in Obesity and Insulin Resistance. J. Obes. 2013, 1-9. https://doi.org/10.1155/2013/616193

Peet, M., Murphy, B., Shay, J., Horrobin, D., 1998. Depletion of Omega3 Fatty Acid Levels in Red Blood Cell Membranes of Depressive Patients. Biol. Psychiatry 43, 315-319. https://doi.org/10.1016/S0006-3223(97)002060

Pelsser, L.M., Frankena, K., Toorman, J., Rodrigues Pereira, R., 2017. Diet and ADHD, Reviewing the Evidence: A Systematic Review of MetaAnalyses of Double-Blind Placebo-Controlled Trials Evaluating the Efficacy of Diet Interventions on the Behavior of Children with ADHD. PLoS One 12, e0169277. https://doi.org/10.1371/journal.pone.0169277

Peretti, S., Mariano, M., Mazzocchetti, C., Mazza, M., Pino, M.C., Verrotti Di Pianella, A., Valenti, M., 2018. Diet: the keystone of autism spectrum disorder? Nutr. Neurosci. 1-15. https://doi.org/10.1080/1028415X.2018.1464819

Perlman, R.L., 2016. Mouse Models of Human Disease: An Evolutionary 
Perspective. Evol. Med. Public Heal. eow014. https://doi.org/10.1093/emph/eow014

Pomytkin, I., Costa-Nunes, J.P., Kasatkin, V., Veniaminova, E., Demchenko, A., Lyundup, A., Lesch, K.-P., Ponomarev, E.D., Strekalova, T., 2018. Insulin receptor in the brain: Mechanisms of activation and the role in the CNS pathology and treatment. CNS Neurosci. Ther. https://doi.org/10.1111/cns.12866

Pomytkin, I.A., Cline, B.H., Anthony, D.C., Steinbusch, H.W., Lesch, K.P., Strekalova, T., 2015. Endotoxaemia resulting from decreased serotonin tranporter (5-HTT) function: a reciprocal risk factor for depression and insulin resistance? Behav. Brain Res. 276, 111-7. https://doi.org/10.1016/j.bbr.2014.04.049

Popa-Wagner, A., Mitran, S., Sivanesan, S., Chang, E., Buga, A.-M., 2013. ROS and brain diseases: the good, the bad, and the ugly. Oxid. Med. Cell. Longev. 2013, 963520. https://doi.org/10.1155/2013/963520

Rommelse, N., Franke, B., Geurts, H.M., Hartman, C.A., Buitelaar, J.K., 2010. Shared heritability of attention-deficit/hyperactivity disorder and autism spectrum disorder. Eur. Child Adolesc. Psychiatry 19, 281-95. https://doi.org/10.1007/s00787-010-0092-x

Rommelse, N.N.J., Franke, B., Geurts, H.M., Hartman, C.A., Buitelaar, J.K., 2010. Shared heritability of attention-deficit/hyperactivity disorder and autism spectrum disorder. Eur. Child Adolesc. Psychiatry 19, 281-295. https://doi.org/10.1007/s00787-010-0092-x

Rommelse, N.N.J., Geurts, H.M., Franke, B., Buitelaar, J.K., Hartman, C.A., 2011. A review on cognitive and brain endophenotypes that may be common in autism spectrum disorder and attention-deficit/hyperactivity disorder and facilitate the search for pleiotropic genes. Neurosci. Biobehav. Rev. 35, 1363-1396. https://doi.org/10.1016/j.neubiorev.2011.02.015

Rose, S., Melnyk, S., Pavliv, O., Bai, S., Nick, T.G., Frye, R.E., James, S.J., 2012. Evidence of oxidative damage and inflammation associated with low glutathione redox status in the autism brain. Transl. Psychiatry 2, e134. https://doi.org/10.1038/tp.2012.61

Rosenthal, N., Brown, S., 2007. The mouse ascending: perspectives for human-disease models. Nat. Cell Biol. 9, 993-999. https://doi.org/10.1038/ncb437

Ross, B.M., McKenzie, I., Glen, I., Bennett, C.P.W., 2003. Increased Levels of Ethane, A Non-invasive Marker of n-3 Fatty Acid Oxidation, in Breath of Children with Attention Deficit Hyperactivity Disorder. Nutr. 
Neurosci. 6, 277-281. https://doi.org/10.1080/10284150310001612203

Ruchi, K., Saxena, A.K., Sunil, G., Bashir, A., Prabhat, S., 2011. Antioxidant activity in children with ADHD-a comparison in untreated and treated subjects with normal children. Int. Med. J. Malaysia 10, 31-35.

Russo, A.J., 2010. Decreased Serum Cu/Zn SOD Associated with High Copper in Children with Attention Deficit Hyperactivity Disorder (ADHD). J. Cent. Nerv. Syst. Dis. 2, 9-14. https://doi.org/10.4137/jcnsd.s4553

Sanctuary, M.R., Kain, J.N., Angkustsiri, K., German, J.B., 2018. Dietary Considerations in Autism Spectrum Disorders: The Potential Role of Protein Digestion and Microbial Putrefaction in the Gut-Brain Axis. Front. Nutr. 5. https://doi.org/10.3389/fnut.2018.00040

Sarandol, A., Sarandol, E., Eker, S.S., Erdinc, S., Vatansever, E., Kirli, S., 2007. Major depressive disorder is accompanied with oxidative stress: short-term antidepressant treatment does not alter oxidative-antioxidative systems. Hum. Psychopharmacol. Clin. Exp. 22, 67-73. https://doi.org/10.1002/hup.829

Schmitt, A., Malchow, B., Hasan, A., Falkai, P., 2014. The impact of environmental factors in severe psychiatric disorders. Front. Neurosci. 8. https://doi.org/10.3389/fnins.2014.00019

Schnieder, T.P., Trencevska, I., Rosoklija, G., Stankov, A., Mann, J.J., Smiley, J., Dwork, A.J., 2014. Microglia of Prefrontal White Matter in Suicide. J. Neuropathol. Exp. Neurol. 73, 880-890. https://doi.org/10.1097/NEN.0000000000000107

Setiawan, E., Attwells, S., Wilson, A.A., Mizrahi, R., Rusjan, P.M., Miler, L., Xu, C., Sharma, S., Kish, S., Houle, S., Meyer, J.H., 2018. Association of translocator protein total distribution volume with duration of untreated major depressive disorder: a cross-sectional study. The Lancet Psychiatry 5, 339-347. https://doi.org/10.1016/S2215-0366(18)30048-8

Setiawan, E., Wilson, A.A., Mizrahi, R., Rusjan, P.M., Miler, L., Rajkowska, G., Suridjan, I., Kennedy, J.L., Rekkas, P.V., Houle, S., Meyer, J.H., 2015. Role of translocator protein density, a marker of neuroinflammation, in the brain during major depressive episodes. JAMA psychiatry 72, 268-75. https://doi.org/10.1001/jamapsychiatry.2014.2427

Sezen, H., Kandemir, H., Savik, E., Basmacı Kandemir, S., Kilicaslan, F., Bilinc, H., Aksoy, N., 2016. Increased oxidative stress in children with attention deficit hyperactivity disorder. Redox Rep. 21, 248-253. https://doi.org/10.1080/13510002.2015.1116729

Söğüt, S., Zoroğlu, S.S., Özyurt, H., Ramazan Yılmaz, H., Özuğurlu, F., 
Sivaslı, E., Yetkin, Ö., Yanık, M., Tutkun, H., Savaş, H.A., Tarakçığlu, M., Akyol, Ö., 2003. Changes in nitric oxide levels and antioxidant enzyme activities may have a role in the pathophysiological mechanisms involved in autism. Clin. Chim. Acta 331, 111-117. https://doi.org/10.1016/S00098981(03)00119-0

Sookoian, S., Gemma, C., García, S.I., Fernández Gianotti, T., Dieuzeide, G., Roussos, A., Tonietti, M., Trifone, L., Kanevsky, D., González, C.D., Pirola, C.J., 2007. Short Allele of Serotonin Transporter Gene Promoter Is a Risk Factor for Obesity in Adolescents*. Obesity 15, 271-276. https://doi.org/10.1038/oby.2007.519

Stanhope, K.L., 2016. Sugar consumption, metabolic disease and obesity: The state of the controversy. Crit. Rev. Clin. Lab. Sci. 53, 52-67. https://doi.org/10.3109/10408363.2015.1084990

Steiner, J., Bielau, H., Brisch, R., Danos, P., Ullrich, O., Mawrin, C., Bernstein, H.-G., Bogerts, B., 2008. Immunological aspects in the neurobiology of suicide: Elevated microglial density in schizophrenia and depression is associated with suicide. J. Psychiatr. Res. 42, 151-157. https://doi.org/10.1016/j.jpsychires.2006.10.013

Stinson, E.J., Piaggi, P., Ibrahim, M., Venti, C., Krakoff, J., Votruba, S.B., 2018. High Fat and Sugar Consumption During Ad Libitum Intake Predicts Weight Gain. Obesity 26, 689-695. https://doi.org/10.1002/oby.22124

Strekalova, T., Costa-Nunes, J.P., Veniaminova, E., Kubatiev, A., Lesch, K.-P., Chekhonin, V.P., Evans, M.C., Steinbusch, H.W.M., 2016. Insulin receptor sensitizer, dicholine succinate, prevents both Toll-like receptor 4 (TLR4) upregulation and affective changes induced by a high-cholesterol diet in mice. J. Affect. Disord. 196, 109-116. https://doi.org/10.1016/j.jad.2016.02.045

Strekalova, T., Evans, M., Costa-Nunes, J., Bachurin, S., Yeritsyan, N., Couch, Y., Steinbusch, H.M.W., Köhler, S.E., Lesch, K.-P., Anthony, D.C., 2015. Tlr4 upregulation in the brain accompanies depression- and anxietylike behaviors induced by a high-cholesterol diet. Brain. Behav. Immun. 48 . https://doi.org/10.1016/j.bbi.2015.02.015

Sullivan, E.L., Nousen, E.K., Chamlou, K.A., 2014. Maternal high fat diet consumption during the perinatal period programs offspring behavior. Physiol. Behav. 123, 236-42. https://doi.org/10.1016/j.physbeh.2012.07.014

Suzuki, K., Sugihara, G., Ouchi, Y., Nakamura, K., Futatsubashi, M., Takebayashi, K., Yoshihara, Y., Omata, K., Matsumoto, K., Tsuchiya, K.J., 
Iwata, Y., Tsujii, M., Sugiyama, T., Mori, N., 2013. Microglial Activation in Young Adults With Autism Spectrum Disorder. JAMA Psychiatry 70, 49. https://doi.org/10.1001/jamapsychiatry.2013.272

Sweeten, T.L., Posey, D.J., Shankar, S., McDougle, C.J., 2004. High nitric oxide production in autistic disorder: a possible role for interferon- $\gamma$. Biol. Psychiatry 55, 434-437. https://doi.org/10.1016/j.biopsych.2003.09.001

Talarowska, M., Gałecki, P., Maes, M., Gardner, A., Chamielec, M., Orzechowska, A., Bobińska, K., Kowalczyk, E., 2012. Malondialdehyde plasma concentration correlates with declarative and working memory in patients with recurrent depressive disorder. Mol. Biol. Rep. 39, 5359-5366. https://doi.org/10.1007/s11033-011-1335-8

Taurines, R., Schwenck, C., Westerwald, E., Sachse, M., Siniatchkin, M., Freitag, C., 2012. ADHD and autism: differential diagnosis or overlapping traits? A selective review. ADHD Atten. Deficit Hyperact. Disord. 4, 115139. https://doi.org/10.1007/s12402-012-0086-2

Tian, Y., Zhang, Y., Zhang, R., Qiao, S., Fan, J., 2015. Resolvin D2 recovers neural injury by suppressing inflammatory mediators expression in lipopolysaccharide-induced Parkinson's disease rat model. Biochem. Biophys. Res. Commun. 460, 799-805. https://doi.org/10.1016/j.bbrc.2015.03.109

Tong, L., Wang, L., Yao, S., Jin, L., Yang, J., Zhang, Y., Ning, G., Zhang, Z., 2019. PPAR $\delta$ attenuates hepatic steatosis through autophagy-mediated fatty acid oxidation. Cell Death Dis. 10, 197. https://doi.org/10.1038/s41419019-1458-8

Tontonoz, P., Spiegelman, B.M., 2008. Fat and Beyond: The Diverse Biology of PPAR r. Annu. Rev. Biochem. 77, 289-312. https://doi.org/10.1146/annurev.biochem.77.061307.091829

Torres-Platas, S.G., Cruceanu, C., Chen, G.G., Turecki, G., Mechawar, N., 2014. Evidence for increased microglial priming and macrophage recruitment in the dorsal anterior cingulate white matter of depressed suicides. Brain. Behav. Immun. 42, 50-59. https://doi.org/10.1016/j.bbi.2014.05.007

Tremblay, M.-E., Zhang, I., Bisht, K., Savage, J.C., Lecours, C., Parent, M., Titorenko, V., Maysinger, D., 2016. Remodeling of lipid bodies by docosahexaenoic acid in activated microglial cells. J. Neuroinflammation 13, 116. https://doi.org/10.1186/s12974-016-0580-0

Troutman, T.D., Bazan, J.F., Pasare, C., 2012. Toll-like receptors, signaling adapters and regulation of the pro-inflammatory response by PI3K. Cell Cycle 11, 3559-3567. https://doi.org/10.4161/cc.21572 
Umhau, J.C., Zhou, W., Carson, R.E., Rapoport, S.I., Polozova, A., Demar, J., Hussein, N., Bhattacharjee, A.K., Ma, K., Esposito, G., Majchrzak, S., Herscovitch, P., Eckelman, W.C., Kurdziel, K.A., Salem, N., 2009. Imaging incorporation of circulating docosahexaenoic acid into the human brain using positron emission tomography. J. Lipid Res. 50, 12591268. https://doi.org/10.1194/jlr.M800530-JLR200

Valdearcos, M., Robblee, M.M., Benjamin, D.I., Nomura, D.K., Xu, A.W., Koliwad, S.K., 2014. Microglia Dictate the Impact of Saturated Fat Consumption on Hypothalamic Inflammation and Neuronal Function. Cell Rep. 9, 2124-2138. https://doi.org/10.1016/j.celrep.2014.11.018

van den Hove, D.L.A., Van den Hove, D., Jakob, S.B., Schraut, K.-G., Kenis, G., Schmitt, A.G., Kneitz, S., Scholz, C.-J., Wiescholleck, V., Ortega, G., Prickaerts, J., Steinbusch, H., Lesch, K.-P., 2011. Differential effects of prenatal stress in 5-Htt deficient mice: towards molecular mechanisms of gene $\times$ environment interactions. PLoS One 6, e22715. https://doi.org/10.1371/journal.pone.0022715

Van Dyken, P., Lacoste, B., 2018. Impact of Metabolic Syndrome on Neuroinflammation and the Blood-Brain Barrier. Front. Neurosci. 12. https://doi.org/10.3389/fnins.2018.00930

Vargas, D.L., Nascimbene, C., Krishnan, C., Zimmerman, A.W., Pardo, C.A., 2005. Neuroglial activation and neuroinflammation in the brain of patients with autism. Ann. Neurol. 57, 67-81. https://doi.org/10.1002/ana.20315

Veniaminova E., Cespuglio R., Chernukha I., Schmitt-Boehrer A.G., Morozov S., Kalueff A.V., Kuznetsova O., Lesch K.P., Anthony D.C., Strekalova T. Metabolic, molecular and behavioural effects of Western diet in serotonin transporter-deficient mice: rescue by heterozygosity? Frontiers in Neuroscience. Under revision

Veniaminova, E., Cespuglio, R., Cheung, C.W., Umriukhin, A., Markova, N., Shevtsova, E., Lesch, K.-P., Anthony, D.C., Strekalova, T., 2017. AutismLike Behaviours and Memory Deficits Result from a Western Diet in Mice. Neural Plast. 2017, 1-14. https://doi.org/10.1155/2017/9498247

Veniaminova, E., Oplatchikova, M., Bettendorff, L., Kotenkova, E., Lysko, A., Vasilevskaya, E., Kalueff, A. V., Fedulova, L., Umriukhin, A., Lesch, K.-P., Anthony, D.C., Strekalova, T., 2020. Prefrontal cortex inflammation and liver pathologies accompany cognitive and motor deficits following Western diet consumption in non-obese female mice. Life Sci. 241, 117163. https://doi.org/10.1016/j.lfs.2019.117163 
Verma, P., Singh, A., Nthenge-Ngumbau, D.N., Rajamma, U., Sinha, S., Mukhopadhyay, K., Mohanakumar, K.P., 2016. Attention deficithyperactivity disorder suffers from mitochondrial dysfunction. BBA Clin. 6, 153-158. https://doi.org/10.1016/j.bbacli.2016.10.003

Visser, J.C., Rommelse, N.N.J., Greven, C.U., Buitelaar, J.K., 2016. Autism spectrum disorder and attention-deficit/hyperactivity disorder in early childhood: A review of unique and shared characteristics and developmental antecedents. Neurosci. Biobehav. Rev. 65, 229-263. https://doi.org/10.1016/j.neubiorev.2016.03.019

Wahli, W., Michalik, L., 2012. PPARs at the crossroads of lipid signaling and inflammation. Trends Endocrinol. Metab. 23, 351-363. https://doi.org/10.1016/j.tem.2012.05.001

Waku, T., Shiraki, T., Oyama, T., Maebara, K., Nakamori, R., Morikawa, K., 2010. The nuclear receptor PPAR $\gamma$ individually responds to serotoninand fatty acid-metabolites. EMBO J. 29, 3395-407. https://doi.org/10.1038/emboj.2010.197

Wang, X., Sundquist, K., Rastkhani, H., Palmér, K., Memon, A.A., Sundquist, J., 2017. Association of mitochondrial DNA in peripheral blood with depression, anxiety and stress- and adjustment disorders in primary health care patients. Eur. Neuropsychopharmacol. 27, 751-758. https://doi.org/10.1016/j.euroneuro.2017.06.001

Wu, L., Vikramadithyan, R., Yu, S., Pau, C., Hu, Y., Goldberg, I.J., Dansky, H.M., 2006. Addition of dietary fat to cholesterol in the diets of LDL receptor knockout mice: effects on plasma insulin, lipoproteins, and atherosclerosis. J. Lipid Res. 47, 2215-2222. https://doi.org/10.1194/jlr.M600146-JLR200

Yager, S., Forlenza, M.J., Miller, G.E., 2010. Depression and oxidative damage to lipids. Psychoneuroendocrinology 35, 1356-1362. https://doi.org/10.1016/j.psyneuen.2010.03.010

Yanik, M., Erel, O., Kati, M., 2004. The relationship between potency of oxidative stress and severity of depression. Acta Neuropsychiatr. 16, 200-203. https://doi.org/10.1111/j.0924-2708.2004.00090.x

Yin, J., Peng, Y., Wu, J., Wang, Y., Yao, L., 2014. Toll-like receptor 2/4 links to free fatty acid-induced inflammation and -cell dysfunction. J. Leukoc. Biol. 95, 47-52. https://doi.org/10.1189/jlb.0313143

Yiu, J.H.C., Dorweiler, B., Woo, C.W., 2017. Interaction between gut microbiota and toll-like receptor: from immunity to metabolism. J. Mol. Med. (Berl). 95, 13-20. https://doi.org/10.1007/s00109-016-1474-4 
Yoo, H.J., Park, M., Kim, S.A., 2017. Difference in mitochondrial DNA copy number in peripheral blood cells between probands with autism spectrum disorders and their unaffected siblings. World J. Biol. Psychiatry 18, 151-156. https://doi.org/10.1080/15622975.2016.1234069

Yorbik, O., Sayal, A., Akay, C., Akbiyik, D.I., Sohmen, T., 2002. Investigation of antioxidant enzymes in children with autistic disorder. Prostaglandins, Leukot. Essent. Fat. Acids 67, 341-343. https://doi.org/10.1054/plef.2002.0439

Young, A.M.H., Campbell, E., Lynch, S., Suckling, J., Powis, S.J., 2011. Aberrant NF-KappaB Expression in Autism Spectrum Condition: A Mechanism for Neuroinflammation. Front. Psychiatry 2. https://doi.org/10.3389/fpsyt.2011.00027

Young, J.J., Bruno, D., Pomara, N., 2014. A review of the relationship between proinflammatory cytokines and major depressive disorder. J. Affect. Disord. 169, 15-20. https://doi.org/10.1016/j.jad.2014.07.032

Yui, K., Tanuma, N., Yamada, H., Kawasaki, Y., 2017. Decreased total antioxidant capacity has a larger effect size than increased oxidant levels in urine in individuals with autism spectrum disorder. Environ. Sci. Pollut. Res. 24, 9635-9644. https://doi.org/10.1007/s11356-017-8595-3

Zand, H., Morshedzadeh, N., Naghashian, F., 2017. Signaling pathways linking inflammation to insulin resistance. Diabetes Metab. Syndr. Clin. Res. Rev. 11, S307-S309. https://doi.org/10.1016/j.dsx.2017.03.006

Zilkha, N., Kuperman, Y., Kimchi, T., 2017. High-fat diet exacerbates cognitive rigidity and social deficiency in the BTBR mouse model of autism. Neuroscience 345, 142-154.

https://doi.org/10.1016/j.neuroscience.2016.01.070

Zoroglu, S.S., Armutcu, F., Ozen, S., Gurel, A., Sivasli, E., Yetkin, O., Meram, I., 2004. Increased oxidative stress and altered activities of erythrocyte free radical scavenging enzymes in autism. Eur. Arch. Psychiatry Clin. Neurosci. 254. https://doi.org/10.1007/s00406-004-0456-7

Zoroğlu, S.S., Yürekli, M., Meram, İ., Söğüt, S., Tutkun, H., Yetkïn, Ö., Sïvasli, E., Savaş, H.A., Yanik, M., Herken, H., Akyol, Ö., 2003. Pathophysiological role of nitric oxide and adrenomedullin in autism. Cell Biochem. Funct. 21, 55-60. https://doi.org/10.1002/cbf.989 


\section{Summary}

In my work I investigated the behavioural, metabolic and molecular effects of a 'Western diet', a diet enriched with saturated fat, cholesterol and sugars, in wild type and mutant mice with genetic deficiency of the serotonin transporter (Sert). In the applied model, female mice were housed for 3 weeks on a diet containing $21 \%$ fat (including $12 \%$ saturated fat), $35 \%$ sugar, and $0.2 \%$ cholesterol. We found that young C57BL/6 mice fed with this diet displayed reduced social interactions, impairment in cognitive functions and motor coordination as well as depressive-like behaviour. These mice also showed decreased glucose tolerance and insulin resistance, dyslipidemia, and signs of non-alcoholic steatohepatitis-like syndrome. As SERT deficiency in humans is associated with increased risk of metabolic syndrome, especially during aging, we studied effects of the Western diet on mice heterozygous (HET) or complete knock-out (KO) for the gene encoding Sert.

We demonstrated that young mice housed on the Western diet display reduced scores of social interaction, increased dominant-like behaviours and signs of hyperactivity in a context of social interactions (Chapter 2). These changes were accompanied by decreased scores of fear conditioning and performance in the marble test, two paradigms for hippocampus-dependent behaviour, novel object recognition 
memory and increased depressive-like behaviour in the forced swim test. In addition, feeding with the Western diet impaired motor coordination in the rotarod and wire test, while no changes in muscle weight and strength or general locomotion were found. Behavioural changes in mice fed with the Western diet were accompanied by increased concentration of a marker of oxidative stress, malondialdehyde, signs of microglia activation, and decreased Sert gene expression in the prefrontal cortex (Chapters 2 and 3).

Based on the latter finding and well-known relationship between the variation in polymorphic region in the SERT gene (5-HTTLPR) in humans leading to its decreased activity and susceptibility to metabolic and mental alterations, we next studied how dietary challenge with Western diet interferes with Sert deficiency in mice (Chapter 4). We showed that complete genetic Sert inactivation in KO mice exacerbated consequences of the Western diet challenge in one-year-old female mice. These mice demonstrated greater decrease in glucose tolerance, increase in the expression of inflammation marker toll-like receptor 4 (Tlr4) and adverse behavioural changes compared to wild type mice housed on the Western diet. Remarkably, unlike wild type and $\mathrm{KO}$, mice HET for the Sert gene did not reveal hallmarks of the Western diet-induced syndrome, such as decrease in glucose tolerance, increased brain expression of Tlr4 and disrupted hippocampus-dependent performance. Yet, all Sert genotypes challenged with Western diet 
displayed similar changes in weight gain and increased scores of depressive-like behaviour in the swim test. Overall, our data indicated distinct effects of the Western diet on aging mice with complete versus partial Sert inactivation and suggested the importance of the interaction between Sert function and Western dietary pattern in the development of adverse metabolic and behavioural changes. Our data has led us to hypothesize that Western diet consumption can be an environmental factor that may interact with genetic risk factors of ASD and ADHD, increasing the risk for these disorders to manifest in adulthood. 


\section{Samenvatting}

\section{Het effect van het "westers dieet" op emotioneel, sociaal en cognitief gedrag, aangetoond in een studie met normale en serotonine transporter- deficiënte muizen}

In mijn werk onderzocht ik gedrags-, metabole en moleculaire effecten van het zogenaamde 'westerse dieet', een dieet verrijkt met verzadigd vet, cholesterol en suikers, bij muizen, inclusief mutanten met een genetische deficiëntie van serotonine transporter (Sert). In het toegepaste model werden vrouwelijke muizen gedurende 3 weken gehuisvest op een dieet dat $21 \%$ vet bevat, waaronder $12 \%$ verzadigd vet, $35 \%$ suiker en $0,2 \%$ cholesterol. We vonden bij jonge C57BL / 6 met dit dieet, verminderde sociale interacties, verminderde cognitieve functies en motorische coördinatie en verhoogd depressief gedrag, evenals een verminderde glucosetolerantie en insulineresistentie, dyslipidemie en tekenen van niet-alcoholische steatohepatitis-achtige syndroom. Aangezien Sert-deficiëntie bij mensen wordt geassocieerd met een verhoogd risico op het metabool syndroom, vooral bij vrouwen tijdens veroudering, hebben we de effecten bestudeerd van het westerse dieet op heterozygote muizen (HET) of op muizen met een volledige knock-out (KO) voor het gen dat voor Sert codeert. 
We toonden aan dat jonge muizen met dit westers dieet lager scoorden op sociale interactie, dominant gedrag en tekenen van hyperactiviteit in de context van sociale interacties vertoonden (Hoofdstuk 2). Deze veranderingen gingen gepaard met verminderde scores van angstconditionering en verminderde prestaties gemeten met de knikkertest, de twee paradigma's voor hippocampus afhankelijk gedrag, het herkennen van nieuwe voorwerpen en een verhoogd depressief gedrag in de geforceerde zwemtest. Bovendien verminderde het westerse dieet de motorische coördinatie gemeten met de rotaroden de draadtest, terwijl er geen veranderingen werden waargenomen in het spiergewicht en de spierkracht of in de algemene motoriek. Gedragsveranderingen bij muizen die met een westers dieet werden gevoed, gingen gepaard met een verhoogde concentratie van malondialdehyde, een marker van oxidatieve stress, en tekenen van microglia-activering en verminderde Sert-genexpressie in de prefrontale cortex (Hoofdstukken 2 en 3).

Op basis van deze bevindingen en de bekende relatie tussen de variatie in polymorfe regio in het Sert-gen (5-HTTLPR) bij mensen wat aanleiding geeft tot verminderde activiteit en gevoeligheid voor metabole en affectieve tekorten, hebben we vervolgens naar de interferentie van het westers dieet op Sert-deficientie bij de onderzochte muizen (Hoofdstuk 4). We toonden aan dat de volledige genetische inactivering van Sert bij KO-muizen de gevolgen van de 
westerse voedingsproblematiek bij vrouwelijke muizen van één jaar oud verergerde. Deze muizen vertoonden een grotere afname in glucosetolerantie, een toename in de hypothalamische expressie van de ontstekingsmarker tol-like receptor 4 (Tlr4) en gedragsveranderingen in verghelijking met wildtype muizen op een westers dieet.

In tegenstelling tot de wildtype en $\mathrm{KO}$-muizen, vertoonden HET muizen niet de kenmerken van het door het westerse dieetgeïnduceerde syndroom, zoals afname van de glucosetolerantie, een verhoogde hersenexpressie van Tlr4 en verstoorde hippocampusafhankelijke prestaties. Toch vertoonden alle Sert-genotypen met een westers dieet vergelijkbare veranderingen in gewichtstoename en verhoogde scores van depressief gedrag in de zwemtest. Over het algemeen wezen onze gegevens op duidelijke effecten van het westers dieet op de verouderende muizen met een volledige versus gedeeltelijke Sert inactivering en suggereert het belang van de interactie tussen de Sert-functie en het westerse voedingspatroon bij de ontwikkeling van metabole en gedragveranderingen. Onze resultaten veronderstellen dat het westerse dieet een milieurisicofactor kan zijn welke kan interfereren met genetische factoren van een autisme-spectrum-stoornis (ASS) en een aandachts-tekort-stoornis met hyperactiviteit (ADHD) op volwassen leeftijd. 


\section{Valorization}

Relevance for society

Western-style foods consumption has been increasing steadily since the 1950s, especially in Western-culture countries. Due to a growing economy, higher income and faster pace of life, rates of Western diet consumption are also increasing nowadays in countries where such foods were never part of the traditional culture. Western diet is associated with metabolic disorders, such as obesity, type 2 diabetes and non-alcoholic fatty liver disease (NAFLD). Worldwide, the estimated prevalence for obesity and NAFLD is around 650 million and for type 2 diabetes more than 500 million people. The WHO anticipates that worldwide deaths from diabetes will double by 2030 .

Metabolic disorders are also often associated with increased risk of psychiatric disorders, including mood disorders, attentiondeficit/hyperactivity disorder (ADHD) and autism spectrum disorder (ASD). ASD and ADHD are complex neurodevelopmental disorders with the prevalence estimated $7 \%$ and $2 \%$ respectively, thus affecting more than 100 million people in the world. ASD may significantly limit the capacity of an individual to participate in society. Those affected may not be able to conduct day-to-day activities and require life-long care and support. Both ASD and ADHD negatively influence the person's educational and social attainments. These disorders cooccur 
with a high frequency with each other as well as with affective disorders such as depression.

Increasing prevalence of ASD and ADHD suggests an important role of environmental factors, including nutrition, in the development of these disorders. While adverse reactions to foods have often been reported in children with $\mathrm{ASD}$ or $\mathrm{ADHD}$, the impact of diet on ASD/ADHD associated behaviours in adulthood is much less well understood. Our study showed that in adult female mice, feeding with the Western diet induced behaviours phenomenologically similar to the behaviours associated with human ADHD and ASD. Thus, this work provides a rationale for future clinical studies on the effects of nutrition on ADHD and ASD syndromes in adulthood. Based on our results, it can be suggested that dietary interventions may lead to symptom relief in patients with ASD or ADHD, which will decrease costs associated with disability and health management.

We also found metabolic changes and signs of neuroinflammation and oxidative stress in the brains of mice fed with the Western diet. These data suggest that compounds with anti-inflammatory and antioxidative properties might be useful to ameliorate symptoms in cases ASD or ADHD that cooccur with metabolic disorders such as type 2 diabetes or NAFLD. The main molecular targets reported in this study are toll-like receptor 4 (Tlr4) and peroxisome proliferator-activated receptor gamma (PPAR $\gamma)$. Agonists and 
antagonists of these receptors are now being studied in relation to diabetes, obesity, inflammatory disease treatment, and our work may further increase the interest of the industry in these compounds by proposing new applications.

Decreased activity of serotonin transporter (SERT), which occurs in a large percentage of people due to the 5-HTTLPR polymorphism in the SERT gene, was extensively discussed as a factor of genetic predisposition to affective and neurodevelopmental disorders. Current work demonstrated that mice with partial Sert deficiency displayed a resilience to dietary-induced abnormalities in glucose tolerance, Tlr4 brain expression and hippocampus-dependent behaviour, aberrations that were present in mice with complete Sert deficiency and wild type animals after feeding with the Western diet. Based on our findings, it seems important to further study the consequences of interaction between adherence to a certain dietary pattern and the 5-HTTLPR polymorphism in humans, which might increase the need for genotyping services. Based on the genotype, further dietary counseling services as well as pharmacotherapy, such as use of compounds targeting TLR4, PPAR $\curlyvee$ or SERT might be advised.

\section{Target groups}

We consider our target groups could be 1) individuals with high adherence to the Western dietary pattern or patients with metabolic 
disorders, 2) adult patients with ASD, ADHD and depression as well individuals with high genetic risk for these disorders.

Activity / Products

Our findings highlight the importance of future clinical investigation of the possible effects of the Western diet in those with a high susceptibility to, or having been diagnosed with, ASD, ADHD and depression. Dietary recommendations in combination with 5-HTTLPR polymorphism genotyping might be suggested for these groups. In some cases, pharmacotherapy using inhibitors/antagonists of TLR4 and agonists of PPAR $\curlyvee$ might be used.

\section{Innovation}

The work presented herein has been innovative in various regards. First, we have shown that the Western diet mice can evoke behaviours reminiscent of ASD and ADHD symptoms, as well as impairment of emotionality in a mouse model. In particular, our studies were among the first to show that in adult mice, Western diet alters social behaviour, which is known to be affected both in ASD and ADHD. This result suggests dietary interventions as a promising treatment approach in adult patients with ASD and ADHD. Then, our model of the three-week Western diet feeding provides an opportunity to study behavioural and brain changes associated with metabolic syndrome as well as possible pharmacotherapy at low labor and time 
costs. Much longer periods of diet feeding have been employed routinely elsewhere in similar studies, leading to serious obesity. Further, we extended the knowledge of the Western det consequences on behavioural and brain molecular parameters in female mice, while in the past, predominately male rodents were used for this purpose. We also suggested important contributions of neuroinflammation, oxidative stress and altered Sert functioning in the discussed behavioural changes, which may direct pharmacotherapy development. And, finally, we investigated effects of the Western diet on metabolic, behavioural and molecular parameters in female mice with complete and partial deficiency of Sert. This has not been shown before, but is relevant to the human context regarding the 5-HTTLPR polymorphism, which also results in decreased SERT activity. We found that complete genetic Sert inactivation exacerbated metabolic alterations, neuroinflammation and behavioural consequences of the Western diet feeding in aging mice. On the contrary, mice with partial genetic Sert deficit displayed a "rescued" phenotype in dietary-induced abnormalities in glucose tolerance, Tlr4 brain expression and hippocampus-dependent behaviour, that was shown for the first time.

\section{Implementation}

Results of our study are relevant for scientific and medical communities and for the general public. From an academic perspective, 
results were presented at national and international conferences ( 8 oral and 3 poster presentations at the conferences including " 12 th Goettingen Meeting of the German Neuroscience Society", "20th EURON PhD Days", and "19th WPA World Congress of Psychiatry") and were or will be published in peer-reviewed international journals. Being a part of Eat2beNice project (Horizon 2020 EU Research and Innovation programme, grant No. 728018), the study was presented in the project reports and in the New Brain Nutrition Blog (newbrainnutrition.com/blog, blogs: "Why do we use mouse models in diet research?", "Nutrition and Psychiatry: experience of attending the 19th WPA World Congress of Psychiatry"). Our model of the Western diet feeding in mice is currently being used by colleagues from the food industry and for investigating a 'healthy' meat product. 


\section{Acknowledgments}

First of all, I would like to thank Prof. Klaus-Peter Lesch for the general supervision of my $\mathrm{PhD}$ project that was a joint research project between Maastricht University, Department of Neuroscience and I.M. Sechenov First Moscow State Medical University. Prof. Lesch provided me a unique opportunity to join the project supported by 'Eat2beNICE' grant as a part of Horizon 2020 EU Research and Innovation programme and '5-100 Russian Excellence Project'. During my $\mathrm{PhD}$ years I had a rare chance to participate in numerous studies, which resulted in several important publications, and this all would not be possible without irreplaceable contribution of Prof. Lesch. I am very thankful for his warm welcome in his laboratory and in his house in Wuerzburg where I had training at the very beginning of my $\mathrm{PhD}$. I'm grateful for Prof. Lesch for his willingness to establish high standards of research practice in the laboratory, for his criticism, attention, encouragement and support across the years 2014-2019.

I would like to express my gratitude to the head of School for Mental Health and Neuroscience Prof. David Linden, the head of Division III Prof. Jos Prickaerts, and administration of Maastricht University for the opportunity to defend my thesis and for general support. 
I express my special gratitude to Prof. Tatyana Strekalova, my immediate supervisor, who coached me across the years of my PhD. Prof. Strekalova provided me with unique research and training opportunities through her collaborations in the Universities of Oxford, Wuerzburg, Lisbon, Liege and Hong Kong. With her invaluable organizational help and support, I was lucky to attend international conferences and undergo trainings in the most prominent laboratories in the field of Neuroscience. During activities and traineeships arranged by Prof. Strekalova, I was able to learn important methods and carried out experiments for my Thesis. Prof. Strekalova gave me priceless experience of planning, study design, and organization of my experiments, communication with colleagues and presenting my data. She personally taught me many behavioural testing paradigms in mice, paying much attention to quality control and consistency of testing environment. Her attention to details and criticism helped me to improve my writing and reporting skills and to contribute to 7 peer-review papers over the period of $\mathrm{PhD}$ studies. I am very thankful for her constant support and for encouraging me to be productive and effective in my work. I trust it is impossible to overestimate the role of Prof. Tatyana Strekalova in the defense of my PhD Thesis.

I express my sincere gratitude to the co-supervisor of my thesis, Prof. Daniel C. Anthony. Thanks to him, I got an opportunity to study the methods of histology and immunohistochemistry in the University 
of Oxford and acquired the best research standards. Prof. Anthony's advice, discussions we had, his mentoring and help with manuscript writing are impossible to overestimate. I would like to especially thank Prof. Anthony, members of his laboratory and his family for their warm welcome in Oxford, where I am always glad to come back.

I am thankful to Prof. Lucien Bettendorff who welcomed me in Liege University and gave me important advices regarding data presentation, Dr. Angelika G. Schmitt-Boehrer for her help during my training in Wuerzburg University, Dr. Joao Costa-Nunes for his hospitality and support during my internships in Lisbon, Jay Roodselaar form Oxford University who kindly helped me to learn immunohistochemistry, Dr. Elena Shevtsova from Institute of Physiologically Active Compounds of Russian Academy of Sciences for her help with biochemical blood analysis, Jenna Hebert and Daniel Radford-Smith for the help with the experiments on serotonin transporter-deficient mice in Oxford University, Dr. Johannes de Munter for moral support and important advice on data presentation, Prof. Igor Pomytkin from Sechenov University for valuable advice on interpretation of my results, and Prof. Raymond Cespuglio from Claude Bernard University Lyon for his great interest in the study on serotonin transporter-deficient mice and help with organization of the experiments. I thank Prof. Eugene Ponomarev, Prof. Chi Wai Cheung and Prof. Lee Wei Lim and their students for the opportunity to share 
my results and get valuable comments as well as participate in the seminars and experiments in their laboratories during my stay in Hong Kong. I would like to thank my colleagues from the laboratory of Dr. Liliya Fedulova and Prof. Irina Chernukha from V.M. Gorbatov Federal Research Center for Food Systems of Russian Academy of Sciences (Moscow) for knowledge sharing during the ongoing collaborative project.

Also, I am pleased to thank Prof. Andrey Zamyatnin, Prof. Andrey Svystunov, Prof. Denis Butnaru, Elina Vinogradova and Prof. Alexei Umryukhin from the I.M. Sechenov First Moscow State Medical University for providing me research facilities and for their support.

I am very grateful for the help of my former and present colleagues: Dr. Nataliia Markova, Nataliia Bazhenova, Dr. Alexander Trofimov, Evgeniy Svirin, Margarita Oplatchikova, Diana Babaevskaya, and, especially, Anna Gorlova and Dr. Dmitrii Pavlov. During these years we were participating in numerous experiments, trainings and conferences, sharing the experiences I will always remember. I am pleased that $\mathrm{PhD}$ defenses of Anna, Dmitrii, and myself are planned for the same date so will share this moment as well.

I thank my dearest friends for their sensitive attention and for being tactful not to ask me each time we met if I am going to finish my $\mathrm{PhD}$ thesis soon. 
I would like to address my warmest thanks to my relatives: my parents, my grandparents, my brother and his family for encouraging my education throughout my life and my relatives-in-law for their support. I especially thank my beloved husband Ilya for helping me to find a right decision when I was hesitating and for his infinite patience.

I am thankful to all the people whom I encountered during these years, who even not knowing about it has led me to the moment I am in now. 


\section{About the author}

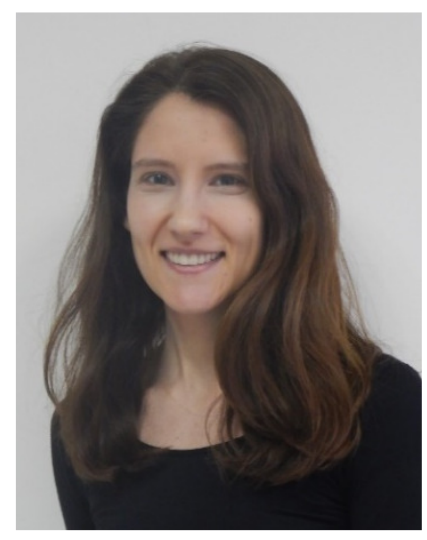

Ekaterina Veniaminova was born on November 17th, 1990 in Leningrad, USSR (now St. Petersburg, Russia). In 2008-2014, Ekaterina was studying at St. Petersburg State Polytechnical University, graduating first as a Bachelor of Science in physics in 2012 and then as a Master of Science in biophysics in 2014 . During her undergraduate studies, Ekaterina did an internship (under the supervision of Prof. Olga Zubareva) and was further employed as a laboratory assistant at the Department of Physiology, Institute of Experimental Medicine, a place where Ivan Pavlov was doing his groundbreaking work on the digestive systems of dogs. Starting with in vitro experiments, Ekaterina then became also interested in inflammation-induced behavioural changes in rodents and did her master research project in a rat model of the CNS neonatal pathology. In 2014, after graduating from St. Petersburg State Polytechnical University, Ekaterina moved to Moscow and started to work as a junior researcher under the supervision of Prof. Tatyana Strekalova, first in the Institute of General Pathology and Pathophysiology and then, when in 2016 a new laboratory directed by Prof. Klaus-Peter Lesch 
was established, in the I.M. Sechenov First Moscow State Medical University (Sechenov University).

Ekaterina became appointed as an external PhD student in Maastricht University in April 2015. She was doing her PhD research in a mouse model of the Western diet feeding under the immediate supervision of Prof. Tatyana Strekalova. Her work was also supervised by Prof. Klaus-Peter Lesch (University of Wuerzburg and University of Maastricht) and Prof. Daniel C. Anthony (University of Oxford). Ekaterina's research has been focusing on the metabolic, behavioural and molecular consequences of the Western diet feeding and the role of serotonin transporter deficiency in the diet-induced changes.

Ekaterina has acquired numerous techniques in behaviour testing, including tests for anxiety and depressive-like behaviour, social, motor and cognitive functions and stress models, brain dissection and surgery, metabolism assessment, histology and immunohistochemistry, as well as molecular biology through a number of local and international internships and collaborative research projects. During the internships in the New University of Lisbon (Portugal), Ekaterina underwent intensive training in behavioural methods under the supervision of Prof. Strekalova and got her first experience in working with transgenic mice. There, she was also trained in glucose tolerance assessment by Dr. Brandon Cline (Strasbourg University) and brain dissection by Prof. Anthony. She was studying immunohistochemistry, brain cutting 
and microscopy first in the laboratory of Prof. Lesch in University of Wuerzburg (Germany) and then in the laboratory of Prof. Anthony in University of Oxford (UK), where she investigated microglial activation in the brains of mice fed with the Western diet. In Liege University (Belgium) she studied fluorometric assays of oxidative stress measurement under the supervision of Prof. Lucien Bettendorff. Ekaterina spent almost a year in Hong Kong, participating in the laboratory activities in University of Hong Kong (Prof. Chi Wai Cheung) and Chinese University of Hong Kong (Prof. Eugene Ponomarev), where she was involved in behavioural phenotyping of new transgenic mouse lines. Recently, she took a part in a collaborative project with V.M. Gorbatov Federal Research Center for Food Systems of Russian Academy of Sciences (Moscow, Russia, Dr. Liliya Fedulova and Prof. Irina Chernukha) dedicated to the testing of a new 'healthy' meat product in a mouse model of the Western diet feeding.

Ekaterina is a coauthor of 11 research papers in peer-review jounals, including "CNS Neuroscience \& Therapeutics", "Brain, Behavior, and Immunity" and "Neural Plasticity". Her H-index is currently 4. She actively participates in local and international conferences and, among other conferences, she has presented her work at "12th Goettingen Meeting of the German Neuroscience Society" in 2017, "20th EURON PhD Days" and "Forum of European Neuroscience Societies" in 2018, and "19th WPA World Congress of Psychiatry" in 
2019. During her $\mathrm{PhD}$ studies she gave talks on her project in Universities of Maastricht, Wuerzburg, Liege, Oxford, and Hong Kong and in Sechenov University in Moscow. Ekaterina is a lab manager of the Laboratory of Psychiatric Neurobiology in Sechenov University, she supervises undergraduate students and writes blogs for the Eat2beNice project (newbrainnutrition.com/blog).

Tel: +7 (916) 8114361

E-mail: Katya.veniaminova@gmail.com

\section{Employment}

Since 11/2016 - Junior researcher, Laboratory of Psychiatric Neurobiology, Institute of Molecular Medicine, I.M. Sechenov First Moscow State Medical University, Moscow, Russia (1 fte).

Since 4/2015 - External PhD student, School for Mental Health and Neuroscience, European Graduate School of Neuroscience, Maastricht University, Maastricht, Netherlands (0 fte).

10/2014 - 10/2016 - Junior researcher, Laboratory of Cognitive Dysfunctions, Institute of General Pathology and Pathophysiology, Russian Academy of Medical Sciences, Moscow, Russia (1 fte). 9/2010 - 10/2014 - Laboratory assistant, Laboratory of Neurobiology of Integrative Functions of the Brain, Department of Physiology (Pavlov's), Institute of Experimental Medicine, Russian Academy of Medical Sciences, St. Petersburg, Russia (0.5 fte). 


\section{Training experiences in other research Institutes}

2/2019 (3 weeks). GIGA Neuroscience, Centre de Neurobiologie Cellulaire et Moléculaire, University of Liege, Liege, Belgium. Supervisor: Prof. L.Bettendorff. Training in Malondialdehyde assay. Project: Oxidative stress in the brain structures of mice fed the Western diet

9/2018 (2 weeks). Department of Pharmacology, Oxford University, Oxford, UK. Supervisor: Prof. D.Anthony. Behavioural testing and qPCR analysis. Project: Effects of the Western diet on behaviour and gene expression of mice with genetic deficiency of serotonin transporter 3/2017, 12/2017 (4 weeks). School of Biomedical Sciences, The Chinese University of Hong Kong, Hong Kong SAR. Supervisor: Prof. E.Ponomarev. Behavioural testing. Projects: Behavioural assessment of mice with genetic deficiency of ST3GAL5, Behavioural assessment of APP transgenic mice

1/2017, 10/2017, 3/2018 (10 weeks). Department of Pharmacology, Oxford University, Oxford, UK. Supervisor: Prof. D.Anthony. Training in histochemical and immunochistochemical staining. Project: The effects of Western diet on microglia activation in mouse brain 
9/2016 (2 weeks). School of Biomedical Sciences, The Chinese University of Hong Kong, Hong Kong SAR. Supervisor: Prof. E.Ponomarev. Training in flow cytometry. Project: Effects of the Western diet on platelet activation in mice

3/2015 (3 weeks). Laboratory of Translational Neuroscience, Department of Psychiatry, Psychosomatics and Psychotherapy, University of Wuerzburg, Wuerzburg, Germany. Supervisor: Prof. K.P.Lesch. Training in immunohistochemical staining, brain cutting, microscopy. Project: Study of potential protective effects of BDNF-1 fragment on stress-induced suppression of the hippocampal neurogenesis in a mouse

10/2014, 12/2014 (4 weeks). Institute of Hygiene and Tropical Medicine, New University of Lisbon, Lisbon, Portugal. Supervisors: Prof. T.Strekalova, Dr. B.Cline, Prof. D.Anthony. Training in behavioural methods, glucose tolerance test, brain perfusion and dissection, blood collection. Projects: Study of glucose tolerance, memory anxiety-and depressive-like behaviour in aged SERT-het mice, TPH2 mutants and aggressive behaviour in stressed male and female mice: genetic correlates 


\section{Conferences attended, given presentations and seminars}

1. Veniaminova E, Oplatchikova M, Gorlova A, Hebert J, RadfordSmith D, Cespuglio R, Schmitt-Boehrer A, Lesch K-P, Anthony D, Strekalova T. The role of genetic serotonin transporter deficiency in consequences of exposure to the Western diet: a study in mice // 19th WPA World Congress of Psychiatry, Lisbon, Portugal, 2324.08.2019 (poster presentation)

2. Veniaminova E. Mouse model of 'Western diet', pro-oxidative changes in the brain and impact of genetic serotonin transporter deficiency // "Inflammation and oxidative stress in translational research: new models and treatments", Estoril, Portugal, 20.08.2019 (oral presentation)

3. Veniaminova E. Behavioural and molecular effects of the Western diet in mice with genetic serotonin transporter (Sert) deficiency // UK-Russia Young Medic Conference, I.M. Sechenov First Moscow State Medical University, Moscow, Russia, 01.03.2019 (oral presentation)

4. Veniaminova E, Gorlova A, Lesh K-P, Anthony D, Strekalova T. The role of serotonin transporter deficiency in the consequences of Western diet in mice // Poster session at the Department of Normal Physiology, I.M. Sechenov First Moscow State Medical University, Moscow, Russia, 24.01.2019 (poster presentation) 
5. Veniaminova E. Behavioural, molecular and metabolic consequences of Western diet: a study on mice // Talk at Gorbatov Federal Research Center for Food Systems of RAS, Moscow, Russia, 26.12.2018 (oral presentation)

6. Veniaminova E. Behavioural, molecular and metabolic consequences of Western diet in mice and the role of genetic deficiency of the serotonin transporter // Talk at I.M. Sechenov First Moscow State Medical University, Moscow, Russia, 20.11.2018 (oral presentation)

7. Veniaminova E, Oplatchikova M, Gorlova A, Pomytkin I, Lesch K-P, Anthony D, Strekalova T. Behavioural, molecular and metabolic consequences of Western diet in mice and the role of genetic deficiency of the serotonin transporter // 20th EURON PhD Days, Brussels, Belgium, 13-14.09.2018 (oral presentation)

8. Veniaminova E, Kopeikina E, Zheng Kai S T, Khairuddin Sh, Lim L W, Ponomarev E, Strekalova T. Behavioural characteristics of KO mice lacking ST3GAL5, key enzyme in sialated ganglioside synthesis // Forum of European Neuroscience Societies, Berlin, Germany, 7-11.07.2018 (poster presentation)

9. Veniaminova E, Kopeikina E, Kai Tan SZ, Khairuddin Sh, Lesch K-P, Lim LW, Ponomarev E, Strekalova T. Behavioural characteristics of mice with genetic deficiency of ST3GAL5, the key enzyme in brain-specific ganglioside synthesis // Sechenov International 
Biomedical Summit, Moscow, Russia, 21.05.2018 (poster presentation)

10. Veniaminova E, Oplatchikova M, Gorlova A, Pavlov D, Bazhenova N, Pomytkin I, Lesch KP, Anthony D, Strekalova T. Increased impulsivity and microglia activation in the prefrontal cortex in mice housed on Western diet are associated with multiple behavioural abnormalities // 25th Annual International "Stress and Behavior" Neuroscience and Biopsychiatry Conference, St. Petersburg, Russia, 16-20.05.2018 (oral presentation)

11. Veniaminova E, Shevtsova E, Markova N, Gorlova A, Pavlov D, Morozova A, Chekhonin V, Lesch K-P, Anthony D, Strekalova T. Behavioural, molecular and metabolic consequences of Western diet and ameliorating effect of dicholine succinate // 12th Goettingen Meeting of the German Neuroscience Society, Goettingen, Germany, 22-25.04.2017 (poster presentation)

12. Veniaminova E. Behavioural, molecular and metabolic consequences of The effects of Western diet on behaviour and molecular parameters in mice // International Evaluation Conference of the Laboratory of Psychiatric Neurobiology, I.M. Sechenov First Moscow State Medical University, Moscow, Russia, 06.04.2017 (oral presentation)

13. Veniaminova E. Dietary cholesterol as a pathogenetic factor of depressive-like syndrome: a study on mice // Talk at Laboratory 
and Clinical Research Institute for Pain, The University of Hong Kong, 13.05.2016 (oral presentation)

14. 22nd Annual International "Stress and Behavior" Neuroscience and Biopsychiatry Conference, St. Petersburg, Russia, 16-19.05.2015 (attended)

15. 11th Goettingen Meeting of the German Neuroscience Society, Goettingen, Germany, 18-21.03.2015 (attended)

16. Veniaminova E. Dietary cholesterol and genetically decreased SERT function in mice: potential interaction in the pathogenesis of depressive-like syndrome // Division of Molecular Psychiatry, Laboratory of Translational Neuroscience, University Clinic of Wuerzburg, Wuerzburg, Germany, Laboratory Seminar, 12.03.2015 (oral presentation)

17. Zubareva OE, Veniaminova EA, Kalemenev SV, Schwarz AP, Fomalont KJ. Altered cognitive dysfunction and changes in NMDA and AMPA receptor subunit gene expression in rat brain after LPS injection in early postnatal ontogenesis // All-Russian Conference with International Participation "Neurochemical mechanisms of adaptive and pathological brain states", St. Petersburg, Russia, 2426.06.2014 (poster presentation)

18. Fomalont KJ, Veniaminova EA, Kalemenev SV, Trofimov AN, Schwarz AP, Zubareva OE. Early-life LPS administrations induce cognitive decline and changes in NMDA receptor subunit gene 
expression in the rodent brain // 21st Annual PNIRS Scientific Meeting, Philadelphia, USA, 28-31.05.2014

19. 21st Annual International "Stress and Behavior" Neuroscience and Biopsychiatry Conference, St. Petersburg, Russia, 16-19.05.2014 (attended)

20. Veniaminova EA, Rotov AYu. LPS effects on NMDA receptor subunit gene expression in brain structures of juvenile rats // International Scientific Conference of Students and Young Scientists "Lomonosov - 2014", Moscow, Russia, 7-11.04.2014 (poster presentation)

21. Veniaminova EA, Fomalont KJ, Manyukhina VO, Zubareva OE. The influence of bacterial lipopolysaccharide on NMDA receptor subunit gene expression in juvenile rat brain. All-Russian Conference of Young Scientists "Neurobiology of Integrative Brain Functions", St. Petersburg, Russia, 12-14.11.2013 (poster presentation)

22. Veniaminova EA, Trofimov AN, Schwarz AP, Zubareva OE. The influence of lipopolysaccharide injections in early postnatal ontogenesis on expression of NMDAreceptor subunits in rat brain structures // The XLI theoretical and practical conference with international participation «The week of science and academic research at SPBSPU», St. Petersburg, Russia, 3-8.12.2012 (oral presentation) 
23. Veniaminova EA, Trofimov AN, Schwarz AP. Changes in NMDA receptor subunit gene expression in the rat hippocampus caused by increased level of interleukin-1 $\beta$ in early postnatal ontogenesis // 17th International Pushchino School Conference of Young Scientists "Biology - The Science of the XXI Century", Pushchino, Russia, 22-26.04.2013 (oral presentation)

24. Veniaminova EA, Zubareva OE. The influence of interleukin-1 $\beta$ injections in early postnatal ontogenesis on mRNA expression of NMDA-receptor subunits in rat hippocampus. The XLII theoretical and practical conference with international participation «The week of science and academic research at SPBSPU», St. Petersburg, Russia, 1-6.12.2012 (oral presentation) 


\section{List of publications}

1. Veniaminova E, Oplatchikova M, Bettendorff L, Kotenkova E, Lysko A, Vasilevskaya E, Kalueff A, Fedulova L, Umriukhin A, Lesch KP, Anthony DC, Strekalova T. Prefrontal cortex inflammation and liver pathologies accompany cognitive and motor deficits following Western diet consumption in non-obese female mice. Life Sciences. 2020; 241:117163.

2. de Munter J, Shafarevich I, Liundup A, Pavlov D, Wolters E, Gorlova A, Veniaminova E, Umrukhin A, Kalueff A, Svistunov A, Kramer BW, Lesch KP, Strekalova T. Neuro-Cell therapy improves motor outcomes and suppresses inflammation during experimental syndrome of amyotrophic lateral sclerosis in mice. CNS Neurosci Ther. 2019; 00:1-14

3. Dukhinova M, Kuznetsova I, Kopeikina E, Veniaminova E, Yung AWY, Veremeyko T, Levchuk K, Barteneva NS, Wing-Ho KK, Yung WH, Liu JYH, Rudd J, Yau SSY, Anthony DC, Strekalova T, Ponomarev ED. Platelets mediate protective neuroinflammation and promote neuronal plasticity at the site of neuronal injury. Brain Behav Immun. 2018;74:7-27.

4. Pomytkin I, Costa-Nunes JP, Kasatkin V, Veniaminova E, Demchenko A, Lyundup A, Lesch KP, Ponomarev ED, Strekalova T. Insulin receptor in the brain: Mechanisms of activation and the 
role in the CNS pathology and treatment. CNS Neurosci Ther. 2018;24(9):763-774.

5. Amakhin DV, Malkin SL, Ergina JL, Kryukov KA, Veniaminova EA, Zubareva OE, Zaitsev AV. Alterations in Properties of Glutamatergic Transmission in the Temporal Cortex and Hippocampus Following Pilocarpine-Induced Acute Seizures in Wistar Rats. Front Cell Neurosci. 2017;11:264.

6. Veniaminova E, Cespuglio R, Cheung CW, Umriukhin A, Markova N, Shevtsova E, Lesch KP, Anthony DC, Strekalova T. Autism-Like Behaviours and Memory Deficits Result from a Western Diet in Mice. Neural Plast. 2017;2017:9498247.

7. Veniaminova E, Cespuglio R, Markova N, Mortimer N, Cheung CW, Steinbusch HW, Lesch KP, Strekalova T. Behavioral features of mice fed with a cholesterol-enriched diet: deficient novelty exploration and unaltered aggressive behavior. Transl. Neurosci. Clin. 2016;2(2):87-95.

8. Malkin SL, Amakhin DV, Veniaminova EA, Kim KKh, Zubareva OE, Magazanik LG, Zaitsev AV. Changes of AMPA receptor properties in the neocortex and hippocampus following pilocarpineinduced status epilepticus in rats. Neuroscience. 2016;327:146-55.

9. Strekalova T, Costa-Nunes JP, Veniaminova E, Kubatiev A, Lesch KP, Chekhonin VP, Evans MC, Steinbusch HW. Insulin receptor sensitizer, dicholine succinate, prevents both Toll-like 
receptor 4 (TLR4) upregulation and affective changes induced by a high-cholesterol diet in mice. J Affect Disord. 2016;196:109-16.

10. Veniaminova E, Strekalova T. High fat and cholesterol consumption as pathogenetic factor of depression: possible molecular mechanisms. Neurochemical journal. 2015, 2016;1:33-41

11. Veniaminova E, Zubareva O. Delayed changes in exploratory behavior and fgf2 gene expression in rat brain cells after neonatal lipopolysaccharide administration. Neurochemical journal. 2015, 32 (2) $153-160$

\section{Paper under revision}

Veniaminova E, Cespuglio R, Chernukha I, Schmitt-Boehrer AG, Morozov S, Kalueff AV, Kuznetsova O, Anthony DC, Lesch KP, Strekalova T. Metabolic, molecular and behavioural effects of Western diet in serotonin transporter-deficient mice: rescue by heterozygosity? Frontiers in Neuroscience.

\section{Papers in preparation}

1. Veniaminova E, Hebert J, Probert F, Radford-Smith D, Morozov S, Kalueff A, Cespuglio R, Schmitt-Boehrer AG, Lesch KP, Anthony DC, Strekalova T. In mice the absence of the serotonin transporter exacerbates the behavioural and metabolic phenotype induced by a Western diet. 
2. Mostert J, Arteaga G, Becerra N, Bierens M, Bloemendaal M, Bosch A, Cabana J, Fernàndez N, Koch E, Konstanti P, Grimstvedt Kvalvik L, Li L, Niemeyer L, van Rooij D, Schweren L, Shi Y, Veniaminova E, Willemse Y, Belzer C, Buitelaar J, Bullo M, Cormand B, Dietrich A, Ebner-Priemer U, Franke B, Haavik J, Haege A, Hartman C, Hoekstra P, Faraone S, Klunsyer K, Larsson H, Lesch KP, Ramos-Quiroga A, Reif A, Réthelyi J, Rommelse N, Rucklidge J, Strekalova T, Salas J, de Weerth C, Arias-Vasquez A. The role of diet, exercise and gut-microbiome in aggressive, impulsive and compulsive behaviour: the Eat2beNICE project. 